\title{
Ultrafast dynamics of the eumelanin pigment
}

\section{by Aleksandra Ilina}

\author{
Thesis \\ submitted to Victoria University of Wellington \\ in fulfillment of the requirements for the degree of \\ Doctor of Philosophy \\ in \\ Physics
}

Victoria University of Wellington

2022 



\section{Abstract}

Ultraviolet (UV) solar radiation can cause many hazardous effects in human body. It is highly carcinogenic; exposure to the sun radiation is leading to the malignant melanoma - the most aggressive and widely spread skin cancer. Most organic (including bioorganic) molecules can absorb the UV light which leads to the DNA photo-damage and the further mutations like tumour formation.

The black-brown pigment eumelanin found in human skin acts as a vital barrier to the UV light. With an ingenious ability to effectively dissipate $99.9 \%$ of the incoming UV energy as heat it has shown itself as a natural photo-protectant. The effective photoprotection of the pigment originates in its absorption spectrum. Unlike other organic molecules, eumelanin possesses a broadband featureless spectrum, rising in absorption coefficient toward the UV range, providing the increased photoprotection against the most damaging high energy photons. Eumelanin dissipates the absorbed energy on the picosecond timescale before the damage can occur. Therefore, the complexity of the pigment structure, as well as the ultrafast character of the energy dissipation complicate the characterization of the main energy dissipation pathway.

Over the last years, the pigment was extensively studied using different spectroscopic methods. To overcome the complexity of eumelanin's structure the main building blocks 5,6-dihydroxyindole (DHI) and 5,6-dihydroxyindole-2-carboxylic acid (DHICA) were studied. Ultrafast spectroscopy studies of eumelanin and its constituent building blocks have variously suggested the importance of excited state intramolecular proton transfer in oligomers, proton-coupled electron transfer with water, as well as charge transfer states. Significant inconsistencies between these 
studies raise questions about the validity of conclusions, and even whether the material measured was in the state assumed.

The bottom-up approach to eumelanin photophysics through its building block DHICA is reflected in Chapter 3 of this thesis. We used the combination of transient absorption and photoluminescence techniques to get a full picture of the excited state behaviour, as well as to track the possible formation of the new species due to degradation. The new way of preparing the samples in oxygen-free conditions revealed a new insight in the building block's photophysics. We then studied both proposed proton transfer mechanisms by placing DHICA monomer in different experimental environments, created by various solvents. We conclude that DHICA intrinsically decays on a 4-ps timescale via an excited-state proton transfer mechanism.

To investigate the role of oligomerization in the excited state decay rate we performed ultrafast spectroscopy studies on DHICA oligomers prepared in two different ways. Chapter 4 of the thesis explains the discrepancy that emerged between our results and the ones previously published. It was revealed that the presence of oxygen in results in oxygenated polymerization of the sample and the elongation of the excited state lifetime. This effect originated in the negative charge formation that created a barrier to proton transfer to occur. The study of the synthesized DHICA dimer demonstrated that its excited state decay is matching that of DHICA monomer. These studies revealed the importance of DHICA monomer, possessing ultrafast excited state proton transfer energy dissipation mechanism.

Chapter 5 of this thesis investigates the different hypotheses about the photophysics of eumelanin. By combining the computational and experimental methods we reveal the dynamic role of the disorder in eumelanin and the role of immobile charge transfer states that was proposed as a possible energy dissipation mechanism. Fluence and excitation dependent transient absorption spectroscopy revealed that the excitations in eumelanin are immobile and confined to a small volume. The comparison of the excited state decays of DHICA monomer and eumelanin revealed that these samples share the same decay rate. This suggests that eumelanin possesses the same excited state proton transfer mechanism as DHICA. 
Taken together this thesis provides a deep and valuable insight of eumelanin photophysics using time-resolved ultrafast spectroscopic techniques. The results of the studies of both eumelanin aggregates and DHICA key building block provide enough evidence to propose excited state proton transfer as the main excited state deactivation mechanism. 


\section{Acknowledgements}

A PhD is a journey. And it is never a solo journey. And while I cannot mention everyone who helped me, encouraged me, believed in me along the way, there are some people that I would especially like to thank.

First and foremost, I would like to express gratitude to my supervisor, Professor Justin Hodgkiss for his support, guidance and pushing me forward to improve myself as a researcher, especially through the troubling times of COVID-19 outbreak. Without his guidance I would never become who I am now.

I could not achieve anything without the constant support from my research group - my PhD peers Isabella, Clement, Sreelakshmi, Karen, Ronnie, Sai, and postdocs Dr Paul Hume, Dr Mike Price and Dr Kai Chen. Thank you for supporting me and advising me when I needed it the most and also for all the fun we have had throughout these years.

My friends, inside and outside of the University, in New Zealand and in Russia, who made my days brighter and better - Emily, Isabella, Sam, Shalini, Amira, Fraser, Anya, Rita, Sasha, Will, Ani, Harry, Zeineb, Nick, Nate, Andrew, Ethan, Kelly, Lucy and many, many others! Special gratitude goes to Dr Nate Davis and his group for the multiple equipment loans and valuable advises, A. Prof. Joanne Harvey and her group to assist me with the high vacuum training, and Sarah Adreassend for her immense help with HPLC and NMR experiments.

While I can continue thanking multiple people I met in these years, some of them deserve a special gratitude. Isabella Wagner, being an almost non-stop support throughout my PhD years, always happy to help with PL experiments, crucial for this research. Emily Jones, one of my best friends, not only helping me deal with the 
research anxiety, but also providing helpful feedback after proofreading this entire thesis. Dr Paul Hume, being a massive help with brainstorming through the trickiest parts of the eumelanin's photophysics. And last, but not least, Dr Karen Thorn for proofreading some of the chapters and sharing the eumelanin anxiety with me.

From the bottom of my heart I am grateful to my family - my mom Elena and my brother Andrey. They are always on my side, no matter what, supporting me from across the globe, and without them I would not be where I am today. Their continuous encouragement gave me the energy to keep going even when I was at the low points of my enthusiasm.

Last but not least, I thank Marsden Fund (and later, the MacDiarmid Institute for post-COVID support) for funding my scholarship, and Victoria University of Wellington for providing a supportive and friendly environment for my studies. 


\section{Table of Contents}

List of Figures $\ldots \ldots \ldots \ldots \ldots \ldots \ldots \ldots \ldots \ldots \ldots \ldots \ldots \ldots \ldots$

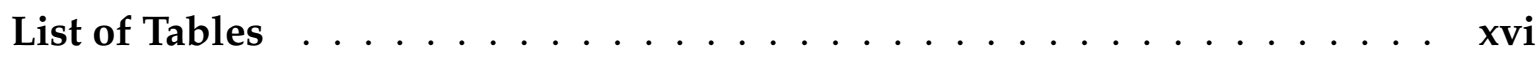

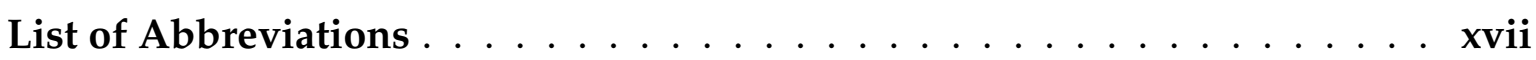

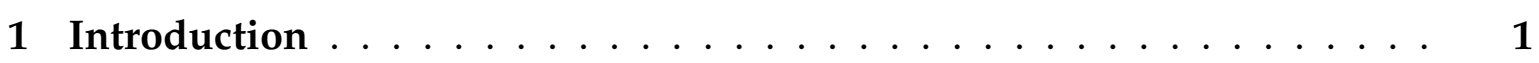

1.1 Chemical and molecular structure . . . . . . . . . . . . . 4

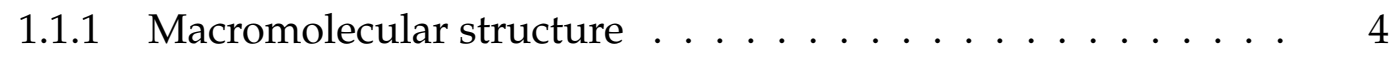

1.1 .2 Synthetic eumelanin $\ldots \ldots \ldots \ldots \ldots \ldots \ldots$

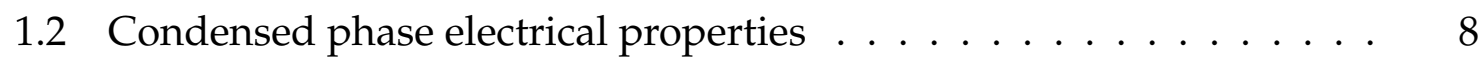

1.2.1 Amorphous semiconductor model . . . . . . . . . . . 8

1.2.2 Charge transport in eumelanin . . . . . . . . . . . . 12

1.3 Optical properties . . . . . . . . . . . . . . . . . 13

1.3.1 Broadband absorption spectrum . . . . . . . . . 13

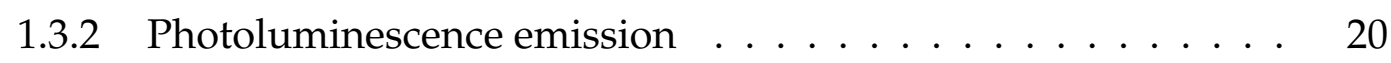

1.4 Photoprotective properties . . . . . . . . . . . . . . 21

1.5 Ultrafast nonradiative relaxation dynamics $\ldots \ldots \ldots \ldots$

1.5.1 Excitation energy transfer between the chromophores . . . . 25

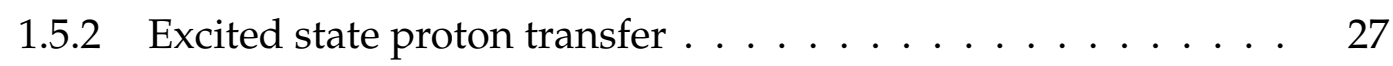

1.5.3 Charge transfer states recombination . . . . . . . . . 30

2 Experimental approach and $\operatorname{methods} \ldots \ldots \ldots \ldots$

2.1 Ultrafast optical spectroscopy $\ldots \ldots \ldots \ldots$

2.2 Examples of the generated femtosecond pulses . . . . . . . . 36 
2.2.1 Nonlinear optics . . . . . . . . . . . . . . . . . . . 36

2.2.2 Second harmonics generation . . . . . . . . . . . . 37

2.2.3 Third harmonics generation setup build to expand the current setup . . . . . . . . . . . . . . . . . . 39

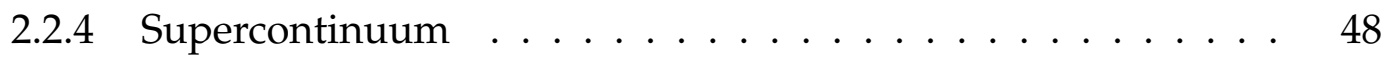

2.2.5 Optical parametric amplification . . . . . . . . . . 50

2.3 Transient absorption spectroscopy $\ldots \ldots \ldots \ldots \ldots$

2.4 Transient grating photoluminescence spectroscopy . . . . . . . . . 58

3 Ultrafast spectroscopy of the main eumelanin building blocks DHICA

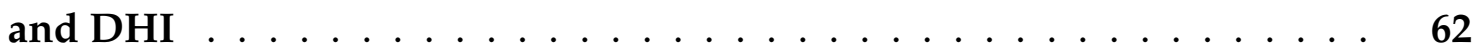

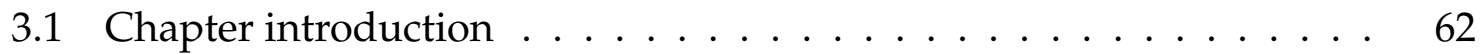

3.1.1 Photophysics of DHICA key building block . . . . . . . . . 62

3.1.2 Photophysics of DHI key building block . . . . . . . . . . 67

3.2 Ultrafast spectroscopy results of DHICA monomer . . . . . . . . . 68

3.2.1 Sample preparation . . . . . . . . . . . . . . . . . . 69

3.2.2 DHICA as photoacid . . . . . . . . . . . . . 72

3.2.3 The effect of the solvent environment on DHICA excited-state

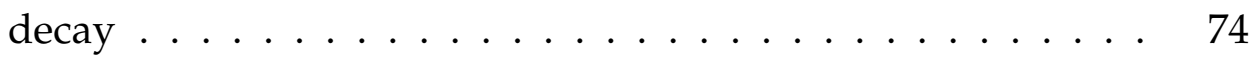

3.2.4 Radical formation in alkaline conditions eliminates the likely role of PCET . . . . . . . . . . . . . . . . . 80

3.2.5 Role of the oxygen in the spectroscopic measurements . . . . 86

3.3 Ultrafast spectroscopy results of DHI . . . . . . . . . . . . . . 93

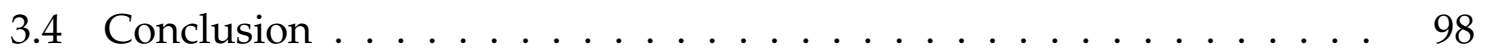

4 Role of oligomerization in DHICA energy dissipation . . . . . . . . 100

4.1 Chapter introduction . . . . . . . . . . . . . . . . 100

4.2 Sample preparation and steady-state characterisation . . . . . . . 103

4.3 Experimental results . . . . . . . . . . . . . . . . . . . 105

4.3.1 Transient absorption spectroscopy of DHICA dimer . . . . . 105

4.3.2 Effect of the uncontrolled polymerization on the DHICA excitedstate decay . . . . . . . . . . . . . . . . . 110 
4.4 Conclusion . . . . . . . . . . . . . . . . . . . . . . . . . 114

5 Ultrafast spectroscopy of eumelanin pigment . . . . . . . . . . . . 117

5.1 Chapter introduction . . . . . . . . . . . . . . 117

5.1 .1 Sample preparation . . . . . . . . . . . . 120

5.2 Role of chemical disorder in the energy dissipation of eumelanin . . . 120

5.2.1 Matlab-based simulation for energy transfer in multichromophore system ......................... 123

5.2.2 Transient absorption results reveal localized excitations . . . . 132

5.2.3 Charge transfer states recombination . . . . . . . . . . . . 135

5.3 Proton transfer mechanism in eumelanin . . . . . . . . . . . . 140

5.4 Conclusion . . . . . . . . . . . . . . . . . 145

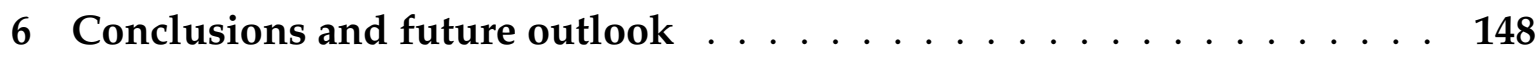

A Matlab code used for energy transfer simulations in multichromophore system ........................ 154 


\section{List of Figures}

Figure 1.1 - Estimated age-standardized incidence and mortality rates in 2020 from both melanoma and non-melanoma skin cancer . . . . 2

Figure 1.2 - Broadband absorption of synthetic eumelanin. . . . . . . . 3

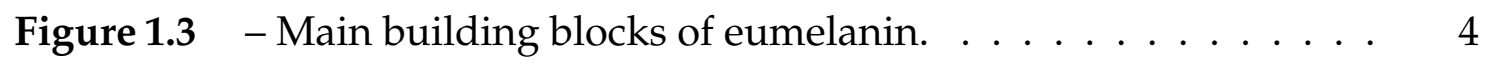

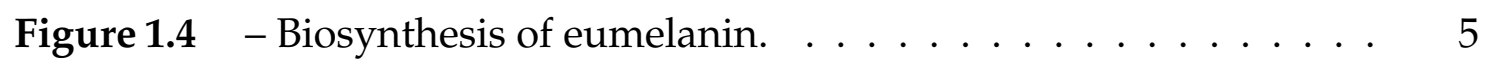

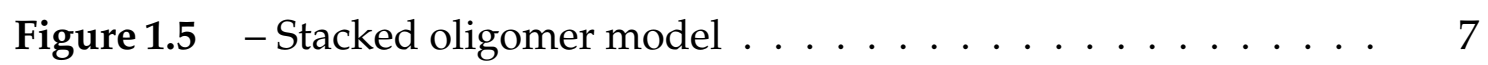

Figure 1.6 - Eumelanin conductivity as a function of water content for (a) sandwich contact geometry and (b) van der Pauw contact geometry. 10

Figure 1.7 - Melanin photoconductivity for two different hydration states. 11

Figure 1.8 - The scattering coefficient as a percentage of the total optical

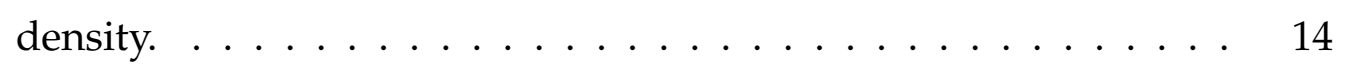

Figure 1.9 - DHI redox forms (adapted from [138]). . . . . . . . . . 15

Figure 1.10 - Equivalent DHICA redox forms (adapted from [137]). . . . 16

Figure 1.11 - Simulation of eumelanin broadband absorption spectrum using a linear combination of 11 Gaussians. Reproduced using the approach from [111]. The exponential envelope is shifted for clarity. 17

Figure 1.12 - Absorption spectrum of eumelanin as a result of the superposition of the dominant tetramers spectrum (thick black line, shifted up by 1.5 units for clarity). Tetramers' spectra are shown by the thin coloured lines, monomers' spectra are shown by the black lines (shifted down by 1 unit for clarity). Reprinted with permission from [87]. Copyright (2006) by the American Physical Society. 18

Figure 1.13 - Excitation spectra showing wavelength-dependent emission. Reprinted from [123], with the permission of AIP Publishing. . . . 20 
Figure 1.14 - Degenerate $350 \mathrm{~nm}$ pump-probe transient absorption data. Reprinted with permission from [126]. Copyright (C) 2001, American Chemical Society. . . . . . . . . . . . . . . . . . . .

Figure 1.15 - Fluorescence up-conversion decays of DHICA dimer in water and polyvinyl alcohol buffer and DHICA polymer (PM) in polyvinyl alcohol buffer. Reprinted with permission from [37]. Copyright (C) 2014, American Chemical Society. . . . . . . . . . . . . . . 24

Figure 1.16 - EET process between two chromophores. Adapted from [105]. 26

Figure 1.17 - Energy migration by excited energy transfer. Adapted from

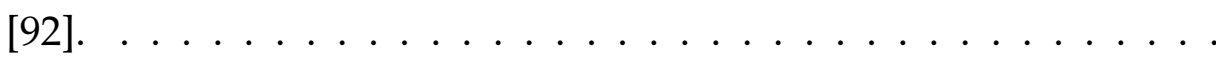

Figure 1.18 - Excited state proton transfer. The pump induces the $A H$ molecule to its excited state $(A H)^{*}$ leading the an electronic redistribution which results in a proton transfer from $(A H)$ * to $B$. Adapted from $[1] \ldots \ldots \ldots \ldots \ldots \ldots \ldots \ldots$

Figure 1.19 - Scaled absorption (blue) and emission (red) spectra of DHICA in aqueous solution. Reprinted with permission from [128]. Copyright (C) 2007, American Chemical Society. . . . . . . . . . . . .

Figure 1.20 - Fluorescence decay of DHICA at pH 3, pH 7 and in acetonitrile. Reprinted with permission from [128]. Copyright (C) 2007, American Chemical Society. . . . . . . . . . . . . .

Figure 1.21 - Summary of the ultrafast excited state deactivation of the DHICA oligomer via excited-state proton transfer processes. Reprinted with permission from [37]. Copyright (C 2014, American Chemical Society. . . . . . . . . . . . . . . . 31

Figure 1.22 - Ground state bleach dynamics from possible excited-state decay pathways. The colors of the peaks represent the different absorption energies - from high (violet) to low (red). Adapted from [92]

Figure 1.23 - Polarization-dependent spectra of the synthetic eumelanin. Reproduced from [92] with permission from the Royal Society of

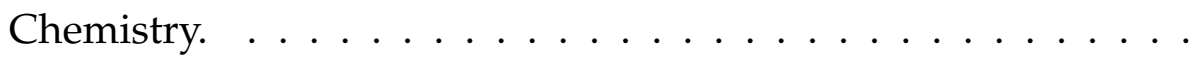


Figure 2.1 - Geometry of the second harmonic generation and the energy level diagram of the process. Adapted from [17]. . . . . . . . . . 38

Figure 2.2 - Third harmonic generation process. . . . . . . . . . . 41

Figure 2.3 - Two-step process of THG. The time delays from the crystals are not indicated. . . . . . . . . . . . . . 41

Figure 2.4 - Third harmonics generation setup. . . . . . . . . . . 42

Figure 2.5 - Different paths for ordinary (AC) and extraordinary (AB) waves in the birefringent crystal. . . . . . . . . . . . . 44

Figure 2.6 - Tuning curve for a BBO compensation plate. . . . . . . . . 46

Figure 2.7 - Third harmonics signal from the built setup. . . . . . . . . . 48

Figure 2.8 - White light continuum, produced by $\mathrm{CaF}_{2}$ and YAG crystals. The drop of intensity around $800-850 \mathrm{~nm}$ region is coming form blocking the $800 \mathrm{~nm}$ contribution and merging visible and IR cameras at $850 \mathrm{~nm} \ldots \ldots \ldots 50$

Figure 2.9 - Layout for a transient absorption system. . . . . . . . . . 52

Figure 2.10 - Example of TA data for eumelanin: surface (a), spectral traces

(b) and kinetics traces (c) . . . . . . . . . . . . . . 55

Figure 2.11 - The types of signals, contributing in the TA spectra. Adapted from [152]. . . . . . . . . . . . . . . . . . 56

Figure 2.12 - Example of the chirp correction in the TA data of the neat Y6 material: TA surface before (a) and after (b) chirp correction. . . . 57

Figure 2.13 - Schematic depiction of the broadband TGPL method. . . . . 59

Figure 2.14 - Example of TGPL data of eumelanin: surface (a), spectral traces $(b)$ and kinetics traces (c) . . . . . . . . . . . . . 60

Figure 3.1 - Mirror-image rule violation in DHICA monomer. . . . . . 63

Figure 3.2 - Excited-state intramolecular proton transfer in DHICA monomers in acidic environment. . . . . . . . . . . . . . . . . 65

Figure 3.3 - Proposed excited-state proton transfer to the solvent in DHICA monomers in neutral environment. . . . . . . . . . . 66

Figure 3.4 - Studied forms of DHICA monomer in the solvents of differ-

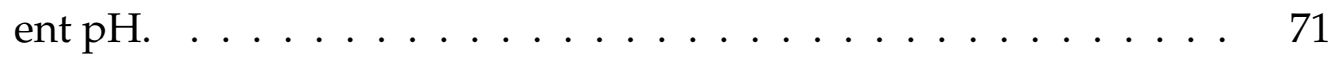


Figure 3.5 - Steady-state absorption (solid lines) and emission (dashed lines) spectra of the DHICA monomer in different solvents. . . . . 72

Figure 3.6 - Proton transfer process in photoacids (the Förster cycle) in neutral conditions. . . . . . . . . . . . . . . . 73

Figure 3.7 - Break-down of the 4-level system into parts to study each of them. ...................... 76

Figure 3.8 - Transient absorption spectra (a) and kinetics (b) of DHICA monomer in degassed water. . . . . . . . . . . . . 77

Figure 3.9-Comparison of the TA and PL decay of DHICA monomer in degassed water. . . . . . . . . . . . . .

Figure 3.10 - Comparison of of the transient absorption signal decays for the neutral DHICA and carboxylate monoanion. . . . . . . . 78

Figure 3.11 - Comparison of TRPL results for different forms of DHICA. 79

Figure 3.12 - Schematic depiction of the proposed excited state deactivation via ESPT (on the left). The right panel proposes the depiction of the ESPT process in DHICA monomer for potential energy surfaces. . . . . . . . . . . . . . . .

Figure 3.13 - Comparison of TRPL spectra (a) and kinetics (b) of DHICA in neutral and alkaline conditions. . . . . . . . . . . . . . . . . 81

Figure 3.14 - Time integrated intensity-dependent steady-state emission. $\quad 82$

Figure 3.15 - Fluence-dependent TRPL spectra, normalised to the first peak's intensity (a) and kinetics of the first peak (b). . . . . . . . . 83

Figure 3.16 - Nonlinear power dependence of the second PL peak. . . . . 84

Figure 3.17 - Proposed potential energy surfaces of the formation of the dianion radical. . . . . . . . . . . . . . . . . . . .

Figure 3.18 - TRPL surface of DHICA in basic water, excited at the highest fluence $86.81 \mu \mathrm{J} / \mathrm{cm}^{2}$ : full surface (a), and first 20 ps after excitation

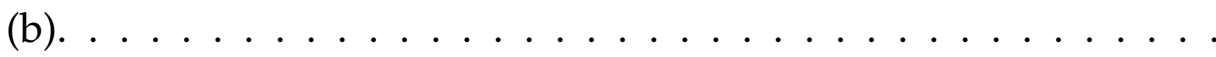

Figure 3.19 - Scheme of the proposed photoproduct formation in alkaline conditions and the comparison of the PCET and ESPT processes in neutral conditions. . . . . . . . . . . . . . . . . . 
Figure 3.20 - Absorption of DHICA monomer as it undergoes UV-induced oxidative polymerization in aerated water. The dashed curve shows the final absorption spectrum after $72 \mathrm{~h} . \ldots \ldots \ldots$

Figure 3.21 - The changes in the steady-state absorption of DHICA monomer in the presence of oxygen in the dark. . . . . . . . . . . .

Figure 3.22 - Evolution of transient absorption spectra of DHICA in aerated water. . . . . . . . . . . . . . . . . 89

Figure 3.23 - MCR-ALS results of DHICA in aerated water. . . . . . . . 90

Figure 3.24 - TRPL spectra (a) and kinetics (b) of DHICA in aerated water. 90

Figure 3.25 - TA spectra of DHICA that went thought the oxidative polymerization (a); comparison of the spectral shapes of DHICA at the different stages of polymerization $(b) \ldots \ldots . \ldots 91$

Figure 3.26 - TA (a) and TRPL (b) kinetics of DHICA in degassed and aer-

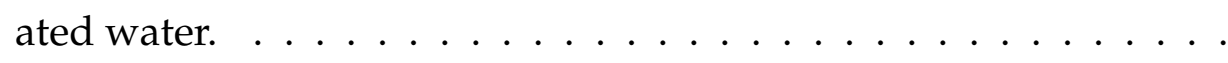

Figure 3.27 - The comparison of UV-Vis of the newly made aged DHI sample a month after the first TA measurement (red) with the UVVis spectrum of the fresh DHI before the first TA measurement (black). . . . . . . . . . . . . . . . 95

Figure 3.28 - Transient absorption spectra of the fresh DHI sample. . . . 96

Figure 3.29 -Comparison of transient absorption bands of the aged (dashed) and the fresh (solid) DHI sample. . . . . . . . . . . . . 97

Figure 4.1 - The proposed pathways for the ESPT in 4,4'-DHICA dimer. 102

Figure 4.2 - Steady-state absorption for two DHICA samples dissolved in phosphate buffers of different pH. . . . . . . . . . . . . . . 104

Figure $4.3 \quad-{ }^{1} \mathrm{H}-\mathrm{NMR}$ spectrum of 4,4'-dimer in DMSO-d6. . . . . . . . . 104

Figure 4.4 - Steady-state absorption of DHICA monomer and dimer. . . 105

Figure 4.5 - TA spectra of DHICA dimer in water. . . . . . . . . . . 106

Figure 4.6 - Comparison for TA spectra (a) and kinetics (b) of DHICA monomer and dimer. . . . . . . . . . . . . . . . 107

Figure 4.7 - Comparison of TA spectra (a) and kinetics (b) of DHICA monomer and dimer, obtained in aerated conditions . . . . . . . 108 
Figure 4.8 - Spectral traces for DHICA in aerated pH 3 buffer (a) and aerated water (b), presented in Chapter 3, Section 3.2.5, Figure 3.22; comparison of their early-time and later-times spectra (c) and excited state kinetics for the two oligomeric forms of DHICA with monomer $(\mathrm{d}) \ldots \ldots \ldots \ldots \ldots$

Figure 4.9 - Comparison of the decays of different forms of DHICA, depending on the polymerization and measurement conditions. The obtained results were compared with the decay of DHICA monomer in degassed water. . . . . . . . . . . . . . . . . . 11

Figure 5.1 -Steady-state absorption of eumelanin film. . . . . . . . . 121

Figure 5.2 - Transient absorption spectra of the thin films of synthetic eumelanin, excited at different wavelengths across the spectral range. The shaded area hides the lack of the spectra due to the weak supercontinuum signal. . . . . . . . . . . . . .

Figure 5.3 - Different scenarios of the excitation in the multichromopore system. (a) Excitation energy transfer between the chromophores. The colors are showing the energy decreasing from high (violet) to the low (red) energy; (b) spectral representation of the excitation energy transfer. (c) Excitation of the distribution of chromophores, each having a residual absorption peak in UV and (d) its spectral representation. . . . . . . . . . . . . . .

Figure 5.4 - Simulated surface (a), consistent of spectra (b) and kinetics (c).127

Figure 5.5 - The result of the simulated behaviour of the multicomponent system. The presented surface (a) is consistent of the simulated spectra (b) and kinetics (c). . . . . . . . . . . . . .

Figure 5.6 - Spectral masks translated along the energy axis five times with the $0.2 \mathrm{eV}$ spacing as shown in the top panel. The result shows an agreement with the real data at $400 \mathrm{~nm}$ excitation. The form of the spectral mask was taken from the spectral shape at $712 \mathrm{~nm}$ excitation. . . . . . . . . . . . . . . 130 
Figure 5.7 - Photoluminescence surface for synthetic eumelanin thin film excited at $267 \mathrm{~nm}$ : full surface at $90 \mathrm{ps}$ after excitation (a) and first 10 ps after excitation $(b) . \ldots \ldots \ldots$. . . . . . . . . .

Figure 5.8 - Fluence independence for the kinetics traces of eumelanin thin film at $400 \mathrm{~nm}$ excitation (a). Singlet-singlet exciton annihilation in neat IDIC film (b). The figure (b) is reprinted with permission from [28]. Copyright 2019 American Chemical Society. . . . .

Figure 5.9 - Previously published polarization-dependent spectra of the synthetic eumelanin. Reproduced from [92] with permission from the Royal Society of Chemistry (a). Compared with the results of polarization-dependent TA spectra at $250 \mathrm{fs}$ for DHICA monomer (b) and eumelanin film $(\mathrm{c}) . \ldots \ldots \ldots$

Figure 5.10 - The change in anisotropy for PIA, calculated for DHICA monomer (a) and eumelanin (b) . . . . . . . . . . . . . . . 138

Figure 5.11 - Photoluminescence polarization anisotropy of DHICA monomer

(a) and synthetic eumelanin film (b). . . . . . . . . . . . . . 139

Figure 5.12 - Comparison of the TA spectra of eumelanin and DHICA monomer in degassed water. . . . . . . . . . . . . . . . 141

Figure 5.13 - TA (a) and TRPL (b) excited state kinetics of DHICA monomer and eumelanin film. . . . . . . . . . . . . . . . . . . 142

Figure 5.14 - TRPL spectra of eumelanin film, excited at $267 \mathrm{~nm}$ (top panel) and $343 \mathrm{~nm}$ (bottom panel). . . . . . . . . . . . . . . . 143

Figure 5.15 - Vibrational spectra for eumelanin and DHICA monomer (a); Individual spectral slices (b). The data is collected and presented by Dr. Karen Thorn of Victoria University of Wellington and reprinted with permission from [170] with the provisions of the Copyright Act 1994 (New Zealand). . . . . . . . . . . . . . . . . . . 145

Figure 6.1 - The proposed oligomeric structure, containing both DHI and DHICA in different redox forms. . . . . . . . . . . . . . . . 152

Figure 6.2 - The proposed incorporation of DHICA quinones to study the oxidative polymerization. . . . . . . . . . . . . 153 


\section{List of Tables}

Table 2.1 - List of crystals for the third harmonic generation. . . . . . . . 47

Table 4.1 - Lifetimes for the different DHICA polymerization and measurement conditions. . . . . . . . . . . . . . . . . . . . 114 


\section{List of Abbreviations}

BBO beta barium borate

$\mathrm{CaF}_{2}$ calcium fluoride;

CT charge transfer

DHI 5,6-dihydroxyindole

DHICA 5,6-dihydroxyindole-2-carboxylic acid

DMSO-d6 dimethyl sulfoxide-d6

DNA deoxyribonucleic acid

EET excitation energy transfer

ESIPT excited state intramolecular proton transfer

ESPT excited state proton transfer

GSB ground state bleach

HHI 6-hydroxy-4-dihydro-indole-5-one

HOMO highest occupied molecular orbital

IR infrared

IRF instrument response function 
KDP potassium dihydrogen phosphate

LUMO lowest unoccupied molecular orbital

MCR-ALS Multivariate Curve Resolution-Alternating Least Squares

MDAS Mott-Davis amorphous semiconductor

$\mathrm{NaOH}$ sodium hydroxide

$\mathbf{N H}_{3}$ ammonia

NIR near-infrared

NMR nuclear magnetic resonance

OPA optical parametric amplifier

PCET proton-coupled electron transfer

PIA photoinduced absorption

PL photoluminescence

SHG second harmonics generation

TA transient absorption

TGPL transient grating photoluminescence

THG third harmonics generation

UV ultraviolet

YAG yttrium-aluminium garnet 
Chapter 1

\section{Introduction}

Ultraviolet (UV) solar radiation that reaches the Earth surface is the major factor responsible for many hazardous effects on the human skin. Those effects include erythema (sunburn), immunosuppression, photo-aging (wrinkles) and the breakage of the DNA strand that leads to tumour formations [20]. UV radiation is responsible for the formation of various skin cancers such as basal skin carcinoma, squamous cell carcinoma and malignant melanoma [113].

Skin cancer is one of the most common cancers in the world [88]. In 2018 nonmelanoma skin cancer was the 5th most commonly occurring cancer in both men and women while melanoma of the skin was the 19th most commonly occurring cancer in both men and women [19]. Malignant melanoma, while being the least common of the skin cancers, has $60 \%$ mortality rate worldwide. The incidence of melanoma and non-melanoma skin cancers has increased throughout the world, with the most cases registered in Australia and New Zealand [64]. The statistics of the melanoma and non-melanoma skin cancers incidence and mortality in 2020 is shown in Figure 1.1.

The skin's response to the UV irradiation is pigmentation (tanning). After being exposed to the UV light the skin starts to produce the pigment called eumelanin the most common black-brown pigment of the class of melanins [173]. Melanins are produced through a mechanism called melanogenesis, the oxidation of the amino acid tyrosine and the following polymerization [142]. There are three main types of melanin: eumelanin, pheomelanin, and neuromelanin. Together with the redyellow pigment pheomelanin, eumelanin is usually found in the skin, hair and eyes of humans and many different animal species [76]. Neuromelanin is found in the human brain and central nervous system. It was discovered that the individuals with lesser neuromelanin content (or its absence) have a greater risk for developing Parkinson's disease [52, 54]. 


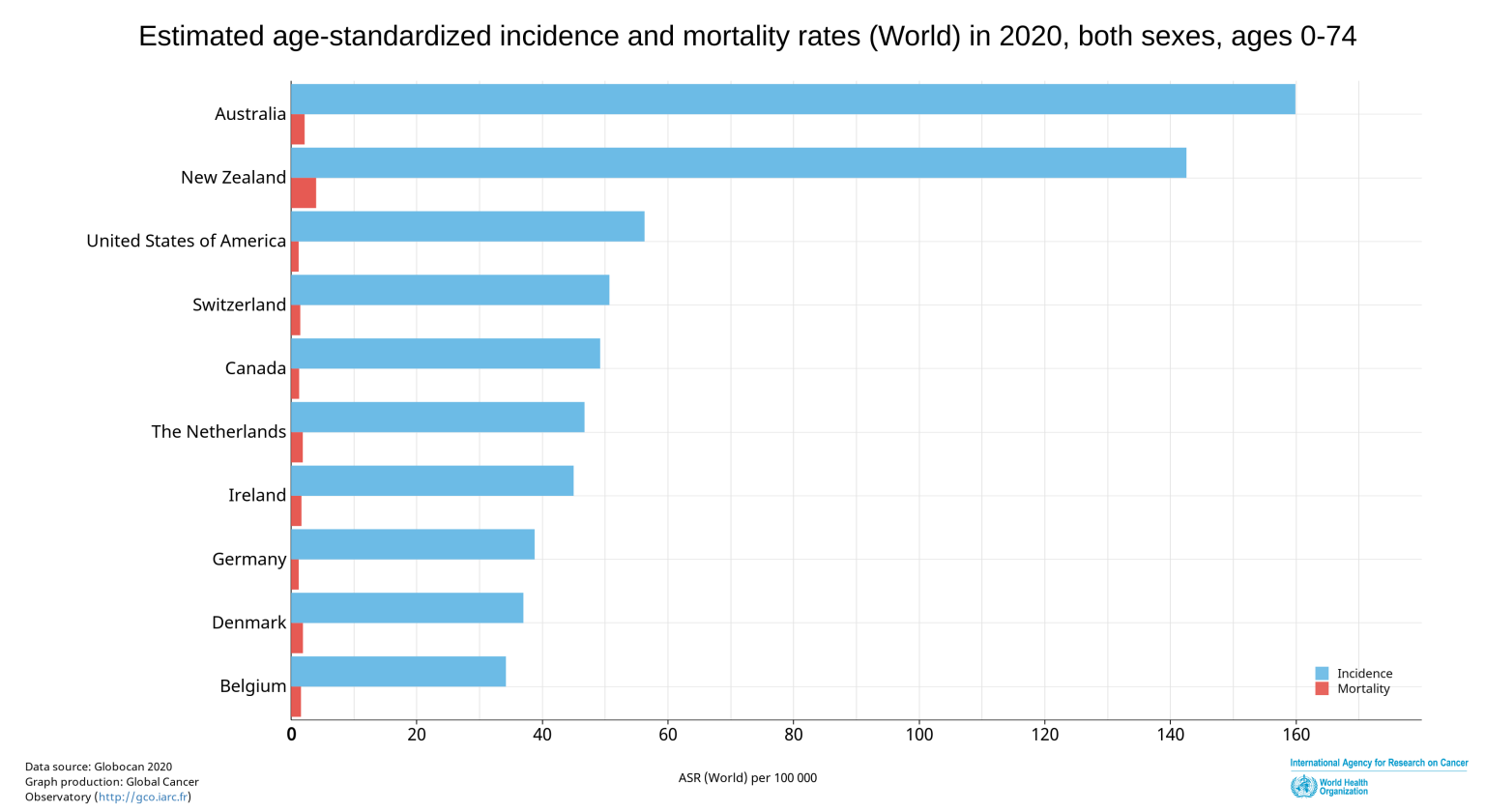

Figure 1.1: Estimated age-standardized incidence and mortality rates in 2020 from both melanoma and non-melanoma skin cancer. Reprinted with from IARC (C) International Agency for Research on Cancer [64].

Eumelanin is the most extensively studied of all the melanins because it is a predominant pigment in the human skin [176] and therefore has a primary role in humans pigmentation. The main interest in eumelanin was attracted by its ingenious ability to effectively dissipate $99.9 \%$ of harmful solar UV-radiation to heat [113]. That makes eumelanin a very effective natural photoprotectant in human skin. The photoprotection nature of the pigment originates in its absorption spectrum (Figure 1.2) - broad, rising from NIR towards UV it has no gaps allowing the harmful irradiation to get to the body [113]. The absorbed energy is dissipated within a few picoseconds before damage can occur to underlying cells [74].

Despite its importance, little is known about the dissipation mechanism responsible for this effective thermal dissipation. The lack of knowledge about such an effective excited state deactivation originates in eumelanin complexity and the strong disorder of the system. Over the last two decades the excited state deactivation remained the subject of an intensive study [37, 38, 60, 61, 73, 110, 111, 126, 183]. Later 


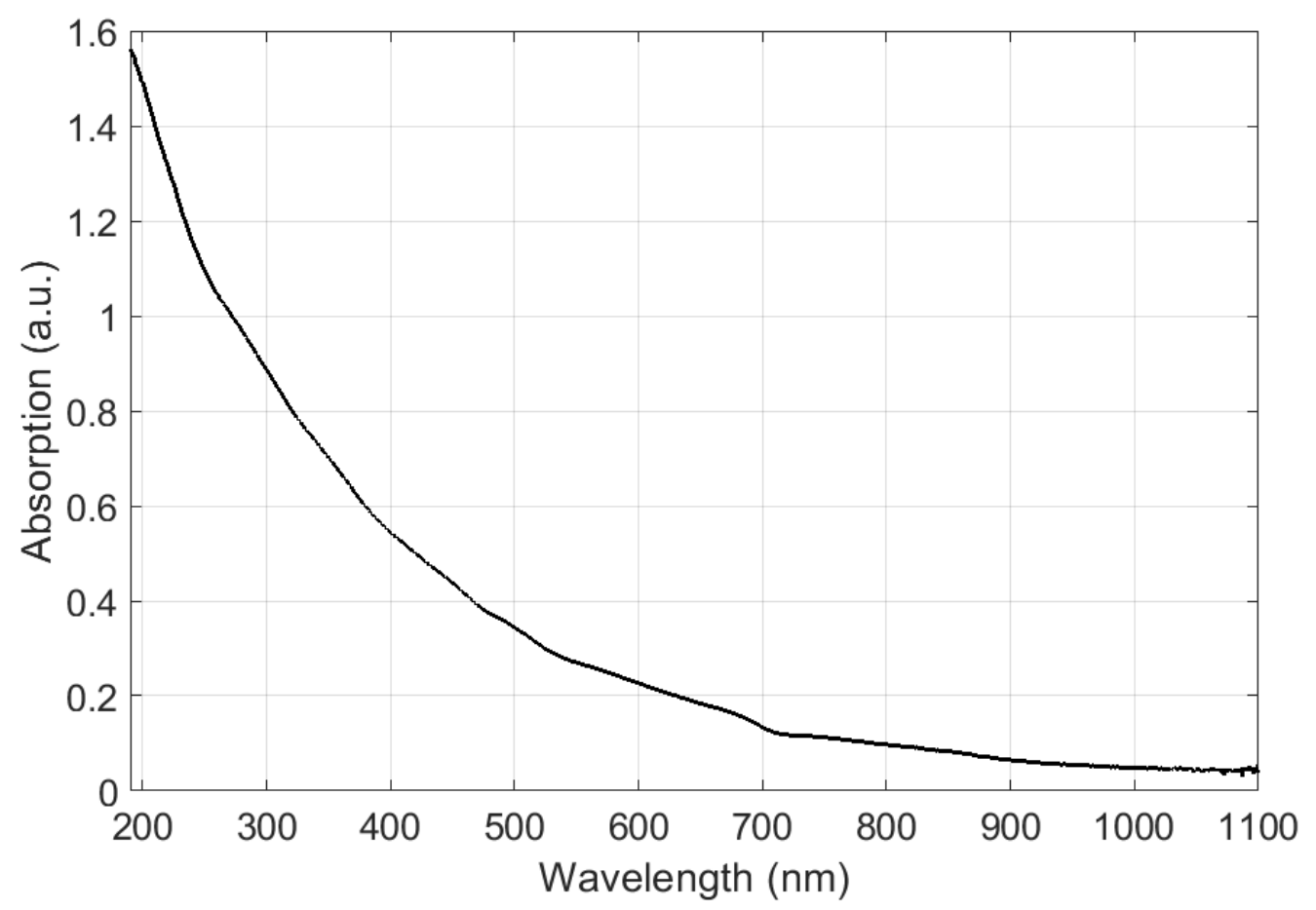

Figure 1.2: Broadband absorption of synthetic eumelanin. 
in this chapter we will advert to these theories.

\subsection{Chemical and molecular structure}

\subsubsection{Macromolecular structure}

In human cells, melanin is packed in cytoplasmatic organelles called melanosomes which can vary in shape and size. The structure of those melanosomes is not definite and strongly depends on its location in the body and on the protein to which eumelanin is bonded [153].

At a primary level, eumelanin is known to be a macromolecule of two indole monomers: 5,6-dihydroxyindole (DHI) and 5,6-dihydroxyindole-2-carboxylic acid (DHICA) (Figure 1.3) and the variety of their redox forms. The building blocks are biologically derived from tyrosine oxidized by tyrosinase into dopaquinone [144, 145] as shown in Figure 1.4. The high instability of the reaction intermediates and insolubility of the final product in most solvents make the identification of the next steps of the biosynthesis nearly impossible [77].<smiles>Oc1cc2cc[nH]c2cc1O</smiles>

DHI<smiles>O=C(O)c1cc2cc(O)c(O)cc2[nH]1</smiles>

DHICA

Figure 1.3: Main building blocks of eumelanin.

DHICA and DHI building blocks can bond in many different ways and form oligomers of diverse shapes and lengths. The dimers and higher oligomers can be 


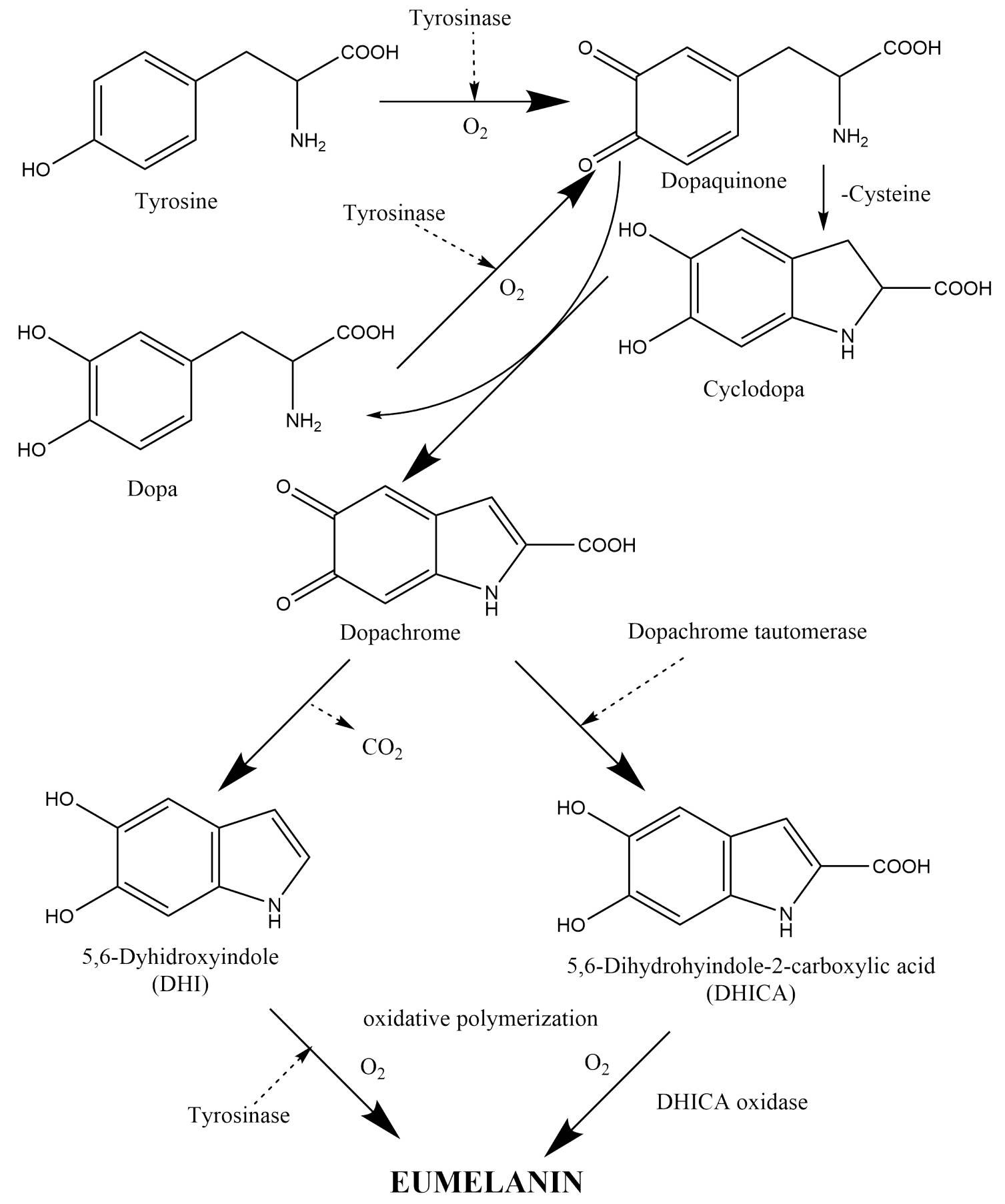

Figure 1.4: Biosynthesis of eumelanin. Adapted from [77]. 
produced in many various ways which leads to an extreme chemical disorder [111]. This is why, albeit many experiments have been conducted in chemistry, photophysics and biology fields [113] to understand the structure of eumelanin, there is no agreement on its secondary structure. It should be noted that here we use the nomenclature borrowed from proteins to refer to the "secondary structure" as to the macromolecular assembly of the monomers and formed oligomers.

Historically eumelanin was believed to be a highly conjugated heteropolymer $[101,143]$. This model would well describe some of the optical and electrical properties of eumelanin such as the broadband shape of the absorbance spectrum (Figure 1.2) and electrical and photo-conductivity [113], suggesting that eumelanin is an organic amorphous semiconductor (which will be described in Section 1.2). However, there are a wealth of evidence $[33,40,113,174]$ that the heteropolymeric model might not be the right way to describe the eumelanin system.

Several authors [36, 172, 174, 184], have been developing an alternative model to describe the pigment structure. They speculate that DHI and DHICA connect through $\pi$-stacking to form small (4-8 monomeric units [148]), flat oligomers which can assemble to form nanoaggregates called protomolecules as shown in Figure 1.5. This suggestion was made based mainly on X-ray scattering studies [32, 33]. In 2009, using high resolution transmission electron microscopy Watt et al. [174] showed that the synthetic eumelanin (that will be briefly discussed in the next section) protomolecules are consistent with the curved sheets of indolic subunits (2-10 nm or 5-10 subunits) that arrange in concentric rings to form the spherical onion-like nanostructures which was the image of the eumelanin protomolecule and an evidence for the stacking oligomer model.

For the natural eumelanin studies, the authors used bovine retinal epithelium eumelanin. The transmission electron microscopy of the pigment showed the similar highly stacked planar nanostructures but not packed into the spherical rings, like in the case of synthetic eumelanin. To confirm their finding, the eumelanin granules were extracted from the ink sac of the cuttlefish Sepia officinalis. In that case, the enzymatic or basic treating could be avoided and the scanning electron microscopy showed the small spherical structures. The stacked planes were once again seen in 

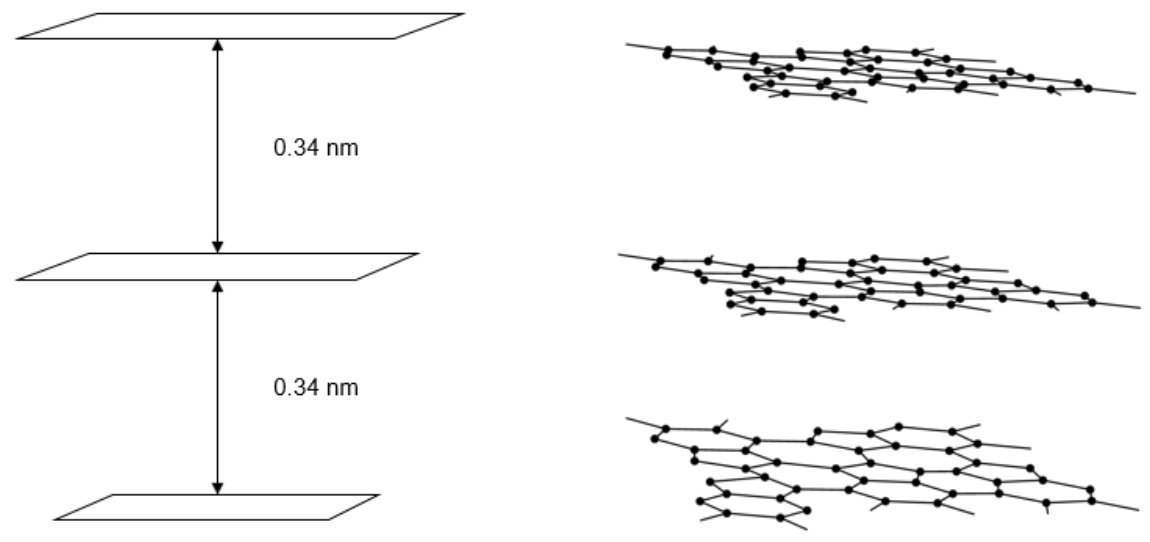

Figure 1.5: Stacked oligomer model - flat oligomers are stacked in z-plane. Adapted from [184].

the structure with the transmission electron microscopy image. This may serve as a confirmation that the structural image observed in synthetic eumelanin is repeated in the natural pigment and might be an intrinsic property of eumelanin. Larger polymeric structures have not been observed which may serve as evidence for the chemical disorder model which will be discussed later in this chapter.

\subsubsection{Synthetic eumelanin}

To study natural eumelanin, one must extract it from a biological source. It has been shown, however, that in the case of obtaining the pigment from the human hair, it requires harsh acid/base extraction to remove the bounded protein. Nevertheless, such procedures can't completely remove the protein without destroying the integrity of melanosome which will disrupt the properties of eumelanin [100]. It is now common to use eumelanin extracted from the ink sac of the cuttlefish Sepia officinalis as a model eumelanin pigment since it is easy to obtain and one can bypass the problem of removing the bounded protein [141].

Synthetic eumelanin is often used as a standard for studying natural eumelanin. It is commonly prepared by oxidation of tyrosine with hydrogen peroxide [93]. Syn- 
thetic eumelanin is a convenient model system to study the behaviour of the natural pigment since it is prepared under controlled conditions.

Despite the fact that the secondary structure remains unknown, the synthetic eumelanin shows optical and electrical properties similar to the natural pigment $[77,113]$. Although there is no proof $[142,166]$ that the synthetic compound is exactly identical to the natural one, it gives one a possibility to use it as a model for pioneering research of new methods and theories.

\subsection{Condensed phase electrical properties}

\subsubsection{Amorphous semiconductor model}

The suggestion that the broadband nature of the absorption spectrum of eumelanin (or, in other words, its black color) can be explained with its semiconducting properties came from the theoretical works by Longuet-Higgins in 1960 [101] and Pullman and Pullman in 1961 [143]. In this model, eumelanin consists of a large indole heteropolymer with a delocalized $\pi$ system that can explain the electron-acceptor and semiconducting properties.

The further investigation by McGinness [78, 108] suggested that the electronic properties of eumelanin can be explained with a Mott-Davis amorphous semiconductor (MDAS) model. This theory was supported by recovering the threshold switching behaviour in eumelanin pellets (which means they can switch between two resistive states with the application of a moderate electric field). Previously observed only in inorganic materials, switching behaviour is a defining feature of amorphous semiconductors. An interesting feature of these experiments was that when the pellets of eumelanin were dried they did not switch until re-hydrated. This can be explained with Rosenberg's theory that the dielectric constant of semiconducting organic materials can be modified by the presence of water $[139,150]$. For that, the Arrhenius equation for the temperature-dependent conductivity $\sigma(T)$ was modified: 


$$
\sigma(T)=\sigma_{0}(T) \exp \left(-\frac{E_{D}}{2 k T}\right) \exp \left[\frac{e^{2}}{2 k T R}\left(\frac{1}{\mathcal{\kappa}}-\frac{1}{\mathcal{\kappa}^{\prime}}\right)\right],
$$

where $\sigma$ is the conductivity, $E_{D}$ is the dry state activation energy, $\kappa$ is the dielectric constant in the dry state, $\kappa^{\prime}$ is the dielectric constant after hydration and $R$ is a constant dependent upon the effective screening radius of adsorbate. This modified MDAS model had support from the additional experiments [82, 139] showing the dependence of the conductivity on the sample hydration.

Mostert et al. [14] noticed that in these measurements the sandwich contact geometry was used. The previously reported water adsorption isotherms show that long timescales are required to achieve the equilibrium of water content in the eumelanin solid pellets [116]. This questions the validity of the sandwich contact geometry as the surface area available for the water absorption is largely reduced so only short timescales are allowed. In order to increase the surface area for water absorption, the authors used the van der Pauw contact geometry. The results for the sandwich geometry are in agreement with the MDAS model (Figure 1.6(a)) while the van der Pauw conductivity shows a substantial difference from the MDAS model prediction(Figure 1.6(b)).

For the amorphous semiconductor model, several authors tried to predict the energy gap value. Osak et al. measured the current-voltage characteristics of synthetic eumelanin between 283 and 318K [130]. From that, they found two band gap values: $1.58 \mathrm{eV}$ for temperatures above $296 \mathrm{~K}$ and $0.76 \mathrm{eV}$ for temperatures below 296K. They explained this through a connection between the charge conducting and the polarization processes. In later work by Jastrzebska [82] the authors performed a small-range temperature study $(293-343 \mathrm{~K})$. The calculated value for the band gap was found to be between 0.49 and $0.76 \mathrm{eV}$ depending upon the residual water in the sample. The attempt to predict the band gap value was also made by using the Tauc approach (the energy gap is the slope of the plot $(\alpha E)^{1 / 2}$ where $\alpha$ is the measured absorption coefficient and $E$ is the excitation energy [169]). This approach has also resulted in various estimated band gap values, from $3.4 \mathrm{eV}$ [40] to $1.2 \mathrm{eV}$ [65]. The complete uncertainty of the definite value of eumelanin's band gap makes the semiconductor model questionable. 


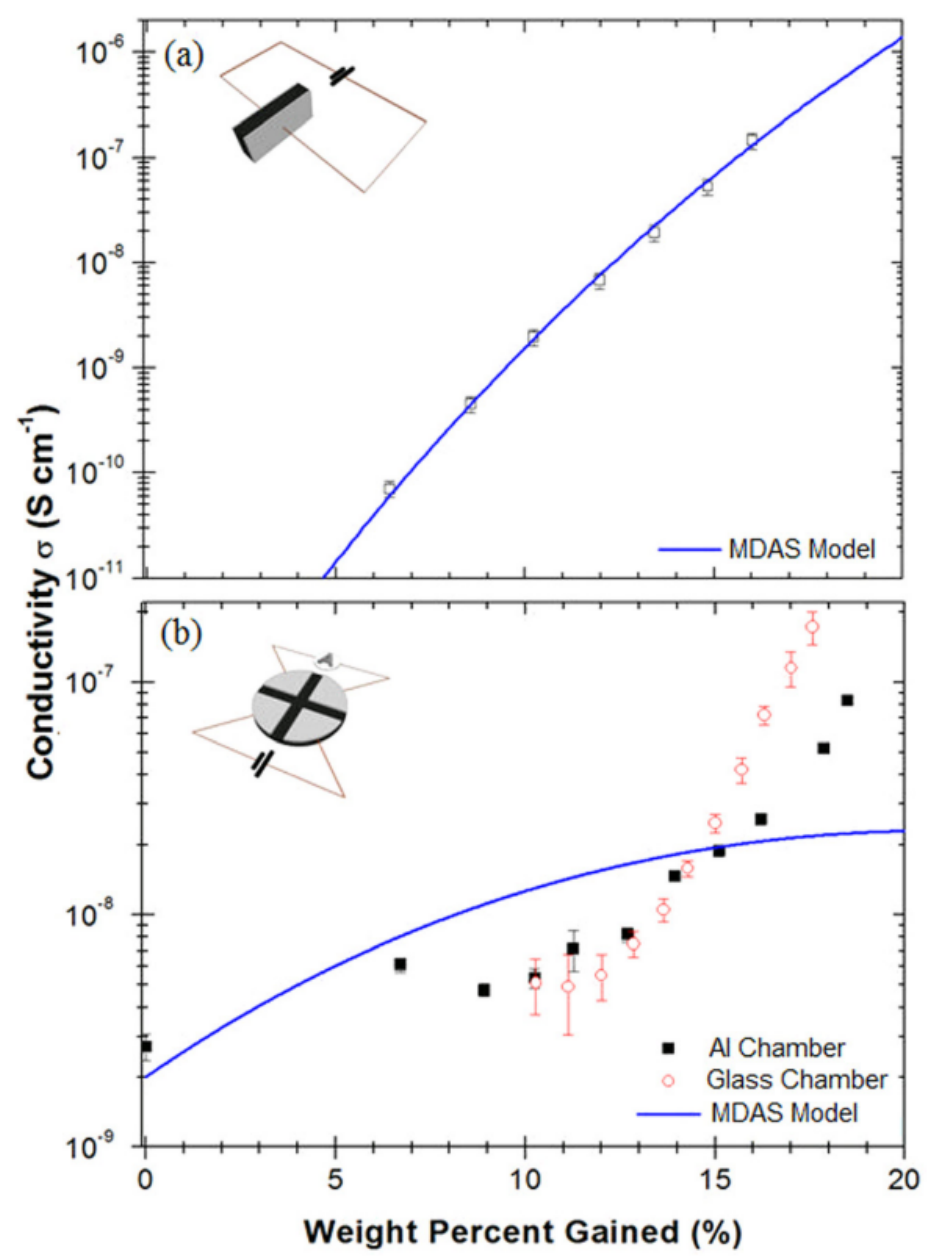

Figure 1.6: Eumelanin conductivity as a function of water content for (a) sandwich contact geometry and (b) van der Pauw contact geometry. Reprinted from [14], with the permission of AIP Publishing.

Photoconductivity properties were first studied by Mostert et al. [14]. He showed that illumination would affect the conductivity of melanin pellets, and the magnitude of this change would be affected by the state of hydration. Figure 1.7 represents two photoconductivity traces at two different hydrations of eumelanin pellets.

The obtained results were qualitatively the same for two different contact geometries. For both sandwich and van der Pauw geometries illumination initiates a photocurrent response generation, which reaches its maximum value within a few 


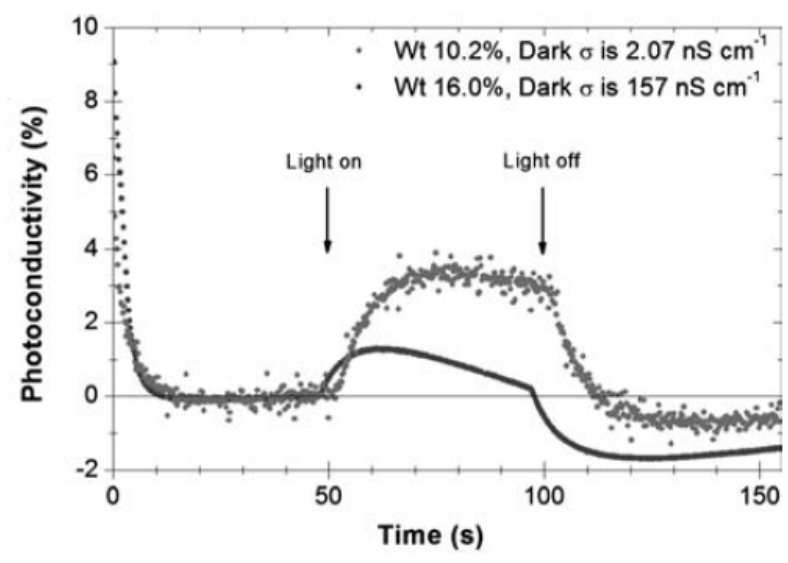

Figure 1.7: Melanin photoconductivity for two different hydration states. Reprinted from [14], with the permission of AIP Publishing.

seconds. The heating of the melanin pellets causes a reduction of water content and, therefore, the authors observed an increase in resistance. Hence, this effect was more pronounced when the samples had more water content in them. After removing the illumination source, photocurrent dissipated in order of a few seconds and then reached a new dark equilibrium [14].

Negative conductivity, produced after removing the illumination, was previously used [40] to support the amorphous semiconductor model, explaining that this phenomenon is due to the trap states in a photo-bandgap of eumelanin which was previously observed in amorphous semiconductors. However, negative photoconductivity has a simpler explanation - the heating and further reducing of water content in the sample was leading to reducing of dark photoconductivity below the initial equilibrium level.

Another inconsistency with the semiconductor model was the observed nonohmic behaviour [130]. It has been shown that synthetic eumelanin satisfies the Ohm law only at low voltages; at high voltages it satisfies the Child law. Additionally, for the semiconductor model to be likely, the secondary structure of eumelanin should be organized as a large highly cross-linked conjugated heteropolymer. On the other hand, as was discussed earlier, there is a wealth of evidence that eumelanin is oligomeric in nature $[59,174]$. Taken together those observations show the 
amorphous semiconductor model fails to explain the properties of eumelanin yet it cannot be ruled out completely without knowing the secondary structure.

\subsubsection{Charge transport in eumelanin}

After proving that MDAS theory is inconsistent the other possible models of charge transport in melanin were considered by Mostert et al. [117]. The authors suggest that an increase of conductivity would arise from either an increase of carrier density or an increase of their mobility. Two different types of carriers were considered: electrons and ions, specifically protons.

To probe the proton mobility the authors used muon spin relaxation technique [15]. Muons are fundamental spin-half particles that are considered as "light protons" (their mass is $1 / 9$ of proton mass) [154]. The experiment results showed that muon hopping rate was constant throughout the hydration range. Therefore, it meant that proton mobility is unaffected by hydration and that hydration adds free radicals to the system. To support these findings, the authors conducted electronic paramagnetic resonance experiments to measure melanin's intrinsic spin population. It was shown that intrinsic spin population decreased with water content, meaning that intrinsic spin population is not linked to the electrical conductivity. It was concluded that no significant carrier density exists in dry melanin pellets.

Upon water absorption, free carriers were produced which drove to the production of free radicals (electrons) and ions (protons). This led to hybrid ionic-electronic behaviour explaining electrical properties of eumelanin observed earlier. However, these studies demonstrated that the proton transfer reactions are important in eumelanin. Hence it raised the question about the kinetics of the proton transfer reactions in eumelanin and whether it can be one of the possible excitation deactivation pathways. 


\subsection{Optical properties}

\subsubsection{Broadband absorption spectrum}

Most organic molecules show distinct peaks in their absorption spectra. Eumelanin, however, has an unusual broadband, monotonic, and featureless spectrum. The broadband nature of the absorption spectrum is most likely related to its efficient photoprotective mechanism - rising from NIR to UV region the spectrum has no gaps allowing the harmful irradiation to penetrate through the skin layers and interact with the cells while the peaked spectrum would transmit photons at certain wavelengths.

To this day, the broadband nature of eumelanin's absorption spectrum remains the subject of scientific debates [30]. The origin can be explained with several alternative models which will be discussed below.

\section{Scattering effect on the broadband shape of absorption}

Wolbarsht et al. [179] first suggested that the true absorption spectrum of eumelanin might be obtained by subtracting the effects of Rayleigh scattering (by molecules themselves) and Mie scattering (by melanosomes). However, Riesz et al. [148] measured the scattering coefficient of eumelanin solutions as a function of wavelength over the UV range. In this part of the spectrum the scattering effect is more significant because the Rayleigh scattering, for small particles (with the radii less than $50 \mathrm{~nm}$ ), has a $\lambda^{-4}$ dependence and Mie scattering (for larger particles) is wavelength independent. The results (Figure 1.8) showed that scattering contributes less than $6 \%$ of the optical density within the measured range. This means that the absorption spectrum of eumelanin is likely due to its electronic properties.

\section{Chemical disorder model}

This alternative model, proposed by Paul Meredith group [111], suggests that the chemical disorder of eumelanin pigment is responsible for the broadband shape of the absorption spectrum. Numerous experimental evidence [30,111, 113] of the 


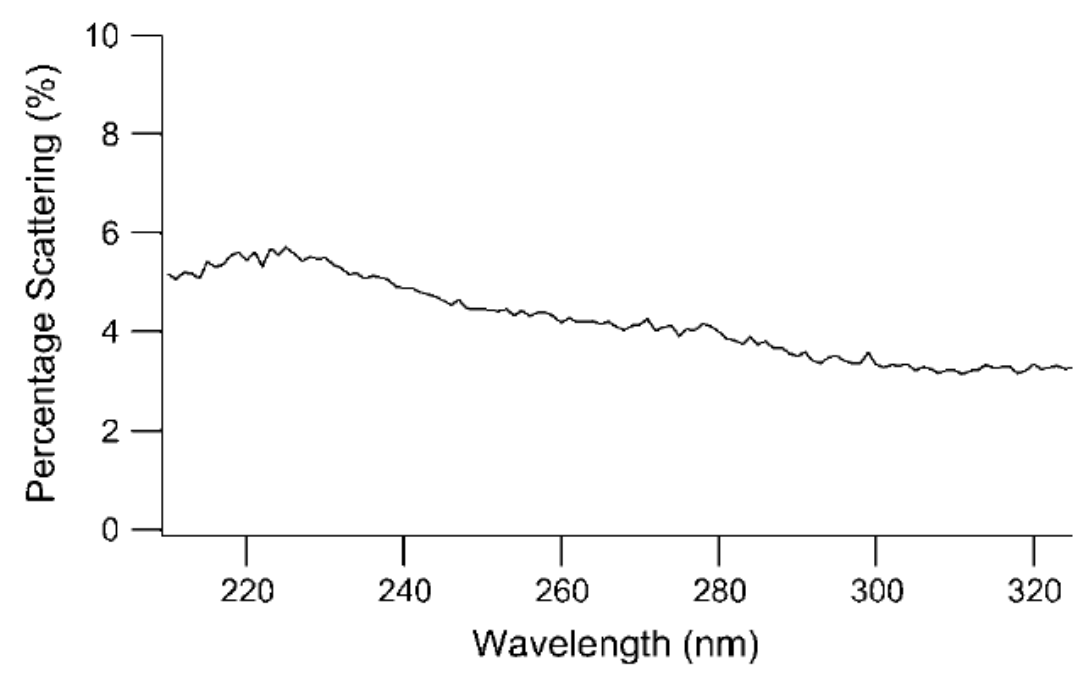

Figure 1.8: The scattering coefficient as a percentage of the total optical density. Reprinted from [148] with permission from The Biophysical Society. Published by Elsevier.

predominance of the disorder in the system makes this model the most popular and well accepted to this day.

The idea of the chemical disorder model lies in the assumption that the broadband absorption spectrum of eumelanin is in fact the superposition of the absorption from consistent oligomers ( $<10$ monomers) or so-called chromophores. In this model the chromophores are randomly distributed in the system, each having a distinct peak in different regions of the spectra, with their absorption peaks overlapped. This results in the observed broadband shape of the pigment's absorption spectrum.

Eumelanin is known to consist of DHI and DHICA building blocks and their different redox forms. The density functional theory (DFT) calculations on both DHI and DHICA performed by Tran et al. [172] suggest that different redox states of those building blocks have different HOMO-LUMO gaps and, therefore, different absorption spectra. Namely, the calculations showed that DHICA oligomers' HOMO-LUMO gaps are red shifted from that of the monomer. In addition, the oxidized hydroquinone, indolequinone and semiquinone states are predicted to be present in solutions in quantities high enough to contribute to the eumelanin's ab- 
sorption spectrum. Those states are shown in Figures 1.9 and 1.10. The predicted HOMO-LUMO gap for indolequinone was significantly redshifted to $615 \mathrm{~nm}$ from DHI hydroquinone state's energy gap at $343 \mathrm{~nm}$. The DHI semiquinone shows even larger shift to $1110 \mathrm{~nm}$ [138]. For the DHICA building block, the later study of the equivalent redox states show the predicted HOMO-LUMO values at $408 \mathrm{~nm}$ for hydroquinone, $634 \mathrm{~nm}$ for indolequinone and $1127 \mathrm{~nm}$ for semiquinone [137]. From those values, it can be concluded that the addition of the carboxylic acid group in DHICA has a significant effect on the physical properties of those molecules, in particular, the HOMO-LUMO gap. This difference for the various monomers can lead to a large range of HOMO-LUMO gaps in the eumelanin oligomers which can result in the broad shape of the resulting eumelanin absorption spectrum.<smiles>Oc1cc2cc[nH]c2cc1O</smiles>

Hydroquinone<smiles>O=C1C=c2cc[nH]c2=CC1=O</smiles>

Indolequinone<smiles>O=C1C=C2C=CN=C2C=C1O</smiles>

Semiquinone

Figure 1.9: DHI redox forms (adapted from [138]).

In their later study, Meredith et al. [111] noted that the ground to excited states transitions in organic chromophores in solutions are characterised by inhomogeneously broadened Gaussian peaks. The linear superposition of those peaks may result in the broad absorption shape of the spectrum. In fact, the broadband shape can be reproduced by a superposition of a relatively small number of Gaussian peaks (Figure 1.11). As was described in Meredith paper, the peak spacing is $50 \mathrm{~nm}$ and full width at half maxima is $90 \mathrm{~nm}$ (typical value for organic chromophore in a polar solvent). The intensity of the Gaussians was given by $I=\exp (-\lambda / C)$ where 
<smiles>O=C(O)c1cc2cc(O)c(O)cc2[nH]1</smiles>

Hydroquinone<smiles>O=C1C=c2cc(C(=O)O)[nH]c2=CC1=O</smiles>

Indolequinone<smiles>O=C(O)C1=CC2=CC(=O)C(O)=CC2=N1</smiles>

Semiquinone

Figure 1.10: Equivalent DHICA redox forms (adapted from [137]).

$\lambda$ is a peak wavelength and $C$ is a free constant which was taken to be $200 \mathrm{~nm}$. To reproduce eumelanin's spectrum, the exponential envelope has been applied to the Gaussian peaks. This shows that the broadband spectrum can be reproduced as a summation of narrow absorption bands of many different oligomers. As was shown earlier in this section, different redox forms of the main building blocks DHI and DHICA are producing a different range of HOMO-LUMO gaps in UV, visible, and IR spectral range. Thus, it shows that the group of the chemically distinct species can recreate the broadband and monotonic behaviour of the absorption spectrum.

Kaxiras et al. [87] performed time-dependent DFT calculations of the absorption spectrum resulting from the superposition of the spectrum of dominant tetramers (Figure 1.12). It is clearly observed that the superposition of distinct peaks creates a broad and featureless spectrum. The sharp features of the tetramers' absorption peaks are eliminated when the average is taken, which serves as another indicator of the chemical disorder model. The peak around $350 \mathrm{~nm}$ was also observed in previous studies [172] for eumelanin structures with the incomplete oxidative polymerization of DHICA. The range below $250 \mathrm{~nm}$ has not been experimentally observed due to the high water absorption at the higher energies, possibly explaining why the peak at $200 \mathrm{~nm}$ has not been detected before.

Additionally, the violation of Kasha's rule (the photon emission will only occur 


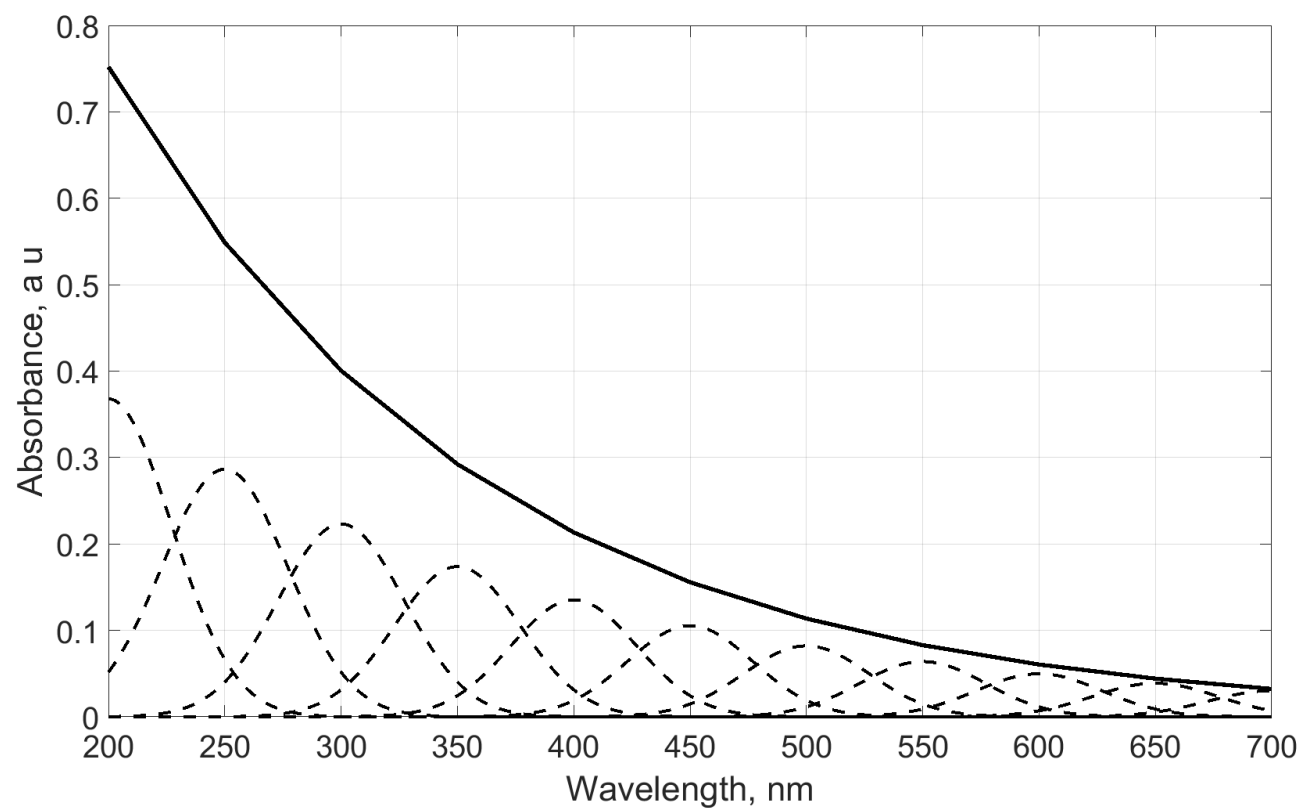

Figure 1.11: Simulation of eumelanin broadband absorption spectrum using a linear combination of 11 Gaussians. Reproduced using the approach from [111]. The exponential envelope is shifted for clarity.

from the lowest excited state; so that the excitation will only change in intensity as the excitation wavelength changes [86, 97]), observed by Meredith and Riesz [112], also supports the chemical disorder model. They demonstrated that the shape and the peak position of the photo-emission spectrum are changing with changing the excitation wavelength. One can suggest that this is due to the extreme disorder which may produce discrete states across the energy range.

Together with the ability of this model to reproduce the broadband featureless spectrum, this makes the chemical disorder model more trustworthy than others considered before. Although this theory best explains eumelanin's behaviour, it does provide a physical explanation why the absorption spectrum increases monotonically towards UV [30]. Therefore a better understanding of the secondary structure of eumelanin is required to learn about eumelanin's ingenious properties. 


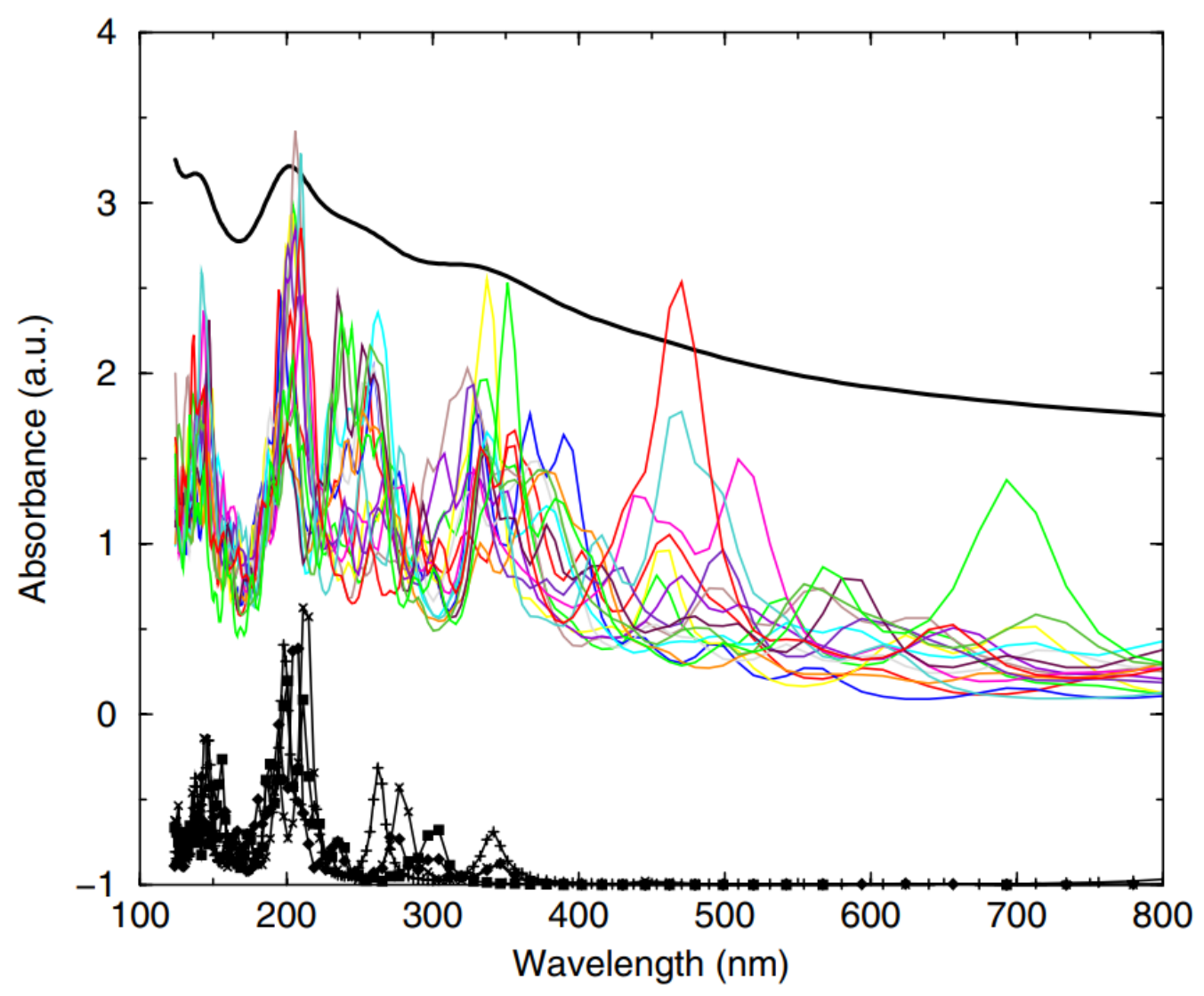

Figure 1.12: Absorption spectrum of eumelanin as a result of the superposition of the dominant tetramers spectrum (thick black line, shifted up by 1.5 units for clarity). Tetramers' spectra are shown by the thin coloured lines, monomers' spectra are shown by the black lines (shifted down by 1 unit for clarity). Reprinted with permission from [87]. Copyright (2006) by the American Physical Society.

\section{Excitonic model}

The chemical disorder model, albeit very convincing, is still insufficient to fully explain eumelanin's optical properties. The stacked oligomer model, confirmed with several experiments, claims that eumelanin is made of oligomeric scaffolds with an interlayer distance of 3-4 $\AA$. It was proved that the interaction between eumelanin protomolecules are strong enough to significantly affect the spectrum [163] which is not consistent with the superposition principle. Furthermore, the chemical dis- 
order model is unable to explain the monotonic increase of eumelanin absorption spectrum [30].

In 2014 Chen et al. [30] conducted a series of theoretical studies on a variety of eumelanin molecular structures [32, 33, 46, 87]. Their results showed that the spectra of eumelanin protomolecules are very dependent on the aggregate size. This motivated the authors not to use a simple superposition principle, that ignores excitonic couplings among eumelanin chromophores.

Additionally, the authors showed that the excitonic coupling between eumelanin protomolecules results in an increase of oscillator strength toward UV spectral range in the eumelanin absorption spectrum. For large-scale systems, when the absorption spectra of the aggregate structures were simulated with the proper account of the excitonic coupling in agreement with Frenkel exciton model, the resulting spectra were demonstrating characteristics, similar to ones observed in eumelanin, namely, monotonically increased broadband spectrum, rising towards UV. It was observed that the gas-phase absorption peaks of eumelanin protomolecules are redistributed when they stack together and allowed to interact via excitons. This leads to a featureless spectrum that is resembled closely the one observed experimentally [30].

By modelling the distribution of the interlayer distance of adjacent molecules, as well as dihedral angles between them supported the importance of excitonic effects on the broadband absorption of eumelanin. It was shown that eumelanin is a rotationally disordered structure, i.e. the stacked molecules have no favourite rotational configuration. From these calculations it was concluded that in the large scale system both geometric order and geometric disorder are observed coincidentally. The geometric order refers to stacking on planar eumelanin protomolecules, while geometric disorder is indicating the lack of preference of the rotational degrees of freedom between adjacent molecules. Both those properties lead to the characteristics of broadband absorption of eumelanin - geometric order induces excitonic couplings among eumelanin protomolecules and geometric disorder leads to scattering the oscillator strength (or absorption intensity) across the full range of the band [30].

The inclusion of excitonic interactions was crucial in accounting for eumelanin broadband absorption spectrum shape. Excitonic couplings are responsible for spec- 
tral broadening as well as the rise of the absorption spectra towards high energy spectral range. This was an important addition to the chemical disorder model, showing that excitonic effects play a crucial role in explaining the broadband nature of eumelanin aggregates' absorption spectra.

\subsubsection{Photoluminescence emission}

As was briefly mentioned before, eumelanin seemingly demonstrates a violation of Kasha's rule - the emitted energies show a dependence on the material properties rather than on the excitation energy. It was shown that eumelanin possesses a wavelength-dependent steady-state emission (Figure 1.13). This served as evidence for the chemical disorder model - by tuning the excitation wavelength we selectively excite distinct chromophores of eumelanin, that are overlapping and producing the broadband absorption spectrum. Nighswander-Rempel et al. also noticed a slight peak at $380 \mathrm{~nm}$, appearing in uncorrected emission spectra [122]. This was attributed to electronically isolated DHICA units on the periphery of the molecule.

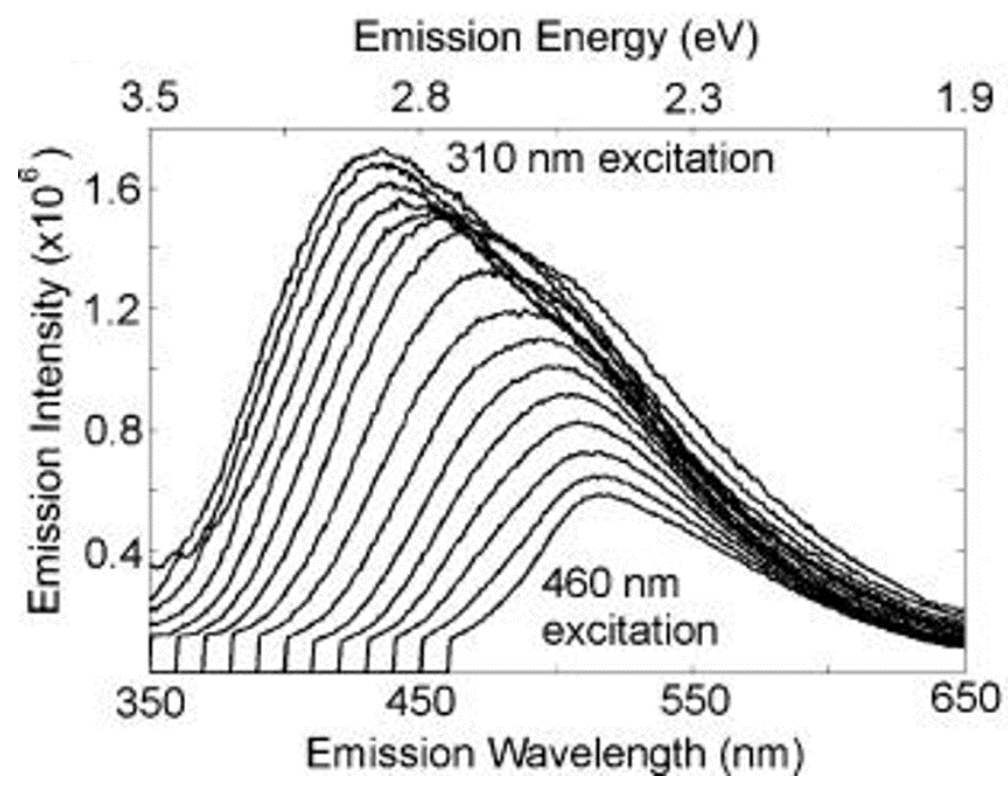

Figure 1.13: Excitation spectra showing wavelength-dependent emission. Reprinted from [123], with the permission of AIP Publishing. 
Eumelanin possesses a very low radiative quantum yield (ratio of emitted photons to excited photons). Meredith and Riesz [112] have reported radiative relaxation quantum yield value to be between $5 * 10^{-4}$ and $7 * 10^{-4}$ which is $<0.1 \%$ of the excitation. Additionally, it was demonstrated, that unlike for many other substances, for which quantum yield is constant with excitation wavelength, for eumelanin this value is highly excitation-dependent and varies over the range $250-500 \mathrm{~nm}$ [123].

Since eumelanin's radiative quantum yield is so low, it was important to understand where does the excess of the energy go. A wavelength-dependent photoacoustic study by Forest and Simon [55] for eumelanin in physiological conditions ( $\mathrm{pH}$ 7.2) showed that the majority of the absorbed energy is dissipated as heat through nonradiative ultrafast channels.

\subsection{Photoprotective properties}

UV irradiation from the Sun consists mainly of 95\% of UV-A (400-315 nm) and 5\% of UV-B (315-280 nm) light. UV-C radiation (280-100nm) is absorbed by Earth's atmosphere [20]. Exposure to the sun can cause photo-aging (e.g. wrinkles), erythema (or sunburn) photo-damage of DNA, several skin tumours such as basal cell carcinoma, squamous cell carcinoma and malignant melanoma, the most and aggressive type of skin cancer [88]. It is believed that UV-B radiation, when absorbed directly by DNA, is responsible for DNA breakage and tumour formation. However, UV-A photons which can penetrate deeper into the skin, are involved in the generation of reactive oxygen species such as singlet oxygen, superoxide, hydrogen peroxide and hydroxyl free radicals [34] which can be a cause of malignant melanoma and other types of skin cancer.

It is assumed that skin pigmentation is the skin's primary photoprotective mechanism. In fact, epidemiological research confirmed that people with darker skin are less susceptible to the risk of skin cancer than lighter-skinned individuals, which correlates with a higher concentration of eumelanin and lower skin cancer rates $[21,62]$. The melanosomes in dark skin are resistant to degradation after UV expo- 
sure while the fair skin melanosomes degrade fast and therefore produce substantially less photoprotection against the UV-induced photodamage [91]. That means that the individuals with higher eumelanin concentrations have better photoprotection against the high energy UV photons.

Skin pigmentation is widely believed to be an adaptation to protect folic acid from UV radiation. It was hypothesised that the melanin pigmentation balances folate preservation. Folate is proposed as cancer prevention target for its role to provide presursors for DNA repair [177].

Albeit the fact that eumelanin's photoprotective properties are well-discovered and undeniable, the pigment can also have toxic properties after the UV exposure and act as a photosentisizer after the exposure to UV light [20, 34, 69, 129]. As a photosensitizer, melanin can participate in the singlet oxygen generation which is highly toxic and leads to the cells apoptosis. However, it has been shown that among the melanins, it is pheomelanin, and not eumelanin that will more likely act as a photosensitizer following the UV exposure. Pheomelanin is demonstrated to be able to generate more singlet oxygen for longer periods than eumelanin $[68,167]$.

The photoprotection properties of eumelanin originate in two intrinsic pigment features - the broadband absorption spectrum, monotonically rising towards UV region of the spectra where the photodamage is the most severe; and the ultrafast character of the energy dissipation allowing to transfer most of the harmful UV energy to the heat before the damage can occur. Although eumelanin's role as an efficient natural sunscreen is well accepted, there is no clear understanding of how UV radiation is dissipated to heat after photo-excitation.

A low radiative quantum yield that was discussed in section 1.3.2 indicates that most of the absorbed energy is released nonradiatively. Radiationless transitions are ultrafast and allow to dissipate $99.9 \%$ of the harmful UV radiation before the molecules in the body can be damaged. Therefore, the study of nonradiative mechanisms is crucial for understanding the origin of eumelanin's unique photoprotection properties. 


\subsection{Ultrafast nonradiative relaxation dynamics}

As was shown before, the radiative relaxation pathways are playing a minor role in the photo-dynamics of eumelanin which means that nonradiative, rapid relaxation pathways are prevalent. The nature of those pathways as well as the minimal functional unit responsible for ultrafast UV dissipation remains obscure. In order to investigate those pathways, photoacoustic studies have been conducted [124]. These studies revealed rapid nonradiative dissipation of the absorbed energy into heat in sub-ns time scale [124].

Previous transient absorption (TA) study of nonradiative relaxation dynamics revealed that the rapid ground state repopulation follows excitation [126]. Comparison with previous studies showed that the ground state repopulation is the dominant mechanism of energy dissipation. The ground state repopulation dynamics revealed the non-exponential behaviour of the excited-state decay (Figure 1.14). In fact, four exponential time constants were needed to fit the decay dynamics. This indicates that several competitive dissipation pathways exist. However, the observed emission data did not provide information about the nature of those dissipation pathways. Nonexponentiality of the decays may also serve as a support for the chemical disorder model as those time constants could be related to discrete heterogeneously distributed chromophores. The observed dynamics also had a multiexponential character.

For better understanding the energy dissipation in eumelanin pigment it is important to identify the minimal functional unit of UV dissipation and define the main process responsible for the excited state dynamics. For elucidating the excited state properties of each of the main eumelanin blocks, DHI and DHICA in buffer solutions at different $\mathrm{pH}$ were investigated [37, 38, 60, 61, 73, 110].

In the later work of Corani et al. [37], the ultrafast sub-picosecond fluorescence decay was observed in DHICA dimers and showed similar lifetime values to that of DHICA polymers (Figure 1.15). Dimers showed more than 1000 times more efficient excited-state decay than monomers. These observations imply that the ultrafast excited-state decay process that takes place in dimers does not evolve further 


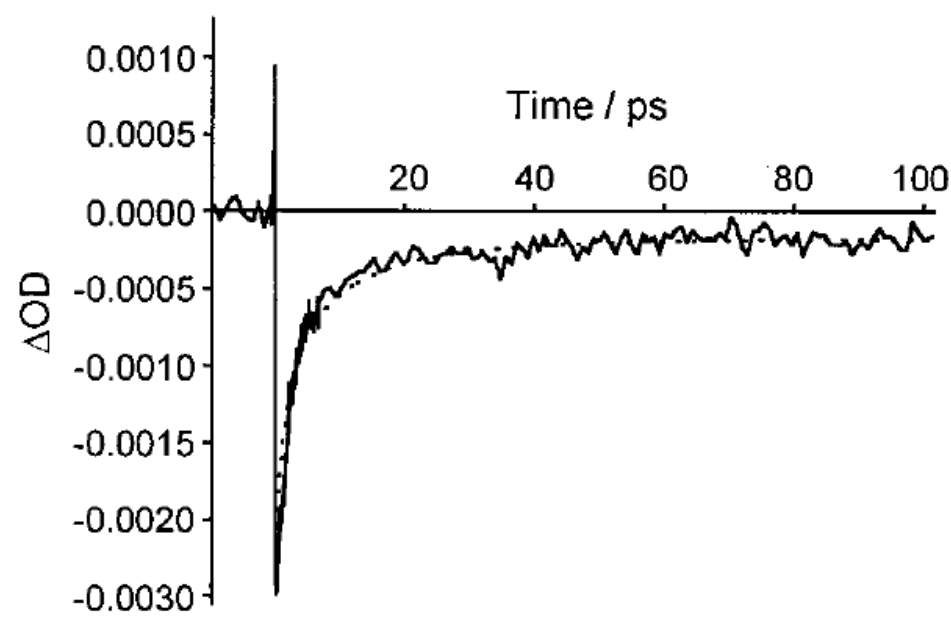

Figure 1.14: Degenerate $350 \mathrm{~nm}$ pump-probe transient absorption data. Reprinted with permission from [126]. Copyright (C) 2001, American Chemical Society.

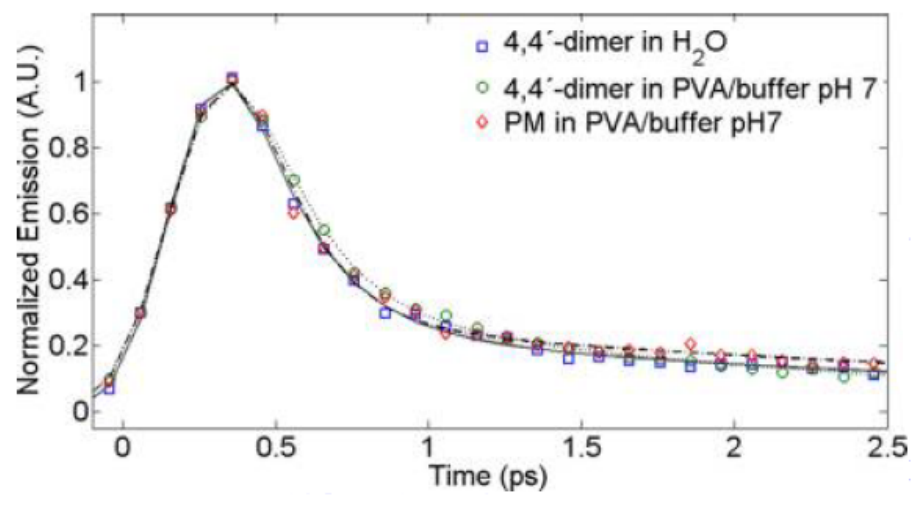

Figure 1.15: Fluorescence up-conversion decays of DHICA dimer in water and polyvinyl alcohol buffer and DHICA polymer (PM) in polyvinyl alcohol buffer. Reprinted with permission from [37]. Copyright (C) 2014, American Chemical Society. 
through polymerization. From that, it can be concluded that DHICA dimers provide the minimal functional unit for an ultrafast energy dissipation which leads to a conclusion that the photoprotective function is a property of DHICA. However, it has been shown [127] that DHI can also exhibit short-lived (about 103 ps) excited states. Therefore, the role of the main eumelanin building blocks in the photoprotection mechanism requires further investigation.

In the following sections we will go into more details about some possible excitedstate decay mechanisms.

\subsubsection{Excitation energy transfer between the chromophores}

Electromagnetic coupling between neighbouring chromophores creates delocalised excitations - electronic excited states whose size and energy depend on the geometrical configuration of the constituent chromophores [156]. Excitation energy transfer (EET) has been previously established as a decay mechanism in several aggregates of organic molecules [51, 105], so one speculates that the delocalized excited state might give rise to an ultrafast energy relaxation due to exciton interactions in eumelanin.

EET in aggregates represents a two-step process (Figure 1.16). First, two molecules are initially at their first excited states $S_{1}$. To create an excitation they have to move closer to each other so that they can create a higher excited state $S_{n}$ at one molecule (exciton fusion). The energy transfer at the higher excited state occurs by using the energy of the other molecule which becomes de-excited. Then, an ultrafast internal conversion process brings back the first molecule to the $S_{1}$ state.

To better investigate the nature of the ultrafast relaxation in the key building block, DHICA and DHICA-based model compounds (dimers, trimers, oligomers) were investigated by Corani et al. [37]. Absorption spectra of the samples measured in $\mathrm{pH} 2.5$ and $\mathrm{pH} 7$ show similarity to that of the DHICA-containing natural eumelanin. Upon deprotonation of the carboxylic group at $\mathrm{pH} 7$ the blue shift is observed in monomer spectra. This shift becomes smaller for oligomeric structures. For the dimers, the red shift was observed relative to the absorption maximum of the DHICA monomer at both $\mathrm{pH}$ values and trimers show the red shift at $\mathrm{pH}$ 7. Those 


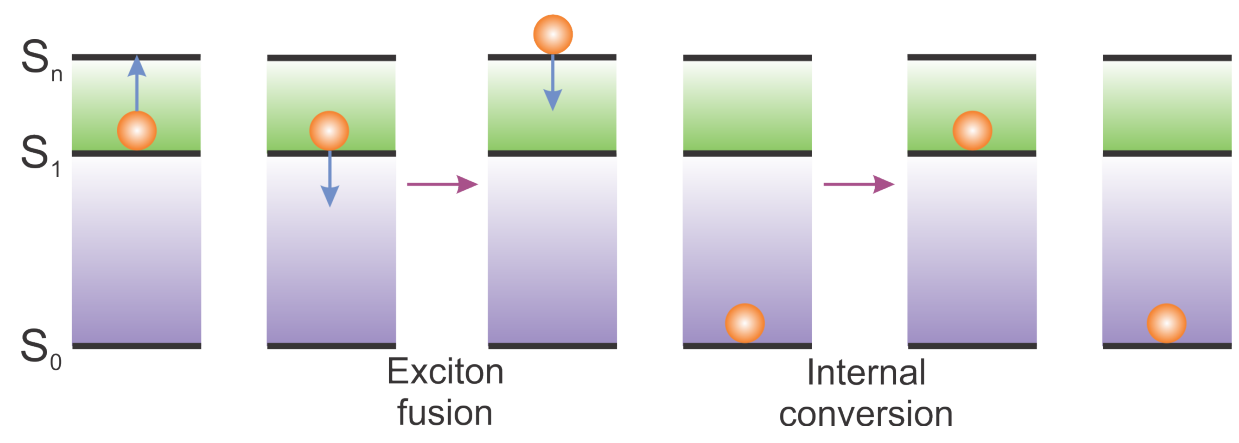

Figure 1.16: EET process between two chromophores. Adapted from [105].

shifts can indicate a certain degree of electron delocalization of excitonic interactions between DHICA monomers.

When individual units of DHICA create oligomeric aggregates they come close to each other so that they can create excitons interaction and further exciton-exciton annihilation. To investigate whether the observed red shift in the oligomer absorption spectra is due to excitonic nature, the absorption of model oligomers was measured and compared with the spectra of monomers [37]. It was expected to observe a broadening of the oligomeric spectra compared to monomeric. Instead, the spectra with more or less the same spectral shape and a small red shift were detected. From that, it was concluded that the exciton relaxation involving delocalized states is not a superior mechanism for ultrafast energy dissipation.

Excitation energy transfer was also studied in eumelanin aggregates. The spectral hole burning spectroscopy measurements [41, 80] are very useful for studying the multichromophore system when the absorption of the subset of the chromophores differs from the absorption of the full system [81], which is the case for eumelanin, assuming that the chemical disorder model is correct. The behaviour of the ground state bleach can indicate different possibilities for the energy dissipation in eumelanin. Kohl et al. [92] performed the spectral hole burning spectroscopy on the synthetic eumelanin and revealed that the ground state bleach (GSB) did not experience red shift with time. That indicated that the excitation energy transfer (EET) between chromophores was highly unlikely to happen because one would observe GSB to redshift as the energy funnels from excited chromophore to lower energy 


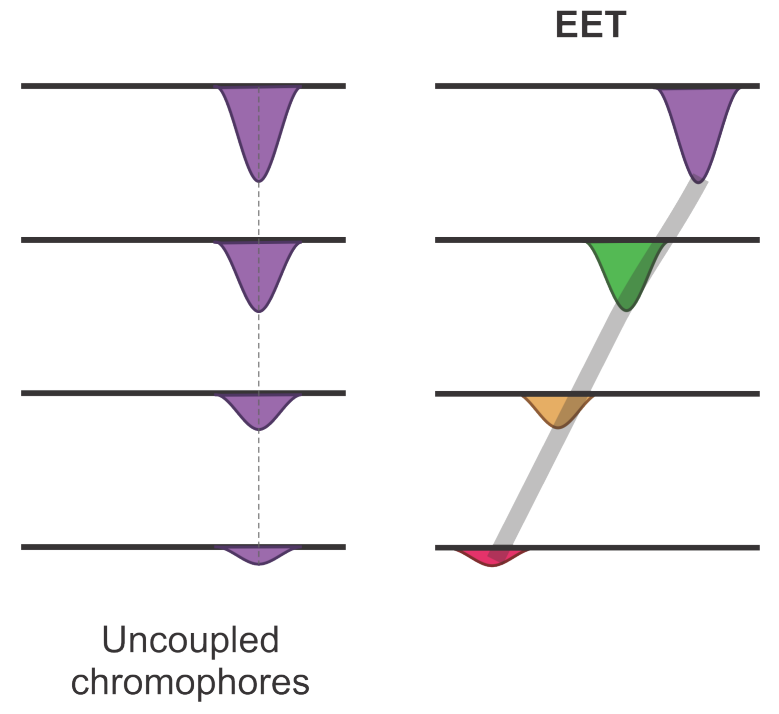

Figure 1.17: Energy migration by excited energy transfer. Adapted from [92].

excited chromophore (Figure 1.17). However, GSB peaks from the lower energy excited chromophores can appear as a result of an energy transfer from a higher energy excited chromophore and get summed up with a photoinduced absorption (PIA) peak from another chromophore. Therefore, another proof is needed to rule out the energy transfer hypothesis.

\subsubsection{Excited state proton transfer}

The proton transfer (or the $\mathrm{H}^{+}$ion transfer) has been assigned as one of the most important chemical reactions [25]. In agreement with thermodynamics, the protoncontaining molecules in their excited states are stronger acids than in the ground state [157] if the absorption or emission spectrum of the conjugated base is redshifted relative to that of the photoacid (photoexcited molecule) [37, 171]. If those conditions are met the excited-state proton transfer (ESPT) to the solvent can occur. Figure 1.18 shows the proton transfer process in the excited state.

An intramolecular excited-state proton transfer (ESIPT) occurs when the molecule has proton donor and proton acceptor atoms linked with an intramolecular hydrogen bond that is formed in the ground state [45]. This process has also been proposed as a possible mechanism of the ultrafast energy dissipation in building 


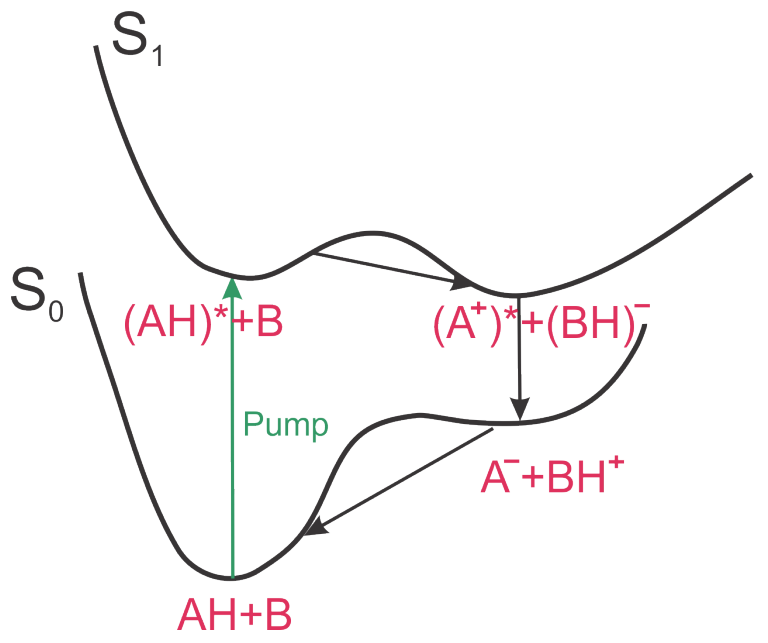

Figure 1.18: Excited state proton transfer. The pump induces the $A H$ molecule to its excited state $(A H)^{*}$ leading the an electronic redistribution which results in a proton transfer from $(A H)^{*}$ to B. Adapted from [1].

blocks of organic molecules [73]. The time-resolved emission studies [158] on QCy9 in water showed that the ESIPT process happens on a very fast time scale of $100 \mathrm{fs}$ range. That was also shown before for the eumelanin model [110]. From the charge transport studies [117], it was discovered that the protons are one of the main carriers in eumelanin. However, the study of the pigment as a conjugated acid and base is complicated by its high insolubility in the most known solvents. Therefore, it was important to apply the bottom-up approach by studying the photochemistry of eumelanin building blocks DHICA and DHI.

It has been observed that in the case of electronically isolated DHICA the emission spectrum did not mirror the absorption spectrum (Figure 1.19). This violates the so-called mirror-image rule [90] (symmetry between absorption and emission spectra of organic chromophores). From the Figure 1.19 we observe the doublepeaked absorption spectrum of DHICA - the main peak at $3.8 \mathrm{eV}$ and a shoulder at $4.2 \mathrm{eV}$ [128]. These two peaks are attributed to $S_{0}-S_{1}$ and $S_{0}-S_{2}$ transitions. The resulting species then react through adiabatic (from $S_{2}$ ) and non-adiabatic (from $S_{1}$ ) ESPT to form $S_{1}$ state of the deprotonated DHICA form. This form then decays by photon emission [128]. This results in single-peaked emission and mirror-image 
rule violation.

Later studies [60] revealed that the fluorescence lifetime decreased from organic to acidic aqueous solvents of DHICA monomers and oligomers (Figure 1.20). This observation led to a hypothesis that the proton transfer process is responsible for the ultrafast energy dissipation in DHICA. The proposed processes are illustrated in Figure 1.21.

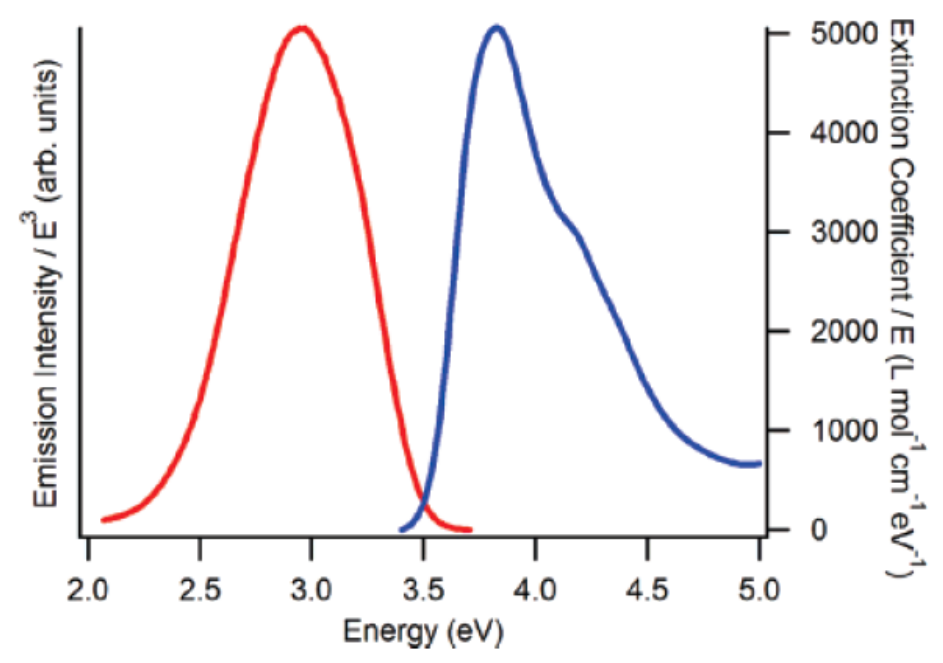

Figure 1.19: Scaled absorption (blue) and emission (red) spectra of DHICA in aqueous solution. Reprinted with permission from [128]. Copyright (C) 2007, American Chemical Society.

From the discussion above it can be concluded that DHICA oligomers might be a main component of eumelanin responsible for ultrafast excited state relaxation via excited state proton transfer mechanism which means that it plays a key role in the photoprotection mechanism. However, the role of DHI was not that clear. It has been noticed that, apart from short-lived excited states in monomeric forms, DHI structures exhibit long-lived excited states [60] which lead to the radical formation following the UV-radiation. Therefore it was investigated whether an efficient excited state deactivation through ESPT can also happen with DHI [127]. It has been shown that similar to DHICA, DHI exhibits $\mathrm{pH}$-dependent ultrafast excited state deactivation lifetime. The process is initiated through proton-coupled electron transfer (PCET) and a formation of solvated electron which recombines with deprotonated 


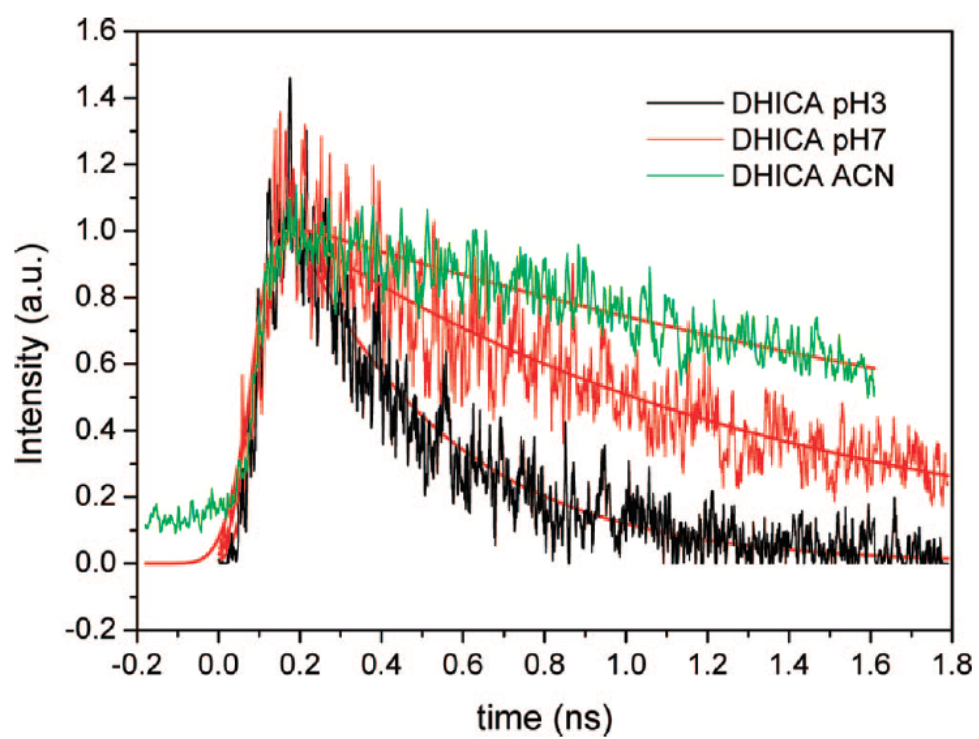

Figure 1.20: Fluorescence decay of DHICA at $\mathrm{pH} 3, \mathrm{pH} 7$ and in acetonitrile. Reprinted with permission from [128]. Copyright (C) 2007, American Chemical Society.

DHI species after the relaxation to the ground state by the internal conversion process.

\subsubsection{Charge transfer states recombination}

Transient hole burning spectroscopy was very helpful in excluding excited energy transfer between the chromophores (yet an additional proof is still required) [92]. The authors suggested that eumelanin is capable of creating the charge transfer (CT) states. These states can form intramolecularly, or, in other words, between the chromophores of eumelanin. The oligomeric model of the pigment structure would support that idea - the protomolecules, stacked via $\pi-\pi$ interactions have the close interlayer separation thus facilitating the formation of the CT excitons. The authors suggested, based on the spectral hole burning spectroscopy measurements, that interchromophore $\mathrm{CT}$ states formation and their further recombination to the ground state takes place in the synthetic eumelanin [92].

In the case of the charge transfer between two chromophores, the bleach from both initially excited and the second chromophores would appear. If the photoex- 


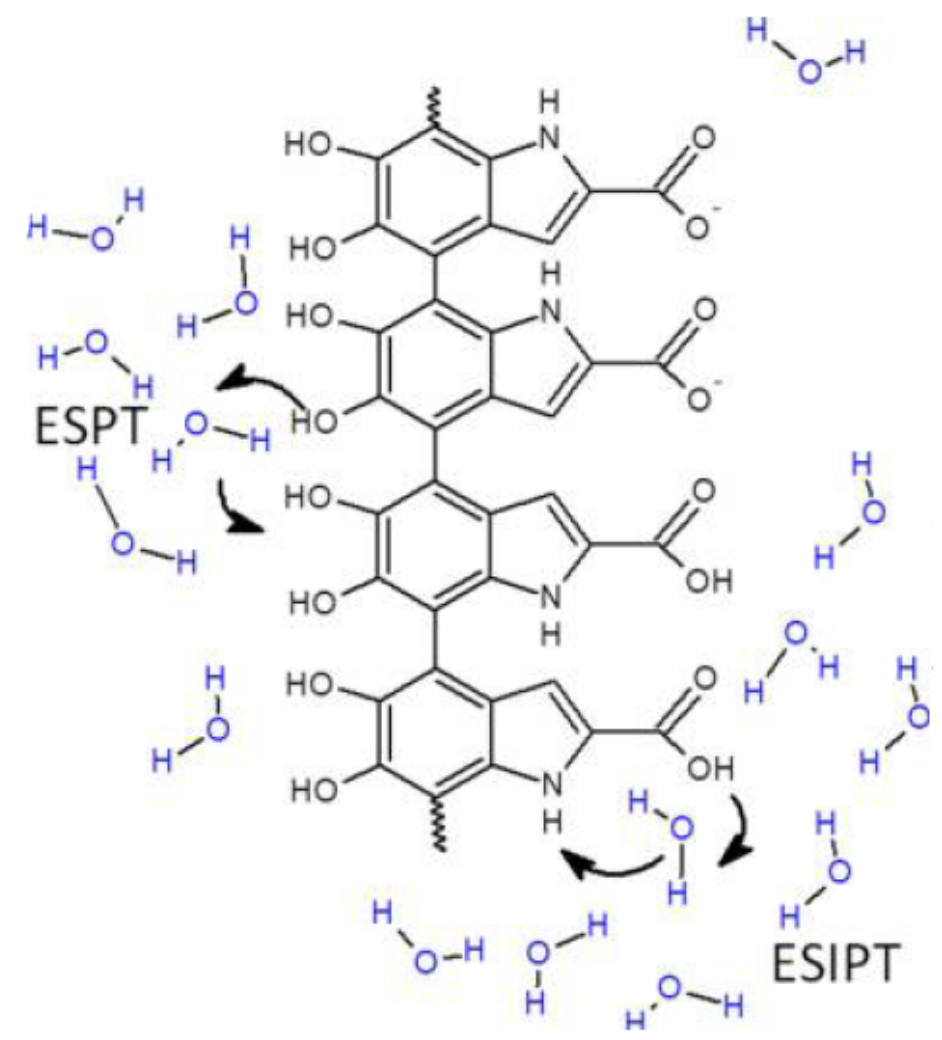

Figure 1.21: Summary of the ultrafast excited state deactivation of the DHICA oligomer via excited-state proton transfer processes. Reprinted with permission from [37]. Copyright (C) 2014, American Chemical Society.

cited molecule underwent charge transfer with many chromophores, the bleaches from all of them will appear next to the initially excited chromophore bleach (Figure 1.22 , on the right). On the scheme it is demonstrated that the initially excited chromophore has the strongest bleach. The charge recombination restores the ground state for all the chromophores so that they decay together.

To rule out the energy transfer between the chromophores with similar transition energies the authors performed the polarization anisotropy experiments. The anisotropy theory predicts that in the case of the perpendicular pump and probe polarizations, the signal will be three times lower than for the case of the parallel polarizations when there is no depolarization in the bleached chromophores [180]. The results showed in Figure 1.23 indicate that the bleach signal did not lose the 


\section{CT excitons}

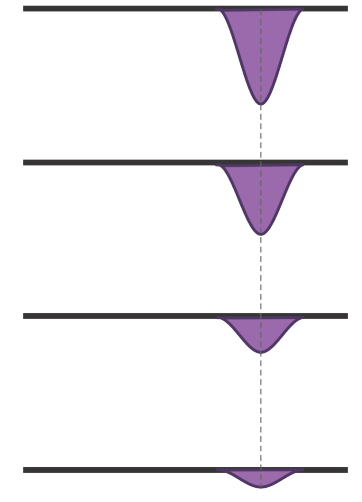

Uncoupled chromophores

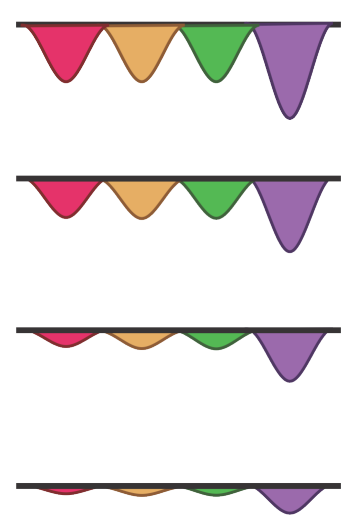

Coupled chromophores

Figure 1.22: Ground state bleach dynamics from possible excited-state decay pathways. The colors of the peaks represent the different absorption energies - from high (violet) to low (red). Adapted from [92].

initial polarization which indicated that the initially excited chromophore's transition dipole moment did not reorient after the excitation [84]. The photoinduced absorption signal, however, quickly lost the memory of the initial excitation polarization. As an explanation for that, the authors suggested the immobile CT states [8] that are formed either intramolecularly or as a result of the charge transfer between chromophores.

As an additional evidence favouring the CT states hypothesis authors stated that the photoinduced absorption bands of the different chromophores showed a relatively similar spectral shape because the non-interacting chromophores would show their own characteristic spectra. The authors believed that the nearly identical spectral response indicated the interaction between the excited states of the chromophores which could be a CT state formation.

We believe, however, that this assumption is incomplete as the authors have not taken the DHICA or DHI building blocks into consideration for having a bottomup approach to study the pigment's complex nature. It was already demonstrated $[37,38]$ that DHICA plays a significant role in eumelanin photophysics and hence it 


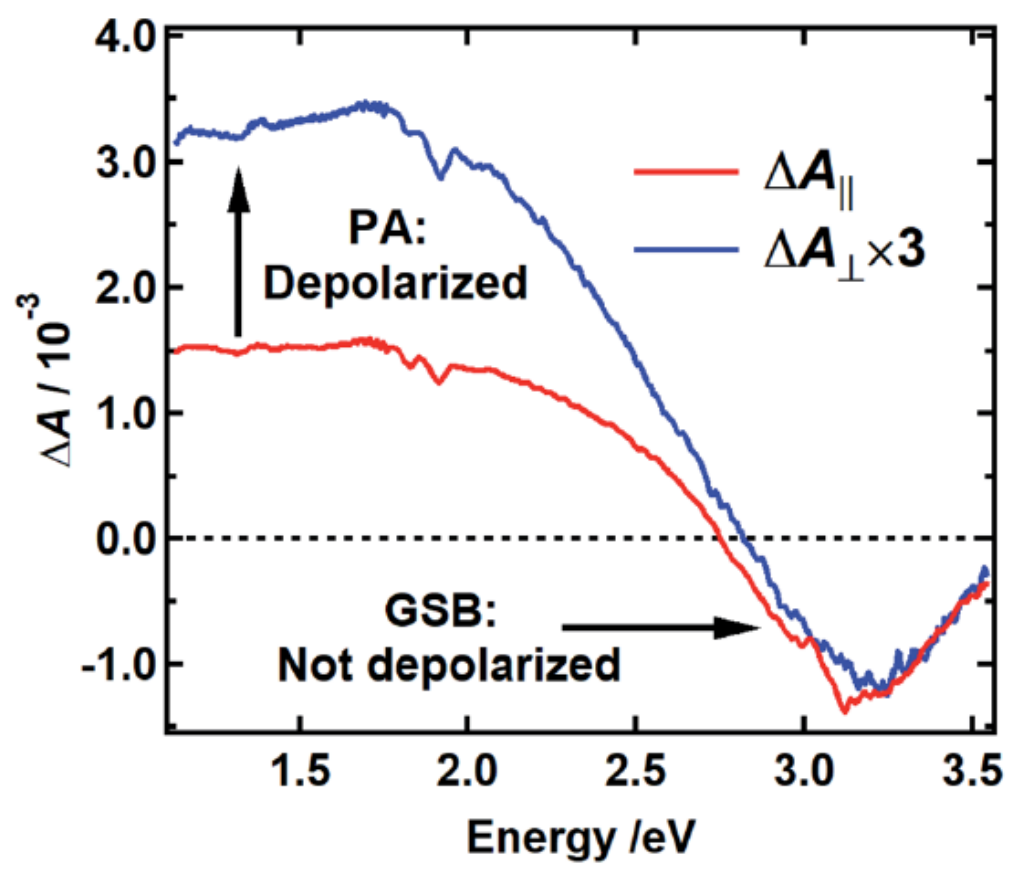

Figure 1.23: Polarization-dependent spectra of the synthetic eumelanin. Reproduced from [92] with permission from the Royal Society of Chemistry.

should be included in any analysis of eumelanin. The polarization anisotropy can as well be an intrinsic property of the fundamental building blocks, and not arise from the CT states formation. Additionally, CT state formation has low oscillator strength [103] which results in a weak PIA band. This was not presented in the study of the CT state complex formation. Hence, further evidence for the CT states recombination mechanism is still needed. 


\section{Research questions}

To this day there is no agreement on how eumelanin dissipates the UV energy with such great efficiency. The understanding of that process would provide us with the biomimetic principles to create optical components (such as windows or filters) and sunscreens.

The chemical and structural disorder complicates studies of the pigment, as well as the ultrafast character of the UV energy dissipation. The research, conducted so far, while creating a clearer understanding of the eumelanin behaviour, did not address the full picture, which would include the study of both eumelanin and its key building blocks. The previous studies of eumelanin and its building locks revealed inconsistencies between them. For example, the role of DHICA in eumelanin energy dissipation was not considered when studying the charge transfer states formation. DHICA building blocks have shown their importance in the ultrafast energy dissipation in eumelanin, and hence they cannot be ruled out of consideration. This raised questions about the validity of the conclusions.

To address the discrepancies in the previously published results we have applied ultrafast spectroscopy methods such as transient absorption and photoluminescence to the synthetic eumelanin and its key building blocks DHI and DHICA. The combination of those methods allows us to focus on radiationless transitions in eumelanin as well as the role of the small building blocks; for example, the photoluminescence studies of DHICA allowed us to perform Förster analysis to describe the role of this building block as photoacid. The study of the building blocks allows us to perform a bottom-up approach of studying the nature behind the effective photoprotection of eumelanin. For that, several questions are addressed in this thesis:

- What are the roles of the key building blocks in energy dissipation?

- How does the oligomerization of the main units affect the energy dissipation rates? 
- Does the disorder of the system play a functional role in the energy dissipation? Are excitations mobile or do they relax locally?

- What is the primary mechanism of the energy dissipation in eumelanin? 
Chapter 2

\section{Experimental approach and methods}

\subsection{Ultrafast optical spectroscopy}

In general, optical spectroscopy is the study of light-matter interactions related to the absorption, emission or scattering of the light by the molecules under the study [71]. The processes happening on the atomic scales are ultrafast, i.e. happening at the very short timescales - from picoseconds (ps) which is $10^{-12}$ of one second to femtoseconds (fs) which is $10^{-15}$ of one second.

To investigate the processes involving the motion of atoms or electrons following the light incidence we have to use lasers with ultrashort pulse lengths. Modern lasers can produce short pulses around $100 \mathrm{fs}$ length which allows us to investigate the dynamics of the excited state of a molecule. The laser pulses are normally shorter than the investigated processes which allow us to study them in real time.

The lasers emit coherent, monochromatic and collimated light beams of high intensity. By reaching high power, the laser light can induce the nonlinear effects when passing through transparent media, which can produce light of different frequencies. Therefore, the laser pulses can be tunable to produce the desired wavelength suitable for activating the electronic transitions in the studied system.

\subsection{Examples of the generated femtosecond pulses}

\subsubsection{Nonlinear optics}

The light propagation through transparent media, or nonlinear crystal, is described with the help of nonlinear optics laws. Nonlinear optics characterizes the changes in the properties of a material in the presence of light. To modify the optical properties of a system the laser light is commonly used since it is sufficiently intense to alter the 
material. The nonlinearity of the system consists of the observed nonlinear manner of the material's response [17].

To describe the optical nonlinearity, we consider the polarization of the material $\tilde{P}(t)$ and its dependence on the strength of an applied optical field $(\tilde{E}(t))$. In the case of linear optics we would observe the linear dependence of the polarization on the electric field strength:

$$
\tilde{P}(t)=\epsilon_{0} \chi^{(1)} \tilde{E}(t),
$$

where $\chi^{(1)}$ is the linear susceptibility, and $\epsilon_{0}$ is the permittivity of free space [17].

When the incident optical field is of sufficient intensity, higher orders of polarization arise. The polarization of the material under the exposure of the electric field can then be described as [17]

$$
\tilde{P}(t)=\epsilon_{0}\left[\chi^{(1)} \tilde{E}(t)+\chi^{(2)} \tilde{E}^{2}(t)+\chi^{(3)} \tilde{E}^{3}(t)+\ldots\right],
$$

where $\chi^{(i)}$ is the $\mathrm{i}$-th order optical susceptibility. The first term is the linear response of the material. With the higher electric field strengths, the higher terms will also be making a contribution to the resulting polarization [53].

\subsubsection{Second harmonics generation}

The second harmonics generation (SHG) is the easiest example of nonlinear interaction in a material (Figure 2.1). When the laser beam whose electric field strength is represented as

$$
\tilde{E}(t)=E e^{-i \omega t}+\text { c.c. }
$$

is incident upon a crystal with the non-zero second order susceptibility, the nonlinear polarization would be

$$
\tilde{P}^{(2)}=\chi^{(2)} \tilde{E}^{2}(t)=2 \epsilon_{0} \chi^{(2)} E E^{*}+\left(\epsilon_{0} \chi^{(2)} E^{2} e^{-2 \omega t}\right)+\text { c.c. }
$$

The second order polarization has a contribution of the frequency $2 \omega$. This contribution leads to the generation of the second harmonic frequency [17]. 

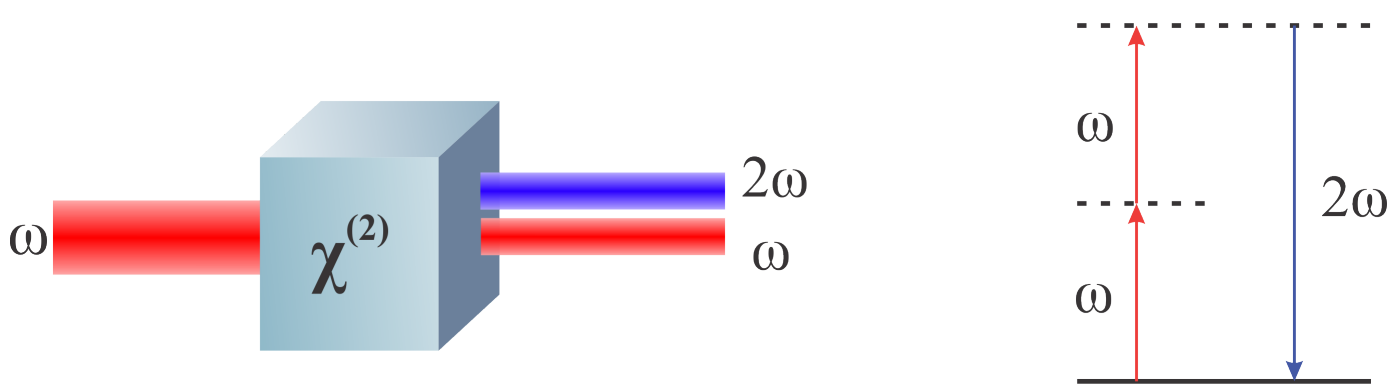

Figure 2.1: Geometry of the second harmonic generation and the energy level diagram of the process. Adapted from [17].

The SHG process can be described as a special case of the sum frequency generation process, when two photons of the frequency $\omega$ are merged through the atomic virtual levels to create a photon of the frequency $2 \omega$, as illustrated in the right panel of the Figure 2.1. For the SHG process to be efficient, the phase matching condition for the wave vectors $\vec{k}$ should be satisfied [53]

$$
\vec{k}_{1}+\vec{k}_{2}=\vec{k}_{3}
$$

Or, in the case of the SHG this equation can be simplified. Here, the pump beam has index 1 and second harmonic has index 2 :

$$
2 \vec{k}_{1}=\vec{k}_{2} \Rightarrow 2 \frac{n_{1} \omega}{c}=\frac{n_{2} 2 \omega}{c} \Rightarrow n_{1}=n_{2}
$$

In other words, the refractive index of the material should be the same for the fundamental and the generated second harmonic. The solution for that is to use birefringent materials like Potassium Dihydrogen Phosphate (KDP) or Beta Barium Borate (BBO). The refractive index of a birefringent material depends on the polarization of the incident light. In the crystals like those, there can be two types of waves - ordinary and extraordinary. For the ordinary wave, the crystal acts like an isotropic body, i.e. the refractive index $\left(n=\sqrt{\epsilon_{\perp}}\right.$ where $\epsilon$ is the dielectric constant) will be angle-independent. The polarization direction of the ordinary wave is perpendicular to the optical axis. For the extraordinary wave, the refractive index depends on the angle $\theta$ between the wave vector and the optical axis [98] 


$$
\frac{1}{n(\theta)^{2}}=\frac{\sin ^{2} \theta}{\epsilon_{\|}}+\frac{\cos ^{2} \theta}{\epsilon_{\perp}}
$$

So that the wave velocity will be

$$
v=\frac{c}{\sqrt{\epsilon}}=c \sqrt{\frac{\sin ^{2} \theta}{\epsilon_{\|}}+\frac{\cos ^{2} \theta}{\epsilon_{\perp}}}
$$

Now using the equations (2.7) and (2.8) we can find the refractive indices for the ordinary and the extraordinary waves:

$$
n_{\perp}=\frac{c}{v_{\perp}}, \quad n_{\|}=\frac{c}{v_{\|}}=\left(\frac{\sin ^{2} \theta}{\epsilon_{\|}}+\frac{\cos ^{2} \theta}{\epsilon_{\perp}}\right)^{-1 / 2}
$$

Here $n_{o} \equiv n_{\perp}$ is the angle-independent ordinary refractive index of the crystal. When the extraordinary wave propagates perpendicularly to the optical axis, $n_{\|}=$ $\sqrt{\epsilon_{\|}} \equiv n_{e}$. The constant $n_{e}$ is the extraordinary refractive index of the crystal (not to confuse with the $n_{\|}=n_{e}(\theta)$ which is the angle-dependent function, representing the refractive index of the extraordinary wave). Now we can derive the $n_{e}(\theta)[26,160]$

$$
n_{e}(\theta)=\frac{n_{e} n_{o}}{\sqrt{n_{o}^{2} \sin ^{2}(\theta)+n_{e}^{2} \cos ^{2}(\theta)}}
$$

By choosing the correct crystal cutting angle $\theta$, we can conserve the phase matching condition (2.6).

\subsubsection{Third harmonics generation setup build to expand the cur- rent setup}

UV radiation is of particular interest in studies of eumelanin and its building blocks as this radiation causes hazardous effects in the human body. The optical parametric amplifier (which will be described later in this chapter), used in our laboratory to convert the fundamental harmonic into different wavelengths, allows us to generate pulses in the UV range. However, due to the long pathlength of the pump beam, 
the generated pulse would be extremely weak at the sample position and cannot be used to excite the sample. To bypass this problem, the UV pump pulses should be generated as close to the sample as possible. For that, we needed to build the setup generating the third harmonic.

We designed the frequency tippler, based on the materials with the strong nonlinearity and high damage threshold, like KDP or BBO, that have a high nonlinear response. The nonlinear calculations discussed in the previous sections were used to chose the correct crystals for the built setup. We calculated the crystals' specifications, like thickness and cutting angle, for the used laser system, and after choosing the correct setup elements, built the third harmonic generation setup.

Third harmonic generation (THG) is a sum frequency generation process $\omega_{1}+$ $\omega_{2}=\omega_{3}$ with $\omega_{1}=\omega, \omega_{2}=2 \omega$, and $\omega_{3}=3 \omega$ (Figure 2.2). Under the oo-e type 1 phase matching conditions (when the fundamental and the second harmonic are the ordinary $(o)$ waves and the generated third harmonic is an extraordinary $(e)$ wave), the phase matching condition will look like

$$
n_{1}+2 n_{2}=3 n_{3}
$$

Another possibility of the THG is to use the oe-e type 2 phase matching condition when the second harmonic is an extraordinary $(e)$ wave. If that is the case for the SHG, the phase matching condition will become

$$
n_{1}+n_{2}=2 n_{3}
$$

where $n_{1}$ and $n_{2}$ are the refractive indices for the fundamental in the ordinary and the extraordinary polarization and $n_{3}$ is the refractive index for the second harmonic in the extraordinary polarization.

The type 1 phase matching conditions are more favourable for the two-step process of the THG [12]. For that, the polarization of the fundamental and second harmonics should be in the same direction, but after SHG the polarizations of fundamental and second harmonic are perpendicular to each other.

To correct the polarization and satisfy the phase matching conditions, it is useful to insert a half wave plate between the two nonlinear crystals. For the setup built for 


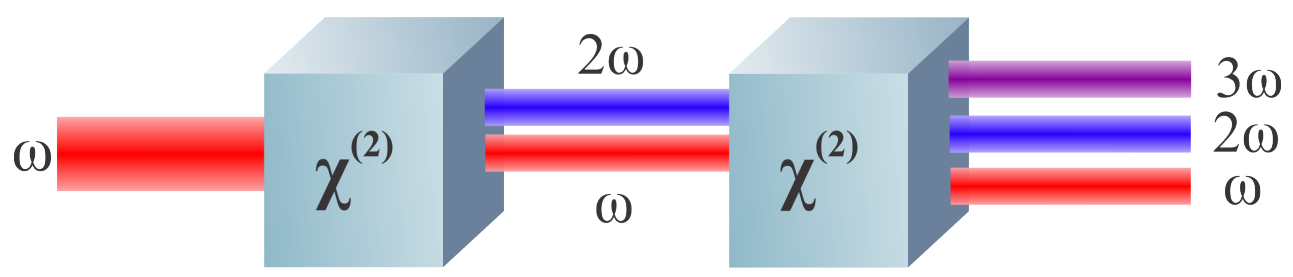

Figure 2.2: Third harmonic generation process.

the eumelanin experiment, we have chosen the dual zero-order waveplate (Eksma Optics). It changes only the polarization of the fundamental, and acting like a full waveplate for the second harmonic, leaving its polarization unaltered. The setup is shown in Figure 2.3.

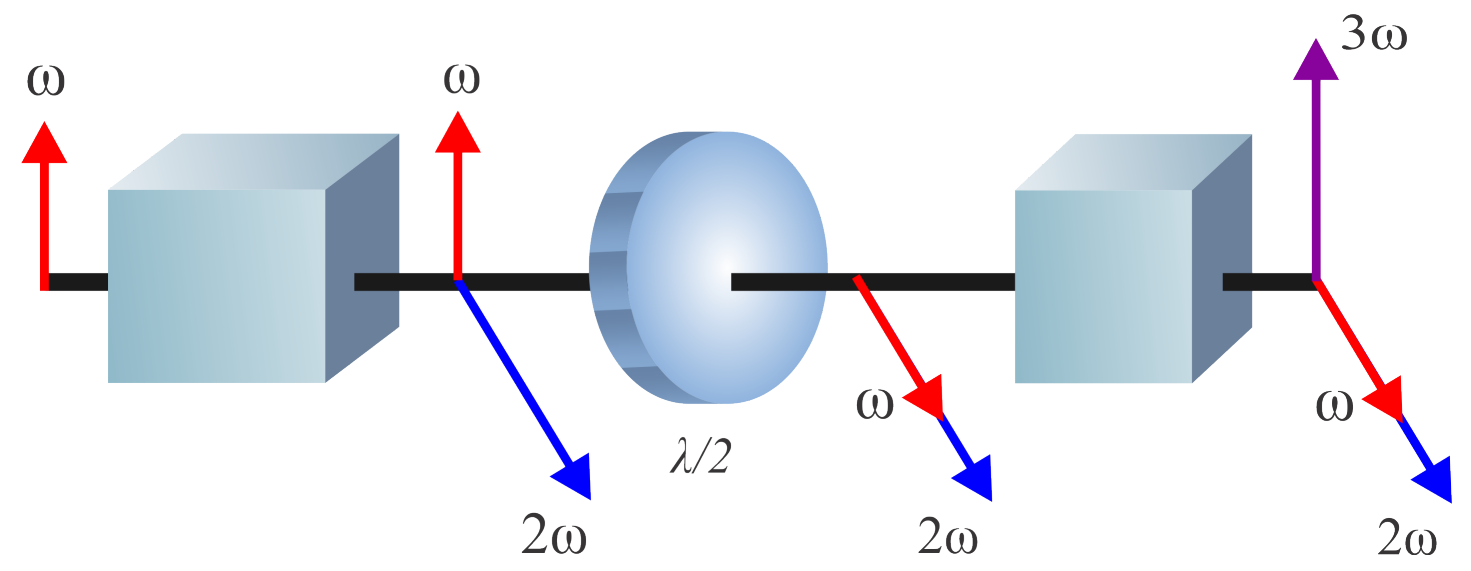

Figure 2.3: Two-step process of THG. The time delays from the crystals are not indicated.

This setup, however, while working perfectly with long pulses, has major flaws when used with the femtosecond pulses. The problem is that the group velocity difference between the fundamental and the second harmonics in the half wave plate introduces a delay longer than the pulse duration [53].

To correct the time delay, resulting from introducing the waveplate, we have used a tunable group velocity compensation plate. It is a birefringent crystal that is cut with the optical axis oriented at a suitable angle. BBO and Calcite are commonly used; both have a low value of the refractive index for the extraordinary wave. The layout for the setup used for THG is shown in Figure 2.4.

Under type 1 phase matching conditions, polarization directions of the funda- 


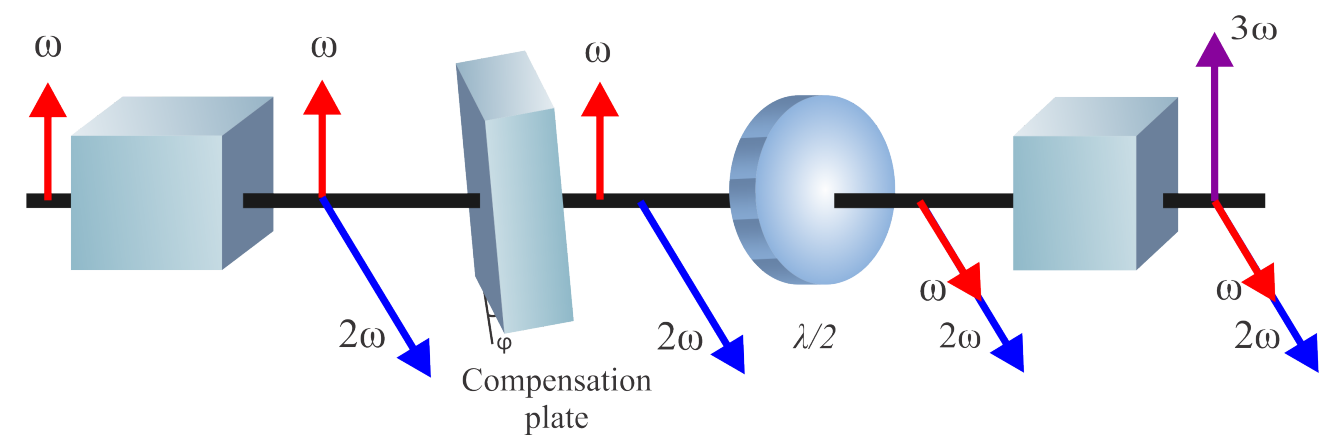

Figure 2.4: Third harmonics generation setup.

mental and second harmonics are perpendicular to each other. If the compensation plate is oriented the way that the optical axis is perpendicular to the polarization direction of the fundamental, the second harmonic will become an extraordinary wave and will have a lower refractive index than the fundamental. Then the time delay can be adjusted by rotating the compensation plate. The compensation plate should be put before the waveplate since it only works when two harmonics have different polarizations. To find the right compensation plate we need to know the time delays introduced by each of the components of the setup. For that, we need to know the group velocities of the propagating waves. The general formula for the velocity is

$$
v=\frac{\omega}{k}
$$

When the component waves that are travelling together have different velocities, they create internal oscillations in the envelope. The speed of these internal oscillations is called the phase velocity $\left(v_{p}\right)$. The velocity of the wave envelope is called the group velocity $\left(v_{g}\right)$

$$
\begin{aligned}
& v_{p}=\frac{\omega}{k} \\
& v_{g}=\frac{d \omega}{d k}
\end{aligned}
$$

Now we can substitute the equation (2.14) into the equation (2.15) [132] 


$$
v_{g}=\frac{d}{d k}\left(k v_{p}\right)=v_{p}+k\left(\frac{d v_{p}}{d k}\right)
$$

In the non-dispersive media (like a vacuum), the velocity of a wave does not depend on the wavelength, so the phase and group velocities will be the same. In the dispersive media $v_{p}=c / n(\lambda)$ (or $v_{p}=c / n(k)$ ) [132]

$$
\frac{d v_{p}}{d k}=\frac{d}{d k}\left(\frac{c}{n}\right)=-\frac{c}{n^{2}}\left(\frac{d n}{d k}\right)
$$

When we substitute this value into equation (2.16), we get the relationship between phase and group velocities

$$
v_{g}=v_{p}\left[1-\frac{k}{n}\left(\frac{d n}{d k}\right)\right]
$$

Now we reformulate that using the fact that $k=2 \pi / \lambda$

$$
v_{g}=v_{p}\left[1+\frac{\lambda}{n}\left(\frac{d n}{d \lambda}\right)\right]
$$

To find the group velocity for each of the propagating waves, we need to substitute the suitable refractive indices expressions. The ordinary and the extraordinary refractive indices can be found using the Sellmeier relations [168] showing the refractive index dependence on the wavelength. These equations are used to determine the dispersion of light while it propagates inside the medium. For the THG setup, we used the BBO for the harmonics generation and as the compensation plate and the fused quartz waveplate. The waveplate gives the largest contribution to the total time delay, and its dispersion formula is [104]

$$
n_{o / e}^{2}-1=\frac{A_{o / e} \lambda^{2}}{\lambda^{2}-B_{o / e}}+\frac{C_{o / e} \lambda^{2}}{\lambda^{2}-D_{o / e}}+\frac{E_{o / e} \lambda^{2}}{\lambda^{2}-F_{o / e}}
$$

For the BBO the expression will be different [49]

$$
n_{o / e}^{2}=A_{o / e}+\frac{B_{o / e}}{\lambda^{2}-C_{o / e}}-D_{o / e} \lambda^{2}
$$

The coefficients for the ordinary and extraordinary waves for each of the materials can be found on the manufacturer's website or manuals. 
Now we need to calculate the propagation length for each wave. It was done [99] by solving Maxwell's equations. The waves propagation in a birefringent medium is shown in Figure 2.5. To derive the expressions for the pathlengths for each wave we should look at the geometry of the propagation more closely.

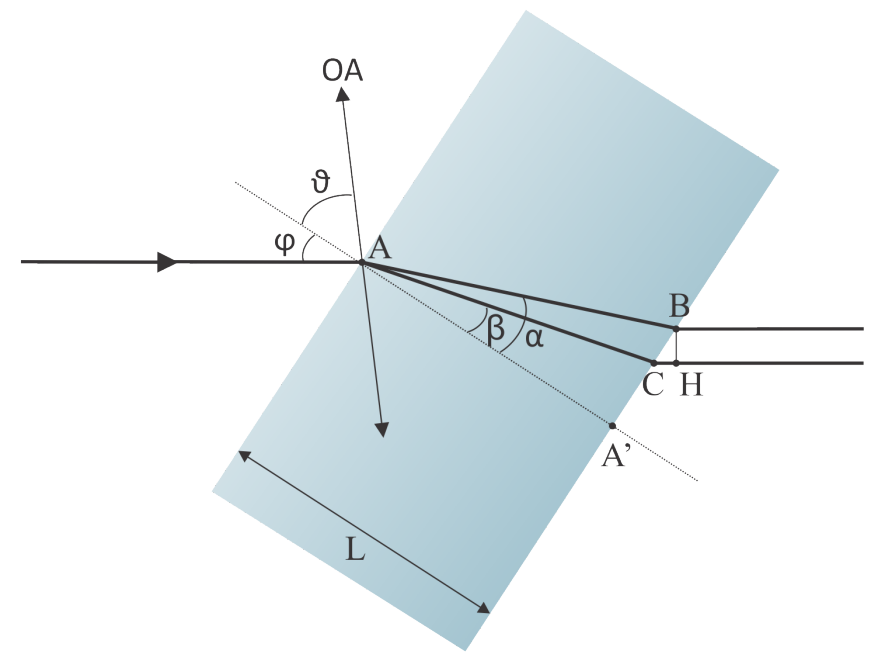

Figure 2.5: Different paths for ordinary $(A C)$ and extraordinary $(A B)$ waves in the birefringent crystal.

As illustrated, $\theta$ is the angle between the surface normal and the optical axis and $\phi$ is the angle of the incidence, also called tuning angle. At the point $A$ the beam is separated into the ordinary and extraordinary waves.

The direction of propagation for extraordinary wave $[53,99]$ is given by

$$
\tan \alpha=\frac{Q_{e} \sin \theta \sin \varphi-\left(\epsilon_{o}-Q_{e}^{2}\right) \cos \theta}{Q_{o}^{2} \sin \theta-Q_{e} \sin \theta \sin \varphi}
$$

And the direction of the $\vec{k}$-vector of the extraordinary wave is

$$
\tan \alpha_{k}=\frac{\sin \varphi}{Q_{e}}
$$

Here $\epsilon$ is the dielectric constant, $\Delta \epsilon=\epsilon_{e}-\epsilon_{0}$, and the constants $D$ and $Q$ are defined as $[53,99]$ 


$$
\begin{aligned}
D & =\left[\epsilon_{o} \epsilon_{e}\left(\epsilon_{o}+\Delta \epsilon \cos ^{2} \theta-\sin ^{2} \varphi\right)\right]^{1 / 2}, \\
Q_{o}^{2} & =\epsilon_{o}-\sin ^{2} \varphi, \\
Q_{e} & =\frac{D-\frac{\Delta \epsilon}{2} \sin 2 \theta \sin \varphi}{\epsilon_{o}+\Delta \epsilon \cos ^{2} \theta}
\end{aligned}
$$

And the direction of the propagation of the ordinary wave is

$$
\sin \beta=\frac{\sin \varphi}{n_{0}}
$$

After propagating through the crystal, two waves are propagating parallel to each other through the air from the point $B$. In that case, the path for the ordinary wave would be $A C$ inside the crystal and $C H$ in the air. The extraordinary wave only passes through the crystal $(A B)$.

Using the basic trigonometry, we extract the distances $A C$ and then $C H$ which is the difference of $A^{\prime} B-A^{\prime} C$ :

$$
\begin{aligned}
A C & =\frac{L}{\cos \beta} \\
C H & =L(\tan \alpha-\tan \beta) \sin \varphi
\end{aligned}
$$

For the extraordinary wave, we cannot just use the $A B$ expression found with the trigonometric calculations as its $\vec{k}$-vector is in another direction. Instead, we use a projection of $A B$ onto the $\vec{k}$-vector

$$
A B_{k}=\frac{L}{\cos \alpha} \cos \left(\alpha-\alpha_{k}\right)
$$

The expression for the refraction index for the $\vec{k}$-vector of the extraordinary wave will also be transformed $[53,99]$ as

$$
n_{k}=\frac{n_{e} n_{o}}{\sqrt{n_{o}^{2} \sin ^{2}\left(\alpha_{k}+\theta\right)+n_{e}^{2} \cos ^{2}\left(\alpha_{k}+\theta\right)}}
$$

So the time delay in that case can be calculated as 


$$
\Delta t=t_{o}-t_{e}=\frac{A C}{v_{g}^{o}}+\frac{C H}{c}-\frac{A B_{k}}{v_{g}^{e}}
$$

The first component represents the ordinary wave propagation in the crystal, the second one is the ordinary wave propagation in air and the last one is the extraordinary wave propagation in the crystal.

The time delay can be controlled by rotating the compensation plate and it is calculated according to the type of crystals used, their cutting angle and the thickness. The tuning curve for the $6 \mathrm{~mm}$ thick BBO compensation plate is shown in Figure 2.6.

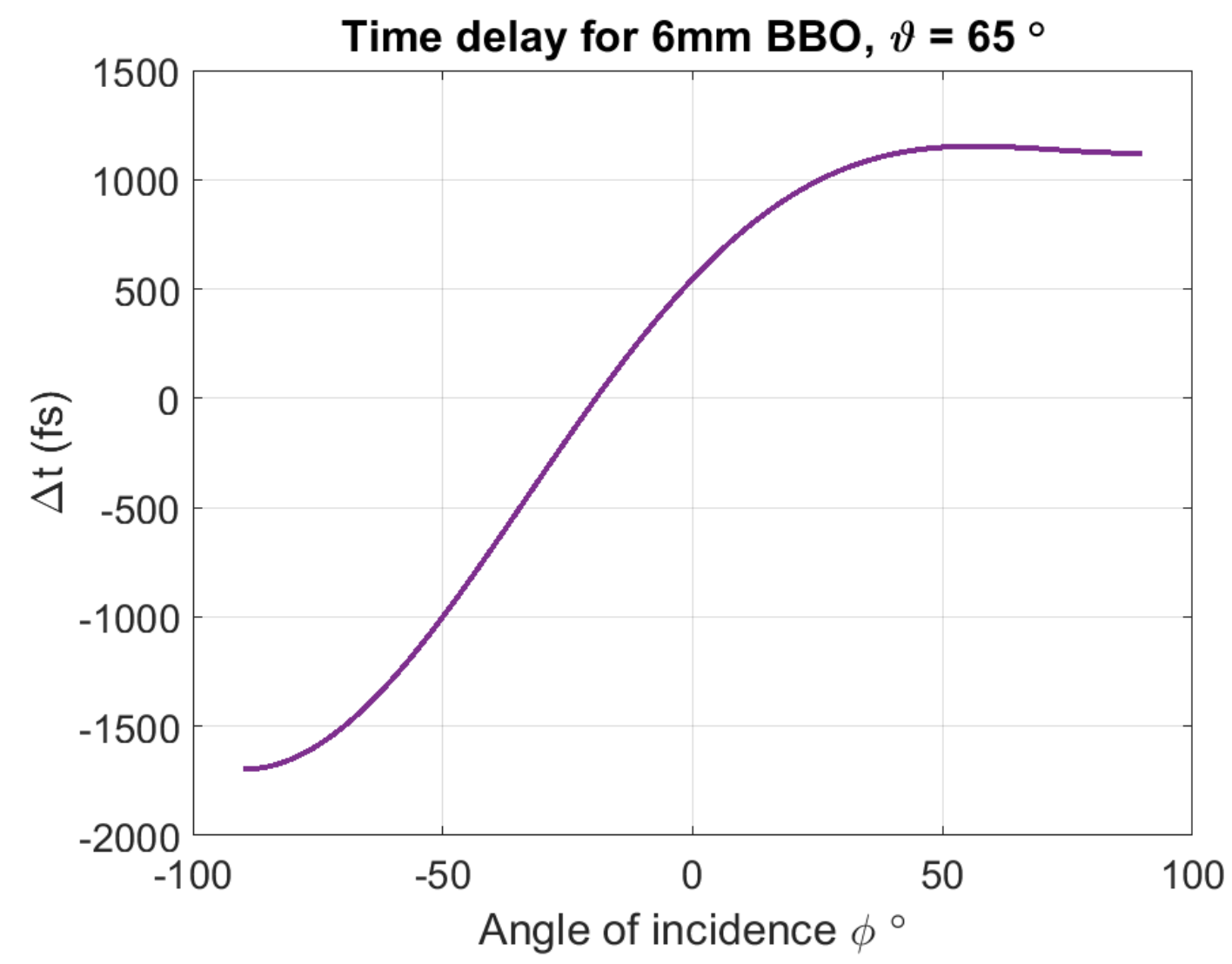

Figure 2.6: Tuning curve for a $B B O$ compensation plate.

The crystals used in the resulting setup are listed in Table 2.1. The desired time delay was calculated at 611 fs. As we can see from the tuning curve in Figure 2.6, 
Table 2.1: List of crystals for the third harmonic generation.

\begin{tabular}{||c|c|c|c|c||}
\hline & SHG & Compensation plate & $\lambda / 2$ waveplate & THG \\
\hline \hline Crystal type & BBO & BBO & Quartz & BBO \\
\hline Thickness & $0.5 \mathrm{~mm}$ & $6 \mathrm{~mm}$ & $2 \mathrm{~mm}$ & $0.5 \mathrm{~mm}$ \\
\hline Cutting angle & $29.2^{\circ}$ & $65^{\circ}$ & & $44.3^{\circ}$ \\
\hline
\end{tabular}

we only need to rotate the compensation plate at roughly $3^{\circ}$. That allows avoidance of a large angle of incidence, which can result in the loss of efficiency.

The generation of the third harmonic is just the sum frequency generation process using the mixture of the fundamental and the second harmonics. The crystal for the THG is usually relatively thin because the fundamental and the second harmonic propagate with different velocities and will only overlap over a short distance. For the effective harmonic generation, the fundamental and the second harmonics should overlap inside the crystal at this limited distance, so there is no need to use a thick crystal for the THG. After the generation of the third harmonic, the contribution of the fundamental and the second harmonics are removed with the help of harmonic separators. To ensure the purity of the generated third harmonic signal, a wire grid polarizer is inserted after the harmonic separators. Since the polarization of the third harmonic is perpendicular to the polarizations of the fundamental and the second harmonic, by setting the polarizer's angle to a correct value, we ensure that only the third harmonic will pass through. The residuals of the second harmonics and the fundamental, while noticeable at the spectrometer reading, showed in Figure 2.7, are negligible and do not affect the sample excitation.

The output efficiency of the built THG setup is not very high - at the fundamental's power $600 \mathrm{~mW}$ the third harmonic's power was $6 \mathrm{~mW}$, which gives only $1 \%$ efficiency, while it was claimed [53] to be able to reach $8 \%$. The reason for such a low output originates in the low beam quality. The laser's output beam diameter is $14 \mathrm{~mm}$, while the input aperture of the used nonlinear crystals was only $\sim 6 \mathrm{~mm}$. Therefore, to ensure the full beam propagation through the crystals, the beam size should be reduced drastically using the telescope. The telescope consists of one con- 


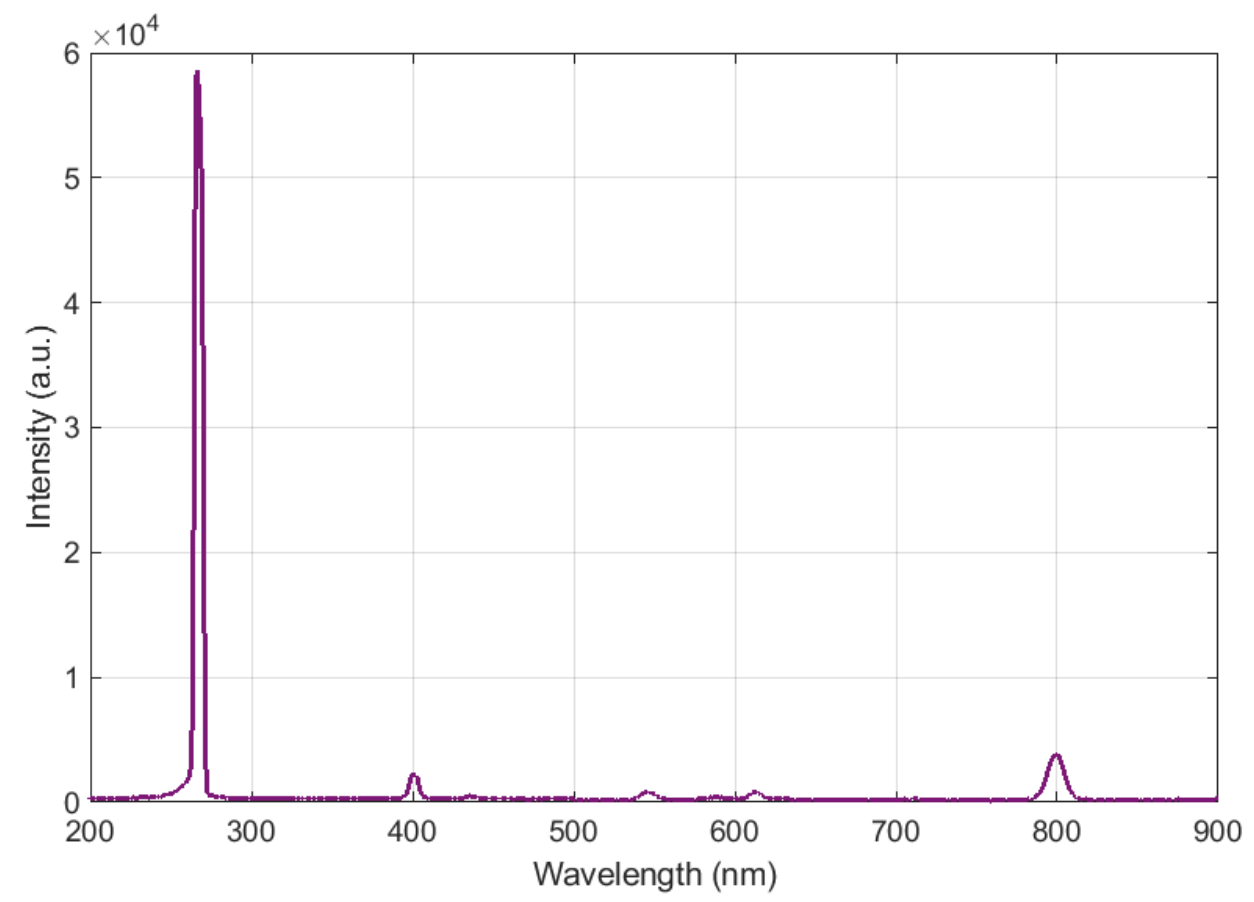

Figure 2.7: Third harmonics signal from the built setup.

cave and one convex lens, set up to the distance between them equal to the sum of their focal lengths. The strong decreasing of the beam size might introduce astigmatism that affects the beam's quality and therefore the output efficiency of the third harmonic generator. However, the output power of $6 \mathrm{~mW}$ was sufficient to conduct the experiments so it did not affect the measurements.

\subsubsection{Supercontinuum}

To probe the excited state, we use broadband pulses also called white light or supercontinuum. This phenomenon happens when intense focused monochromatic light passes through the transparent medium. The supercontinuum generation in transparent bulk media dates back to 1970 when Alfano and Shapiro [5] first reported white light generation in the borosilicate glass by self-focusing of intense picosecond pulses in the sample. This was followed by the observation of spectral broadening in various crystals and glasses [6]. The broadband supercontinuum has 
been used in various ultrafast spectroscopy techniques [24, 44, 63, 178].

The supercontinuum generation happens as a result of a process called femtosecond filamentation - the nonlinear propagation and the following spectral broadening of the ultrashort pulses in the liquids, solids and gases $[39,48,85,120,182]$. The light-matter interaction processes resulting in the supercontinuum generation involve self-focusing, self-phase modulation, and multi-photon absorption [47]. The most important process in the supercontinuum generation in the transparent bulk media is the optical Kerr effect, describing the intensity-dependent refractive index of the medium:

$$
n(t)=n_{0}+n_{2} I(t)
$$

where $n_{0}$ and $n_{2}$ are the linear and non-linear refractive indices, respectively, and $I$ is the intensity. During the propagation of the laser beam through the material, the induced change of the refractive index is proportional to the intensity and therefore the beam is exposed to a higher refractive index in the center and lower at the edges. The material acts like a lens which then causes the beam to self focus inside the material [23]. The balance between self-focusing and defocusing is called filamentation so the beam propagates through the media without diffraction. Concurrently, the refractive index induces changes in the phase of the pulse in the time domain, so the light modifies the material it is passing through. The amount by which the refractive index of the material increases depends on the intensity of the propagating light. The change in the refractive index induces a change of the spatial phase of the wave. In the time domain, the time dependence of the refractive index produces a nonlinear change in the phase of the pulse [48]. That nonlinear phase shift is accumulated during the pulse propagation. In the front half of the wave, the refracting index is rising in time, which reduces the frequency of the wave, while in the rear half the refractive index is falling in time, causing the increase of the wave's frequency. This produces the frequency change that results in the generation of new frequencies. This process is called self-phase modulation and it results in the spectral broadening of the pulse [4].

For the observation of the excited state of eumelanin, we have used two dif- 


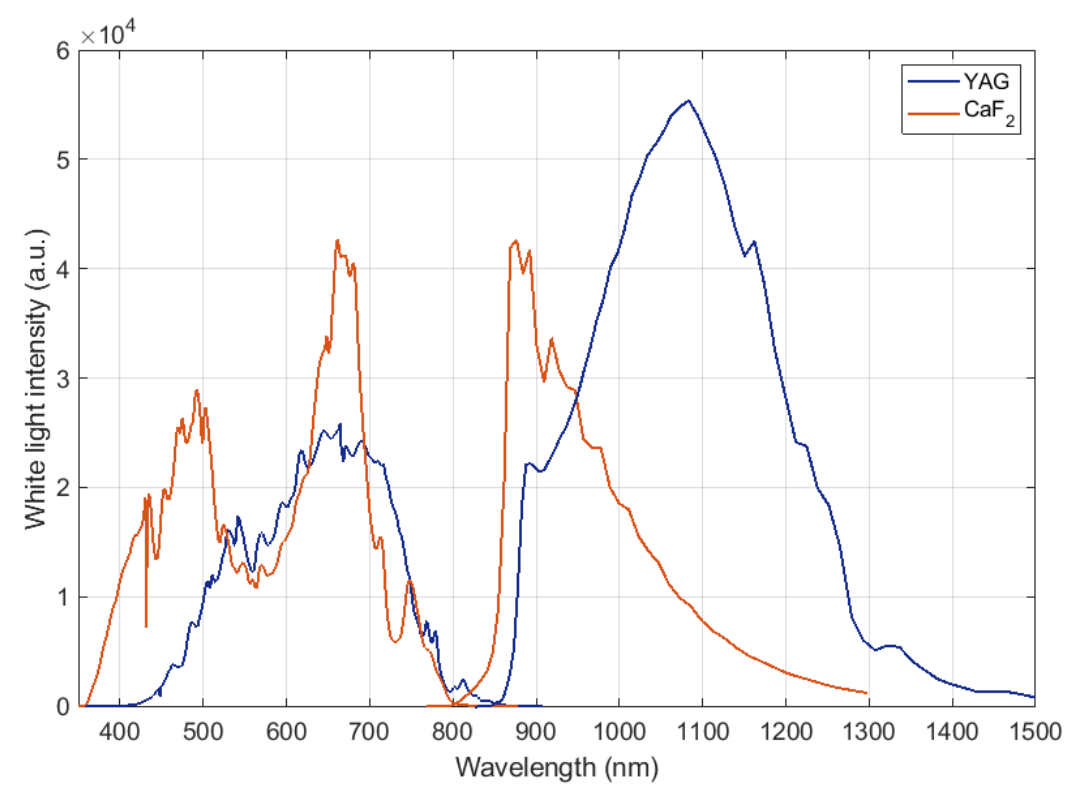

Figure 2.8: White light continuum, produced by $\mathrm{CaF}_{2}$ and $Y A G$ crystals. The drop of intensity around $800-850 \mathrm{~nm}$ region is coming form blocking the $800 \mathrm{~nm}$ contribution and merging visible and IR cameras at $850 \mathrm{~nm}$.

ferent crystals to generate white light. A calcium fluoride crystal $\left(\mathrm{CaF}_{2}\right)$ was used predominantly due to its capacity to reach the UV region of the spectra [42], which is the region of interest for eumelanin studies. An Yttrium-Aluminum Garnet (YAG) nonlinear crystal was also used to study the IR region of the spectra. Even though the YAG crystal has the smaller bandgap than the $\mathrm{CaF}_{2}$ crystal $(5.5 \mathrm{eV}$ vs $9.2 \mathrm{eV}$ respectively), YAG has a stronger signal in the IR region [18] and is better suited for measurements in the Vis-IR study of the pigment behaviour. The spectra of the used $\mathrm{CaF}_{2}$ and YAG crystals' supercontinuum are shown in Figure 2.8.

\subsubsection{Optical parametric amplification}

The pump pulses are monochromatic to selectively trigger a particular ground state - excited state transition in the sample. To generate the different wavelengths for the sample excitation we use an optical parametric amplifier (OPA) [27] TOPAS-C. This two-stage parametric amplifier uses the fundamental $800 \mathrm{~nm}$ to generate different 
wavelengths in the broad range $(240-2600 \mathrm{~nm})$ using nonlinear processes such as supercontinuum generation, second harmonic generation, and sum frequency generation. OPA process transfers energy to form a high power, fixed frequency beam to a low power tuned frequency signal [27]. For the system to be efficient, high pump beam intensities are required to launch the nonlinear processes. Therefore, the OPA system is suited for femtosecond laser systems.

The first stage of the OPA process is the generation of the initial signal beam, or seed beam. The white light generation is often used for the seed generation in OPA. For that, the fundamental $800 \mathrm{~nm}$ is directed to the transparent media, such as fused silica or sapphire plate. The generated supercontinuum extends throughout the visible and near-IR spectral range [27]. The other fraction of the fundamental (pump beam) is focused and overlapped with the generated supercontinuum in the preamplification crystal (first stage of amplification). To achieve temporal overlap, the delay line is used to adjust the timing. The generated signal is then directed into the power amplifier (second stage of amplification). After that the $800 \mathrm{~nm}$ fundamental pumps the power amplifier which is then collinearly overlapped in the second nonlinear crystal with the signal beam produced by the first stage of amplification.

By changing the time delay between the generated supercontinuum and the $800 \mathrm{~nm}$ input and adjusting the crystal angle for the optimal phase matching conditions we can tune the wavelengths produced by the first stage. Similarly, for the second stage, after adjusting the second crystal angle and setting the suitable time delay between the signal and the $800 \mathrm{~nm}$ fundamental, we can tune the output wavelength. Using the different frequency mixers and harmonic separators after the TOPAS-C output, the resulting wavelength can be tuned from the UV to IR spectral range.

\subsection{Transient absorption spectroscopy}

Ultrafast broadband TA spectroscopy is an optical pump-probe technique in which the sample is excited by an intense narrowband monochromatic pulse (the pump) and then the excited state dynamics are monitored by the second broadband pulse 
(the probe). An electronic delay stage is used to change the time at which the pump pulse arrives at the sample. That way, we create a delay between the pump and the probe pulses to observe the time-resolved photo-induced changes in the sample transmission. By varying the time delay between those two pulses the information about the excited states can be obtained as a function of the excitation wavelength and the time after excitation. The pump and probe pulses are spatially overlapped in the sample where the size of the probe is focused with the lens to be smaller than the size of the pump. The intensity of the probe is usually weaker compared to the pump to not trigger probe-induced population transfer from the ground state to the excited state. After passing through the sample, the pump pulses are blocked and the probe pulses are spectrally dispersed via silica prism and then collected on the photodiode array [109]. The scheme of the home-built TA spectrometer is shown in Figure 2.9.

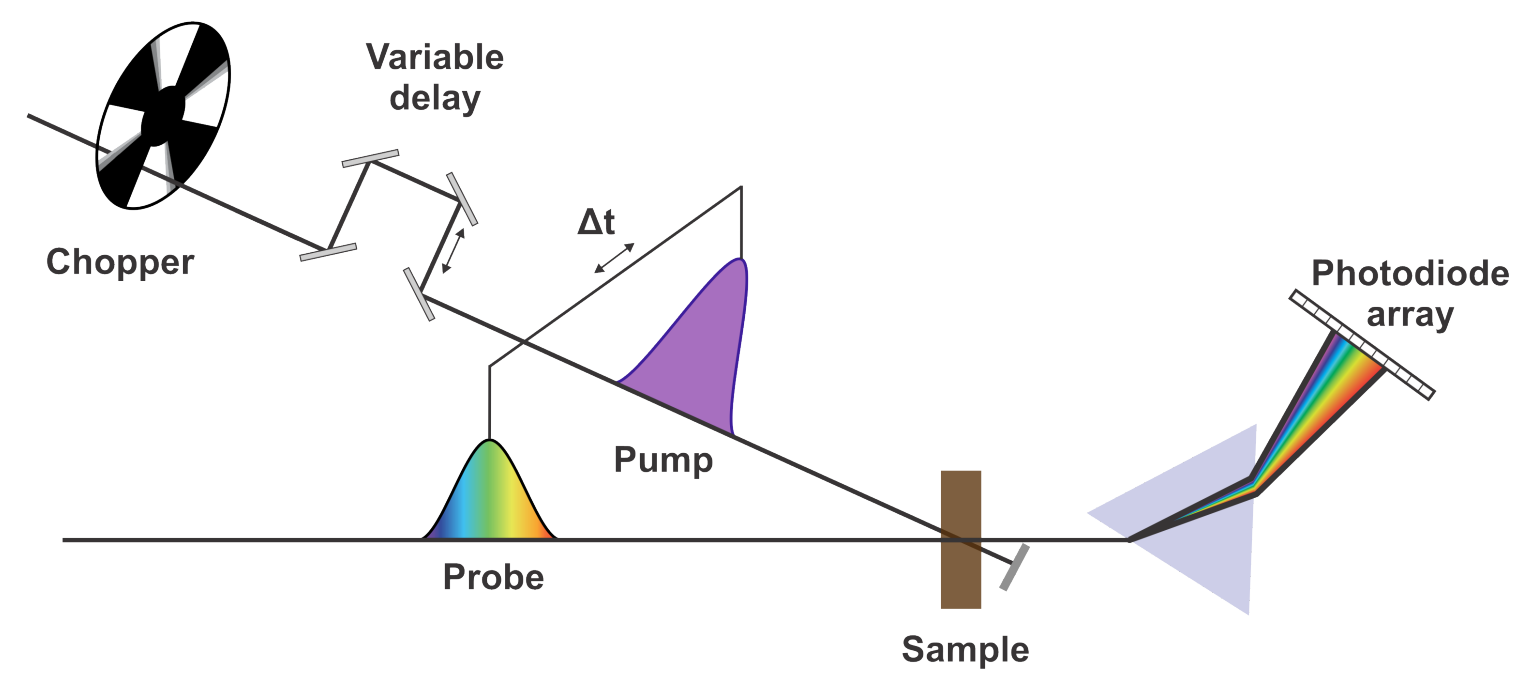

Figure 2.9: Layout for a transient absorption system.

In our setup [13], the laser system is based on the Ti:Sapphire regenerative amplifier (SpitfirePro, Spectra Physics) producing pulses of $100 \mathrm{fs}$ duration, centered at $800 \mathrm{~nm}$ at the repetition rate of $3 \mathrm{kHz}$. The fundamental laser beam is split after the output aperture to generate the pump and probe pulses used in the experiment. The supercontinuum probe pulses were generated with the nonlinear $\mathrm{CaF}_{2}$ crystal like discussed in Section 2.8. The pump pulses were generated with a combination 
of methods like OPA (discussed in Section 2.2.5) for visible and NIR experiments and home-build second and third harmonics generator, discussed in Sections 2.1 and 2.2.3.

\section{Transient absorption signal}

The TA signal is a pump-induced change in the transmission normalized by the transmission of the ground state $(\Delta T / T)$. It can be calculated as

$$
\frac{\Delta T}{T}(\lambda, t)=\frac{T_{o n}(\lambda, t)-T_{o f f}(\lambda)}{T_{o f f}(\lambda)},
$$

where $\Delta T$ is the change in the transmission of the probe, $T_{o n}$ is the transmission of the probe with the pump excitation and $T_{o f f}$ is the transmission of the probe without the pump excitation. It is seen that the two probe pulses (in the pump on and pump off states) are needed to calculate the difference in the transition. We achieve this by blocking every second excitation pulse. For that, we set up the mechanical chopper in the pump beam path. The chopper frequency is set at $\omega / 2$, where $\omega$ is the repetition rate of the fundamental.

The time resolution of the experiment is defined by the instrument response function (IRF). It is given by the duration of the pump and the probe pulses. The wavelength range is determined by the probe pulse spectral width, i.e. it is defined by the choice of the white light generating nonlinear crystal.

A typical TA signal is a complex three-dimensional matrix of data comprising of spectral and kinetics traces. To illustrate the result of one TA measurement the data for the synthetic eumelanin film after $600 \mathrm{~nm}$ excitation is chosen and presented in Figure 2.10. The x-axis represents the different times after the excitation of the sample, while the y-axis shows the wavelength spectral range. For an initial interpretation of TA surface, the "slices" in either time or wavelength domain are taken.

The TA spectra, obtained by taking slices at different time points, shows the spectral behaviour of the sample at different times after the excitation. Spectral traces can contain information about intramolecular transitions which are reflected in the featured peaks. The scheme, illustrating the main transitions, is shown in Figure 2.11. 
There are three effects on the transmitted pulse that can be present in TA spectral slices:

Ground state bleach (GSB). When the pump pulse excites the sample, some of the molecules are transferred from the ground state to an excited state. Thus the concentration of the ground state decreases, increasing the transmission. This results in the positive $\Delta T / T$ signal as shown in Figure 2.10 (b). It should be noted that the GSB signal here overlaps with the strong scattering of the pump beam.

Stimulated emission. This effect occurs when the probe photons stimulate the emission of the molecules from the excited states. This results in additional photons to be collected with the probe pulse giving the positive $\Delta T / T$ signal.

Photo-induced absorption (PIA). This effect happens when the probe photons are absorbed by the molecules in their excited states. This reduces the transmission of the sample resulting in the negative $\Delta T / T$ signal. The PIA part of the spectra, shown in Figure 2.10 (b) contain the peaks around $850 \mathrm{~nm}$ and $1450 \mathrm{~nm}$ that are coming from the artifacts in the white light.

After extracting the spectral traces and identifying the main electronic transitions, we can obtain the kinetics traces, shown in Figure 2.10 (c) by taking slices at different wavelengths, corresponding to the spectral contributions. By choosing the right points we can observe the dynamics of excited states of interest.

The TA data also possess a number of artifacts that should be taken into account during the post-processing or analysing [102] as they might affect the data and result in the wrong interpretation.

Instrument response function. As was briefly mentioned earlier, the IRF is representing the minimal temporal resolution of the system. In the typical TA system, the minimal delay stage step is shorter than the pulse width so at the time zero (the moment of temporal overlap of the pump and probe pulses) the pump cannot instantaneously generate an excited state population. The resulting signal within the IRF region will be overlapped with the Gaussian shape of the pump and the probe pulses. In mathematical terms, the produced kinetics decay shape will be a result of a convolution between the IRF $I(t)$ and the sample response function $R(t)$ [7] : 
(a)

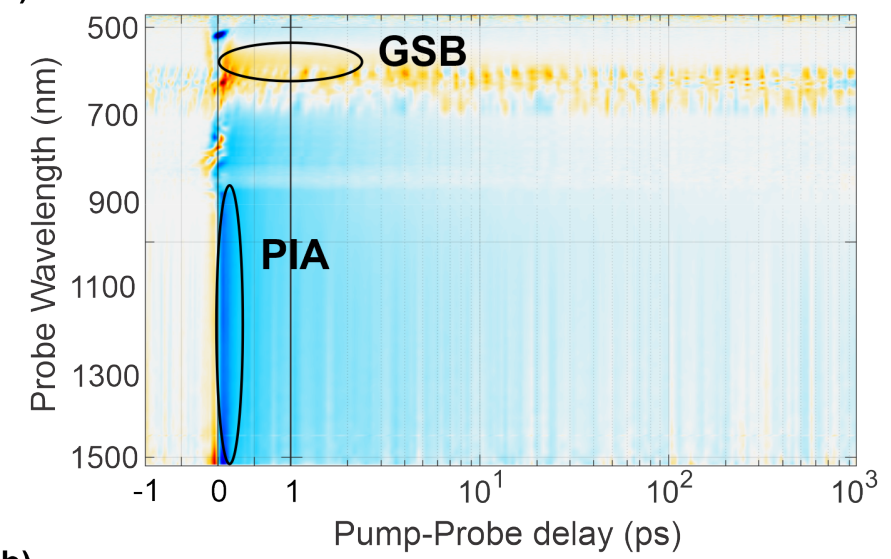

(b)

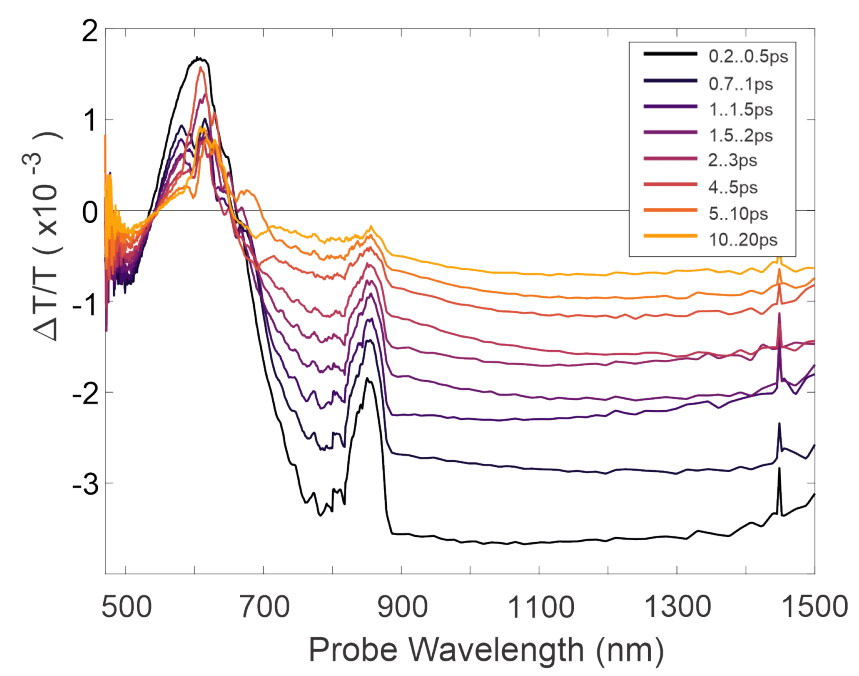

(c)

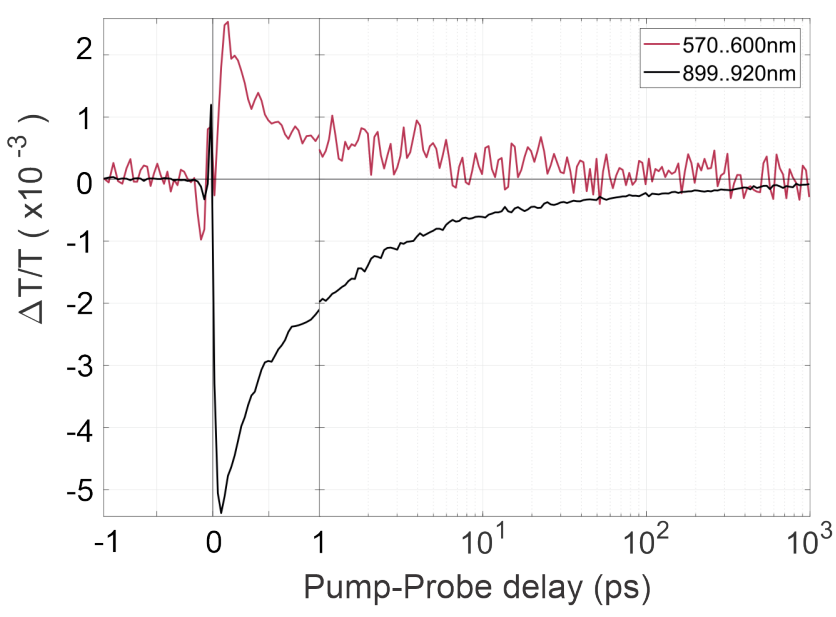

Figure 2.10: Example of TA data for eumelanin: surface (a), spectral traces (b) and kinetics traces (c). 


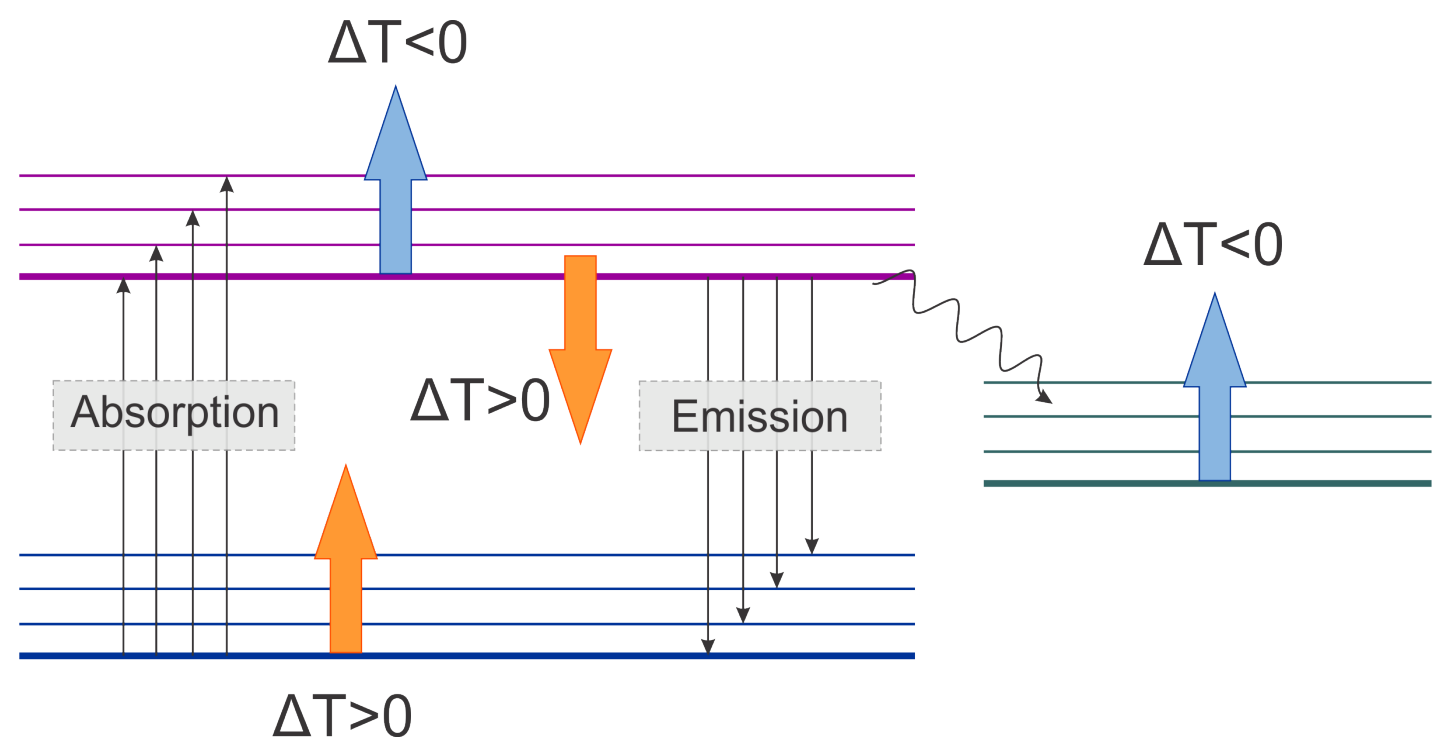

Figure 2.11: The types of signals, contributing in the TA spectra. Adapted from [152].

$$
I(t) \circledast R(t)=\int_{0}^{+\infty} I\left(t^{\prime}\right) \times R\left(t-t^{\prime}\right) d t=\Delta T(t) .
$$

While the IRF shape is postulated to be a Gaussian [185], the sample response function (the decay kinetics) is assumed to be a result of a number $n$ of competing dissipation pathways. Each pathway is individually modelled by a decaying exponential, so our total model is given by

$$
\frac{\Delta T}{T}=\sum_{i=1}^{n} T_{i} \exp \left(-\frac{t}{t_{i}}\right)
$$

where $T_{i}$ is a pre-exponential factor, and $\tau_{i}$ is the mean lifetime for each dissipation pathway.

Chirp. Group velocity dispersion, denoting the wavelength-dependent phase velocity in the transparent media, is responsible for temporal broadening of the supercontinuum after the fundamental propagates through a crystal with Kerr nonlinearity $[23,89]$. When the broad continuum passes through the transparent medium, it experiences a wavelength-dependent refractive index so that each wavelength will be propagating with a different velocity. As a result, we will observe the temporal spreading within the pulse - the chirp. While propagating through a normally 

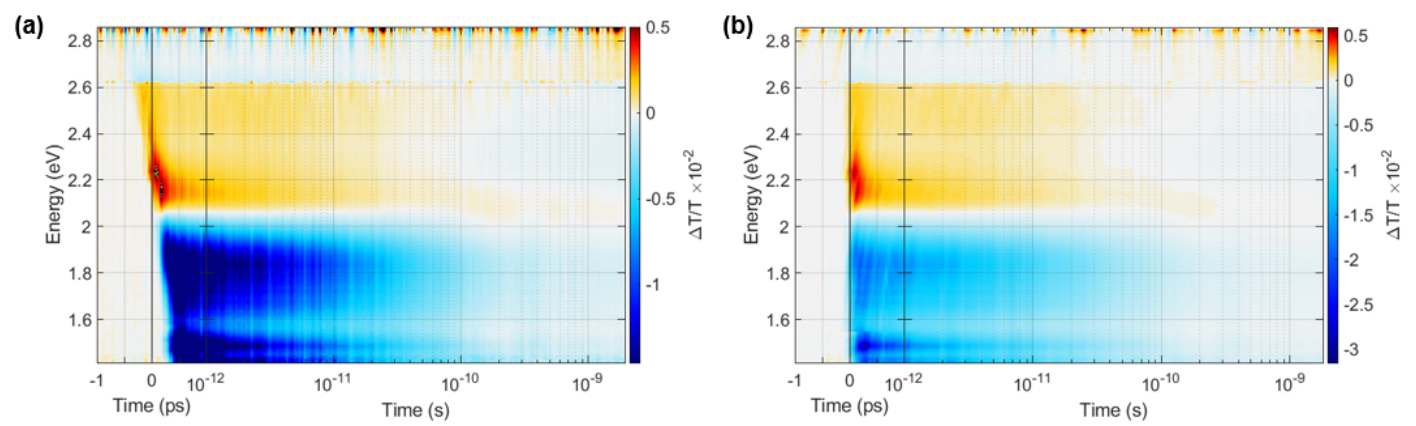

Figure 2.12: Example of the chirp correction in the TA data of the neat Y6 material: TA surface before (a) and after (b) chirp correction.

dispersive media, the pulses will become positively chirped, i.e. the shorter wavelengths will arrive after the longer wavelengths. Correspondingly, after the propagation through the media with anomalous dispersion, the pulses will be negatively chirped, with the shorter wavelengths arriving before the longer wavelengths [114].

In the TA measurements, the onset of the signal will change if the chirp correction is not applied, so that the time zero will be wavelength dependent. An example of the effect of the chirp on the TA data can be seen in Figure 2.12. For the illustration of the chirp correction the data of the neat $Y 6$ material, measured for the side project [140], is provided. The sample was excited at $550 \mathrm{~nm}$ excitation, with the pump fluence of $10 \mu \mathrm{J} / \mathrm{cm}^{2}$. This dataset was chosen to demonstrate the effect of the chirp because of its low signal-to-noise ratio in the raw processing output and also the clear GSB and PIA signal areas that allow us to see the chirp.

Cross phase modulation. Cross phase modulation is a nonlinear process during which the intense pump pulse modulates the real part of the refractive index $n(t)$ of the transparent medium (equation 2.33). When pump and probe pulses overlap in space and time, the spectrally broadened pulse experiences this modulation and therefore the spectral distribution of the probe pulse is modified [102]. That may generate some unwanted signals around the time zero point.

In the ultrafast pump-probe experiment using the supercontinuum as a probe, the pump pulse is usually more intense than the probe. That means that any modification to the frequencies of the probe would be triggered by the pump pulse and 
not due to self-phase modulation. The intensity of the induced cross phase modulation signal depends on the chirp in a way that for the greater chirp the separation between the neighbouring frequencies in the probe will be more apparent and the cross phase modulation induced signal will be greater $[2,10,50,102]$.

Two photon absorption. Most optical media like liquids or optical solids are transparent when low-intensity radiation $\left(<10^{11} \mathrm{~W} / \mathrm{m}^{2}\right)$ is applied. However, when high power ultrashort laser pulses are used, the media can start absorbing through multi-photon absorption [102]. For sufficient excitation density in pump-probe experiments such as TA, the two photons can be absorbed simultaneously - one from the pump and one from the probe. This effect happens around the time zero point, where the pump and the probe temporally overlap and results in the appearance of a positive feature in $\Delta T / T$ signal.

It has been observed $[119,146]$ that two photon absorption happened in almost all organic solvents after the UV excitation (250-350 nm). The two photon absorption signal also appears earlier at the short wavelengths than at the longer wavelengths which comes from the chirp in the broadband pulse.

Stimulated Raman amplification. This effect might occur when the pump pulse is located spectrally close to one of the probe's wavelengths. That induces the change in the optical density at specific wavelengths. This effect, like many others reviewed here, only takes place at the temporal correlation of the pump and probe pulses [102].

\subsection{Transient grating photoluminescence spectroscopy}

Early studies of eumelanin [165] claim that the pigment does not fluoresce. However, it was proven incorrect later [121, 125, 126] which makes fluorescence spectroscopy an important tool in studying the pigment's complex behaviour.

The principal method, used before $[37,38]$ is the fluorescence upconversion spectroscopy $[66,181]$ which uses the nonlinear medium to generate an optical shutter. In this technique, there are two laser pulses, one of which, called the gate pulse, is delayed in time, and the other is used to excite the sample. After the excitation, the 
emitted fluorescence is overlapped with the gate pulse in a nonlinear crystal. The crystal is oriented in a way to satisfy the phase matching conditions for sum frequency generation for the gate and the collected fluorescence. The generated signal has a low background and high resolution. This method, however, is unable to detect broadband spectra, because the non-linear optical crystal is tuned to an angle that only activates upconversion for a specific wavelength. To obtain a photoluminescence (PL) spectrum it is required to scan the crystal angle to separately resolve different wavelengths [66], which affects the efficiency.

The alternative for that, the optical Kerr gate spectroscopy [9, 118, 155], has the capacity of recording the broadband PL spectra. In this technique, the generated fluorescence passes two crossed linear polarizers, that have an isotropic Kerr medium placed between them. The optical Kerr effect satisfies the phase matching conditions for all the PL wavelengths, which results in broadband PL spectra detection. Nevertheless, this method is limited by the transmission bandwidth and the extinction ratio of the polarizers that are supposed to suppress the signal background.

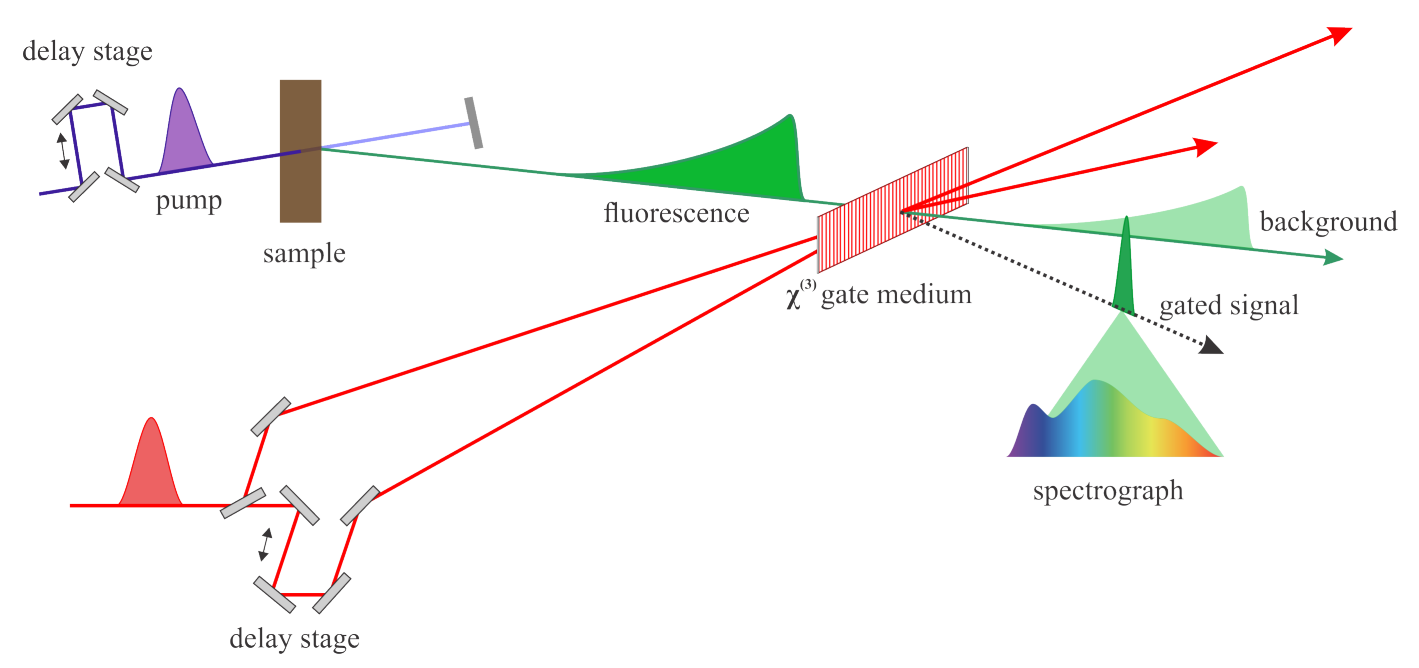

Figure 2.13: Schematic depiction of the broadband TGPL method.

The solution for the broadband ultrafast PL method is the transient grating photoluminescence (TGPL) spectroscopy method (Figure 2.13), developed by Chen et al. [31] of Victoria University of Wellington. This technique uses the approach of frequency-resolved optical gating for characterizing ultrashort laser pulses. The flu- 

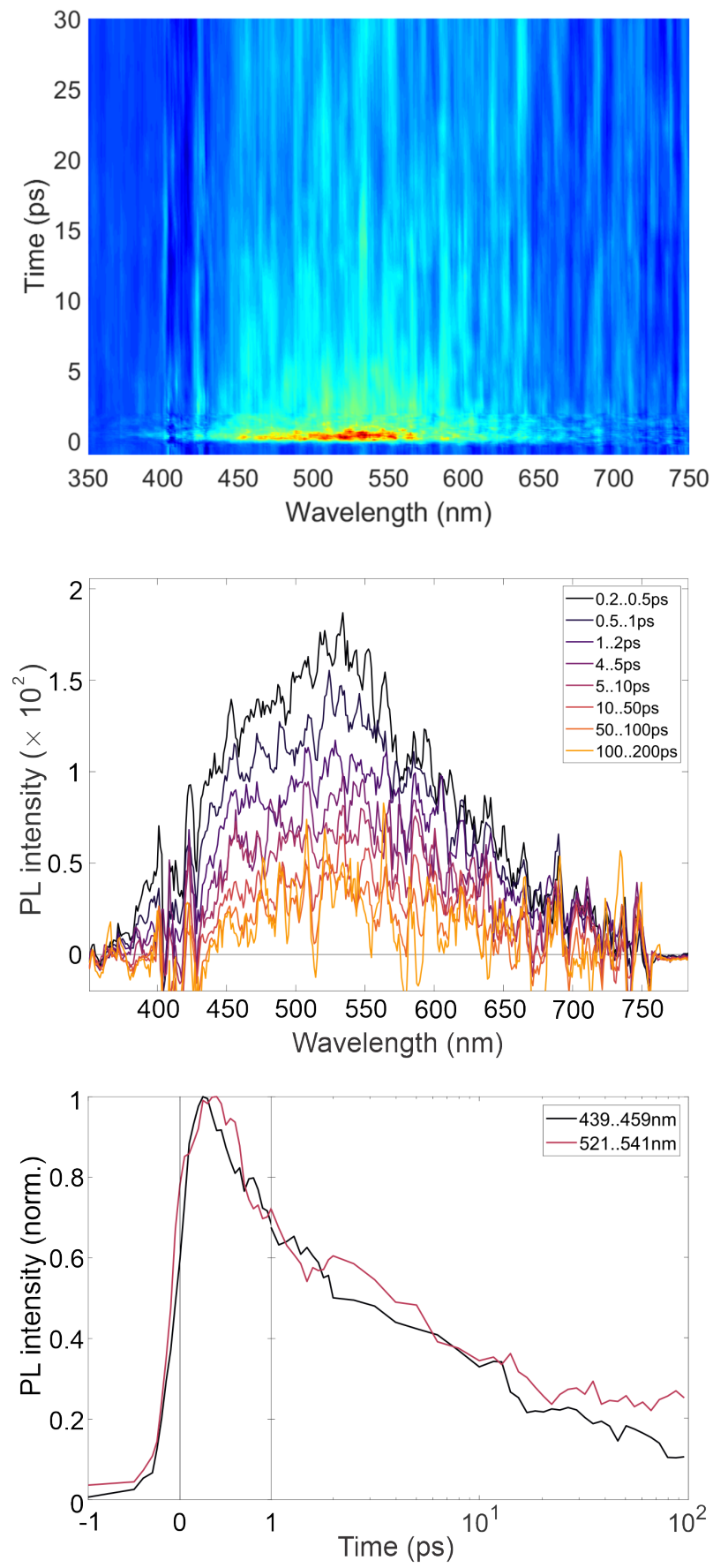

Figure 2.14: Example of TGPL data of eumelanin: surface (a), spectral traces (b) and kinetics traces (c). 
orescence is sampled using a transient diffraction grating, generated via interference of two gate pulses. This separates the gated PL signal from the ungated background. The optical Kerr effect modulates the refractive index of the gate medium. The ungated fluorescence is spatially blocked to eliminate the background.

In the TGPL experiment, there are two delay stages. One of them is ensuring the time resolution of the system, providing the temporal overlap of the sample fluorescence and the two gate pulses inside the Kerr medium. The second delay stage produces the temporal overlap of the two gate pulses at the grating medium. The diffraction grating, generated by two gate pulses, only exists in a moment of the temporal overlap of those two beams.

The TGPL signal is, like the TA signal, a three-dimensional matrix comprised of PL spectra and kinetics. To illustrate the result of one TGPL measurement the data for the synthetic eumelanin film after $267 \mathrm{~nm}$ excitation is chosen and presented in Figure 2.14. We take the slices in wavelength and time domains to obtain kinetics and spectral traces, respectively. All TGPL experiments, presented in this thesis were obtained in collaboration with Isabella Wagner of Victoria University of Wellington.

The efficient broadband detection of the existing system is 340-1300 nm [31]. By changing the crossing angle between two beams, the broadband detection can be shifted towards the UV range of the spectra, crucial for eumelanin studies. 
- Chapter 3

\section{Ultrafast spectroscopy of the main eumelanin building blocks DHICA and DHI}

\subsection{Chapter introduction}

Eumelanin in vivo occurs in a high degree of aggregation. Its high complexity and insolubility in most organic solvents obscure the information regarding the main deactivation mechanism. To address this question, it is important to use a bottom-up approach by studying the photochemistry of eumelanin building blocks. By understanding the processes and photochemical species behind the pigment's effective UV dissipation we might gain valuable information about the photophysical and photochemical behaviour of eumelanin. In this chapter, we will be revealing the decay dynamics of the key eumelanin precursors DHICA and DHI and their deactivation mechanism. This information will be later used to compare the revealed dynamics with eumelanin polymers.

\subsubsection{Photophysics of DHICA key building block}

The mirror-image rule violation observed in the DHICA spectra (Figure 3.1), is manifested in the lack of the shoulder around $296 \mathrm{~nm}$ in the emission spectrum. This shoulder, however, appears in one of the absorption peaks of DHICA. The observation of mirror-image rule violation in DHICA led to a hypothesis that this phenomenon is due to convergent adiabatic and non-adiabatic ESIPT processes [128]. The calculations led to a conclusion that upon photoexcitation of DHICA in aqueous solution at neutral $\mathrm{pH}$, the excited state can be deactivated through ESIPT or ESPT, involving phenol groups. From these considerations, it was first proposed that excited state proton transfer may be a primary ultrafast energy dissipation mechanism 
in the eumelanin pigment.

From Figure 3.1 we observe a large Stokes shift that shows internal conversion to the lowest excited state. This shift accounts for roughly $1 \mathrm{eV}$ of the excited state excess energy.

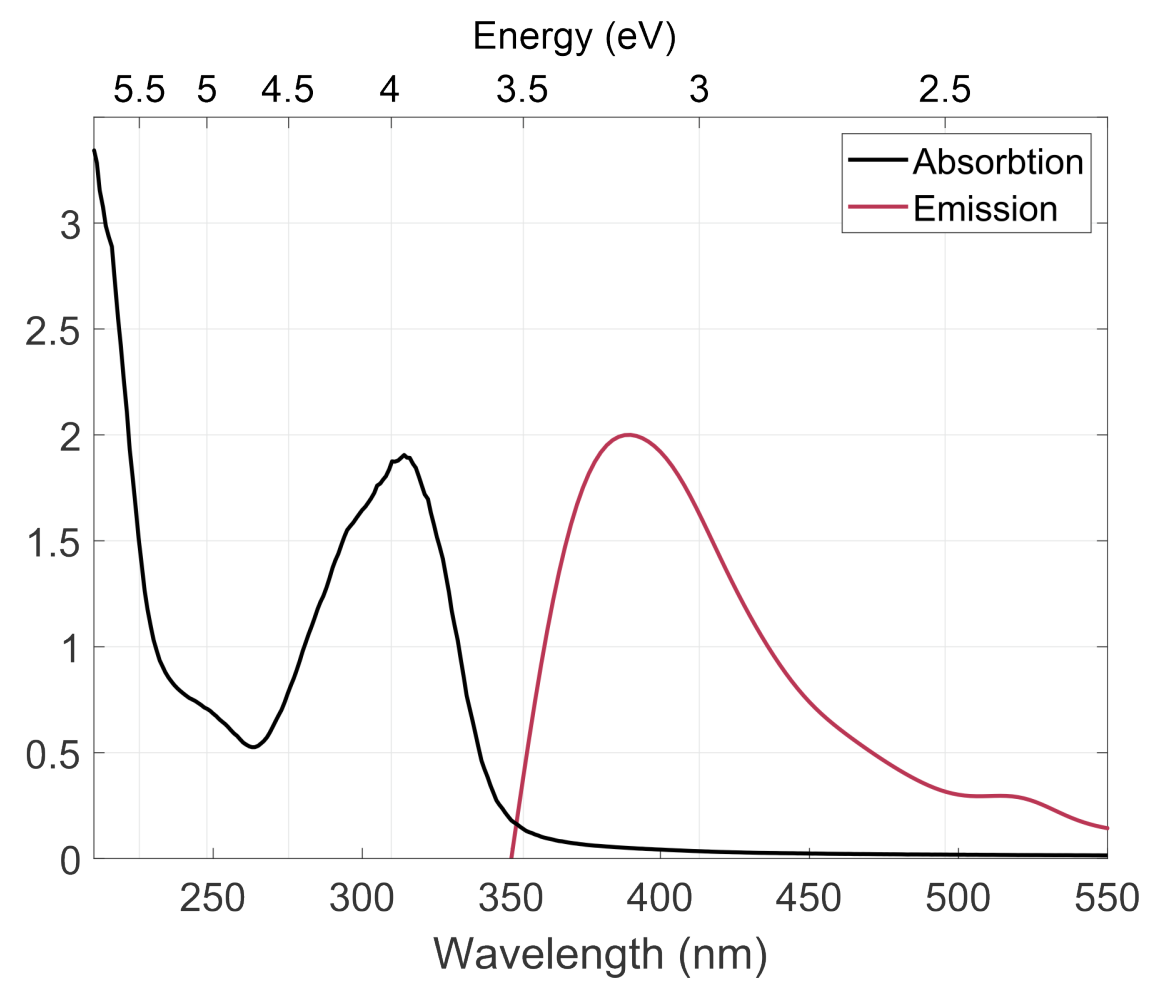

Figure 3.1: Mirror-image rule violation in DHICA monomer.

\section{Excited state intramolecular proton transfer}

The ESIPT process is facilitated if an intramolecular hydrogen bond exists in the ground state. In DHICA with the protonated carboxylic acid group, the $\mathrm{COOH}$ and $\mathrm{NH}$ groups can be involved in the ESIPT process by creating a hydrogen bond. It was already shown [11] for the indole-5-carboxylic acid and indole-2-carboxylic acid, related to DHICA, that there is an equilibrium between the neutral hydrogen bonded form and the zwitterionic form. Zwitterion (electroneutral molecule that has positively and negatively charged functional groups [161]) has the hydrogen from the carboxylic group transferred to the nitrogen. 
In acidic conditions, when the carboxylic acid group is protonated and produces the $\mathrm{HN}-\mathrm{HOOC}$ bond, the red-shifted zwitterion fluorescence is observed. Alkaline conditions, however, give rise to the anionic form, where no hydrogen bond can be formed, and show blue-shifted fluorescence. It was proposed [60] that DHICA also has the excited-state equilibrium between zwitterion and hydrogen-bonded form. The excited-state equilibrium is entailed to be fast due to the ultrafast character of the proton transfer (sub-picosecond to picosecond time scale [56, 94]). In acidic conditions, the short-lived red-shifted fluorescence was explained with the formation of the zwitterionic form. In neutral conditions, DHICA is present in its anionic form, resulting in the blue-shifted fluorescence, when the lifetime of the decay is three times larger than for the zwitterion.

It was noted by Corani et al. [38] that the surrounding solvent molecules play a crucial role in the excited state dynamics of DHICA monomers. The recorded fluorescence upconversion decay of the neutral form of DHICA at low $\mathrm{pH}$ was dominated by a major ultrafast $\sim 300 \mathrm{fs}$ component. Hence, in an aqueous solution, the ESIPT from the carboxylic acid group occurs within $300 \mathrm{fs}$ after excitation. When the fluorescence of DHICA was measured in methanol, the picture completely changed - the lifetime prolonged from 3.5 ns [38]. On the red side of the spectra, the fluorescence band experiences a fast rise, which was observed before when studying the excited-state solvation dynamics of methanol [151]. Therefore, this few picoseconds rise was attributed to a solvation process of the molecule in its excited state [38]. The observed slow excited-state decay demonstrated that ESIPT process is completely blocked in methanol. Such solvent sensitivity suggested that the surrounding solvent molecules are involved in the ESIPT process and their orientation might facilitate the proton transfer. Therefore, it was concluded that the water environment is essential for the proton transfer from $\mathrm{COOH}$ to $\mathrm{NH}$ group. The scheme, illustrating ESIPT process in the acidic aqueous environment, is shown in Figure 3.2.

\section{Excited state proton transfer from $\mathrm{OH}$ groups to the solvent}

In physiological conditions [72] that are the most relevant for our study, the carboxylic acid group is deprotonated. Therefore, the solvent-mediated ESIPT process 


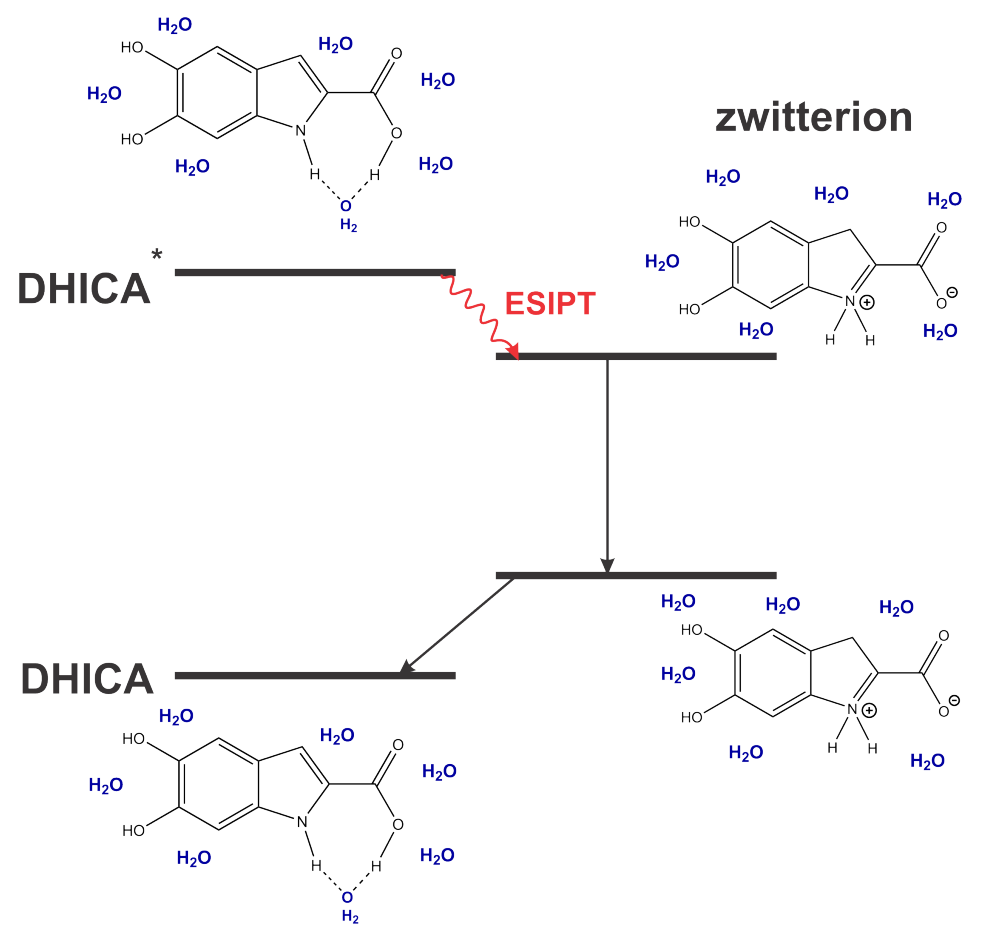

Figure 3.2: Excited-state intramolecular proton transfer in DHICA monomers in acidic environment.

is impossible since DHICA is in its carboxylate monoanion state.

For DHICA possessing two $\mathrm{OH}$ phenol groups, hydrogen bonding and further proton transfer are also possible. From earlier calculations by Olsen et al. [128] it was suggested that the phenol groups of DHICA are involved in ESIPT process or multistep ESPT to the solvent. The fluorescence lifetime of $\mathrm{DHICA}^{-}$varies depending on the molecule environment - in the presence of methanol it becomes almost three times longer than the one in the water environment. $\mathrm{DHICA}^{-}$also experienced 1 ps solvation dynamics process similar to that in methanol. These results suggest the slow ESPT process to the solvent [38].

According to the scheme in Figure 3.3, the dianion is formed as a result of the excited-state decay of monoanionic DHICA- through the ESPT process. When one or another phenol group was removed, the fluorescence decay became wavelengthindependent with a lifetime, similar to that of DHICA in methanol, in which proton transfer is forbidden [38]. Therefore, solvent dependence of the fluorescence spec- 


$$
\text { carboxylate monoanion phenolate dianion }
$$

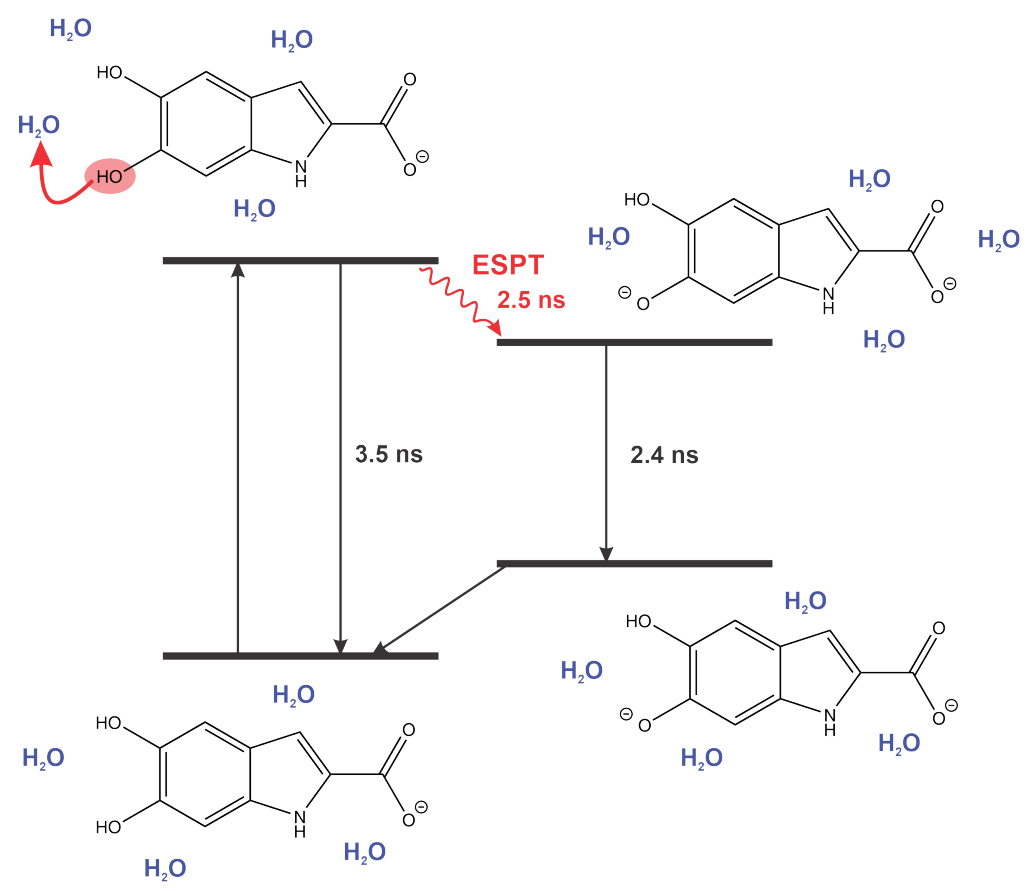

Figure 3.3: Proposed excited-state proton transfer to the solvent in DHICA monomers in neutral environment.

tra and kinetics mostly vanished. That indicated that the solvent dependence of carboxylate anion's fluorescence is related to 5- and 6-OH groups and not due to a general solvent dependence of the monomer's indole core [38] and serves as further proof of ESPT process in DHICA ${ }^{-}$.

For addressing the excited state deactivation of eumelanin and its precursors in the human body, we need to take into consideration physiological conditions. As was mentioned earlier in this section, under physiological conditions of the human body [72] carboxylic acid group of DHICA is deprotonated, leaving the molecule in its monoanionic state. If one claims that DHICA reflects the photophysics of eumelanin, its slow decay in neutral water cannot account for the pigment's photophysics. In that case, the proposed excited state proton transfer, observed in DHICA cannot be the decay mechanism for eumelanin.

We propose that the reason for such discrepancy could lie in possible wrong sam- 
ple preparation. It was shown that de-carboxylated form of DHICA, DHI tends to be highly oxidizable and unstable in the presence of oxygen [61]. The current experiments on DHICA, presented in literature $[38,60]$ were performed under aerated conditions. Considering that the DHI molecules are highly unstable in the presence of oxygen, there is a possibility that the DHICA might also polymerize in aerated conditions and therefore the data could be compromised. To avoid a possible oxidative degradation, we have focused on the more careful sample preparation with solvents degassing prior to the measurements.

\subsubsection{Photophysics of DHI key building block}

DHI is a highly oxidizable molecule, unstable to oxygen and alkali [61]. The studies of this key building block are complicated by the dark polymeric melanin-like impurities, present in DHI samples. This samples contamination complicates spectroscopic measurements of the molecule and slows down the progress of establishing the role of DHI in eumelanin energy dissipation.

$A b$ initio calculations by Sobolewski and Domcke [162] propose the excited state deactivation mechanism via non-adiabatic ESIPT from 5-OH group to the neighbouring carbon atom of the six-membered ring. This reaction is barrierless and therefore, very fast. The formed photochromic product 6-hydroxy-4-dihydro-indole5-one (HHI) and its forms absorb strongly across UV-Vis spectral range. The absorption spectrum of a mixture of DHI and HHI compounds exhibits monotonic absorption, increasing towards UV which is indicative of eumelanin so that it may play an important role in the energy dissipation mechanism.

Ultrafast TA measurements by Gauden et al. [61] could not come to a conclusion about whether HHI plays a primary role in DHI excited state deactivation due to the spectral range of their experiment $\left(440 \mathrm{~nm}-700 \mathrm{~nm}\right.$ whilst $S_{0}-S_{1}$ transition of HHI is centered at $391 \mathrm{~nm}$ ). Photoexcitation of DHI transfers the molecule to the singlet excited state, then the vibrational energy dissipation brings it to the equilibrated singlet excited state within 5-10 ps. This excited state experiences radiative decay with the time constant 140-180 ps to the ground state and nonradiative decay to the triplet state through the intersystem crossing. DHI radical cation is formed through 
photoionization through the equilibrated excited state, releasing the solvated electron [61]. ESIPT mechanism with the formation of zwitterionic form was proposed based on short excited state lifetime. However, direct evidence of that is lacking.

It was also shown [74] that UV dissipation mechanisms in DHI, like in eumelanin are dependent on the wavelength of excitation. Such behaviour was also observed in indole systems before. The origin of this phenomenon is explained as two competing processes: internal conversion to the lowest excited state and sub-ps electron transfer to the solvent [131, 164]; both of these processes might take place in DHI.

Later quantum chemistry calculations showed the possibility of the sequential proton-coupled electron transfer (PCET) in DHI molecules. The solvated electron is generated by the reorganization of the solvent after the vibrational relaxation of DHI to the lowest excited state, following UV excitation, and the subsequent electron transfer to the solvent. The formation of a solvated electron induces a proton transfer from the phenol group of DHI to a neighbouring solvent molecule. Both phenol groups are involved in this process, therefore multiple proton transfer processes might take place. DHI then relaxed to the ground state through internal conversion [127].

In this chapter we will explore the ultrafast radiative and nonradiative decay of DHICA and DHI monomers, maintaining the careful consideration of the probable polymerization that might have affected the results, presented in the literature. Understanding the real processes behind excited state deactivation of pure monomers will provide an insight into eumelanin's excited state behaviour.

\subsection{Ultrafast spectroscopy results of DHICA monomer}

DHICA monomers provide an important insight into eumelanin photophysics. After suggesting that proton transfer is the main excited state deactivation for DHICA monomers [38] literature reviews speculate the role of the proton transfer in the eumelanin energy dissipation. It was important to prevent a possible polymerization and provide robust data explaining DHICA behaviour. In this section, we will study the DHICA monomer behaviour in different experimental conditions using 
a combination of TA and TRPL techniques. The obtained results will be used as a benchmark for eumelanin energy dissipation studies.

\subsubsection{Sample preparation}

For the experiments, the powder of DHICA monomer (98\% purity) was purchased from $A K$ Scientific. $3 \mathrm{mg}$ of the powder was solubilized in $1.2 \mathrm{~mL}$ of MilliQ water to form a solution. For the methanol experiments, $2 \mathrm{mg}$ of DHICA powder were dissolved in $1.2 \mathrm{~mL}$ of methanol. The resulting solutions were sonicated for 10 minutes to break the large undissolved particles apart and then filtered through $0.2 \mu \mathrm{m}$ syringe filter. The concentration was set with absorbance at the excitation wavelength (267 $\mathrm{nm})$ of 0.3-0.6 in a $1 \mathrm{~mm}$ cuvette.

Water and methanol were degassed prior to the solution preparation using three consecutive freeze-pump-thaw cycles [58, 149]. The flask, containing the solution, was attached to the high vacuum tube. It was then placed in the cold trap and frozen with liquid nitrogen. With the water in its solid (frozen) state, the vacuum valve was opened, removing all the air above the sample. Then the liquid nitrogen was removed to let the sample thaw. After the sample thawed, the procedure was repeated until the gas bubbles stopped coming out of the solution. When all cycles were completed, the solution was sealed under the argon atmosphere.

\section{Sample preparation for $\mathrm{pH}-\mathrm{dependent}$ spectroscopy}

The protonation equilibrium can be described as

$$
H A \rightleftharpoons A^{-}+H^{+} ; K_{a}=\frac{\left[A^{-}\right]\left[H^{+}\right]}{[H A]},
$$

where $K_{a}$ is an equilibrium constant, known as an acid dissociation constant. HA is an acid that dissociates into the conjugate base $A^{-}$and hydrogen ion $\mathrm{H}^{+}$. From here, keeping in mind that $p H=-\log \left[H^{+}\right]$and $p K_{a}=-\log K_{a}$, the HendersonHasselbalch equation $[67,136]$ can be derived:

$$
p H=p K_{a}+\log \frac{\left[A^{-}\right]}{[H A]}
$$


The probability that the acid HA is protonated is given by $\langle x\rangle=[H A] /([H A]+$ $\left[A^{-}\right]$. From that

$$
\begin{aligned}
& {[H A]=\frac{\langle x\rangle\left[A^{-}\right]}{1-\langle x\rangle},} \\
& K_{a}=\frac{(1-\langle x\rangle)\left[H^{+}\right]}{\langle x\rangle}, \\
& p H-p K_{a}=-\log \frac{\langle x\rangle}{1-\langle x\rangle}
\end{aligned}
$$

From these, we can extract a protonation probability

$$
\langle x\rangle=\frac{10^{\left(p K_{a}-p H\right)}}{1-10^{\left(p K_{a}-p H\right)}}
$$

For the neutral environment, which is the most relevant condition, when studying processes happening inside the human body, the carboxylic acid group would be deprotonated. Using the equation (3.6) we can calculate that at $\mathrm{pH} 7$, the protonation probability of the carboxylic acid group $\left(p K_{a}=4.25\right)$ would be $0.2 \%$, while the protonation probabilities for the 5 and 6 phenol groups $\left(p K_{a}=13.2\right.$ for $5-\mathrm{OH}$ group and $p K_{a}=9.76$ for $6-\mathrm{OH}$ group) [29] will be $100 \%$ and $99 \%$, respectively. From this, it can be concluded, that in the neutral environment, when the ESIPT from carboxylic acid is impossible, the proton transfer from the $\mathrm{OH}$ groups might be the main dissipation mechanism.

To see the role of the different proton transfer mechanisms we have performed the $\mathrm{pH}$-dependent measurement of DHICA monomer. We studied three forms of DHICA, depicted in Figure 3.4, in three different environments. The neutral form of DHICA required the $\mathrm{pH}$ of the solvent to be 4 or less since the $p K_{a}$ of the carboxylic acid group is 4.25 . For that, we used the $\mathrm{pH} 3$ phosphate buffer, in which the protonation probability of the carboxylic acid group is $95 \%$, and it was assumed that in this form ESIPT would be a dominating excited-state decay pathway. For the study of the mostly deprotonated DHICA dianion, which presumably represents the result of the ESPT process in neutral water, we used basic water. It was prepared as an aqueous solution of sodium hydroxide $(\mathrm{NaOH})$ as a solvent. The $\mathrm{NaOH}$ aqueous 
acidic conditions<smiles>O=C(O)c1cc2cc(O)c(O)cc2[nH]1</smiles>

DHICA neutral neutral conditions<smiles>O=C([O-])c1cc2cc(O)c(O)cc2[nH]1</smiles>

DHICA carboxylate monoanion

alkaline conditions<smiles>O=C([O-])c1cc2cc(O)c([O-])cc2[nH]1</smiles>

DHICA phenolate dianion

Figure 3.4: Studied forms of DHICA monomer in the solvents of different $p H$.

solution ( $\mathrm{pH}$ 12) was used to deprotonate 6-OH group - in these conditions $99.4 \%$ of the molecules have their 6-OH group deprotonated, creating dianion. The solvents were degassed using the freeze-pump-thaw technique, and then the monomer powder was dissolved in them. DHICA has good solubility in high pH solutions, therefore there was no need to sonicate or filter the resulting solution. However, in $\mathrm{pH} 3$ buffer the solubility was lower and therefore the solution was sonicated and then filtered to avoid scattering on the undissolved particles. Figure 3.5 shows the absorption and steady-state emission spectra of the samples in different solvents.

Upon deprotonation of the carboxylic acid group, we observe a slight blue shift in the maximum of the spectrum. This shift has also been observed for indole2-carboxylic acid [75] and described as a result of decreased oscillator strength of the lowest $S_{0}-S_{1}$ transition upon deprotonation of the carboxylic acid group [37]. When DHICA is in a dianionic state (carboxylic acid and 6-OH groups are deprotonated), a strong red shift is detected. The shape of the absorption spectrum for the dianionic DHICA differs the most from all of the others. Mainly, the "shoulder", 


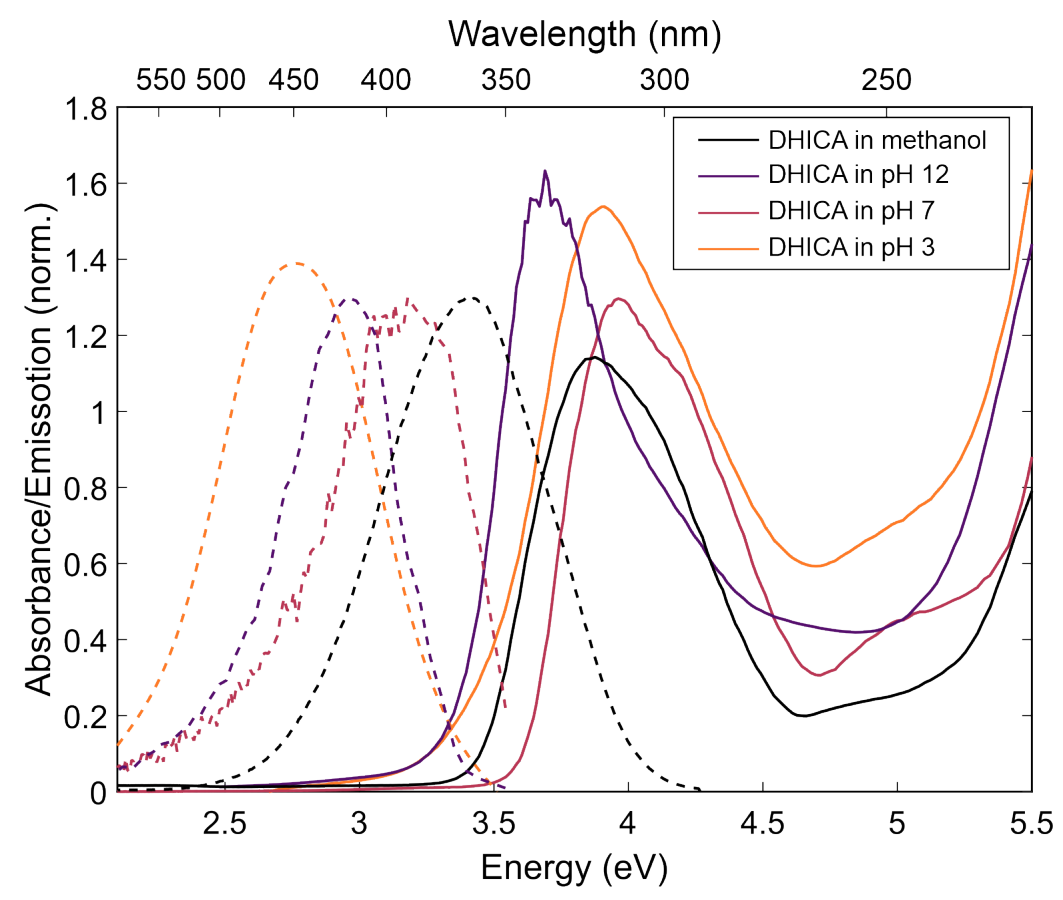

Figure 3.5: Steady-state absorption (solid lines) and emission (dashed lines) spectra of the DHICA monomer in different solvents.

mentioned earlier, which is faintly pronounced in the absorption spectra of DHICA in water and methanol, is lacking completely in the high $\mathrm{pH}$ spectrum.

\subsubsection{DHICA as photoacid}

We observe that DHICA loses a lot of energy (roughly $1 \mathrm{eV}$ ) on internal conversion until it reaches the lowest excited state. That accounts for a lot of excess energy to drive the proton transfer reaction. Hence we can calculate the driving force for the proton transfer using Förster analysis.

It is predicted that any proton containing molecule can be a photoacid if its $p K_{a}$ in the ground state is larger than $p K_{a}^{*}$ in the excited state. This indicates that in the excited state the molecule will be a stronger acid than in the ground state and tend to lose a proton in the excited state [171].

The spectral shifts that take place upon dissociation of the photoacid can be used to calculate $p K_{a}^{*}$ in the excited state. If we assume that the entropy change for excited 


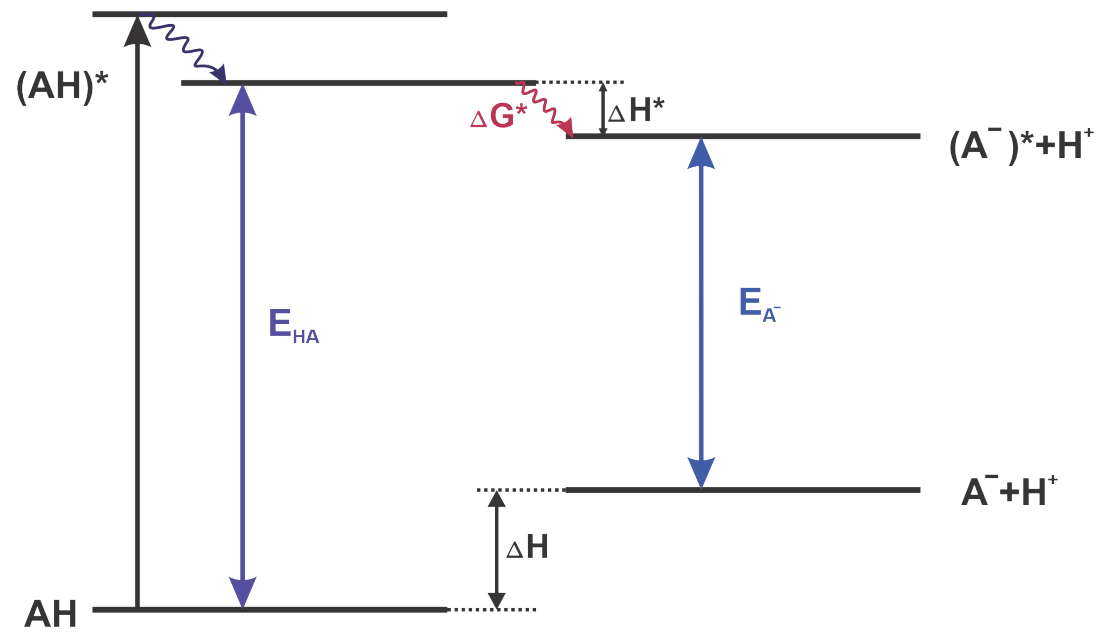

Figure 3.6: Proton transfer process in photoacids (the Förster cycle) in neutral conditions.

and ground state is the same, then the thermodynamic cycle (Figure 3.6) to describe the change of $p K_{a}^{*}$ will be described by Förster equation $[57,96,171,175]$

$$
p K_{a}^{*}=p K_{a}-\frac{E_{H A}-E_{A^{-}}}{2.303 R T}
$$

where $R$ is gas constant and $T$ is temperature. Hence, using the $p K_{a}$ for 6-OH group of DHICA carboxylate monomer equal 9.76, we calculate the excited state $p K_{a}^{*}=$ 4.69. Such a difference between the two dissociation constants indicates that the molecule is prone to the proton transfer in its excited state.

From these calculations, we can also conclude that in acidic conditions when the carboxylic acid group is protonated, the 6-OH group is not as strong photoacid as it is in neutral conditions. That demonstrates that in acidic conditions DHICA tends to give away the proton from the carboxylic acid group, but not from 6-OH group.

From there we can calculate the free energy of the proton transfer in the excited state [135]

$$
\Delta G^{*}=2.303 R T p K_{a}^{*}
$$

The resulting value of $\Delta G^{*}=0.3 \mathrm{eV}$ is the calculated proton transfer driving force. 


\subsubsection{The effect of the solvent environment on DHICA excited- state decay}

DHICA monomers experience two different excited-state decay pathways - through the excited state intramolecular proton transfer from carboxylic acid group to $\mathrm{NH}$ group; and excited state proton transfer from $\mathrm{OH}$ groups to the solvent. To resolve each of these pathways and explore the role of the proton transfer from the $\mathrm{OH}$ groups more closely, we studied DHICA in different solvents. DHICA in water is a monoanion, with the deprotonated carboxylic acid group. This form can only experience the excited state proton transfer from $\mathrm{OH}$ groups to the solvent.

After giving the proton to the solvent, DHICA is thought to transit to its phenolate dianion form. From there, the molecule relaxes to the ground state of the dianion and then transfers into the initial carboxylate ground state via proton scavenging from the solvent. When we study DHICA in basic water solution, the molecule would initially be a dianion. Therefore, considering that the DHICA undergoes the 4-level excited state deactivation through dianion formation, the excited-state decay of monoanion and dianion forms should be similar, unless reverse proton transfer from the same proton that just left from $\mathrm{OH}$ group takes place. By comparing the decays of the two forms with protonated and deprotonated $\mathrm{OH}$ groups we can establish the role of the ESPT in DHICA excited state deactivation (Figure 3.7, bottom right).

DHICA, dissolved in methanol, gives an insight about the excited-state decay of DHICA monomer, in which ESPT is suppressed, because methanol is a poor proton acceptor compared to water $[159,171]$. The excited-state decay of the DHICA in methanol (Figure 3.7, bottom left), compared with that of the DHICA in water will give another idea about the role of the proton transfer mechanism.

In acidic conditions, DHICA monomers undergo ultrafast excited-state decay through ESIPT process. Therefore, the comparison of the decays of the fully protonated DHICA and carboxylate monoanion will reveal the difference between the decay rates of the ESIPT from the carboxylic acid group and ESPT from OH groups, which we believe is the most important deactivation mechanism, as it is active in 
the neutral conditions of the human body.

Transient absorption results of DHICA monoanion in degassed water, presented in Figure 3.8, revealed that the excited state of a monomer decays in the ultrafast picosecond timescale. We observe a single PIA peak at $\sim 490 \mathrm{~nm}$ that almost completely decays during the measurement scan time (1 ns). The excited-state decay kinetics is demonstrating a rather fast decay - we see that the majority of the signal decays within the first few picoseconds. In water, the excited state proton transfer is only possible from the phenol groups to the surrounding solvent. The excited state lifetime $\tau$ was calculated using the weighted average value of the two-exponential fit:

$$
\tau=\frac{A_{1} * \tau_{1}+A_{2} * \tau_{2}}{A_{1}+A_{2}}
$$

where $A_{1}, A_{2}$ are the amplitudes of each exponent and $\tau_{1}, \tau_{2}$ are the exponential decays' lifetimes. Our results demonstrate the weighted average lifetime of the monomer at $4.25 \mathrm{ps}$. That suggests that the excited state deactivation of monomer, happening via excited state proton transfer from $\mathrm{OH}$ groups to the solvent, is faster than reported previously.

The presented ultrafast excited state deactivation reveals the importance of the ESPT from $\mathrm{OH}$ groups to the solvent. Ultrafast photoluminescence measurements of DHICA monoanion also revealed an ultrafast photoexcitation decay in the first few picoseconds. Interestingly, we observe an excited-state decay, relatively comparable with TA decay (Figure 3.9), which suggests that the TA probes the electronic excited states.

The role of the excited-state intramolecular proton transfer in DHICA can be studied when DHICA is dissolved in the degassed acidic buffer to ensure that the carboxylic acid group is protonated so that the proton transfer to the NH group is possible. This pathway was proposed to be the main dissipation mechanism in DHICA building block and eumelanin pigment since the excited-state decay rate of the neutral DHICA was substantially higher than that of the DHICA monoanion.

The study of the neutral form of DHICA monomer in degassed $\mathrm{pH} 3$ phosphate buffer revealed the same ultrafast excited-state decay as we observed for DHICA 


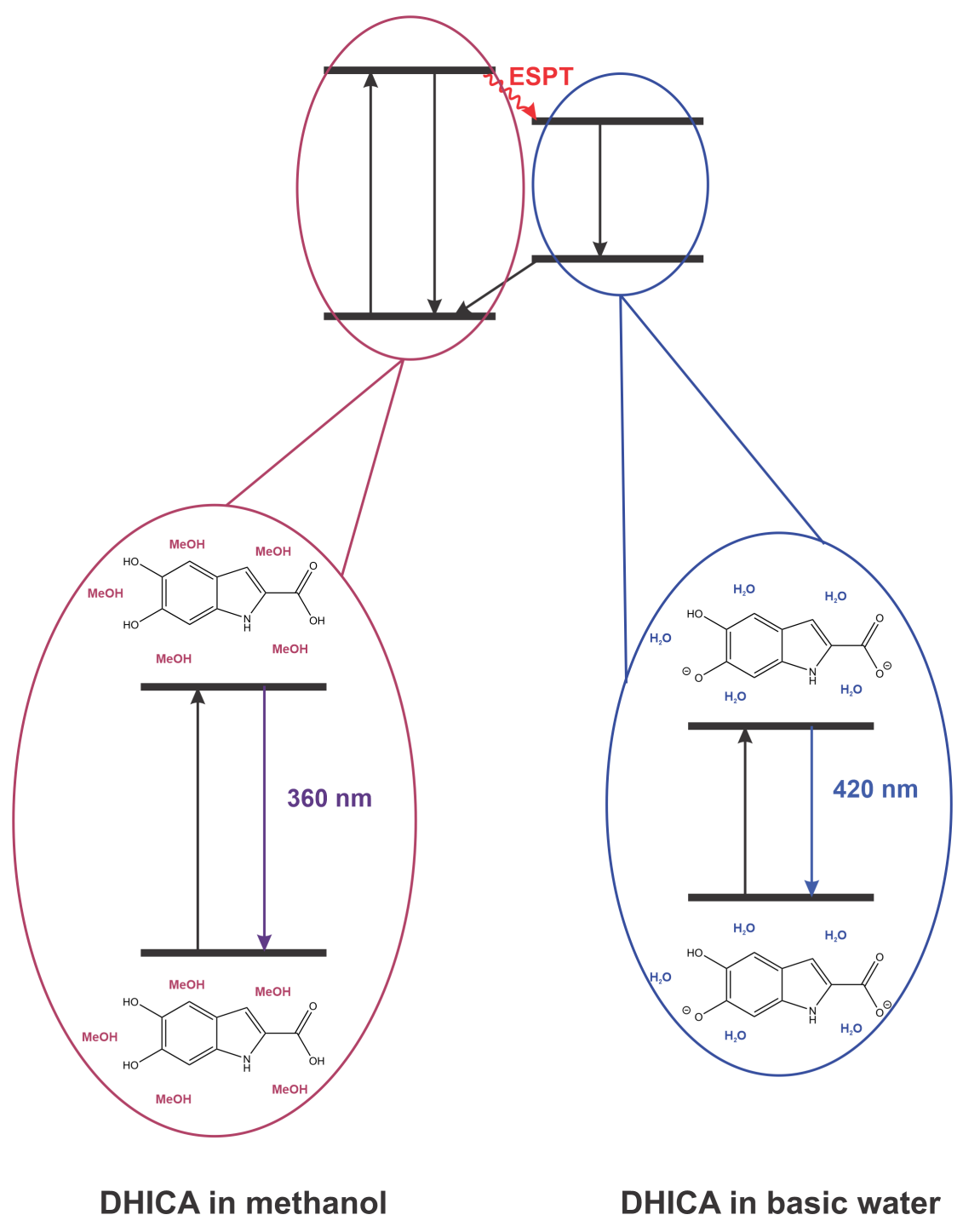

Figure 3.7: Break-down of the 4-level system into parts to study each of them. 

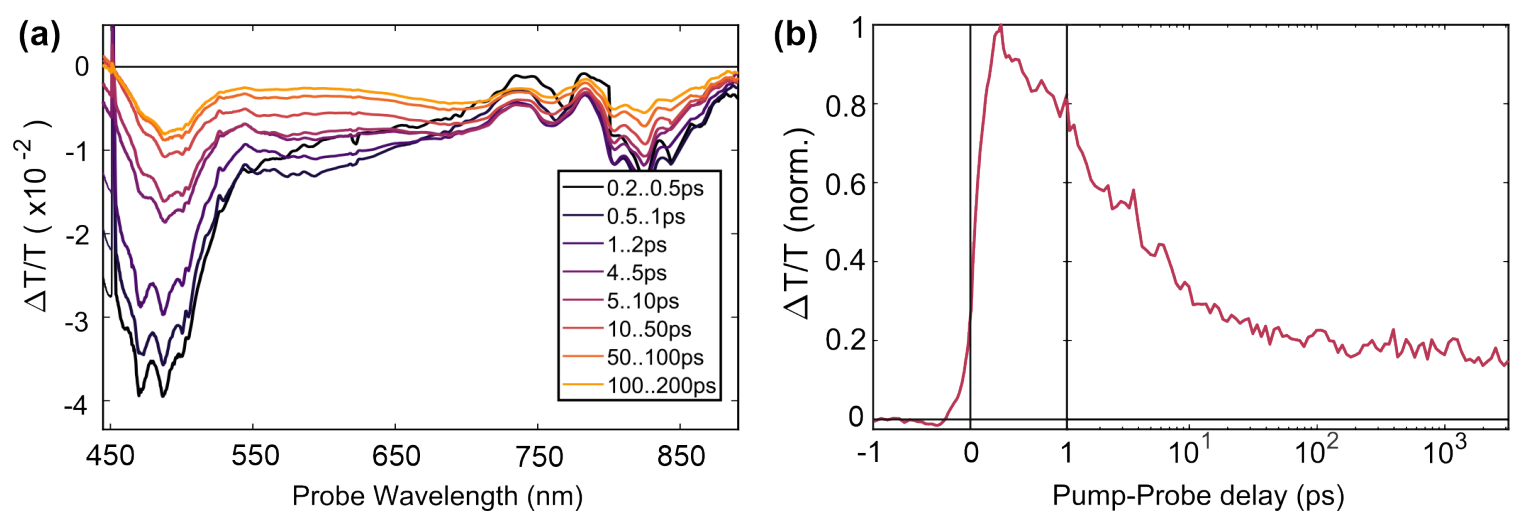

Figure 3.8: Transient absorption spectra (a) and kinetics (b) of DHICA monomer in degassed water.

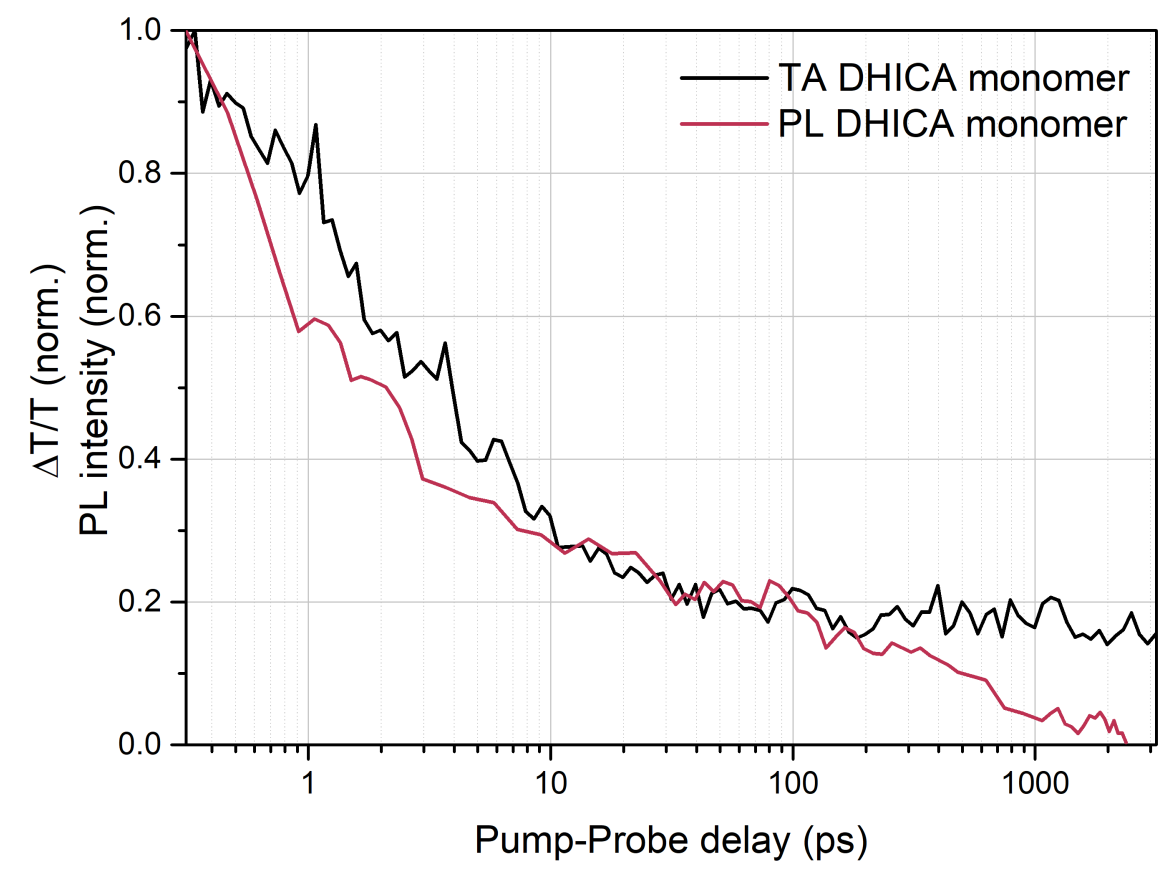

Figure 3.9: Comparison of the TA and PL decay of DHICA monomer in degassed water. 


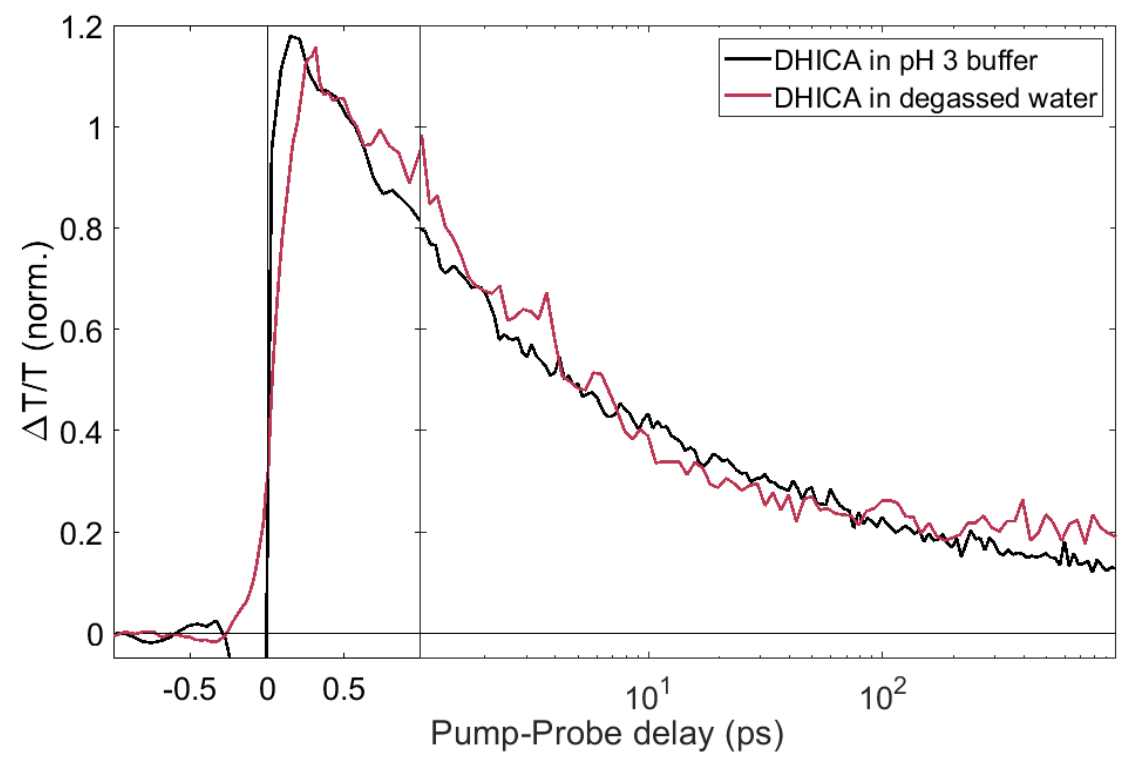

Figure 3.10: Comparison of of the transient absorption signal decays for the neutral DHICA and carboxylate monoanion.

in neutral degassed water. As shown in Figure 3.10, the two forms are decaying at the same rate. This indicates that the ESIPT from the carboxylic acid group to NH group is not, in fact, more important than the ESPT from $\mathrm{OH}$ groups to the solvent. The similarity of the two decays means that the deprotonation of the carboxylic acid group does not affect the excited state deactivation rate. This observation proves the importance of the ESPT from the $\mathrm{OH}$ groups to the solvent in the excited state deactivation of DHICA monomers and possibly of the eumelanin pigment.

To understand the proton transfer process role in the excited state deactivation of DHICA we studied two other possible forms of DHICA monomer - the phenolate dianion (Figure 3.7, on the right), and DHICA in methanol where proton transfer is suppressed (Figure 3.7, on the left). Dianion is supposedly representing the intermediate form of the 4-level ESPT process (Figure 3.3) in neutral water, therefore it is important to study this form on its own. DHICA in methanol, on the other side, will provide an insight into the excited-state decay rate of DHICA in the environment where proton transfer is suppressed.

From the kinetics traces comparison in Figure 3.11 (b) we observe that the excited 

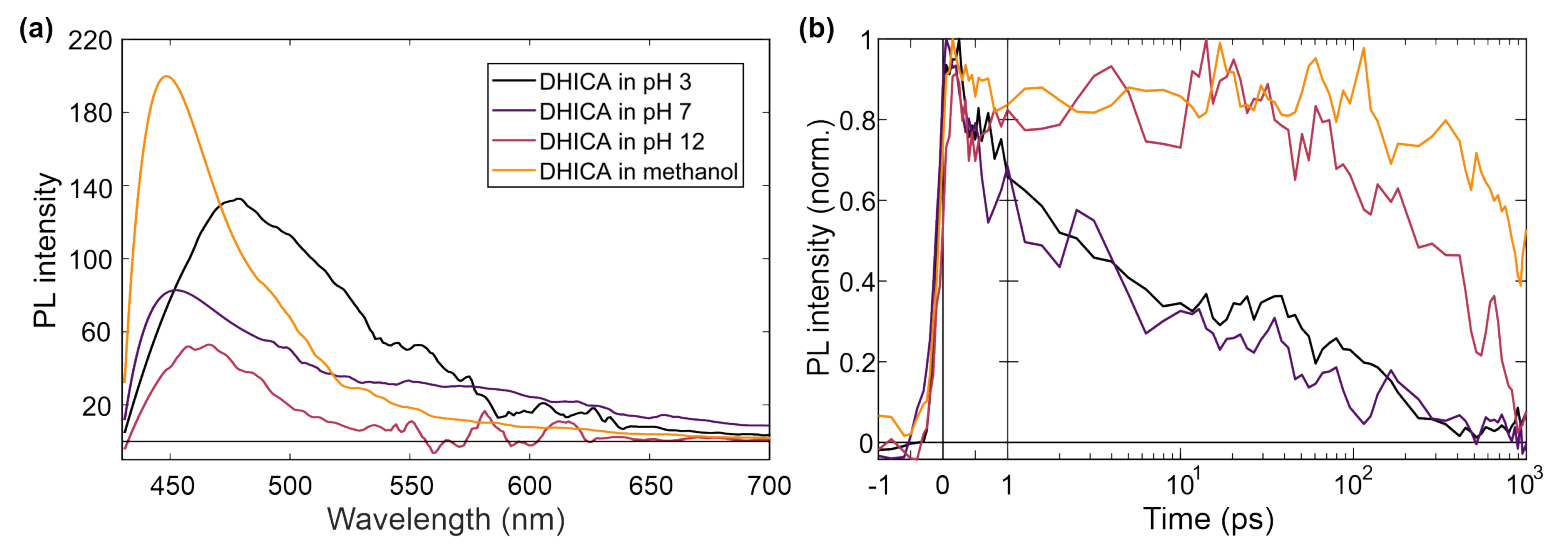

Figure 3.11: Comparison of TRPL results for different forms of DHICA.

state of DHICA in methanol does not decay within the time frame of the experiment (1 ns). This confirms the previously proposed hypothesis that DHICA does not undergo excited state proton transfer when dissolved in methanol, which leads to a drastic increase of the excited state lifetime from a few picoseconds to nearly $1 \mathrm{~ns}$.

The comparison of the decays of DHICA monoanion and dianion showed that the deprotonated DHICA form exhibits a rather slow decay when compared to the kinetics of DHICA with protonated $\mathrm{OH}$ groups. This shows that the deprotonated form itself undergoes slow relaxation, and therefore the 4-level model proposed in Figure 3.3 is not exactly correct. From what was observed in the measurements, the DHICA monoanion gets to the ground state within a few picoseconds and does not remain on the excited state within nanoseconds which would have happened if the 4-level system was correct. In the 4-level model DHICA monomer would undergo the decay through the decay of the deprotonated form, however, since it was shown to be slow, while the actual monoanion decay is fast, the 4-level model should be revised. We assume that in neutral conditions DHICA exhibits proton transfer from $\mathrm{OH}$ group to the solvent, and this transition has a large amplitude motion to access potential energy surface crossing to the ground state within a few picoseconds. The proposed mechanism is illustrated in Figure 3.12.

The possible explanation of the fast excited-state decay of the protonated DHICA monoanion compared to that of deprotonated dianion might be the rapid recovery of the proton that left the $\mathrm{OH}$ group. We assume that the proton that was dissociated 

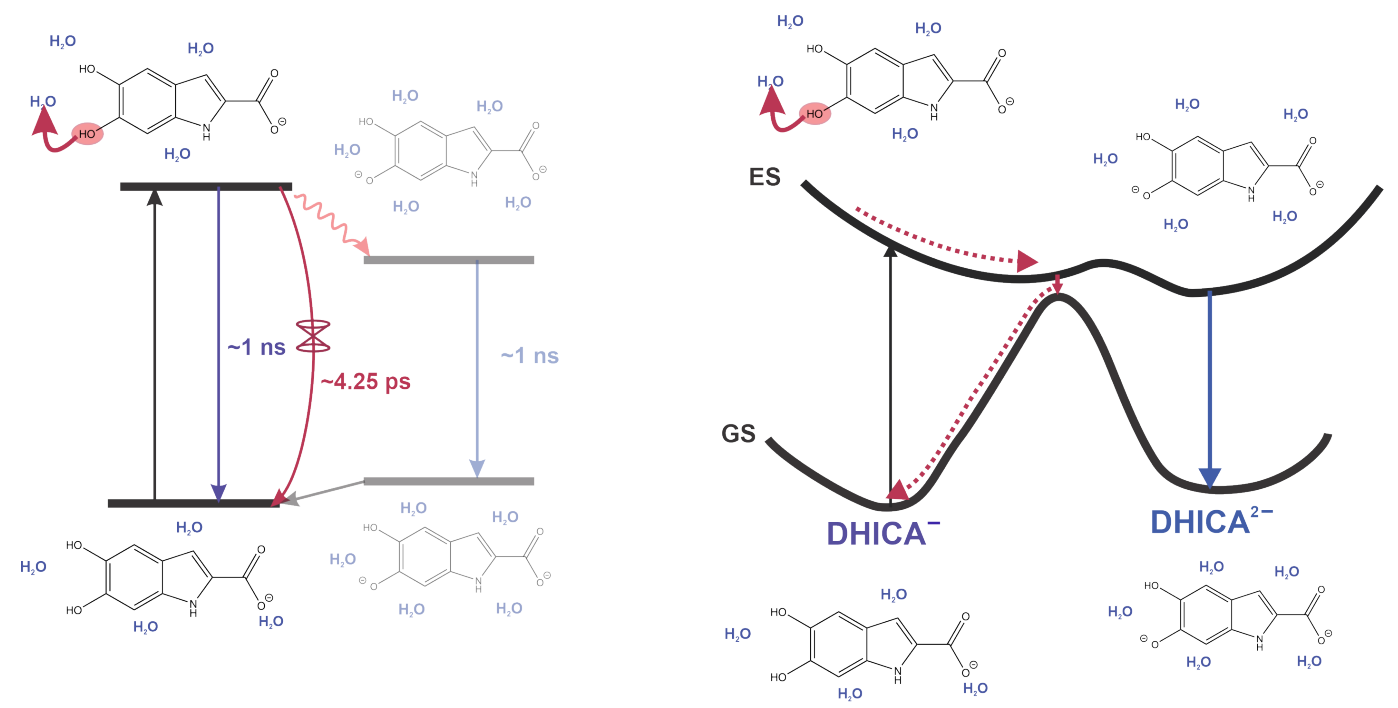

Figure 3.12: Schematic depiction of the proposed excited state deactivation via ESPT (on the left). The right panel proposes the depiction of the ESPT process in DHICA monomer for potential energy surfaces.

from the $\mathrm{OH}$ group remains in close proximity to the molecule and is then rapidly returned to the $\mathrm{OH}$ group from the solvent. The negative charge that was created due to the deprotonation of the $\mathrm{OH}$ group and the proton in the solvent form a geminate pair. The negative charge keeps the proton close and then can attract it back. In basic conditions there is no proton to give away, therefore the decay rate is substantially lower.

Taken those observations together we can conclude, with a high degree of certainty, that the DHICA monomers in physiological conditions, while measured with high accuracy to prevent polymerization, exhibits an ultrafast excited state proton transfer from $\mathrm{OH}$ groups to the surrounding solvent by experiencing rapid potential energy surface crossing.

\subsubsection{Radical formation in alkaline conditions eliminates the likely role of PCET}

Photoluminescence spectra of DHICA in water and $\mathrm{NaOH}$ depicted in Figure 3.13 (a) show the unexpected double-peaked photoluminescence signal. The first peak, 

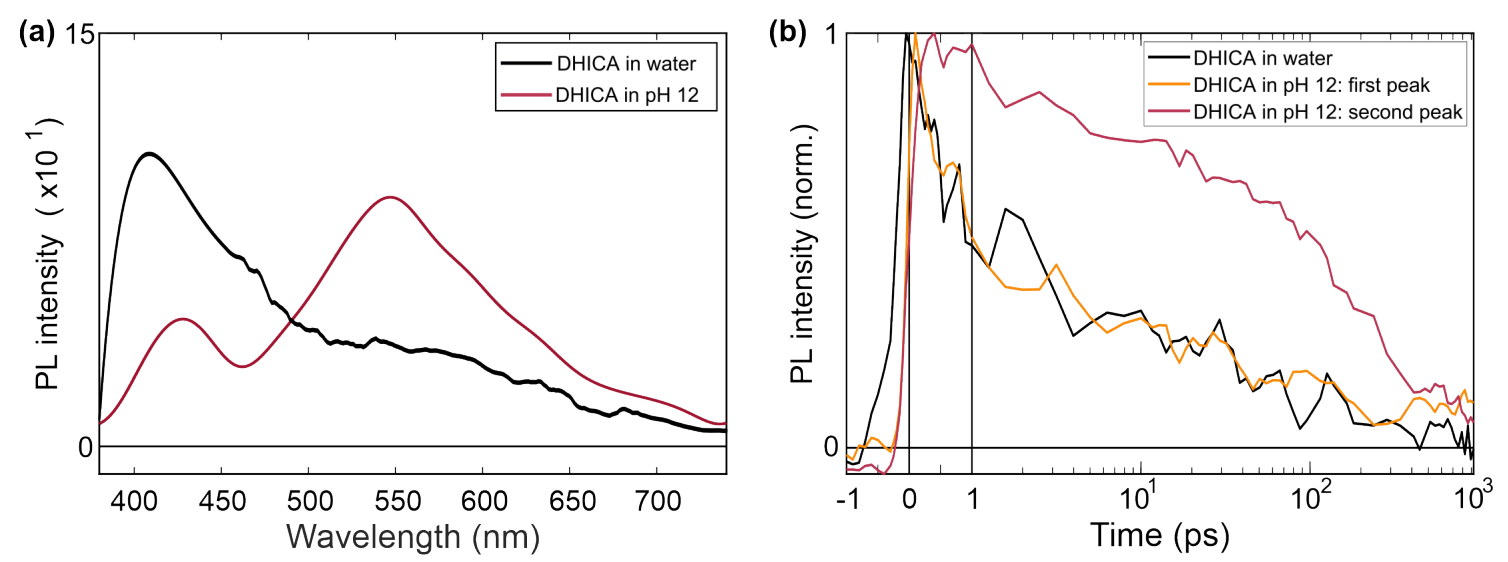

Figure 3.13: Comparison of TRPL spectra (a) and kinetics (b) of DHICA in neutral and alkaline conditions.

matching the one we observed with steady-state PL measurements and ultrafast PL results at low fluence, has a relatively fast excited-state decay - comparable with DHICA in neutral water. However, the second peak, appearing at the long wavelengths, shows a rather slow excited-state decay in order of $1 \mathrm{~ns}$.

The steady-state emission peak at $\sim 420 \mathrm{~nm}$ was recorded at the relatively weak excitation intensity. To figure out which peak in time-resolved PL measurement is the most relevant, we performed a series of intensity-dependent steady-state emission measurements on the TRPL setup. The results are presented in Figure 3.14. We see that at the low excitation power the "main" peak at $420 \mathrm{~nm}$ is dominating, but as we excite the sample at the higher pump powers, the second peak at $550 \mathrm{~nm}$ becomes more and more pronounced. To ensure that the sample did not degrade and the long wavelength peak is not a product of the DHICA polymerization, after measuring the sample at the relatively high power, we recorded the lowest excitation power spectrum again. The low power spectrum at the beginning and the end of the measurements showed no apparent difference and no additional peak that could have arisen as a result of oxidative polymerization. Therefore, we could agree that the additional $550 \mathrm{~nm}$ peak was a photoproduct of DHICA dianion and not a result of the possible oxidative polymerization of the sample.

The apparent fluence dependence of the steady-state emission spectra raised a question about the robustness of the already discovered excited-state decay of 


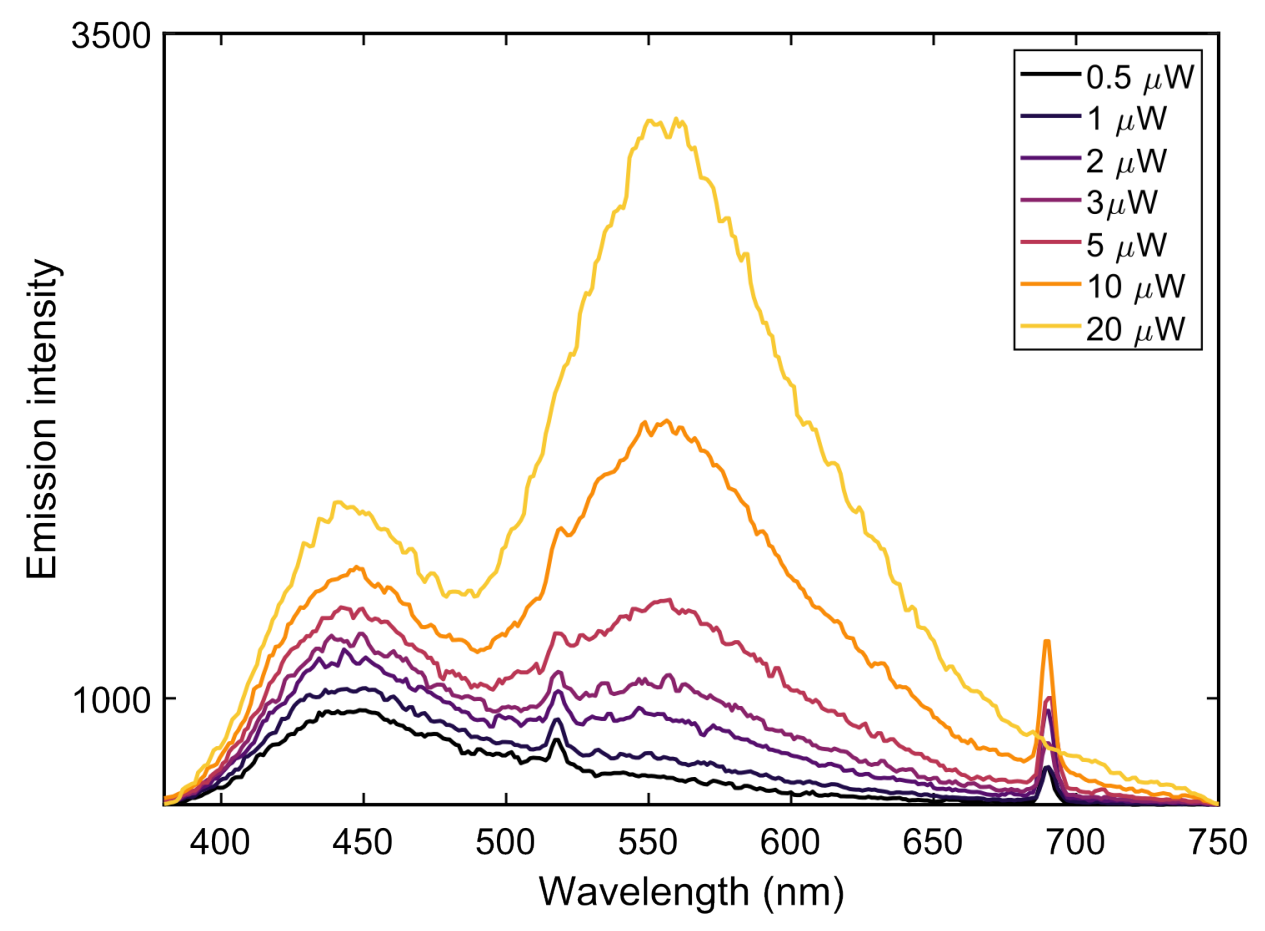

Figure 3.14: Time integrated intensity-dependent steady-state emission.

the main photoluminescence peak of DHICA phenolate. We performed a series of fluence-dependent TRPL measurements on DHICA in basic water solution. We observe the change of ratio between two peaks with the change of the excitation fluence - as the excitation power increases, the second peak becomes more pronounced with respect to the first main peak. This is illustrated in Figure 3.15 (a); the spectra are normalized to the first peak's intensity for clarity. We see that under the lowest fluence the second peak is negligible and cannot be separated from the noise, resulting from the laser's second harmonics influence. When excited at the high fluence, the first peak almost disappears. Additionally, we observe fluence dependence of the kinetics of the first peak - it decays substantially faster when excited with a more powerful pump. Therefore, the real kinetics, observed in section 3.2.3, obtained by exciting the sample at the lowest fluence $0.88 \mu \mathrm{J} / \mathrm{cm}^{2}$ was the real kinetics of the excited state of the DHICA dianion.

We assume that under alkaline conditions DHICA phenolate is able to transfer an electron from the 6-membered ring to the solvent and to turn into a radical. In 

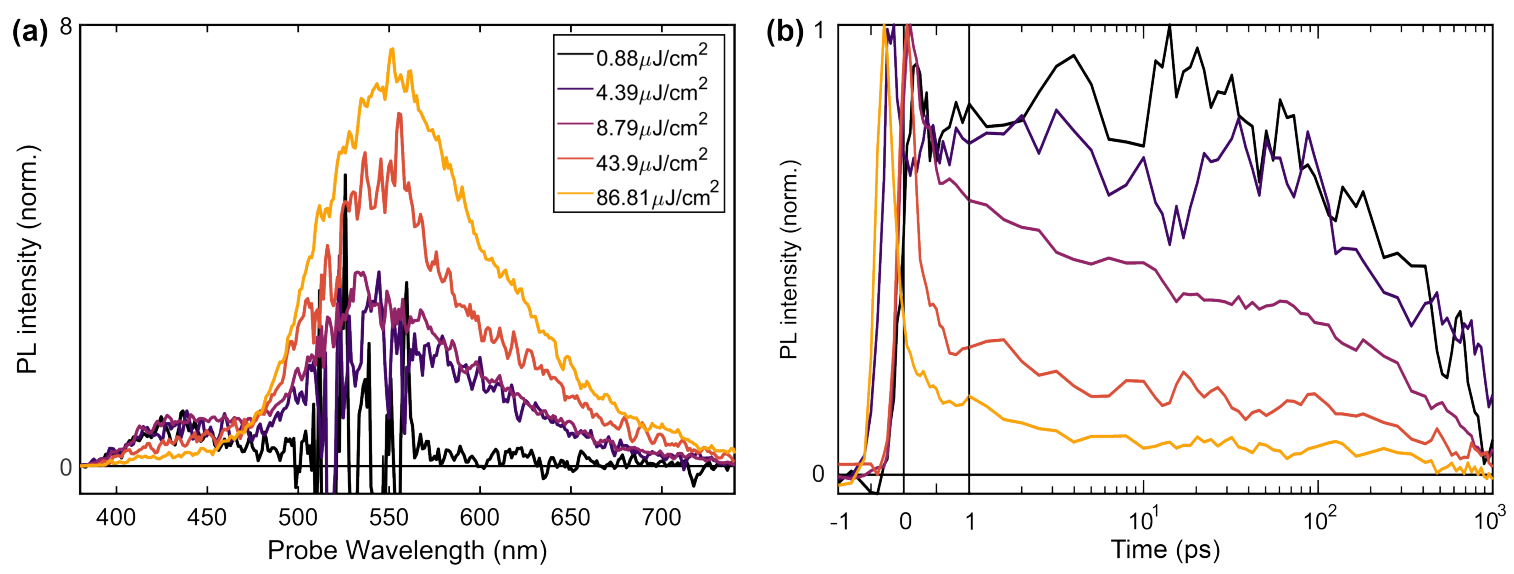

Figure 3.15: Fluence-dependent TRPL spectra, normalised to the first peak's intensity (a) and kinetics of the first peak (b).

the dianion state, the molecule is prone to electron transfer, so after an electron is released to the solvent and solvated electron is created, it can absorb an additional photon within the same laser pulse ( $\sim 100 \mathrm{fs}$ ) and get re-excited. The intensity of the formed peak exhibits a nonlinear dependence on the incident power (Figure 3.16). This double excited state is hypothesised to be emissive at $\sim 550 \mathrm{~nm}$. Therefore, by exciting the sample with the more powerful pump, we allow the molecule to absorb more photons, hence the formed radical will be emitting stronger. After losing an electron the emissive state geometry reorganises resulting in the red-shifted emission. The proposed mechanism is shown in Figure 3.17.

When looking at TRPL surface for DHICA in basic water (Figure 3.18 (a)), excited at the highest fluence for the clearer picture, when the radical form is dominating, we mostly see the strong peak at $\sim 550 \mathrm{~nm}$, because the intensity and lifetime of the first main peak is negligible when compared to the radical emission. However, when we zoom in the first 20 ps after excitation (Figure 3.18 (b)), we see the fast red shift of the initial peak. Therefore, when we excite DHICA in alkaline solution, the molecule's electronic structure reorganises and we observe fast bathochromic shift to the radical emissive form like shown in Figure 3.17, and hence the main peak's decay appears to become faster with a more powerful pump.

Time-dependent density functional theory calculations, performed by Dr Paul 


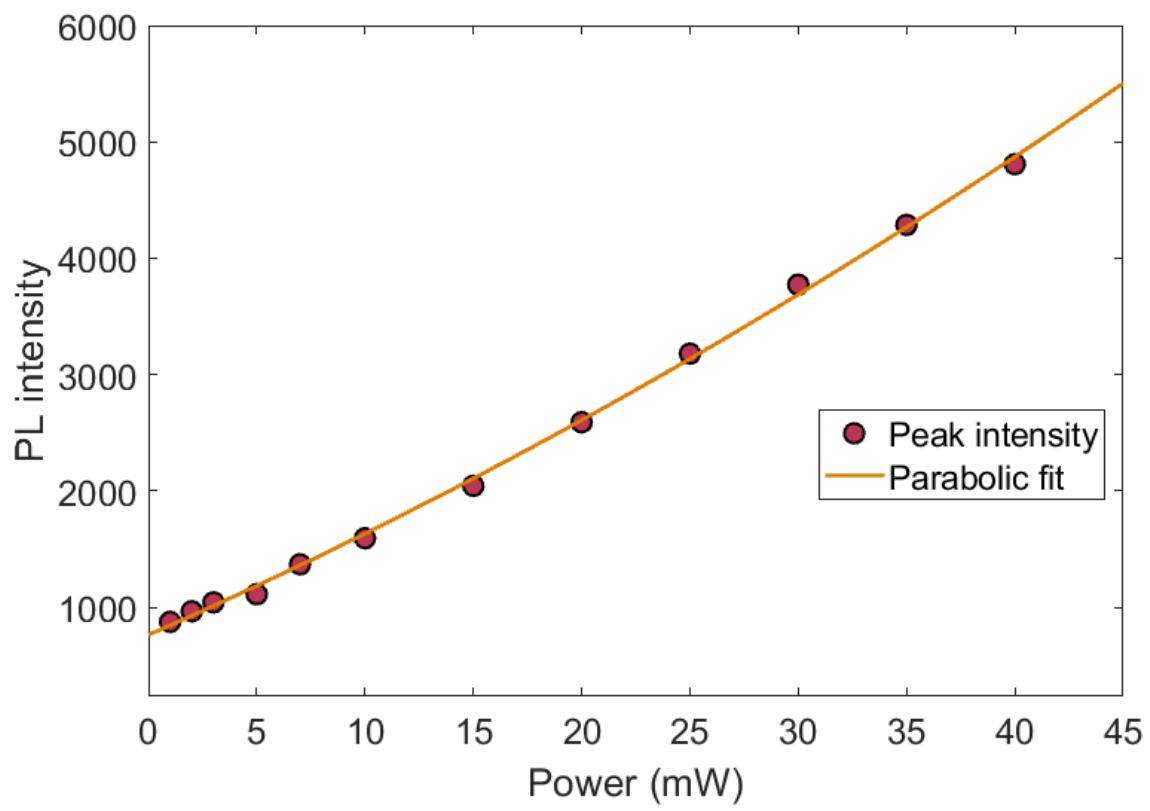

Figure 3.16: Nonlinear power dependence of the second PL peak.

Hume of Victoria University of Wellington confirmed the formation of radical species with the strongly red-shifted emission. The calculations demonstrated that when dianion loses an electron which transforms it into a radical form, it experiences a strong bathochromic shift from $316 \mathrm{~nm}$ to $474 \mathrm{~nm}$. Once the geometry of the molecule is reorganised after an electron loss, the predicted emission of the radical is calculated at $596 \mathrm{~nm}$. Keeping in mind that the calculated values are slightly different from the ones, obtained experimentally, we can still observe a large bathochromic shift and a red-shifted emission.

We propose that the DHICA in its phenolate form experienced ultrafast PCET that happens within the laser pulse. In sequential PCET model in DHI [127] proton transfer follows the transfer of an electron to the solvent and formation of solvated electron [133]. If we assume that this process happens in DHICA carboxylate anion, the electron transfer will turn DHICA into a radical and the following proton transfer will create a dianion radical, same as the product of the DHICA dianion. This process is illustrated in the central panel of Figure 3.19. However, if this process took place in DHICA in a neutral environment, electron transfer to the solvent 


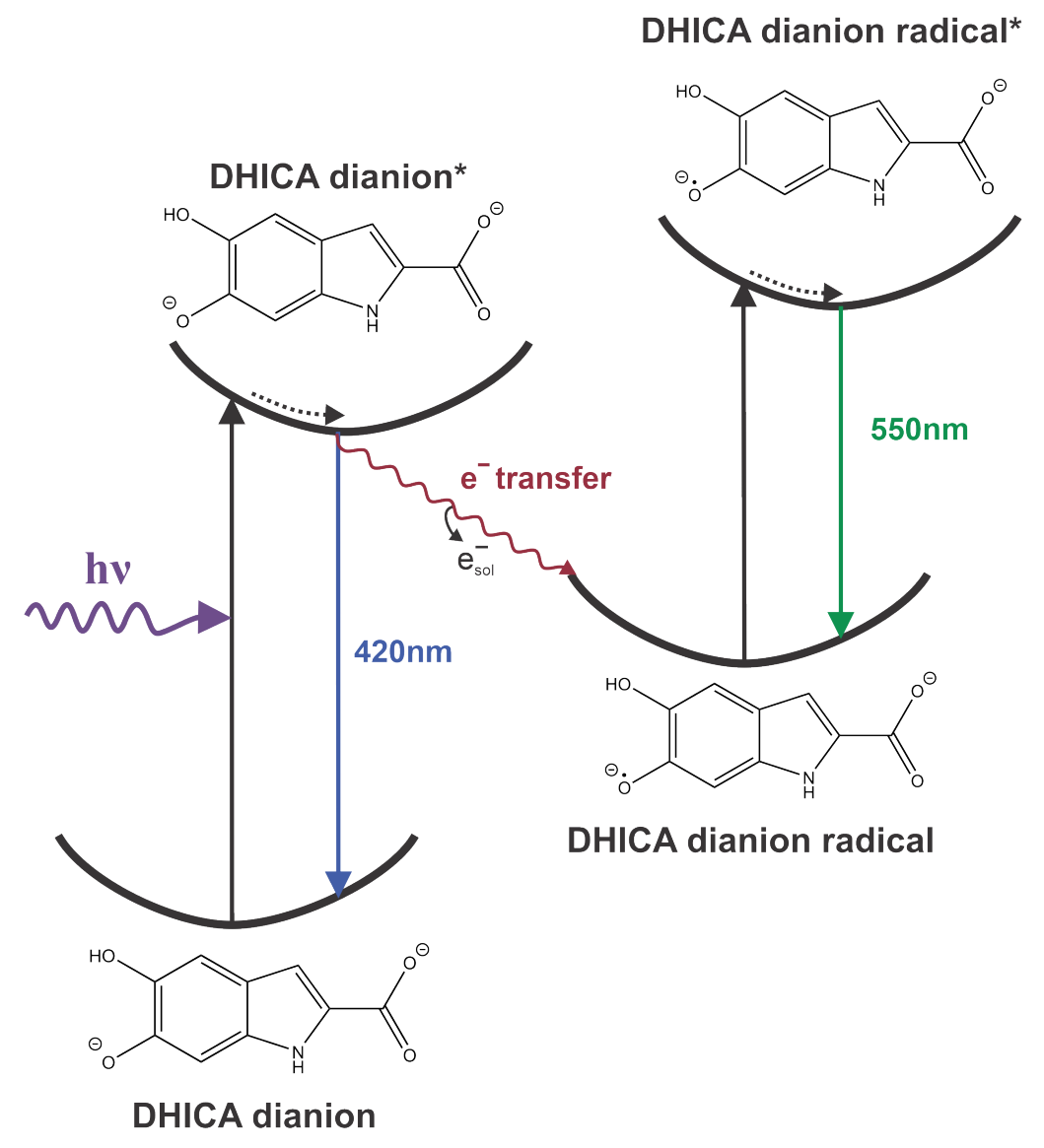

Figure 3.17: Proposed potential energy surfaces of the formation of the dianion radical.

(a)

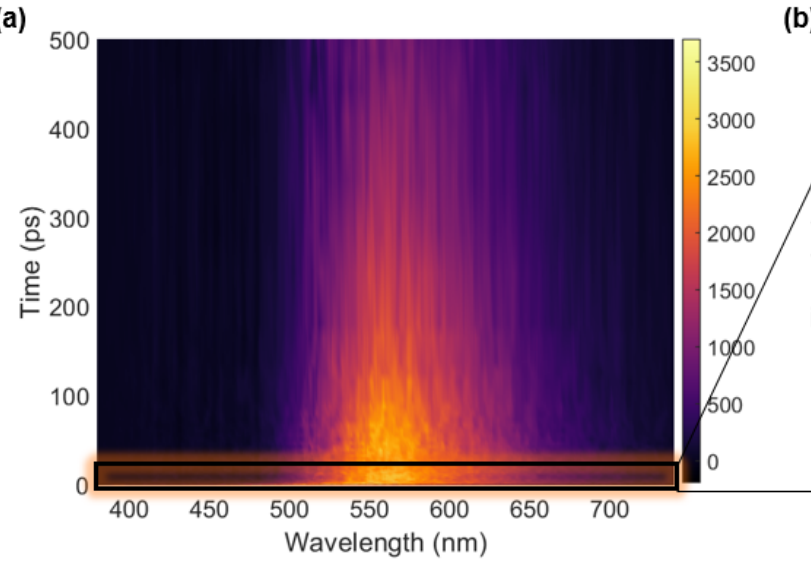

(b)

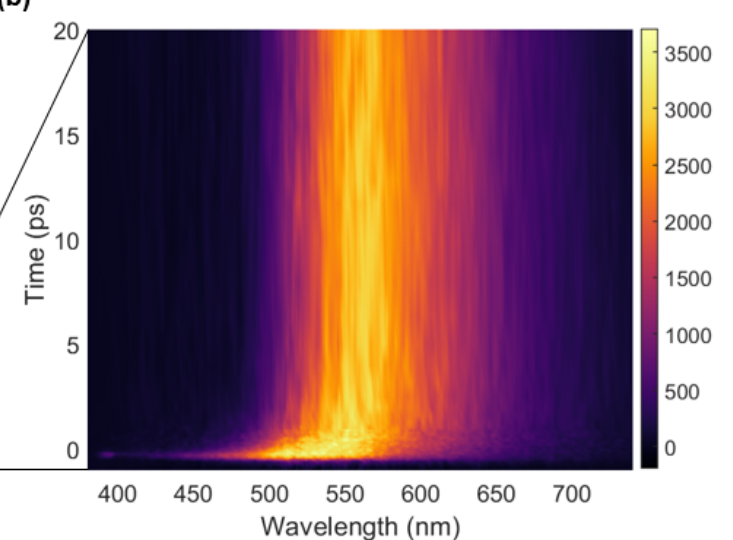

Figure 3.18: TRPL surface of DHICA in basic water, excited at the highest fluence $86.81 \mu \mathrm{J} / \mathrm{cm}^{2}$ : full surface (a), and first 20 ps after excitation (b). 


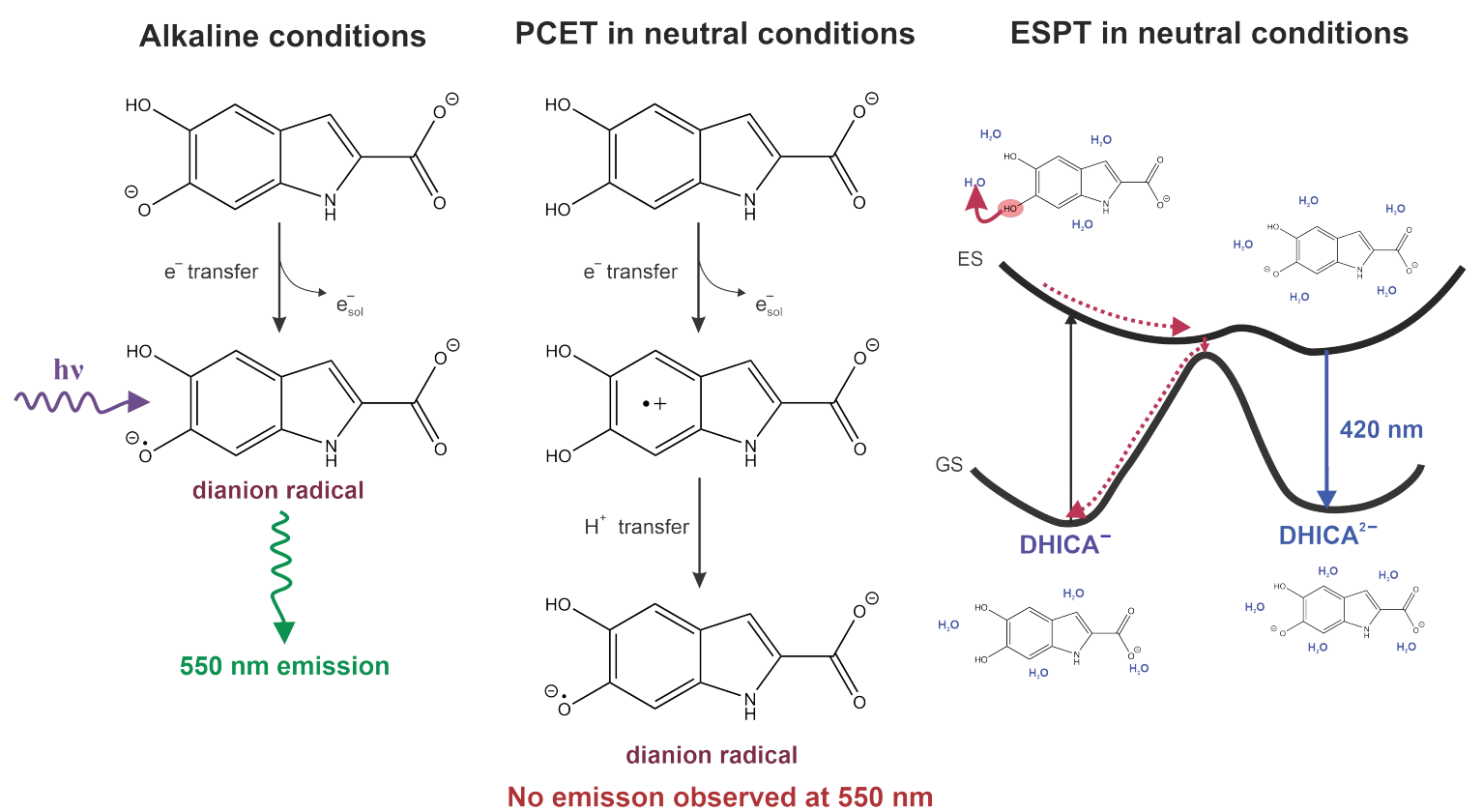

Figure 3.19: Scheme of the proposed photoproduct formation in alkaline conditions and the comparison of the PCET and ESPT processes in neutral conditions.

and the following proton transfer should happen within the pulse. Additionally, we would detect dianion radical emission at $\sim 550 \mathrm{~nm}$, like the one observed in DHICA in alkaline conditions. We do not detect any additional peak in either steady-state or time-resolved PL measurements at any fluence. Therefore we conclude that the ultrafast sequential PCET process does not take place in DHICA molecules.

Assuming that the electron transfer is fast enough for the sample re-excitation within the pulse, we have shown the dianion radical formation in DHICA in an alkaline solution. The same radical that would have been the result of the fast PCET process in water is not observed at any fluences used in the experiments. Therefore we conclude that the main excited state deactivation mechanism remains to be the ESPT from $\mathrm{OH}$ groups to the surrounding solvent.

\subsubsection{Role of the oxygen in the spectroscopic measurements}

We proved that DHICA monomer in water undergoes a few picoseconds excited state deactivation, as opposed to 1.6 ns decay, reported previously [38]. Such dis- 
crepancy between the results made us compare the experimental conditions of our measurements and those already published. The main difference was the sample preparation procedure - while we degassed all the solvents prior to the spectroscopic measurements, all the previously published experiments were conducted in aerated conditions. Therefore, it was important to address the role of the oxygen in the spectroscopic measurements and understand how does the presence of oxygen affect the lifetime of DHICA.

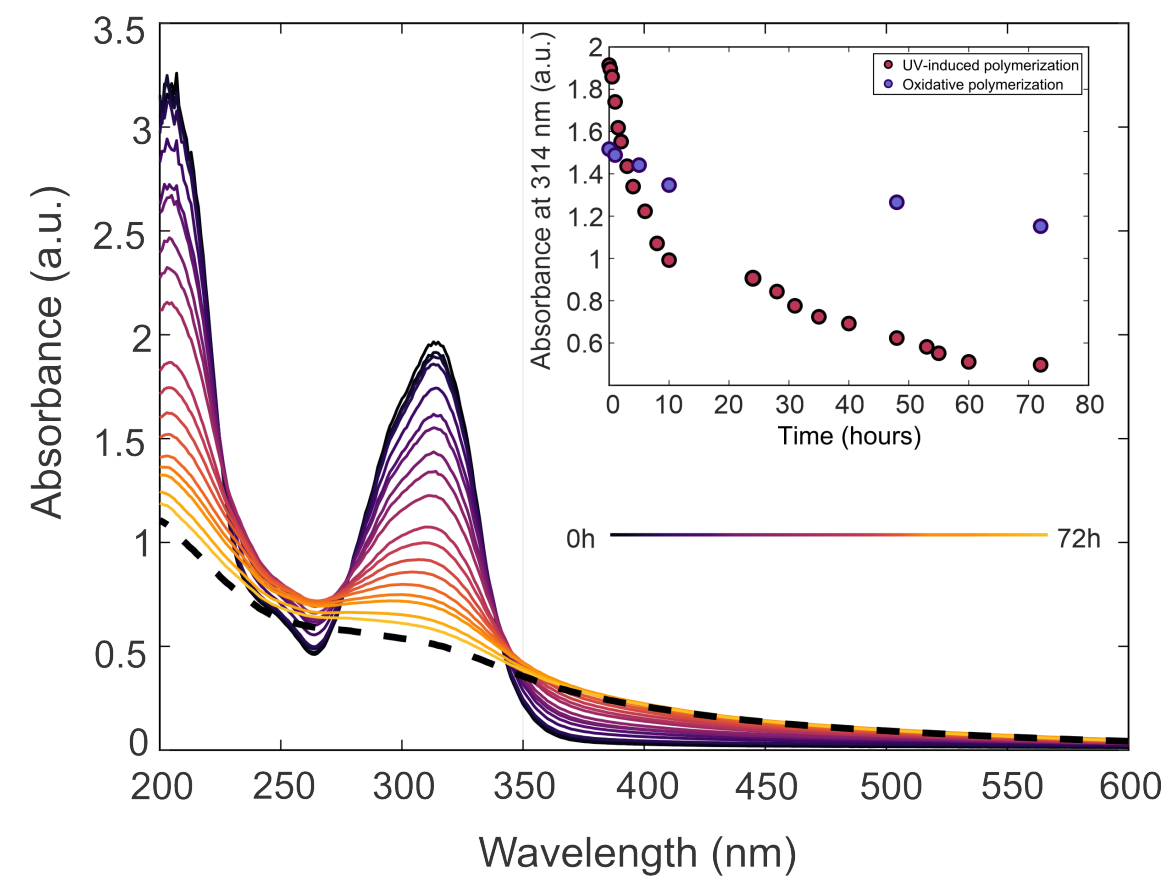

Figure 3.20: Absorption of DHICA monomer as it undergoes UV-induced oxidative polymerization in aerated water. The dashed curve shows the final absorption spectrum after $72 \mathrm{~h}$.

In aerated solution, DHICA experiences oxidative polymerization and evolves to form eumelanin-like aggregates. The process of steady-state absorption spectral evolution is shown in Figure 3.20. To collect a series of spectra of DHICA evolution into eumelanin-like form, DHICA was dissolved in MilliQ water, the sample was placed under weak UV laser radiation. For the first two hours the UV-visible absorption spectra of the sample were recorded every 0.5 hours; after that - every 2 hours on the first day, every 3 hours on the second day and third day. The final 


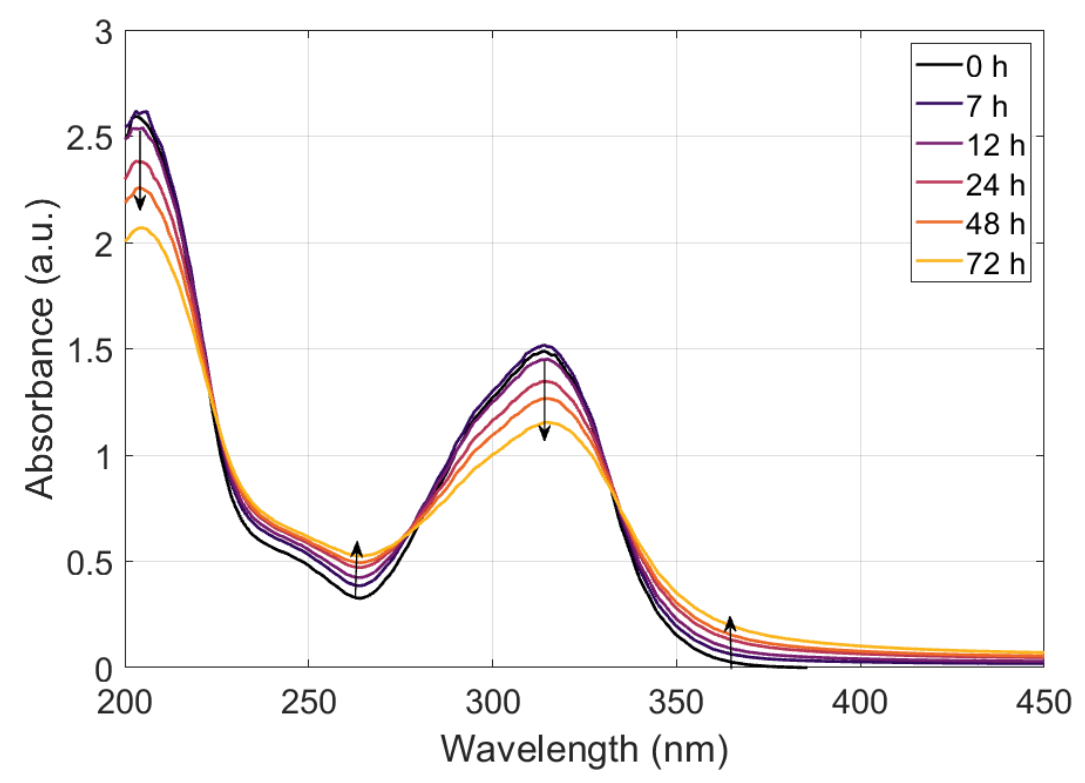

Figure 3.21: The changes in the steady-state absorption of DHICA monomer in the presence of oxygen in the dark.

spectrum (dashed black curve) was recorded on the fourth day, after 1 hour exposure to UV light. Without UV exposure DHICA was also evolving into the form of a eumelanin-like oligomer, but with a substantially lower pace (Figure 3.21). The reason for the increase of the polymerization rate might be the heating of the solution due to laser irradiation.

As DHICA undergoes oxidative polymerization (and forms dimers, trimers, and higher oligomers), the main peak experiences a slight red shift and broadens and flattens to form a eumelanin-like spectrum. This is caused by decreasing of the concentration of DHICA monomer. The newly formed species appear to have different excitation energies compared to DHICA monomer, so as their different absorption peaks overlap with each other, we observe spectral evolution into exponential-like shape, indicative of eumelanin.

To observe the difference between degassed and aerated conditions of the sample preparation, we perform transient absorption spectroscopy on DHICA in normal (aerated) water. TA is a very powerful tool to detect the formation of new species such as dimers or higher oligomers, that can be the result of oxidative polymeriza- 


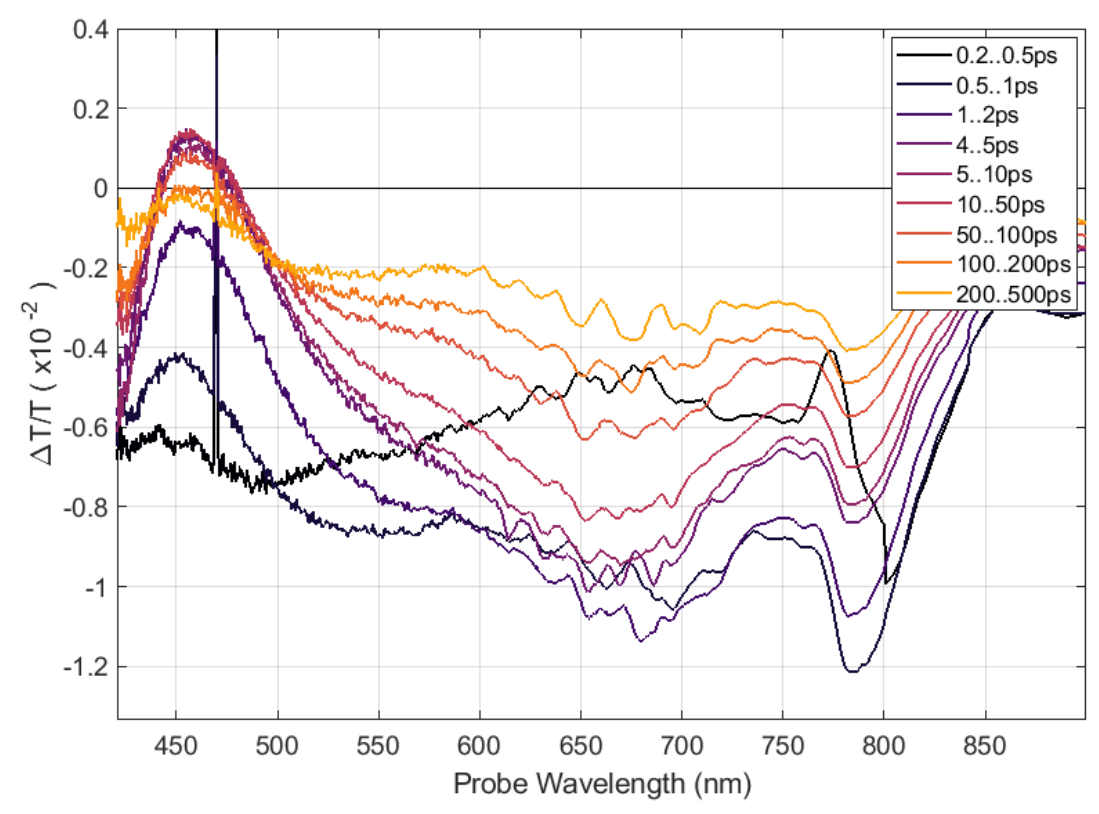

Figure 3.22: Evolution of transient absorption spectra of DHICA in aerated water.

tion.

For transient absorption measurements of DHICA building block, the monomer was dissolved in aerated MilliQ water at the same concentration as for the degassed measurements. The sample was excited at the third laser harmonics. We have also added the raster stage, translating the sample perpendicular to the excitation beam to try to prevent local polymerization during TA measurements, as we did for DHICA in degassed water. We could discover that after one TA measurement DHICA was experiencing polymerization as we could visually observe a formation of a dark-brown, melanin-like color strip where the excitation beam was moving inside the sample.

The extracted spectra are shown in Figure 3.22. By comparing these spectra with the monomer spectra, obtained in degassed water (presented in Section 3.2.3, Figure 3.8), we can see a drastic difference between the spectral shapes. At the first few femtoseconds after the excitation, DHICA maintains the monomer-like shape, showing the single PIA peak at $\sim 490 \mathrm{~nm}$. However, after that, we can observe that the said peak experiences the red shift to $\sim 500-550 \mathrm{~nm}$ alongside the formation of 
(a)

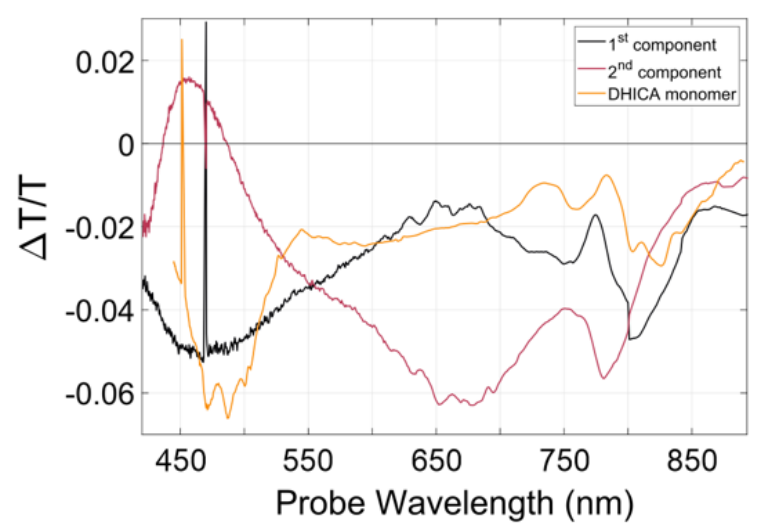

(b)

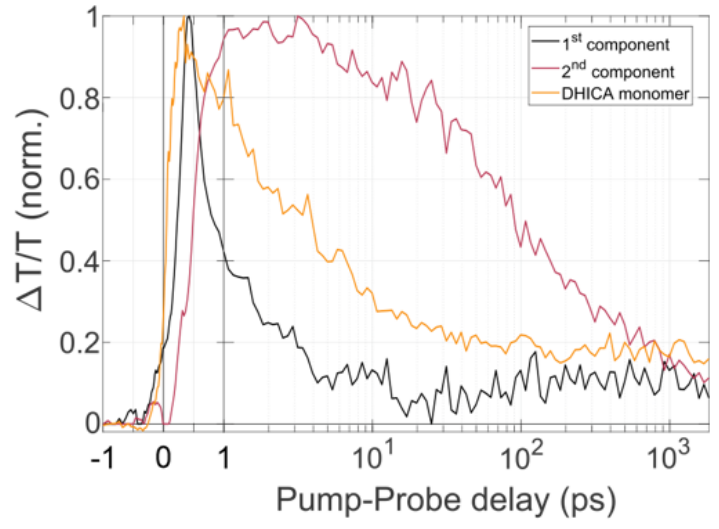

Figure 3.23: $M C R-A L S$ results of DHICA in aerated water.

a strong PIA band at $\sim 680 \mathrm{~nm}$. Together with the formation of the $680 \mathrm{~nm}$ band, we detect the GSB peak forming simultaneously at $\sim 460 \mathrm{~nm}$, indicating the formation of a new species. The long wavelengths data is not presented due to the laser's performance and a poor data quality.

(a)

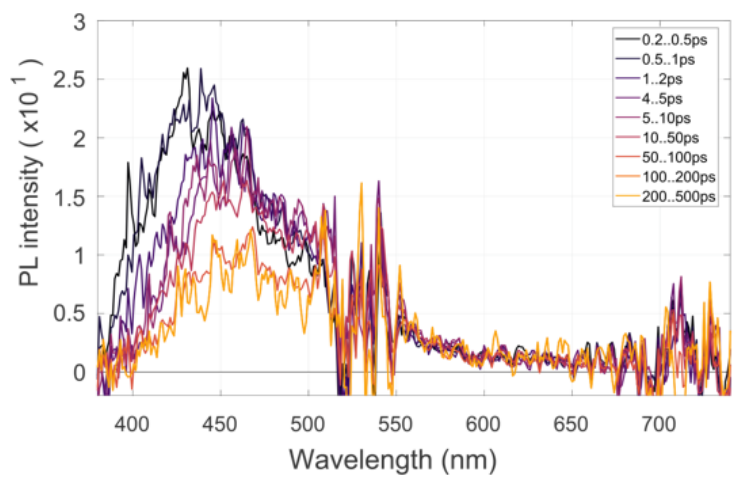

(b)

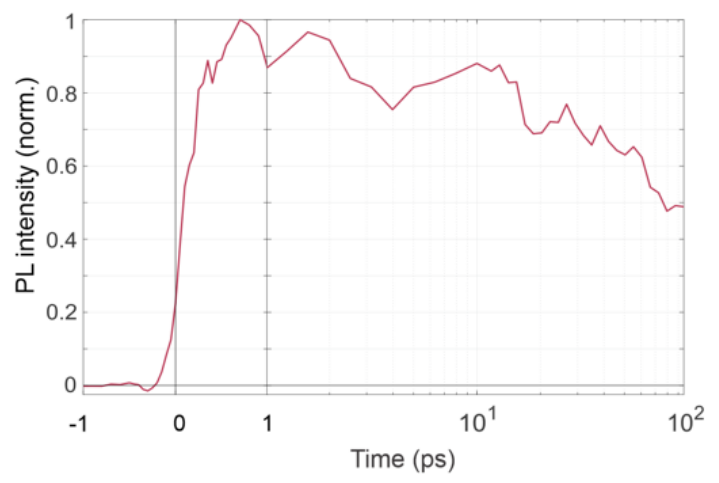

Figure 3.24: TRPL spectra (a) and kinetics (b) of DHICA in aerated water.

We separate two components of the spectra by using the MCR-ALS technique (Multivariate Curve Resolution-Alternating Least Squares 2.0 toolbox [83]). Spectra and kinetics of those components and their comparison with the real DHICA monomer results are presented in Figure 3.23. The first component showed, as pre- 
dicted, a spectral shape similar to that of DHICA monomer in degassed water. It only possesses one peak at $470 \mathrm{~nm}$ (the peaks in $800 \mathrm{~nm}$ are coming from the supercontinuum shape and the $800 \mathrm{~nm}$ fundamental contribution). The second component, possessing both GSB and PIA features, has a shape that looks more like a eumelanin spectrum (which will be studied in Chapter 5).

A positive GSB peak indicates a formation of a photoproduct due to the oxidative UV-induced polymerization of DHICA monomer. The oxidative degradation is also presented in the red shifting and broadening of the main PIA peak. As shown in Figure 3.23(b), the second component, appearing as a result of oxidative polymerization, has a longer lifetime than the first component's and the actual monomer's lifetime. This long decay is consistent with the long excited state lifetime, published before [38].

(a)

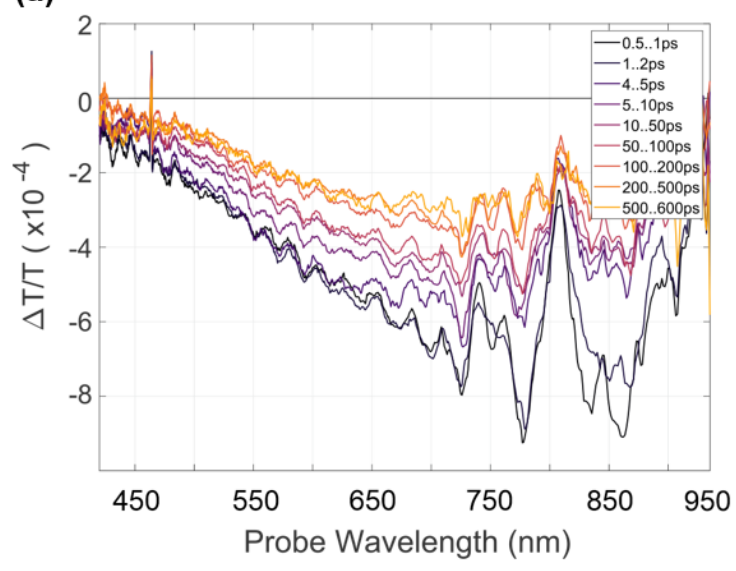

(b)

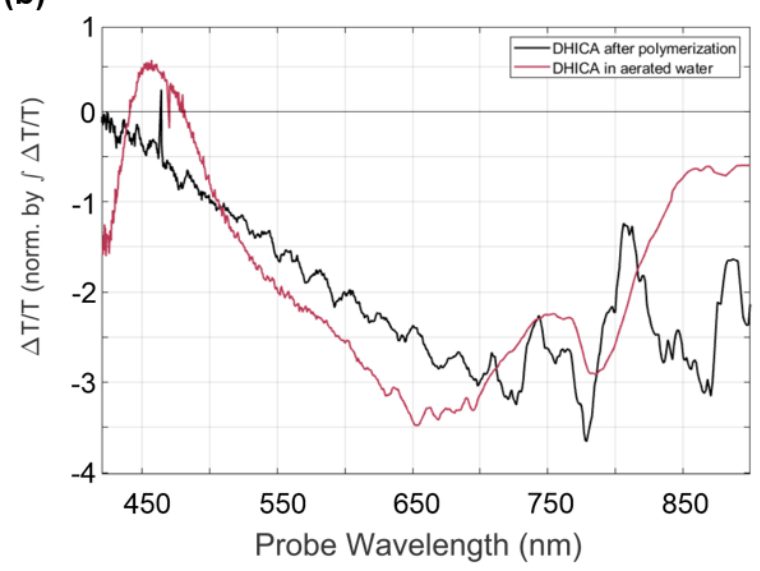

Figure 3.25: TA spectra of DHICA that went thought the oxidative polymerization (a); comparison of the spectral shapes of DHICA at the different stages of polymerization (b).

In TRPL measurements (Figure 3.24) the emission peak is slightly red shifted compared to the one, observed in DHICA in degassed water (presented in Section 3.2.3, Figure 3.11). By the brief examination, we can observe a considerable difference between degassed and aerated measurements, taken under similar experimental conditions. The kinetics of DHICA in aerated water exhibits a very slow excited-state decay in nanosecond timescale. This is fairly consistent with the pre- 
viously observed excited state lifetime of $1.6 \mathrm{~ns}$ [38]. The reasons for such a drastic increase of the excited state lifetime will be studied in Chapter 4.

Transient absorption spectroscopy has also been performed on the DHICA sample that went through oxidative polymerization, depicted in Figure 3.21. We compared it with the DHICA at the earlier stage of polymerization, i.e. DHICA monomer in aerated water (Figure 3.22). The results are presented in Figure 3.25. There are few differences between the spectra of DHICA in the early stage of polymerization and spectra of the DHICA in the later stage of polymerization. As we see in Figure 3.25 (b), depicting the late-time spectral shapes (50 ps), the PIA peak experiences a further red shift as the polymerization process in going further (strong artifact at 750-850 $\mathrm{nm}$ accounts for white light fluctuations in that spectral range and therefore this region of the spectra accounts to the spectral artifacts). However, for DHICA in the early stage of polymerization the main PIA peak is clearly positioned at $\sim 650 \mathrm{~nm}$, while for DHICA in the later stage of polymerization, there is a further progression of this PIA shoulder to NIR spectral region. Secondly, for DHICA in the late stage of polymerization we do not see any monomer indicating peaks around that spectral region. Additionally, UV-induced polymerization in water during a spectroscopic measurement is reflected in a photoproduct formation (late-time GSB peak). For a polymer, no GSB peak appears at any time of the experiment. So it can be concluded, that as much as DHICA steady-state absorption spectrum evolves to a form of eumelanin, the polymerized DHICA transient absorption spectral shape undergoes certain structural changes as well until it resembles the form of eumelanin.

The comparison of the excited-state kinetics, shown in Figure 3.26, demonstrates a long decay of DHICA in aerated water compared to decay of DHICA monomer in degassed water for both TA and TRPL results. In the same way, when looking at the TA kinetics for the DHICA in polymerized form, the decay of the main PIA peak is even slower than for the DHICA in aerated water, which turns into a polymerization photoproduct during the spectroscopic measurement. Thus, it is concluded that the excited state dynamics of DHICA monomer exhibits a substantially faster excitedstate decay than suggested in the literature. 
(a)

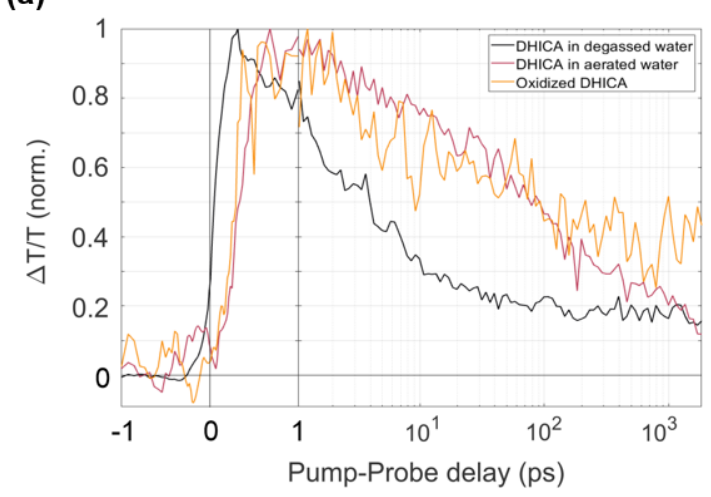

(b)

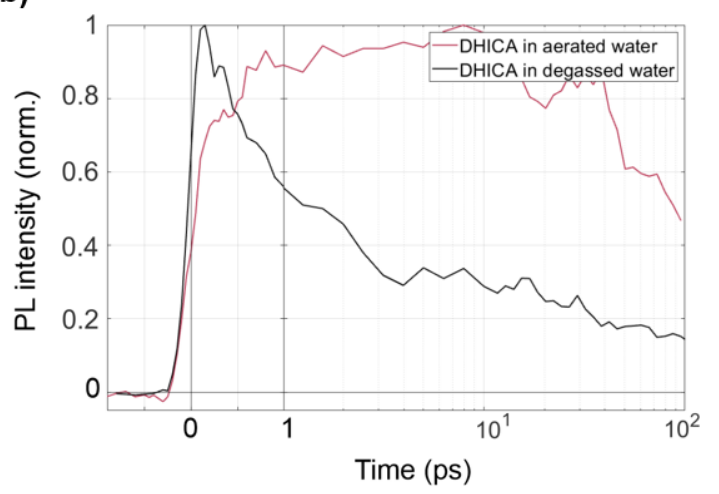

Figure 3.26: TA (a) and TRPL (b) kinetics of DHICA in degassed and aerated water.

Low fluence measurements of DHICA in aerated water (up to $40 \mu \mathrm{J} / \mathrm{cm}^{2}$ ), even without a visible formation of polymeric species on the cuvette wall, still led to the result, similar to one shown in Figure 3.22. Therefore, we concluded that degassing of the sample is extremely important in order not to compromise DHICA monomer measurements. This way the long lifetimes, obtained in the previous measurements [38] are actually the result of the uncontrolled polymerization of the DHICA samples, leading to a drastic increase of the excited state lifetimes.

This section demonstrates that oxidatively polymerized DHICA does not reflect the eumelanin pigment properties despite possessing a similar steady-state absorption spectral shape. The observed oligomer, unlike eumelanin, exhibits long excitedstate decay in order of nanoseconds, while eumelanin dissipates energy in the ultrafast picosecond time scale. It was previously suggested [37] that the oligomerization of DHICA results in the drastic shortening of the excited state lifetime while in fact the situation is reversed. The nature of the polymerized DHICA and the reason for the elongation of the excited-state lifetime is studied in Chapter 4.

\subsection{Ultrafast spectroscopy results of DHI}

DHI, as mentioned earlier, is a highly oxidizable molecule that tends to polymerize quickly in the presence of oxygen. Even when prepared under careful conditions, 
the dark, melanin-like impurities can contaminate the sample and create unwanted contributions to the time-resolved data.

For the studies of DHI monomers, DHI was purchased from Sigma Aldrich. The sample has a much darker brown color compared to related DHICA due to the present polymeric impurities. Therefore, care prior to and during the measurements was necessary. The samples were dissolved in water and methanol, that were degassed the same way as for the DHICA experiments. The sample degradation was monitored via UV-Vis steady-state absorption measurements. Polymeric impurities complicate sample preparation - they are highly insoluble in water even after the sonication that breaks some of the large aggregates apart. From that, DHI absorbance is substantially lower than that of DHICA even when using the same preparation methods.

From the steady-state absorption spectra, we can see that the available DHI samples have already started to experience oxidative degradation. It is reflected in the prolonged exponential-like "shoulder" after the initial peak. It is worth noting that the sample oxidative polymerization by the time of the first measurement has been caused by the long DHI compound shipping time (6 months) due to the COVID-19 outbreak and the related customs delays. Further oxidative degradation of the sample can be observed via steady-state absorption characteristics change (Figure 3.27). The progressing degradation is demonstrated in the blue shift of the main $S_{0}-S_{1}$ absorption peak and even lower DHI solubility in water. The comparison of spectral shapes revealed an even more exponential-like character of the absorption spectrum for aged DHI, which is more indicative of eumelanin.

Transient absorption measurements of two samples of DHI (Figure 3.27) were taken using the third laser harmonics as an excitation. The white light generation was performed with $\mathrm{CaF}_{2}$ crystal. The results, shown in the Figure 3.28 demonstrate two noticeable absorption bands at $500 \mathrm{~nm}$ and $680 \mathrm{~nm}$.

It was expected, in analogy with the DHI derivative DHICA, that the main absorption band of DHI will be in the region of 450-500 nm. The early TA measurements [61] have also reported the main absorption peak at $450 \mathrm{~nm}$ and the following band at $550 \mathrm{~nm}$. What is reflected in our TA measurements is slightly different as 


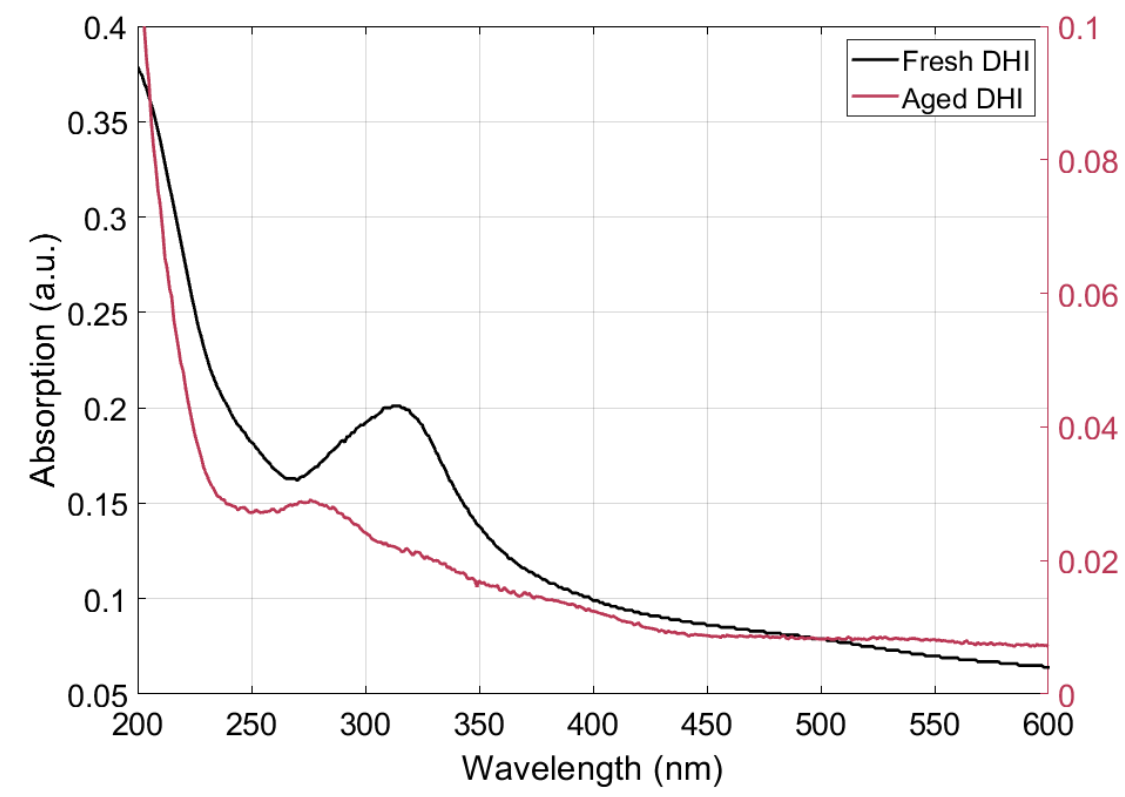

Figure 3.27: The comparison of $U V$-Vis of the newly made aged DHI sample a month after the first TA measurement (red) with the UV-Vis spectrum of the fresh DHI before the first TA measurement (black). 


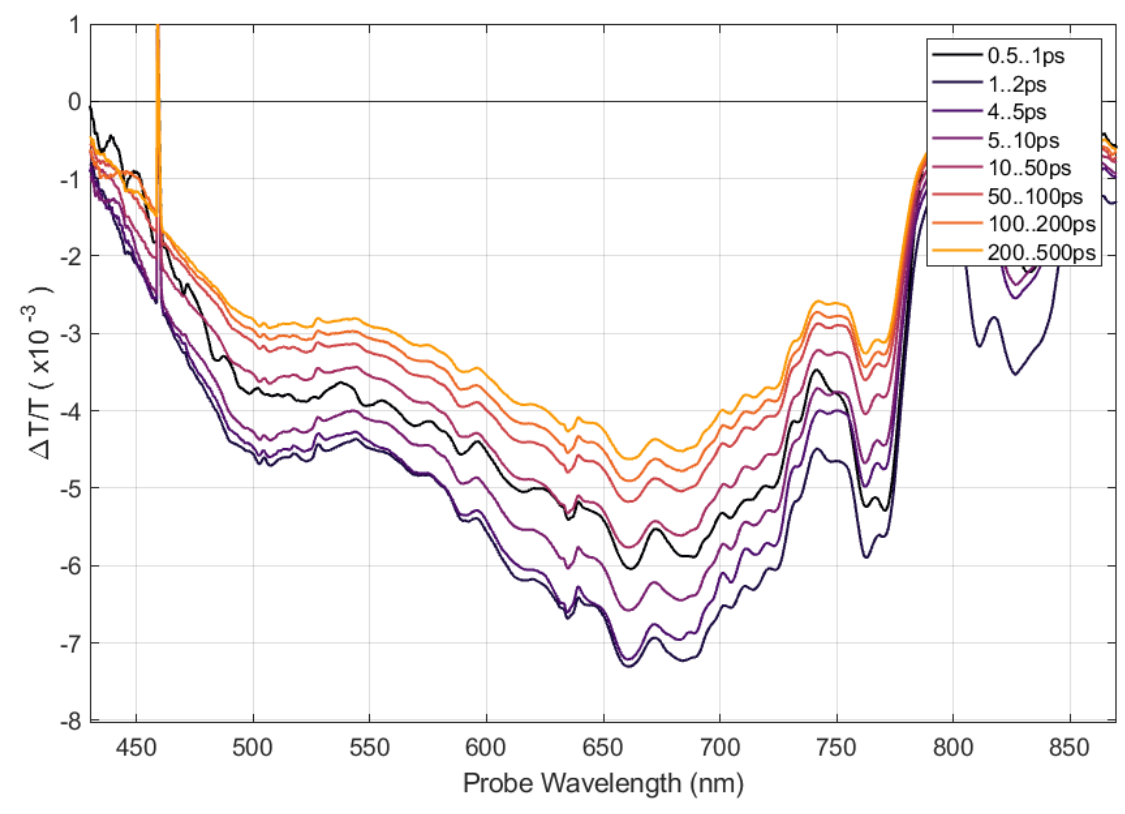

Figure 3.28: Transient absorption spectra of the fresh DHI sample.

a result of oxidative polymerization of the sample in the powder compound state. From the studies of the DHICA monomers, reported earlier in this chapter, we can conclude that the oxidative polymerization is reflected as a fast red shift of the main absorption band prior to the sample degradation.

To indicate the degree of the sample degradation we have performed another TA measurement on the significantly degraded DHI sample (Figure 3.27). Figure 3.29 shows a comparison for the two spectral shapes of both DHI samples recorded in similar experimental conditions. From the steady-state absorption results, it was expected that two TA spectra will be substantially different. In fact, the difference between them is fairly negligible. The first, and foremost observation is that the peak at $500 \mathrm{~nm}$ is lacking. That led to the hypothesis that the said peak, observed in the early measurement on the fresh DHI sample, is actually an intrinsic DHI monomer peak that might be red-shifted from the initial $450 \mathrm{~nm}$ value due to the pre-measurements degradation. The second peak at $\sim 680 \mathrm{~nm}$, observed in both spectra, is also different in the case of the aged sample, although the difference is almost insignificant. All we see in this case is a $\sim 30 \mathrm{~nm}$ red shift for the more degraded sample, as an 


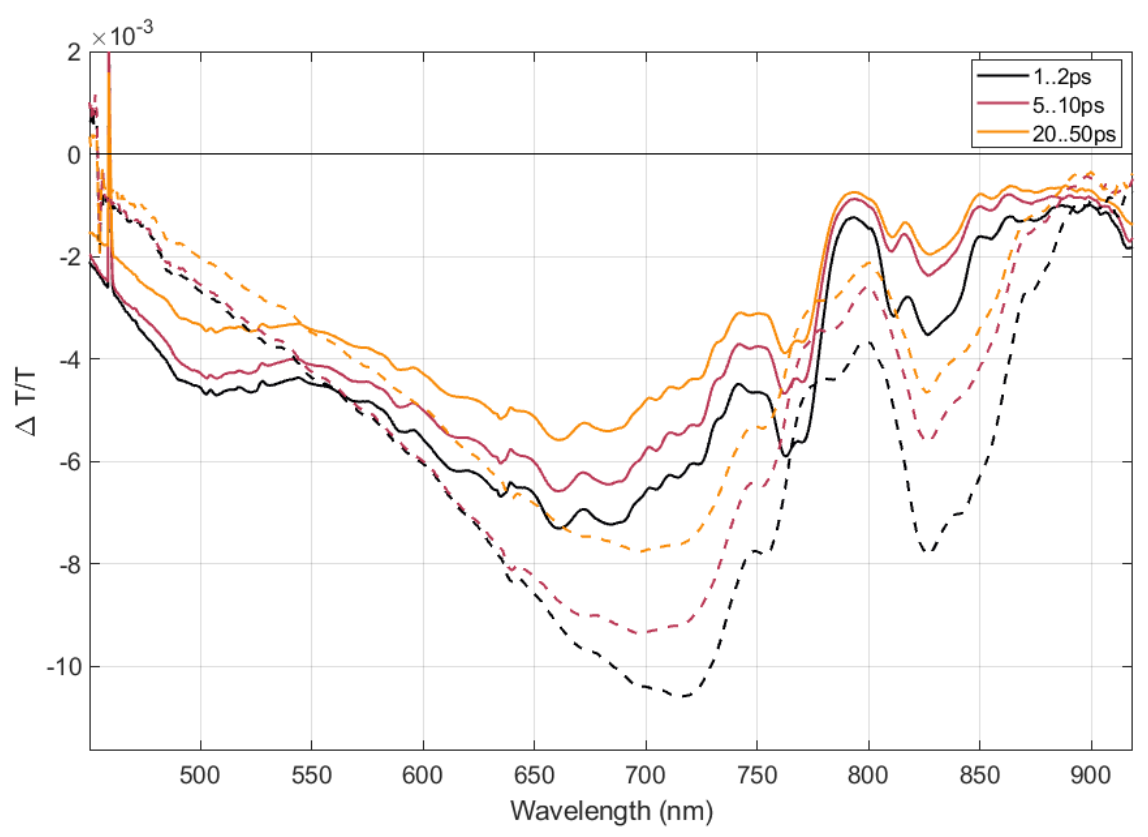

Figure 3.29: Comparison of transient absorption bands of the aged (dashed) and the fresh (solid) DHI sample.

account for the oxidative polymerization. It should be noted, for all spectral shapes, presented herein, the feature around $750-800 \mathrm{~nm}$ is appearing as a result of the white light spectral features related to the fundamental contribution.

The minor difference between the two DHI samples in seemingly different degrees of degradation proves that the sample, obtained originally from Sigma Aldrich has experienced an oxidative degradation, which indicates the inability of looking further into the ultrafast energy dissipation mechanisms of this building block and its role in the bigger picture for eumelanin pigment. Additionally, it was impossible to detect a TRPL signal in DHI samples due to their low solubility in water, resulting in the low optical density at the excitation wavelength. The large impurity aggregates appeared in the sample due to the degradation could also complicate TRPL measurements.

To study the role of proton-coupled electron transfer in DHI the freshly synthesized samples are needed to be measured in carefully prepared experimental conditions to prevent polymerization - for example, in addition to a necessary degassing 
of the solvent, the flow cell would be a useful addition, providing a fresh DHI sample exposure for each laser shot. This provides an opportunity for future research to expand the knowledge about the role of this key building block in eumelanin efficient photoprotection mechanism.

\subsection{Conclusion}

This chapter presents the ultrafast spectroscopy results for two key eumelanin building blocks DHICA and DHI. We have shown that DHICA in its monomer state loses roughly $\sim 1 \mathrm{eV}$ of energy in internal conversion. That leaves a lot of excess energy for proton transfer driving force, which was calculated to be $0.3 \mathrm{eV}$ for DHICA carboxylate. Solvent-dependent measurements revealed that DHICA monomer in neutral conditions exhibits an ultrafast excited state deactivation of $\sim 4$ ps, being in disagreement with the previously published 1.6 ns excited state lifetime. It was proven crucial to perform all spectroscopic measurements of DHICA and DHI monomers under oxygen-free conditions to prevent oxidative polymerization. Uncontrolled polymerization led to a drastic increase of the excited-state lifetime due to the formation of DHICA aggregates which will be studied in Chapter 4 . The prolongation of the excited state lifetime of DHICA monomer shows the importance of degassing the solvent before spectroscopic measurements that was not done before. Therefore, it was proved that the previously reported [38] excited state lifetime of $1.6 \mathrm{~ns}$ is incorrect.

The role of the ESIPT process in the DHICA monomer was proven unimportant, as the fully protonated form of the DHICA monomer was experiencing the same excited-state decay as the DHICA monoanion, incapable for ESIPT. The similarity of the two decays proves that despite the previous claim of the importance of the carboxylic acid group and its role in the proton transfer mechanism, the decay rate would not change upon deprotonation of the said group.

Strong evidence for ESPT as a primary relaxation pathway of DHICA monomers was presented by comparing the building block's decay in three key conditions - in neutral water, where proton transfer from phenol groups can occur; in basic water, 
where one of the phenol groups is deprotonated; and in methanol, where proton transfer is suppressed. It was found that the excited-state decay of DHICA in water is in picosecond timescale, which is consistent with proton transfer, and the kinetics trace for the excited state of DHICA in methanol and basic water both reflect a much slower decay. We demonstrated that, while the deprotonated form of DHICA in basic water exhibits a rather slow excited-state decay, in neutral conditions the amplitude of the ESPT from $\mathrm{OH}$ group allows the inter-surface transition to the ground state within a few picoseconds.

Using a combination of transient absorption and photoluminescence spectroscopy methods, we have detected the spectral signatures for DHICA monomers which will be used in the following chapters as benchmarks of studying eumelanin. DHI, notorious for being a strongly oxidizable molecule, was proved to be degraded over the time of the compound's shipping, making the study of its role in eumelanin pigment's ultrafast excited state deactivation nearly impossible, therefore it was not used in any further experiments in this project. 
- Chapter 4

\section{Role of oligomerization in DHICA energy dissipation}

\subsection{Chapter introduction}

Oxygenated polymerization of the DHICA monomers was proved to cause substantially longer excited-state decay rates than that of the pure DHICA monomers, measured in carefully prepared oxygen-free solutions. However, as we observed the formation of the eumelanin-like aggregates, the lifetime of the formed photoproduct was not consistent with eumelanin's role as the efficient photoprotectant. Therefore, the studies of the oligomers of DHICA and the difference between controlled and uncontrolled polymerization has become vital for understanding the role of DHICA building block in eumelanin photoprotective mechanism.

Oligomerization of the DHICA molecules was thought to lead to the drastic shortening of the excited-state lifetime [37]. It seemed to be consistent with the role of eumelanin as an effective photoprotector - in order for the pigment photoprotection to work, the large aggregates in eumelanin should dissipate the energy in an ultrafast manner. The excited state lifetime of the DHICA oligomers in the neutral aqueous environment has not been observed due to their low solubility. But, in analogue with acetonitrile measurements, the lifetime of DHICA trimer was predicted to be in order of tens to hundreds of picoseconds. Such a difference in the excitedstate lifetimes between monomers and higher oligomers led to a suggestion that the size and structure of the molecule influence the excited-state decay [60].

It was also suggested that large oligomers are more efficient in photo-protection than monomers and that the relaxation pathway depends on the size of the molecule and its structure [60]. The pH-dependent studies on DHICA oligomers revealed the decreasing of the fluorescence lifetime through oligomerization. Corani et al [37] studied DHICA dimers and trimers to observe a change in the excited state dy- 
namics as the molecule goes through oligomerization. According to their measurements, upon covalent bonding of DHICA monomers, the excited-state decay shortens from $1.6 \mathrm{~ns}$ to $300 \mathrm{fs}$. DHICA oligomers (starting with dimers) provide multiple functional groups with proton donor/acceptor properties, which leads to additional proton transfer pathways. That was believed to be a crucial point of the increased excited state deactivation efficiency on DHICA dimers compared to monomers. The authors have measured dimers in different conformational states, which exhibited similar dynamics, meaning that the mode of bonding does not play a role in the excited state deactivation process.

It was proposed that in the case of DHICA dimers and oligomers several phenol groups create a pre-existing hydrogen bond network, along which the protons can be transferred [37]. The proposed mechanism is illustrated in Figure 4.1. This network is claimed to be different in monomers, as covalent bonding was important for two or more DHICA oligomers and crucial for the rate of the proton transfer to the solvent. DHICA dimers and oligomers have the same solvent sensitivity as monomers - the hydrogen bond network, facilitating the proton transfer between $\mathrm{OH}$ groups and solvent molecules only exists in aqueous environments, while proton transfer is suppressed in methanol.

For identifying the minimal functional unit, responsible for ultrafast energy dissipation in eumelanin, DHICA-polymer was synthesized to compare its excitedstate decay with that of the dimer. In the previously published work [37], the comparison of the two forms have shown a very similar ultrafast sub-picosecond decay for both dimer and polymer. This was seen as evidence that the efficient excited state deactivation process starts in dimer and does not evolve much through polymerization. From that, it was decided that DHICA dimer provides a minimal functional unit for very efficient UV energy dissipation in eumelanin.

The above studies, however, did not consider the role of oxidative polymerization in the excited state deactivation of the DHICA monomer and higher oligomers, and all the samples were measured in aerated conditions. Therefore, we need to repeat those measurements with the proper sample preparation to ensure that the samples do not degrade prior to and during the spectroscopic measurement. 


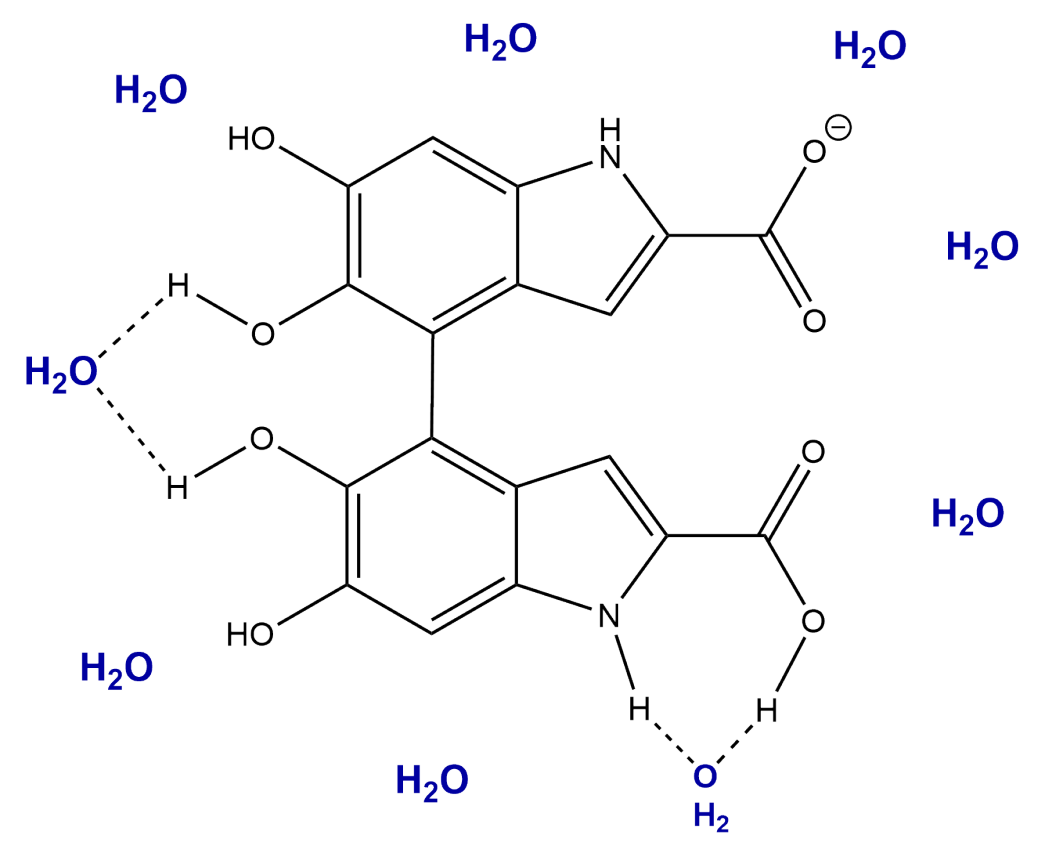

Figure 4.1: The proposed pathways for the ESPT in 4,4'-DHICA dimer.

In Chapter 3 we have shown the role of the solvent-mediated proton transfer from $\mathrm{OH}$ groups of DHICA monomer, as this is the main dissipation mechanism in the physiological conditions of the human body. The excited-state deactivation happened in the few picoseconds after the excitation, leading to a conclusion that the excited state lifetime of the monomers, published earlier [38], is inaccurate as the measured samples have degraded and formed a photoproduct, compromising the results. The observed uncontrolled oxidative polymerization, leading to a formation of an unknown photoproduct, results in a substantial increase of an excited state lifetime, rather than its shortening. Therefore, it is important to understand the role of oligomerization in the excited-state decay of DHICA as well as establish the minimal functional unit for eumelanin's energy dissipation.

In this chapter we will study the two types of polymerization of DHICA. Firstly, we will repeat the spectroscopic measurements of the synthesized DHICA dimer in degassed water to investigate the potential change in the excited state behaviour if we expand the DHICA building block from monomer to dimer. Secondly, to learn more about the role of the DHICA that went through uncontrolled polymeriza- 
tion, we will observe the compound in the different solvents in aerated conditions to unravel the discrepancy of the excited state lifetimes between formed DHICA oligomers and the eumelanin pigment.

\subsection{Sample preparation and steady-state characterisa- tion}

In this chapter, we observe two different samples. First, we have studied DHICA in phosphate buffer at $\mathrm{pH} 3$ in aerated conditions to see how does the uncontrolled polymerization affect the neutral form of the fully protonated DHICA and whether the polymerization process in neutral DHICA is different from DHICA anion. The monomer powder was dissolved in phosphate buffer with $\mathrm{pH}$. The carboxylic acid group's $p K_{a}$ is 4.25 so that in $\mathrm{pH} 395 \%$ of the DHICA molecules will be protonated.

After dissolving DHICA in the phosphate buffer, the samples were sonicated to break the large particles apart and then filtered through $0.2 \mu \mathrm{m}$ syringe filter. The final concentration of the sample was chosen to obtain 0.3-0.5 OD value at the wavelength of excitation (267 nm). The neutral DHICA in aerated $\mathrm{pH} 3$ phosphate buffer were then compared with DHICA in normal (aerated) MilliQ water (the results, presented in section 3.2.5. Figure 4.2 shows the steady-state absorption for two samples at different $\mathrm{pH}$. It is seen that upon deprotonation of the carboxylic acid group the maximum of the absorption spectrum experiences a blue shift.

Second, we performed transient absorption spectroscopy on the symmetrical 4,4'-DHICA dimer, shown in Figure 4.1, synthesised from the DHICA monomer by Dr Paul Hume of Victoria University of Wellington. Dimers were prepared according to the procedure, described in literature [134]. The isolation of the resulting product, high-performance liquid chromatography and ${ }^{1} \mathrm{H}-\mathrm{NMR}$ analysis, depicted in Figure 4.3 were performed by Sarah Andreassend of Victoria University of Wellington. The ${ }^{1} \mathrm{H}-\mathrm{NMR}$ peaks of $4,4^{\prime}$-dimer in $\mathrm{DMSO}-\mathrm{d} 6$ are assigned as follows: $\delta 6.31\left(1 \mathrm{H} \times 2, \mathrm{~s}, \mathrm{H}-3\right.$ and $\left.\mathrm{H}-3^{\prime}\right), \delta 6.87\left(1 \mathrm{H} \times 2, \mathrm{~s}, \mathrm{H}-7\right.$ and $\left.\mathrm{H}-7^{\prime}\right), \delta 11.14(1 \mathrm{H} \times 2$, bs, $\mathrm{NH}$ and $\left.\mathrm{NH}^{\prime}\right)$. A comparison of the steady-state absorption of DHICA monomer and dimer is shown in Figure 4.4 . 


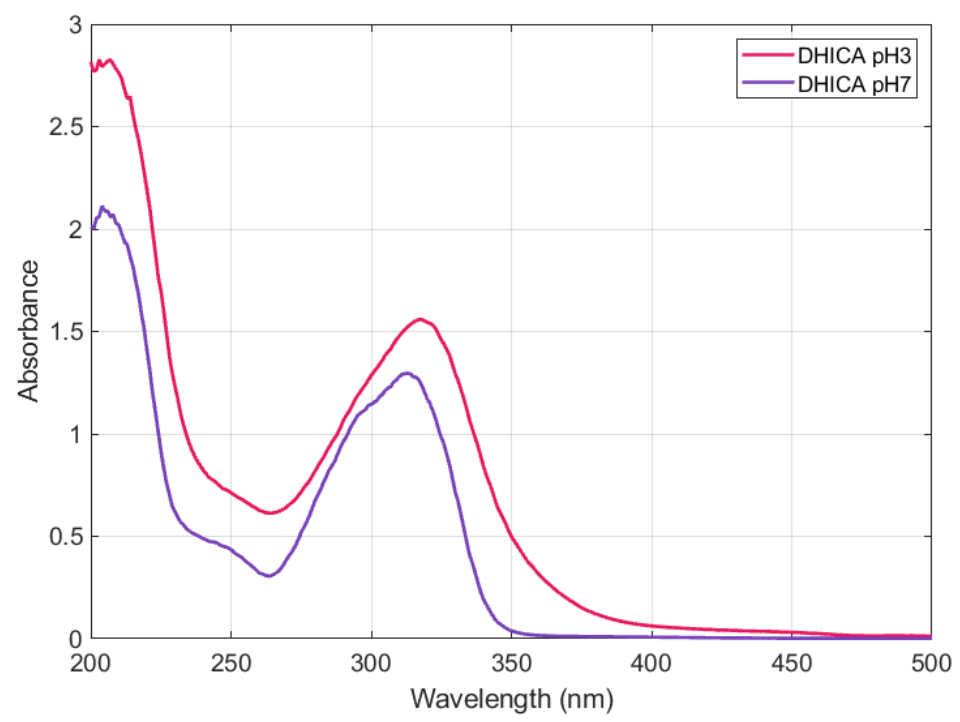

Figure 4.2: Steady-state absorption for two DHICA samples dissolved in phosphate buffers of different $\mathrm{pH}$.

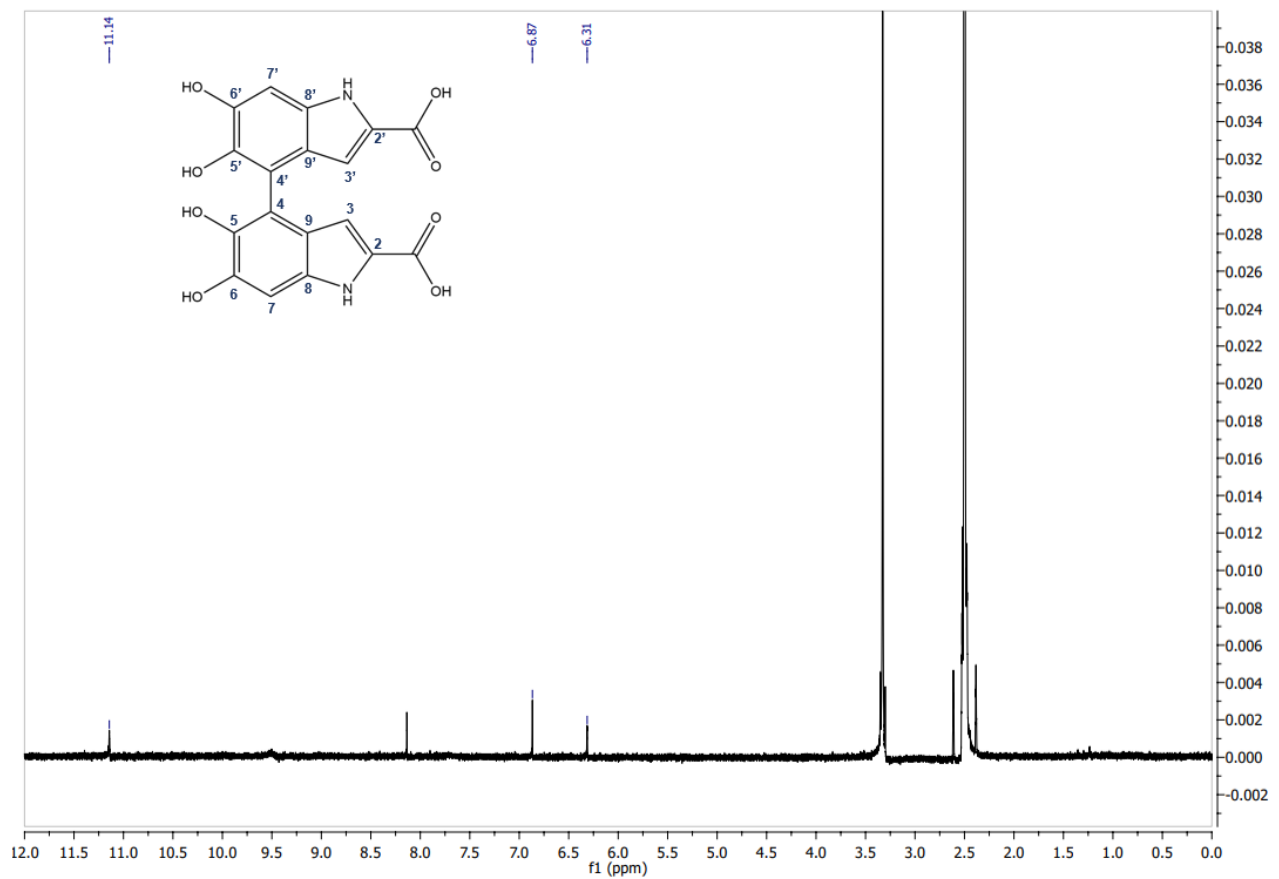

Figure 4.3: ${ }^{1} \mathrm{H}$-NMR spectrum of 4,4'-dimer in DMSO-d6. 


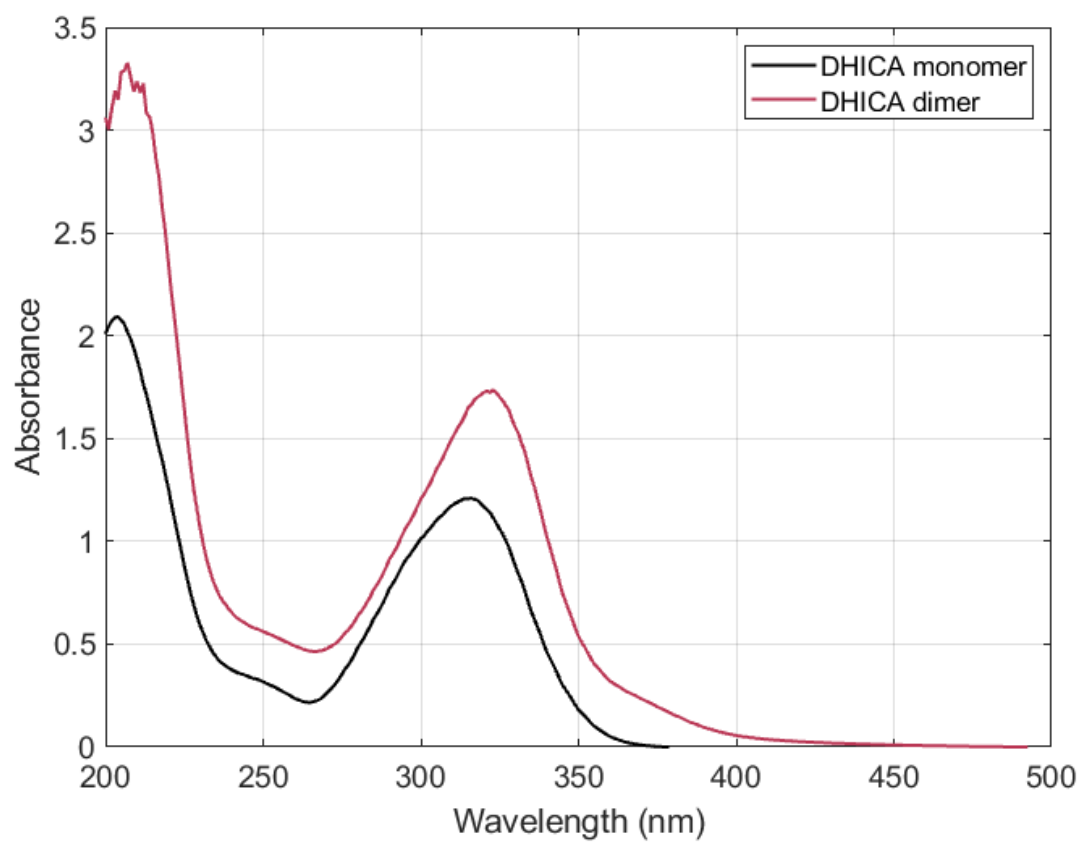

Figure 4.4: Steady-state absorption of DHICA monomer and dimer.

For TA measurements, dimer was dissolved in MilliQ water, which was previously degassed using the freeze-pump-thaw technique, described in detail in section 3.2.1. $3 \mathrm{mg}$ of dimer were dissolved in $1 \mathrm{~mL}$ of degassed water. After that, the sample was sonicated for 10 minutes. The resulting solution was slightly cloudy so to avoid laser scattering on undissolved particles, dimer solution was filtered using $0.2 \mu \mathrm{m}$ syringe filter.

\subsection{Experimental results}

\subsubsection{Transient absorption spectroscopy of DHICA dimer}

In Chapter 3 we have shown the role of the proton transfer from phenol groups to the solvent in the excited state deactivation of DHICA monomers. It is believed, that when we step up from a monomer to a higher oligomer, more ways for the proton transfer are opened, which would result in a drastic increase of the excited-state 
decay [37]. As was mentioned previously, DHICA dimer was thought to provide a minimal functional unit of the eumelanin ultrafast UV energy dissipation, which is the groundwork of the pigment photoprotective properties.

However, in the previous chapter, it was proved that DHICA monomer has a substantially faster decay than it was assigned for. The inaccuracy was originating in the way DHICA monomers were measured. Hence, the question about the minimal functional unit, responsible for eumelanin effective UV energy dissipation became open again.

For TA measurements of DHICA dimer, dissolved in degassed water, the sample was placed on the raster stage to prevent further polymerization. The sample was excited at third laser harmonics $(267 \mathrm{~nm})$, and for white light generation $\mathrm{CaF}_{2}$ crystal was chosen.

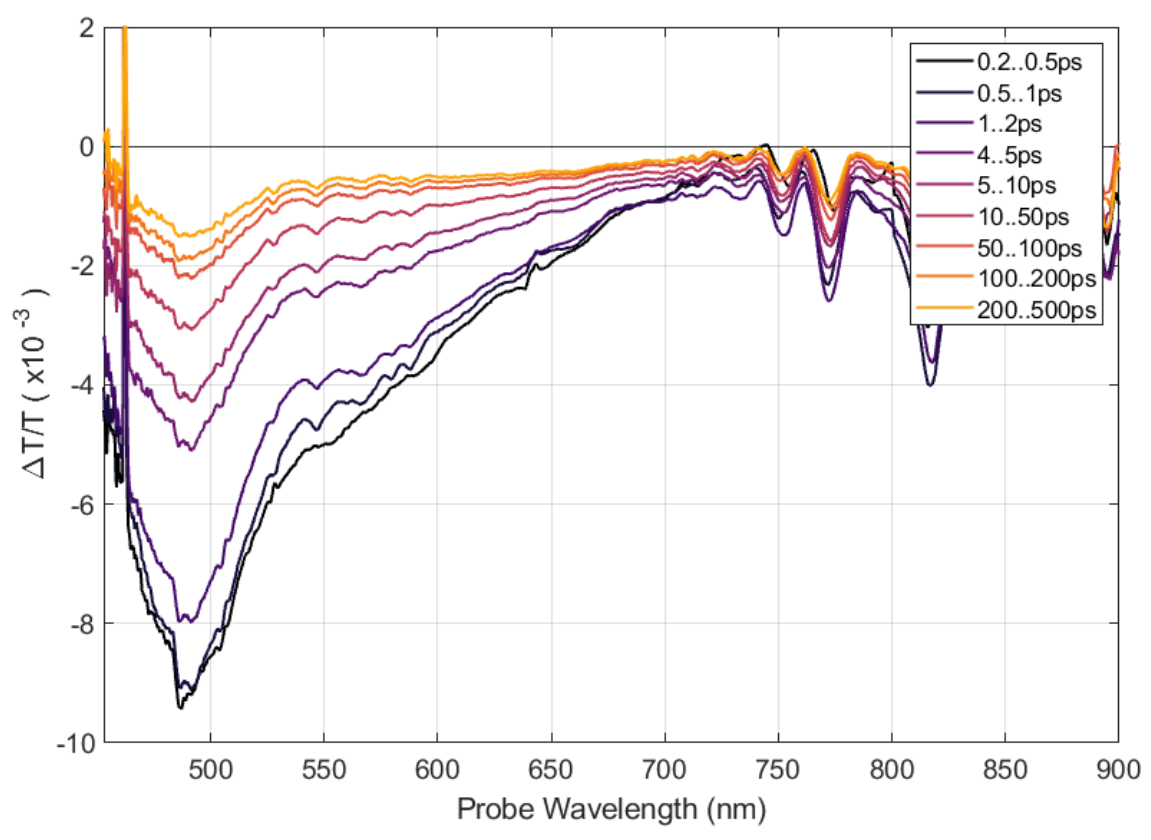

Figure 4.5: TA spectra of DHICA dimer in water.

Figure 4.5 presents transient absorption spectra of the dimer. It is clear that oxidative polymerization, which might have compromised the data, is not present, as we only observe a single PIA peak at $\sim 480 \mathrm{~nm}$, and this peak does not experience 

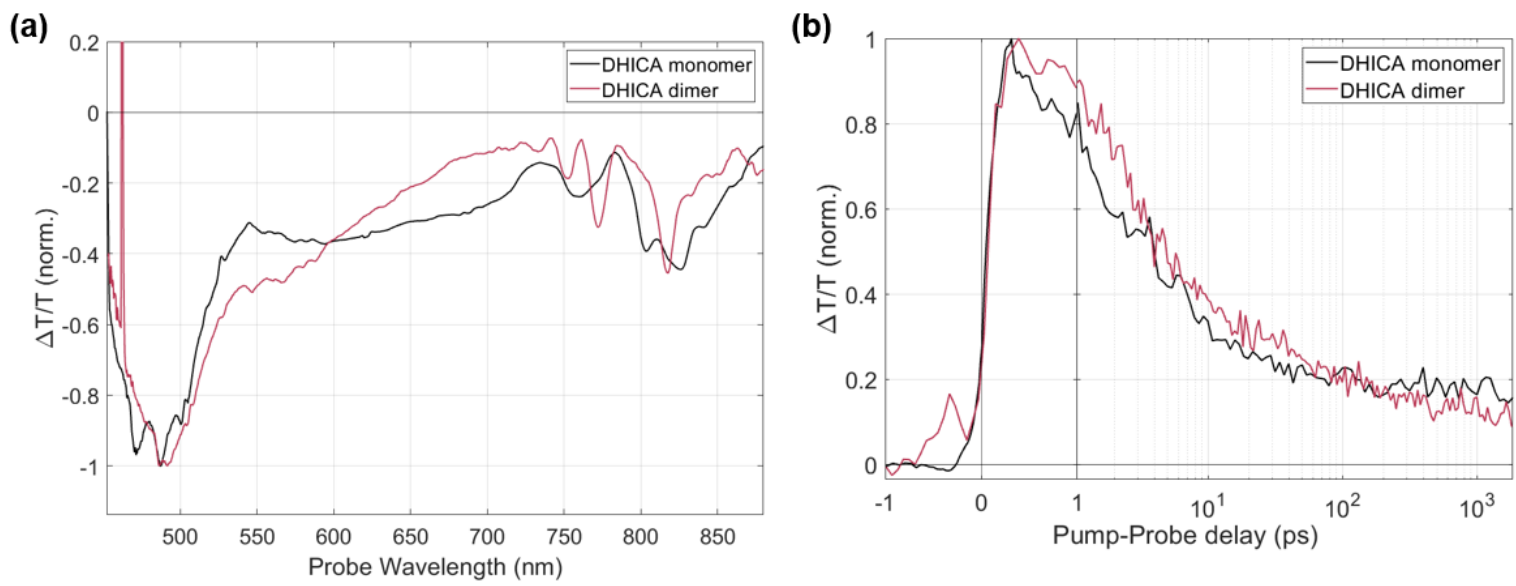

Figure 4.6: Comparison for TA spectra (a) and kinetics (b) of DHICA monomer and dimer.

a red shift over time, likewise, we do not monitor any photoproduct formation during the measurement. The main PIA peak, observed in the DHICA dimer looks very much similar to that of the monomer. Figure 4.6 shows a comparison for spectral shape and excited state kinetics for those two forms.

Two spectral shapes of monomer and dimer do not differ significantly. The position of the PIA peak, similar for both monomer and dimer, might serve as an explanation that the processes responsible for the excited state deactivation, are the same for DHICA in those forms of oligomerization. From the spectra, presented in Figure 4.6 (a) we observe no shift that would be indicative of a formation of a new state or a different excitation dissipation pathway. The absence of the PIA peak in the red spectral region (the one that was observed for DHICA in non-degassed water) implies that DHICA dimer has not experienced oxidative polymerization.

Kinetics traces are shown in Figure 4.6 (b). The comparison of the excited-state decays was expected to reveal a drastic shortening of an excited state lifetime of a dimer as compared to that of a monomer. Instead, not only we did not see the increase of a decay rate for the dimer, but the comparison has also shown that the two decays are similar. This does not match with what was observed in the previous research [37].

It was already mentioned in the previous chapter that careful sample preparation and removing the solvent's oxygen is crucial to avoid the oxygenation and further 
(a)

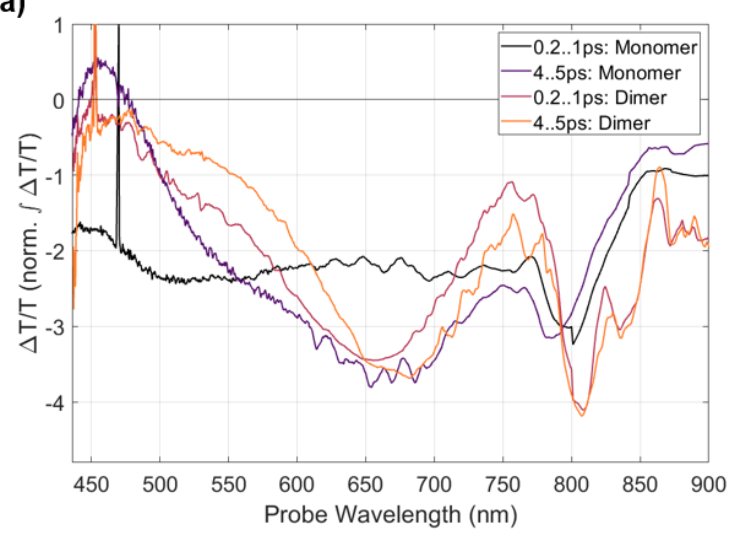

(b)

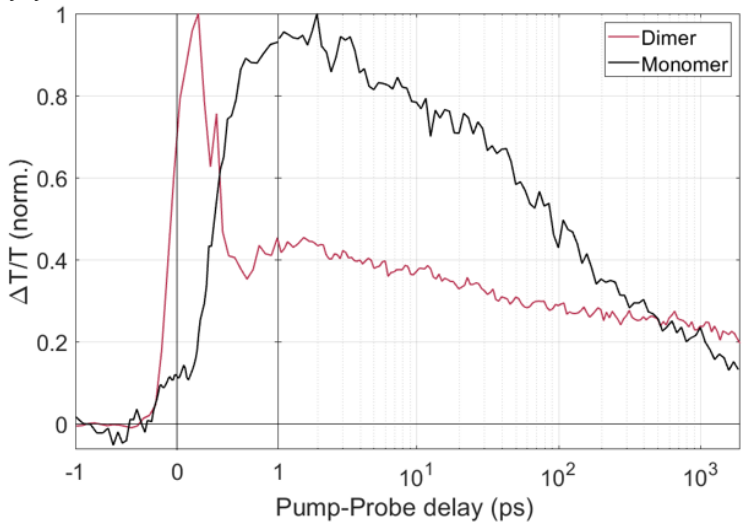

Figure 4.7: Comparison of TA spectra (a) and kinetics (b) of DHICA monomer and dimer, obtained in aerated conditions

polymerization of DHICA and DHI that will compromise the results. It should be also noted that we have shown that the monomer of DHICA possesses a fast excited state deactivation channel, which is much more effective than it was previously assumed - the excited state lifetime lies within a few picoseconds. The same decay, observed in the dimer, serves as evidence of the primary role of the monomer in excited state deactivation on eumelanin pigment.

However, we have shown that uncontrolled polymerization is reflected in the prolongation of the excited state lifetime. Keeping that in mind, the substantial shortening of a lifetime of DHICA dimer, measured by Corani et al. [37] seems inexplicable, especially because the experiments were conducted without degassing the solvents prior to the measurements. To test these previously obtained results, we have compared DHICA monomer (studied in Chapter 3, Section 3.2.5) and dimer, both dissolved in aerated water (Figure 4.7).

Red-shifted PIA peak at $\sim 680 \mathrm{~nm}$, that appeared in DHICA monomer in aerated conditions, is also present for dimer - it exhibits a small bathochromic shift in the first few picoseconds. Unlike the monomer that experiences GSB peak appearance which was assigned to a formation of an unknown photoproduct, dimer only possesses one PIA peak, matching that of a monomer. From the brief comparison of those two spectra, it is clear that the polymerization mechanism in monomer and 
dimer is different.

Kinetics traces, representing the excited-state decay of both monomer and dimer in aerated conditions, are shown in Figure 4.7 (b). We see that, like in the literature, monomer possessed a very long excited state lifetime, while the excited state of a dimer deactivates noticeably faster. We conclude that various polymerization mechanisms, observed in monomer and dimer in aerated conditions, are responsible for the difference in the excited-state decay of those two DHICA forms. That would also explain the drastic decrease of an excited state lifetime in DHICA dimer, observed by Corani et al. [37].

Considering the difference in the sample preparation method, performed in this project and the one used in the literature, it is again proven crucial to degas any solvent prior to a spectroscopic measurement. Taking into account that there was no substructure formation during the transient absorption experiment on DHICA dimer in degassed water, it is clear that the measured component was mostly a dimer, and there was no melanin-like aggregates formation. Hence, the excited-state decay, similar to that of a DHICA monomer, indicates that there is no increase in the excited state deactivation efficiency when we step up from a monomer to a dimer. To prove that the DHICA monomer is representing the minimal functional unit, responsible for the ultrafast energy deactivation in eumelanin, we have to compare DHICA monomer photophysics with that of eumelanin.

In future to have a full understanding of the different processes of oligomerization of DHICA and compare the controlled vs uncontrolled polymerization process we could synthesize the higher DHICA oligomers and measure them under the same degassed conditions as DHICA monomers and dimers and then compare with DHICA oligomers that underwent uncontrolled oxidative polymerization. An additional measurement for future research would be a preparation of DHICA-melanin as described previously [43] and the comparison of its excited state deactivation with that of eumelanin and DHICA monomer. 


\subsubsection{Effect of the uncontrolled polymerization on the DHICA excited- state decay}

The effect of the uncontrolled polymerization on DHICA molecules was discussed in Chapter 3, Section 3.2.5, and it was shown that under oxidative polymerization DHICA monomers go through oligomerization that results in longer excited-state decay. This was consistent with the previously published measurements [38], claiming that the DHICA monomers in water undergo slow excited-state decay. However, in acidic conditions, the excited state deactivation was reported to be fast despite being measured under aerated conditions. This indicates the difference between the formed oligomers and means that the conformational changes depend on the form of DHICA.

Additionally, one would expect that DHICA monomers go through oxidative polymerization and form eumelanin-like aggregates which would possess the excited state lifetime shorter or similar to that of a monomer. This unusual result required a further explanation. We hypothesise that the long excited state deactivation, observed in literature and in our experiments in aerated conditions hints that under aerated conditions DHICA forms the aggregates, incapable of proton transfer.

The inability to perform the excited state proton transfer to the solvent might originate in the high negative charge density formation when DHICA monoanion forms an oligomer. We assume that under neutral conditions when DHICA is a monoanion, the negative charge from deprotonated carboxylic acid groups creates a barrier for proton transfer. If this is correct, the situation should be completely different in acidic conditions, where DHICA is in its neutral form and therefore has no charge. In that case, the oxidative polymerization would not result in the long excited-state decay. For that, we compare the DHICA in aerated $\mathrm{pH} 3$ buffer with DHICA in aerated neutral conditions, that was presented in Chapter 3, Section 3.2.5.

Both forms of DHICA in acidic and neutral environments under aerated conditions experience the formation of a photoproduct at later times, which is reflected in the appearance of the GSB peak. Comparison of the spectral shape at early and late times is shown in Figure 4.8 (c). While it is nearly impossible to identify the 
(a)
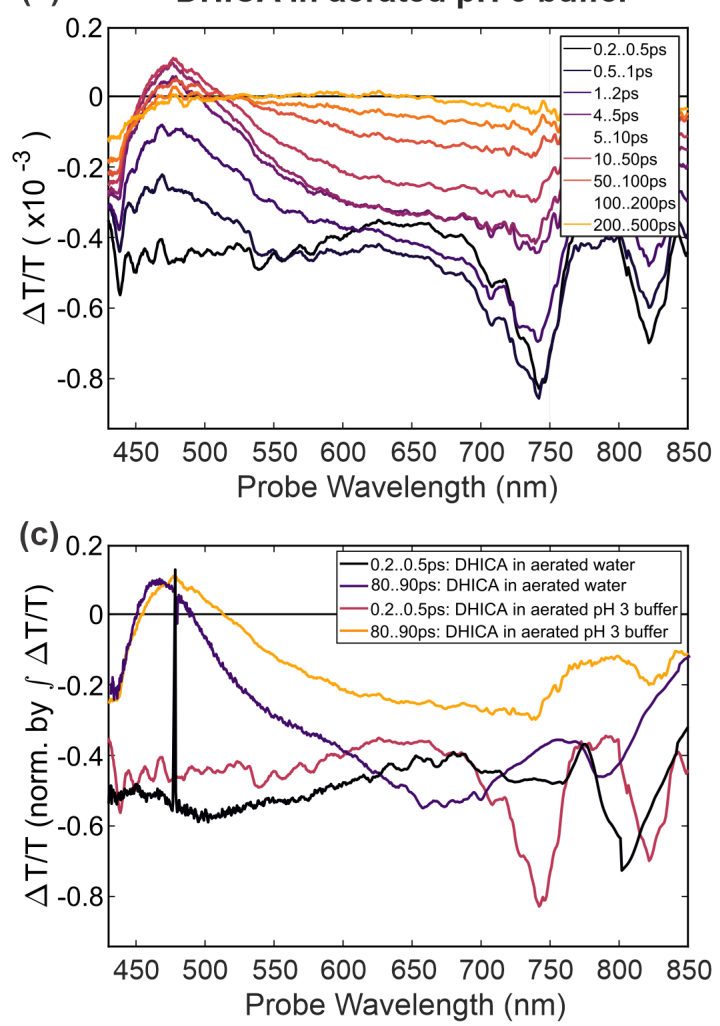

(b) DHICA in aerated water

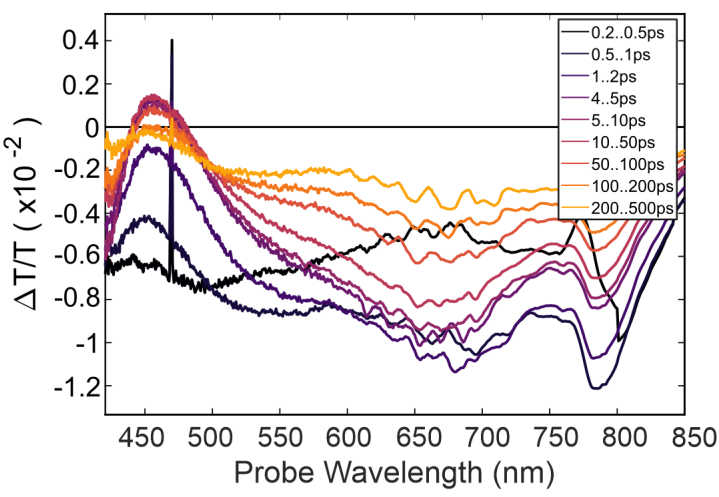

(d)

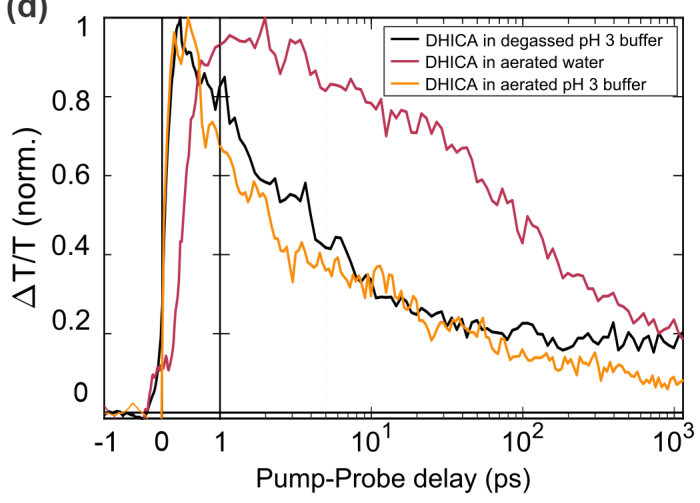

Figure 4.8: Spectral traces for DHICA in aerated pH 3 buffer (a) and aerated water (b), presented in Chapter 3, Section 3.2.5, Figure 3.22; comparison of their early-time and latertimes spectra (c) and excited state kinetics for the two oligomeric forms of DHICA with monomer (d).

peak of the early-time spectrum for DHICA in acidic conditions, we observe a red shift of the late-time spectrum as opposed to DHICA in neutral water. Specifically, that might be a reflection of the formation of a zwitterionic form, which is known to produce a red-shifted emission in the previous spectroscopic experiments.

The kinetics of neutral DHICA in an acidic environment is fast despite the oxidative polymerization. By comparing this decay with that of DHICA monomer in degassed pH 3 buffer (Figure 4.8 (d)), we can conclude that the decay rate for those two species in the different forms of oligomerization is the same. The excited state deactivation of the DHICA in aerated water is substantially slower. That already serves as evidence that the polymerization processes of DHICA in neutral and acidic 


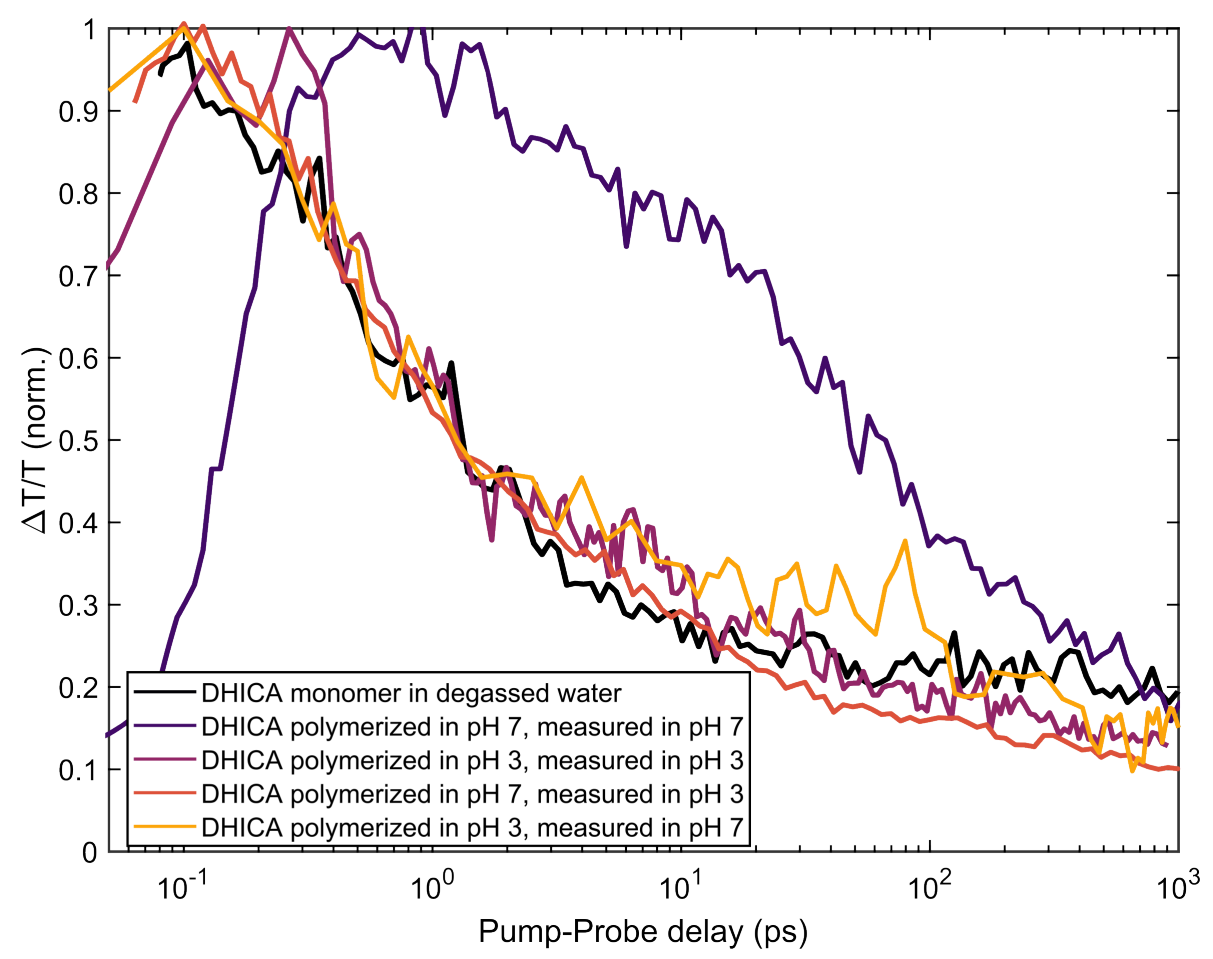

Figure 4.9: Comparison of the decays of different forms of DHICA, depending on the polymerization and measurement conditions. The obtained results were compared with the decay of DHICA monomer in degassed water.

conditions are not the same and under those conditions DHICA produces different oligomeric forms.

The discovery of the different oligomerization processes with and without the deprotonation of the carboxylic acid group and the negative charge formation motivated to decouple the polymerization conditions from measurement conditions. For the previous comparison, we studied oligomers that were measured under the same conditions in which they were polymerized. Since it was discovered that DHICA forms different aggregates in neutral and acidic conditions, by decoupling the polymerization from measurements conditions we will study the specific formations independently of the measurement conditions. For that, we prepared two other oligomers.

To obtain DHICA, polymerized in acidic conditions, and measured in neutral conditions, we performed a slightly different sample preparation approach. First, 
we oxidatively polymerized DHICA powder in aerated $\mathrm{pH} 3$ buffer, leaving it overnight and then added $\mathrm{NaOH}$ to the resulting oligomer to adjust $\mathrm{pH}$ to the neutral value. From the experiments on DHICA dimer, we know that under degassed conditions the formed oligomer would stay in the same state and would not go further through polymerization. Therefore, by degassing the resulting solution we ensure that the measured oligomer stays in the same formation as it was before degassing. The second oligomer was polymerized in neutral conditions and measured in acidic conditions. For that, DHICA was polymerized in water then $\mathrm{H}_{3} \mathrm{PO}_{4}$ acid was added to adjust the resulting $\mathrm{pH}$ to 3 and then degas the resulting solution.

The resulted comparison, shown in Figure 4.9, revealed that all the studied forms of DHICA, except the one, that was polymerized and measured in water, exhibit ultrafast excited-state decay, compared with that of DHICA monomer. The measured decay lifetimes are presented in Table 4.1. The fast decays are possessing similar lifetimes.

When DHICA is polymerized and measured in neutral conditions, it exhibits the longest excited-state decay. The possible explanation for this behaviour is that under the neutral conditions DHICA forms an aggregate that is not capable of proton transfer. The proposed reason for that was that in neutral conditions the high negative charge density creates the barrier that impedes proton transfer. Therefore, under acidic conditions, the created structure must be capable of proton transfer as the carboxylic acid group is protonated and there is no negative charge.

DHICA, polymerized in neutral conditions and measured in acidic conditions demonstrated ultrafast excited-state decay. We would expect to see a rather slow decay if the formed oligomer was possessing the form, incapable of the proton transfer. However, the explanation for this behaviour is that once we add acid, the carboxylic acid group becomes protonated and, therefore, capable of the proton transfer. Additionally, the protonation of the carboxylic acid group removes the negative charge and, therefore, the negative charge barrier.

The aggregates of DHICA, polymerized in acidic conditions and then measured in neutral conditions, must possess the alternative way of the structure conformation. Once the $\mathrm{NaOH}$ is added to the oligomer, formed in an acidic buffer, the car- 
Table 4.1: Lifetimes for the different DHICA polymerization and measurement conditions.

\begin{tabular}{||l|c|cc||}
\hline \multicolumn{2}{||l|}{} & \multicolumn{2}{c|}{ Polymerizarion } \\
\hline & & Acidic & Neutral \\
\hline Measu- & Acidic & $4.8 \mathrm{ps}$ & $3.9 \mathrm{ps}$ \\
rement & Neutral & $6.5 \mathrm{ps}$ & $500 \mathrm{ps}$ \\
\hline
\end{tabular}

boxylic acid becomes deprotonated. The fast degassing of the sample after adjusting its $\mathrm{pH}$ to the neutral value must have locked the structure in the formation where it is still capable of the proton transfer, unlike DHICA in neutral water.

The incorporation of quinone forms, described in Chapter 1, section 1.3.1, Figure 1.10 might also be altering the excited-state decay. These oxidized forms lack one or both protons in their phenol groups. Hence, once the proton transfer is removed as an option on quinone forms with oxidized $\mathrm{OH}$ groups, the decay might become slower, which is happening due to the oxidative polymerization in neutral water. The quinone forms might therefore alter the molecule conformation by locking it in the form, incapable of proton transfer. The investigation of the quinone forms incorporation might be an important piece of information lacking about the oxidative polymerization effect on the DHICA excited-state decay.

\subsection{Conclusion}

In this chapter we have considered the role of oligomerization, both controlled and uncontrolled, on the excited state deactivation of the key eumelanin building block DHICA. Transient absorption spectroscopy measurements were conducted for the symmetrical DHICA dimer, specifically synthesized for this experiment. The dimer was measured in both aerated and degassed conditions to have an idea about the differences between the pure dimer and polymerized dimer's spectroscopic signatures as well as the excited-state decay rates. We have found that, unlike monomer, DHICA dimer in aerated conditions possesses a much faster excited state deactivation mechanism, while it was expected to observe a long excited state lifetime due to an uncontrolled polymerization. Hence, we conclude that monomer and dimer 
exhibit different polymerization processes. Aerated conditions data is in agreement with the previously published claim that the dimer possesses a much efficient excited state deactivation mechanism than monomer and therefore it was believed that it presents a minimal functional unit, responsible for the effective photoprotection in eumelanin.

However, transient absorption measurements of a degassed sample demonstrated that dimer and monomer both experience similar decay, showing almost no difference in the observed kinetics rate. Keeping in mind the importance of degassing the sample prior to the measurement, as well as the absence of a red shift in the spectra, we conclude that, unlike it was claimed before, the DHICA dimer is not a minimal functional unit. Ultrafast character of the DHICA monomer's excited state deactivation and its similarity to that of a dimer make us come to the conclusion that the DHICA monomer might be the main energy dissipation unit in the pigment, yet further evidence of this claim is still needed.

Uncontrolled polymerization was shown to cause a significant decrease of the excited-state decay rate, as opposed to the assumption that the oligomerization of DHICA would turn it into an eumelanin-like molecule and would rather increase the excited-state decay rate. The reason for that was found to be a negative charge repulsion that creates a barrier for the proton transfer to the solvent and therefore slow down the excited state deactivation. This assumption was proved by comparing the polymerized DHICA in neutral and monoanion form since in an acidic environment DHICA is electroneutral and would not create the high negative charge density field. We showed that in an acidic environment, where the carboxylic acid group remains protonated, DHICA does not form an aggregate that would impede the proton transfer to the solvent, since it exhibits the fast excited-state decay, compared to that of DHICA monomer. This again emphasizes the important role of the DHICA monomer and implies that the monomer, and not dimer or higher oligomer, is representing eumelanin's minimal functional unit.

The study of the effect of oxidized forms of DHICA on excited-state decay might provide a further explanation of the role of uncontrolled oxidative polymerization on DHICA. The oxidation of one of two phenol groups can lead to the decrease of 
the excited-state decay rate due to removing ESPT as an option.

To understand the role of the DHI key building block in the excited state deactivation of eumelanin, the fresh DHI monomer can be used to prepare the various oligomers, containing different amounts of DHI and DHICA. We assume that DHI would mend the effect of oxidative polymerization of DHICA, which is shown to result in elongation of the excited state lifetime. The incorporation of DHI building block and DHICA/DHI quinones might affect the excited-state decay and produce oligomers, that presumably form a part of eumelanin pigment. The study of those structures and the excited-state decay dependence on the DHI/DHICA ratio in the synthesized oligomers will close the remaining knowledge gap about the role of each of the building blocks in the UV energy dissipation in eumelanin pigment. 
- Chapter 5

\section{Ultrafast spectroscopy of eumelanin pigment}

\subsection{Chapter introduction}

The excited state dissipation of eumelanin pigment that accounts for its efficient photoprotective properties, was extensively studied over the past decades $[22,92$, $124,126,147,183]$. The low photoluminescence quantum yield indicated the importance of the rapid nonradiative excited state deactivation channels. It was shown that the pigment possesses the radiationless ultrafast excited-state decay, however, the nature of this decay remained unknown.

Eumelanin is notorious for its high degree of disorder that complicates the study of the energy dissipation nature of the pigment. As was previously mentioned in Chapter 1, chemical and structural disorder can account for the broadband nature of eumelanin's absorption spectrum, showing that the broad shape of the spectrum is coming from the individual spectra of consistent oligomers, overlapping with each other (Chapter 1, Figure 1.12).

While the disorder plays an important role in eumelanin's optical properties, we seek to understand whether it accounts for the pigment's ultrafast energy dissipation. Due to the high degree of disorder in eumelanin, the chromophores can come very close to each other and create excitations that can then funnel through the chromophores so that the disorder might play a dynamic role in the excited state deactivation of eumelanin. Kohl et al. [92] performed the spectral hole burning spectroscopy on the synthetic eumelanin. They discovered that the GSB signal does not experience red shift over time, which would be an indication of the dynamic energy transfer between the chromophores. However, just the lack of spectral dynamics might not be enough to claim the unimportance of the role of chemical disorder in energy dissipation in eumelanin. The different GSB peaks, that were supposed to 
appear as the result of the dynamic energy transfer between chromophores, could be overlapping with the PIA peaks, cancelling each other out. Therefore it was crucial to conduct additional analysis to establish whether the excitations in eumelanin are mobile or the energy dissipation has a localized character.

The lack of the spectral dynamics in eumelanin led Kohl et al. [92] to the hypothesis that eumelanin aggregates are capable of creating immobile charge transfer states. This hypothesis was further supported by conducting the polarization anisotropy transient absorption measurements. However, the authors did not consider the role of the building blocks DHI and DHICA in the excited state deactivation. The polarization anisotropy might be an intrinsic property of the building blocks, that are unable to create a CT state, so therefore, this assumption is found to be incomplete.

The complexity of eumelanin was the reason for the used bottom-up approach to address the pigment's dynamics. The key building block DHICA monomers [38, 60] and oligomers [37] were specifically studied to reveal its dissipation mechanism and determine its importance for eumelanin's photophysics. It was thought that DHICA lifetime decreases dramatically as the molecule goes from monomer to dimer due to the formation of the intramolecular hydrogen bonds. Those hydrogen bonds facilitate proton transfer in DHICA, so it was postulated that the ESIPT and ESPT processes are taking place in DHICA. The authors have also claimed that the DHICA dimer represents the minimal functional unit that is responsible for eumelanin's photophysics.

In the previous chapters we showed that the previously published studies did not address the problem correctly as the authors did not take the DHICA oxidative polymerization into account. Our experiments demonstrated that, when prepared in an oxygen-free environment, DHICA molecules display a completely different behaviour to what was discovered before $[37,38,60]$. It was proven that the oxygen affects and compromises the data and that DHICA monomers are possessing an ultrafast energy dissipation character, similar to that of a dimer. Hence the question was about the role of DHICA monomer in eumelanin energy deactivation. We hypothesised that the eumelanin photophysics is reflected in DHICA monomer be- 
haviour.

Hence, the important hypothesis about eumelanin's excited state deactivation was about the role of the excited state proton transfer, or, in other words, the role of the DHICA monomer as the proposed minimal functional unit, responsible for the ultrafast energy dissipation in eumelanin. We already demonstrated that the DHICA monomer does not experience a decrease of the excited state lifetime upon dimerization. Additionally, we presented the evidence that the DHICA oligomer in an acidic environment, capable of proton transfer, also exhibits the excited-state decay, comparable to that of DHICA monomer. Therefore, the comparison of the DHICA decay with that of eumelanin, which is comprised of aggregates of DHICA and DHI oligomers, will reveal the actual role of DHICA monomer and the role of ESPT from $\mathrm{OH}$ groups to the surrounding solvent.

In this chapter we will study the main energy dissipation hypotheses. We studied the role of the chemical disorder in eumelanin aggregates using a combination of TA and TRPL spectroscopic methods. Additionally, we have compared the results of the ultrafast spectroscopic measurements performed on DHICA monomers with those of eumelanin. We addressed both ESPT and CT states hypotheses in order to reveal the most probable primary excited state deactivation pathway.

The dynamic energy transfer between chromophores of eumelanin was studied with the help of excitation and fluence dependent transient absorption spectroscopy. By tuning the excitation wavelength from NIR to deep UV we could resolve the spectral dynamics. The fluence dependence is an additional tool for extracting information about the mobility of the excitations in eumelanin and calculating the mean distance between the excitations. Polarization-dependent studies of both DHICA and eumelanin were used to address the hypothesis about the formation of CT states.

The proton transfer mechanism in eumelanin films was studied by comparing DHICA and eumelanin's photophysics using the combination of TA and TRPL methods to reveal the full picture of the pigment behaviour. 


\subsubsection{Sample preparation}

All the results presented herein are obtained for synthetic eumelanin purchased from Sigma Aldrich which is produced via the oxidation of tyrosine with hydrogen peroxide. The procedure of preparation of the thin films of synthetic eumelanin was performed as previously described [16]. This approach helps us to avoid some unwanted solvent interactions which may lead to the differences between eumelanin under study and skin eumelanin. The powder was suspended in ammonia water solution ( $2: 1$ by volume, $\mathrm{NH}_{3}(\mathrm{aq})(28 \%)$ in MilliQ water). The resulting suspension was sonicated for 30 minutes to break apart the large aggregates and then stirred for 1 hour. Then the suspension was then spincoated onto a quartz disk to obtain the thin films and evaporate the solvent. The parameters were varied over several spin coatings, but typically $120 \mu \mathrm{L}$ of $30 \mathrm{mg} / \mathrm{mL}$ eumelanin/ammonia solution was pipetted onto the quartz disk, whereupon the disk was accelerated from $665 \mathrm{rpm} / \mathrm{s}$ to $600 \mathrm{rpm}$ for 2 minutes (to disperse the solution), then accelerated from $665 \mathrm{rp}$ $\mathrm{m} / \mathrm{s}$ to $4000 \mathrm{rpm}$ for $30 \mathrm{~s}$ (to evaporate the ammonia solution). Figure 5.1 shows the steady-state absorption spectra of the resulting film.

\subsection{Role of chemical disorder in the energy dissipation of eumelanin}

The chemical disorder model suggests that by exciting the pigment at specific wavelengths, we will excite a specific subset of chromophores absorbing in the selected spectral range. By pumping eumelanin at different excitation wavelengths, we reveal the spectral dynamics of the pigment and discover the role of the energy transfer between chromophores.

For establishing the role of the disorder in eumelanin pigment, we performed five TA measurements in which we excited the thin films of eumelanin at different wavelengths in the range from NIR to UV. Due to the TOPAS performance drop by the time of the start of this project, the earlier data sets for $532 \mathrm{~nm}$ and $712 \mathrm{~nm}$ excitation, obtained by Dr Karen Thorn of Victoria University of Wellington, were 


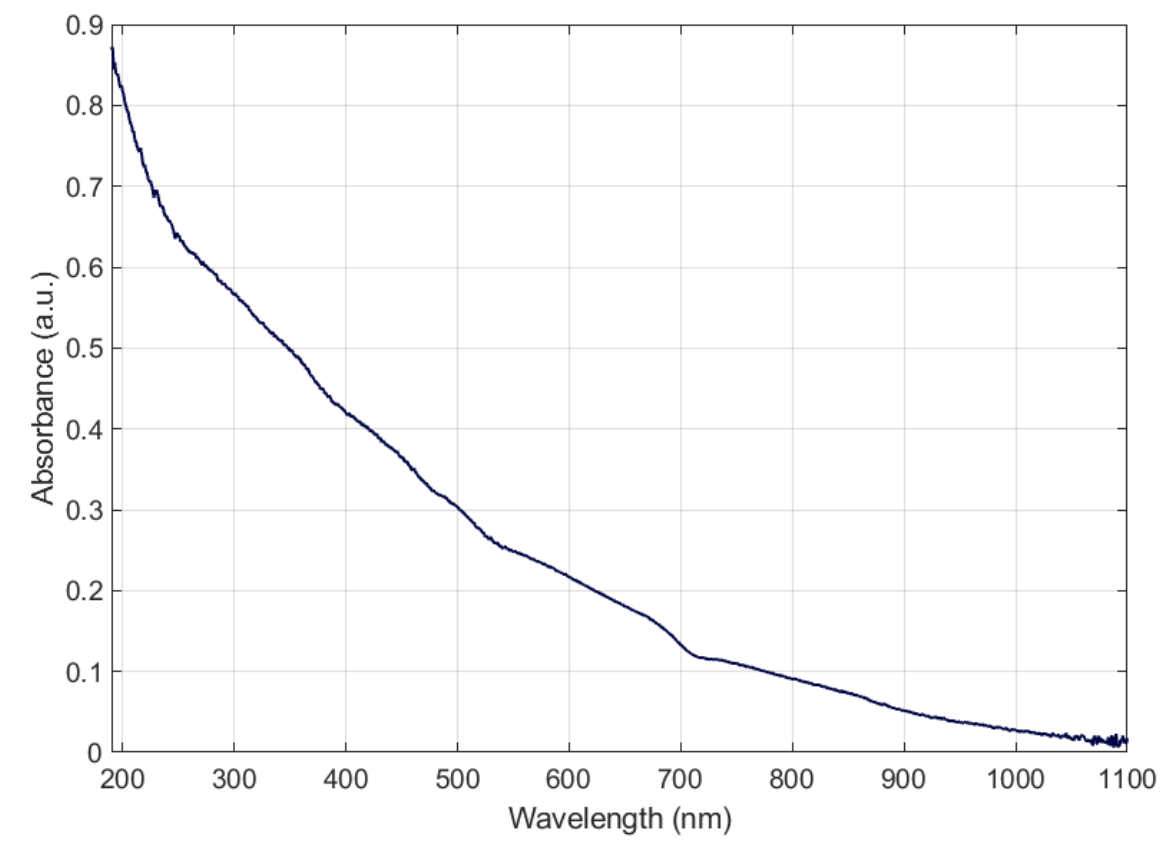

Figure 5.1: Steady-state absorption of eumelanin film.

used. Spectral slices are shown in Figure 5.2.

We observe an appearance of a of a PIA "shoulder" before an actual PIA peak at $\sim 900-950 \mathrm{~nm}$ as the excitation wavelength moves from the NIR towards UV. This might be a sign of an inter-chromophore energy transfer. When the energy is funneled down from the higher energy excited chromophores to the lower energy excited chromophores, the newly excited species will produce their own GSB peak. That way, we would observe a red shift of the GSB peak with time. However, the apparent absence of the red shift does not signify that there is not one, as the spectra of different chromophores would overlap and their GSB and PIA area will cancel each other out creating a long shoulder before an actual peak. The spectral shape for $712 \mathrm{~nm}$ excitation does not have any shoulder following the GSB peak so therefore we would assume that this spectral shape reflects the actual spectrum of the chromophore that is being excited.

To investigate this phenomenon, we performed a Matlab-based simulation that would explore the possible explanations to the changes in the transient absorption 


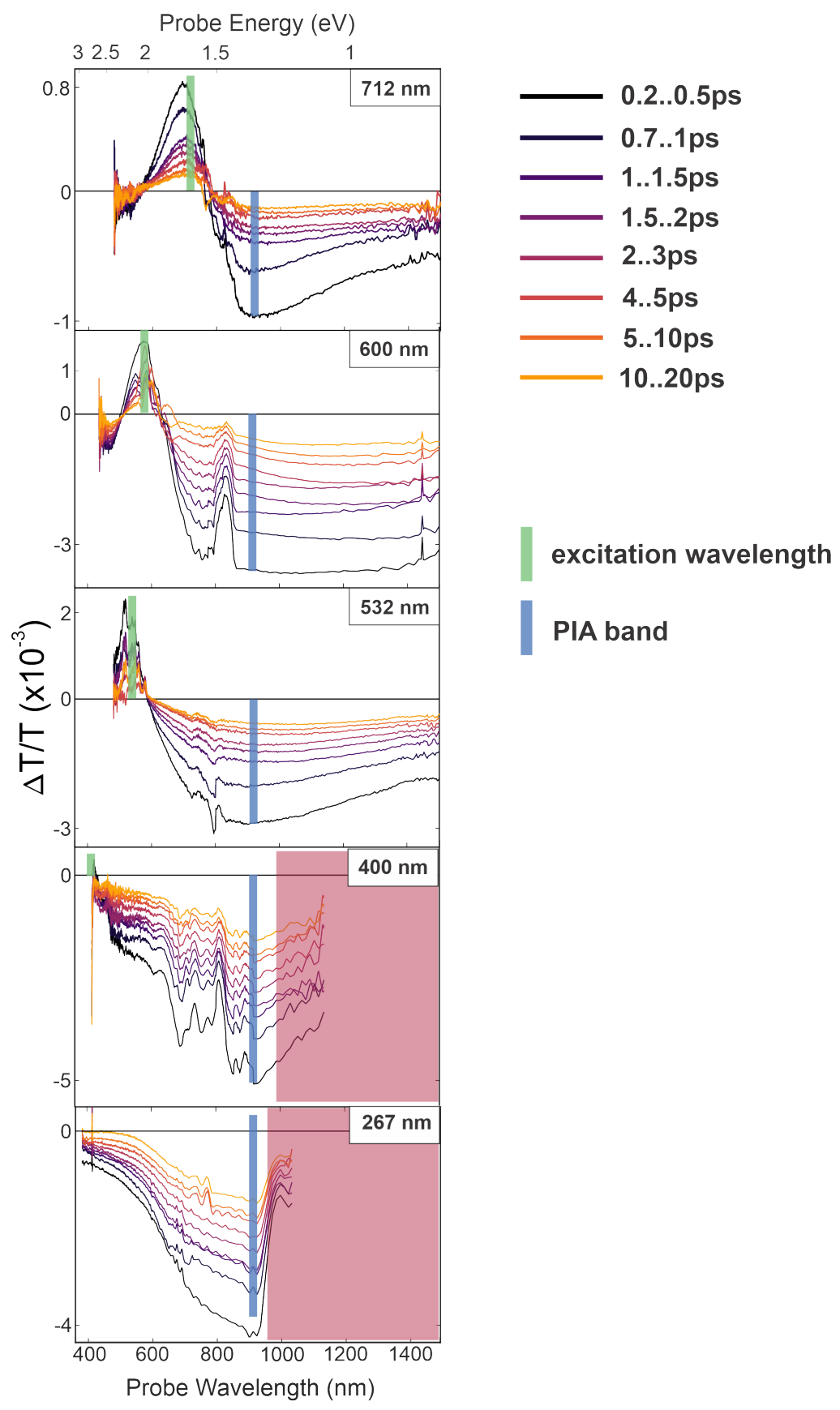

Figure 5.2: Transient absorption spectra of the thin films of synthetic eumelanin, excited at different wavelengths across the spectral range. The shaded area hides the lack of the spectra due to the weak supercontinuum signal. 
spectra as we tune the excitation towards the UV range.

\subsubsection{Matlab-based simulation for energy transfer in multichro- mophore system}

In Chapter 3, Section 3.2.3 we recorded the transient absorption spectra of the key eumelanin building block DHICA (Figure 3.8). It demonstrated the PIA peak at $\sim 490 \mathrm{~nm}$. For this simulation we assumed that the full spectrum would exhibit the GSB peak and the following PIA peak, which was not observed in the experiments due to the white light weak signal in the UV range blow $450 \mathrm{~nm}$. For the simulation's sake, we will then consider that the single chromophore's spectrum is consistent of the GSB peak and the following PIA peak. The easiest way to generate those spectra was to use the sum of two Gaussians - one with positive and one with negative amplitude.

In this section we will study the two different hypotheses, explaining the unusual eumelanin's spectral shape. The first possible explanation is that when one high energy chromophore is excited, the energy will then be funneled down to the lower energy excited chromophores (Figure $5.3(a, b)$ ). Kohl et al. [92] speculated that the excited energy transfer is not the case for eumelanin, however, this process might happen within an IRF region and hence hard to be resolved. The second hypothesis is that we excite not one chromophore but a certain distribution of chromophores, possessing different HOMO-LUMO energy gaps (Figure 5.3 (c)). In this model, the energy of the incoming photon would be split between the chromophores absorbing at the lower energy as depicted in Figure 5.3 (c). Additionally, in comparison with the study of polymerization of DHICA, presented in Chapter 3, Section 3.2.5 we assume that the longer chromophores are absorbing at the longer wavelengths, while still having absorption capacity in the UV range. This assumption was made by studying DHICA dimer and longer aggregates, formed by oxidative polymerization (Chapter 3, Section 3.2.5, Figure 3.20). As proposed in Figure 5.3 (d) different chromophores of eumelanin, while having their absorption peaks across the spectral range, still exhibit a residual absorption peak in the UV range. 


\section{Excitation energy transfer}

(a)

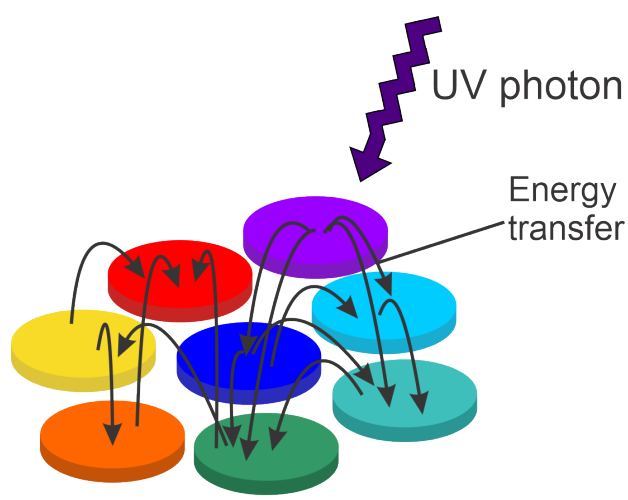

(c)

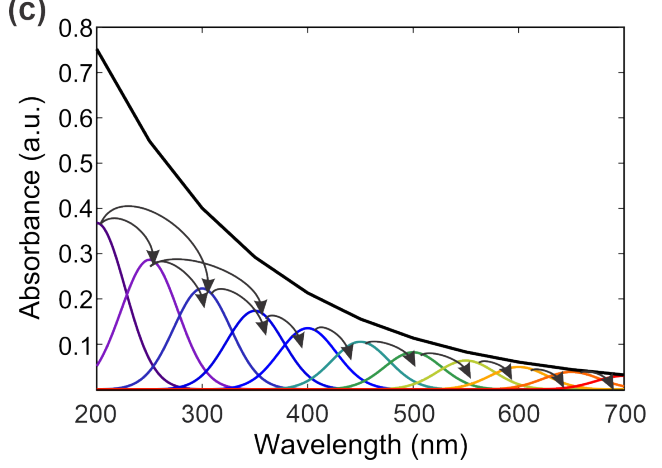

\section{Excitation of the distribution}

(b)

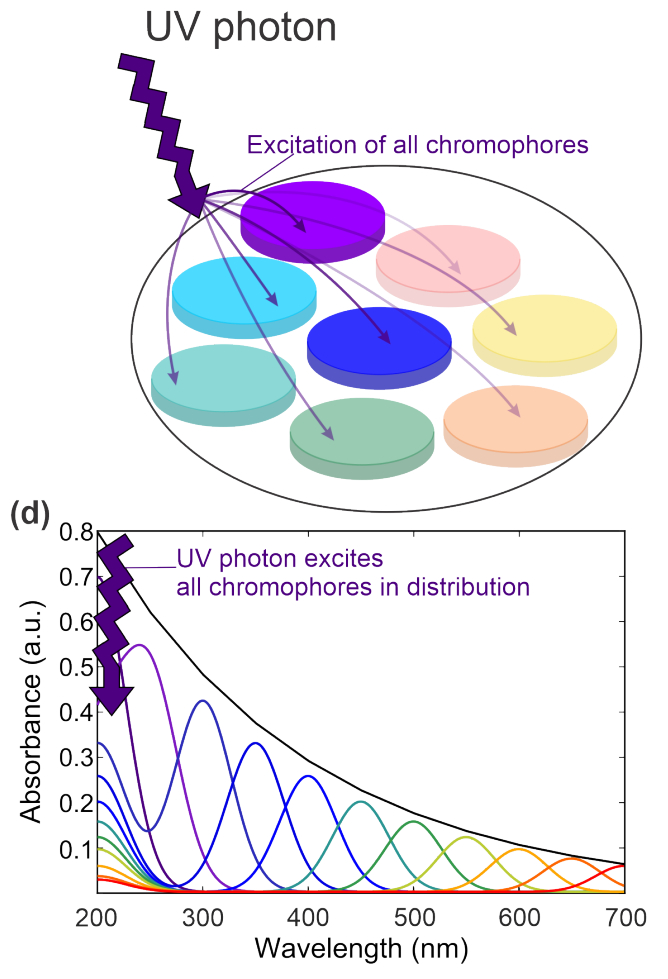

Figure 5.3: Different scenarios of the excitation in the multichromopore system. (a) Excitation energy transfer between the chromophores. The colors are showing the energy decreasing from high (violet) to the low (red) energy; (b) spectral representation of the excitation energy transfer. (c) Excitation of the distribution of chromophores, each having a residual absorption peak in UV and (d) its spectral representation. 


\section{Dynamic energy transfer between chromophores}

In this example we consider the discrete chromophores and the possibility of the energy funneling from a high energy excite chromophore to a lower energy excited chromophore. To study the easiest case of the energy transfer, we simulated different scenarios for rate equations and solved them numerically in Matlab. The resulting code is presented in Appendix A. We started with a simple case for a first order rate equation:

$$
A \rightarrow B \rightarrow C
$$

Then, the change of the concentration of each component with time could be described with the help of a system of differential equations:

$$
\begin{aligned}
& \frac{d A}{d t}=-k_{A B} A, \\
& \frac{d B}{d t}=k_{A B} A-k_{B C} B \\
& \frac{d C}{d t}=k_{B C} B
\end{aligned}
$$

where $k_{i j}$ are the rate constants. Matlab can solve those equations using the function ode, but when this code is to be applied to the real data sets, numerical solutions will be an easier and better choice. So, by using Euler method [115] we transform these equations into

$$
\begin{aligned}
\Delta A & =-k_{A B} A \Delta t, \\
\Delta B & =k_{A B} A \Delta t-k_{B C} B \Delta t, \\
\Delta C & =k_{B C} B \Delta t
\end{aligned}
$$

According to the chemical disorder model, described in Chapter 1, section 1.3.1, chromophores, comprised of DHI and DHICA and their different quinone forms, have different HOMO-LUMO energy gaps and therefore they show the different absorption across the spectra. As was mentioned earlier, we assume that the GSB 
peak was preceding the PIA peak in the transient absorption spectra of the DHICA monomer. Therefore, to simulate the spectra for each chromophore component we used a sum of two Gaussians with positive and negative amplitude. To reproduce the different chromophores, absorbing across the spectral range, we take the simulated TA spectra of DHICA at different points along the spectrum.

Once we modelled the kinetics and spectral traces, we multiply those two matrices to get the TA surface. First, we only consider the simple 3-component system, described by an equation 5.1 By simulating this scenario in Matlab we get the result, presented in Figure 5.4.

For creating the simulation that would be more independent on the input parameters, we tried a simulation that would model the scenario when we set the matrix of rate constants $k_{i j}$ as the only input. There, the program would understand the studied case by estimating the number and the positions of the rate constants in the matrix. If we transform the equation 5.6 to a Matlab-like expression $B(n)=B(n-1)+A(n-1) k_{A B} \Delta t-B(n-1) k_{B C} \Delta t$, where $n$ is the number of time steps, we can try and find the dependence between the components in general terms.

For the matrix of the components, we need to determine the expressions for every row of the rate constant matrix as they each represent one component. We know that each of the component's concentrations can increase or decrease depending on the way the reaction goes. So by using the mathematical induction method we found the expression for the reverse transition between components $m$ and $l$ :

$$
m(n)=m(n-1)-\sum_{i}^{c-1} m(n-1) k_{m i}+\sum_{i}^{c-1} l(n-1) k_{i m}=1
$$

To test the simulation we have chosen five spectral masks, translated along the energy axis. This number of masks have been chosen for simplicity. We simulated the different ways of the excitations hopping between the chromophores -i.e. not just the consecutive energy transfer but also the energy transitions further down to the lower energy excited chromophore. The result is presented in Figure 5.5. It is seen in Figure 5.5 (c) that the resulting sum of the five simulated spectra (thick black line) resembles the spectral shape of eumelanin at short excitation wavelengths. We also 
(a)

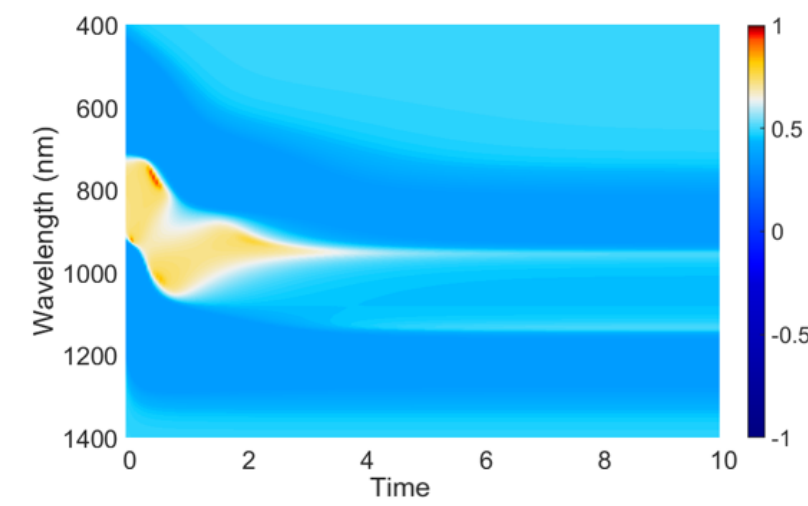

$$
A \stackrel{\mathrm{k}_{\mathrm{AB}}}{\longrightarrow} \mathrm{B} \stackrel{\mathrm{k}_{\mathrm{BC}}}{\longrightarrow} \mathrm{C}
$$

(b)

(c)
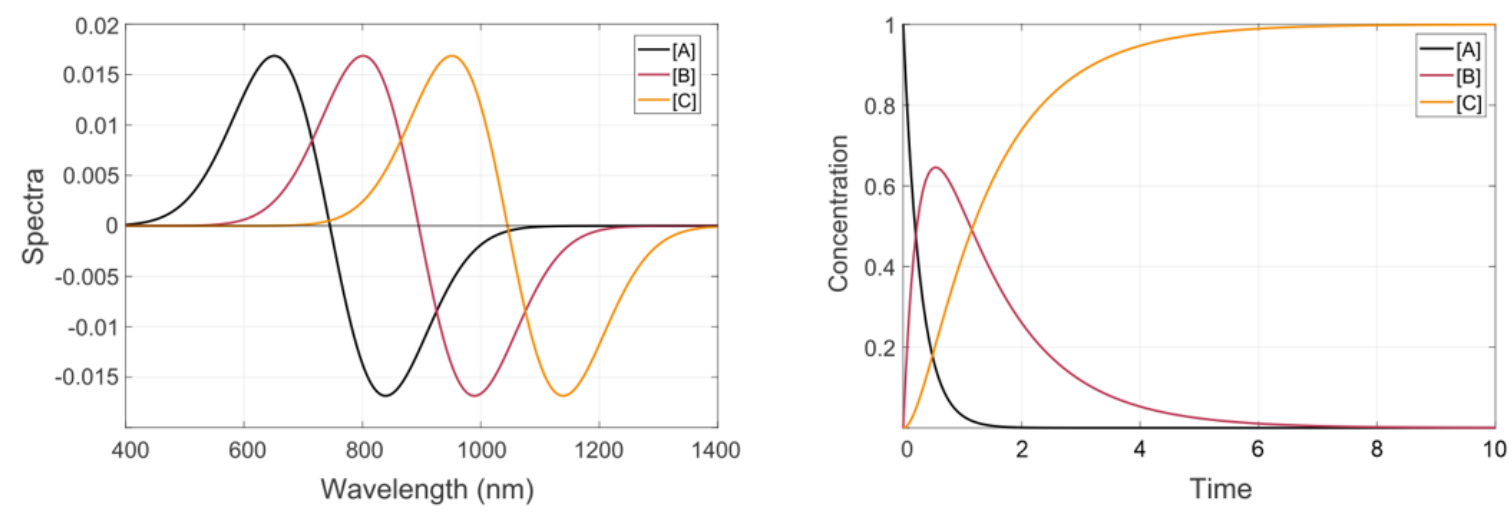

Figure 5.4: Simulated surface (a), consistent of spectra (b) and kinetics (c). 
(a)
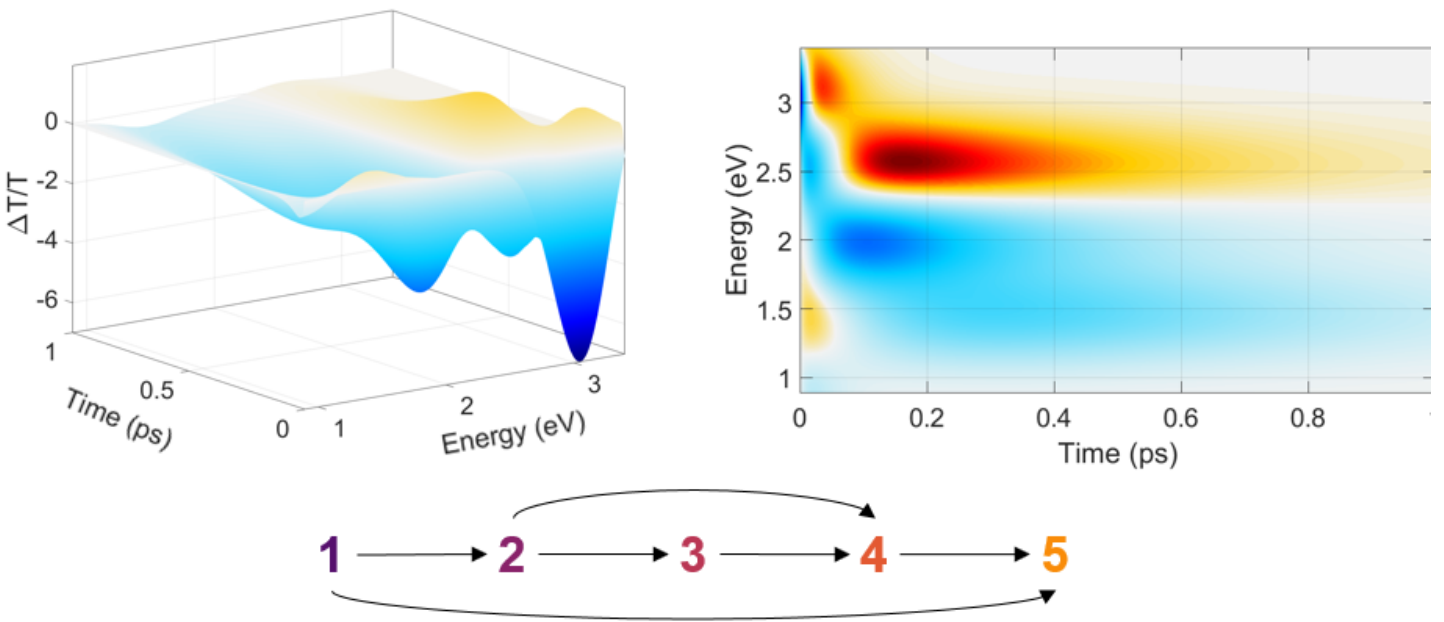

(b)

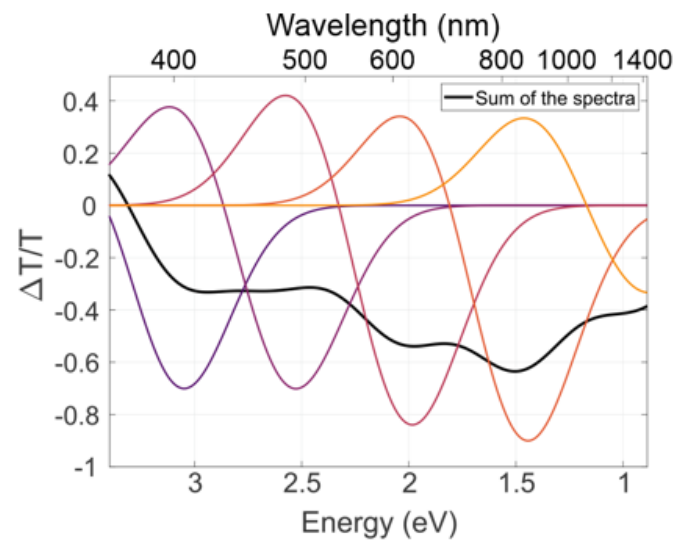

(c)

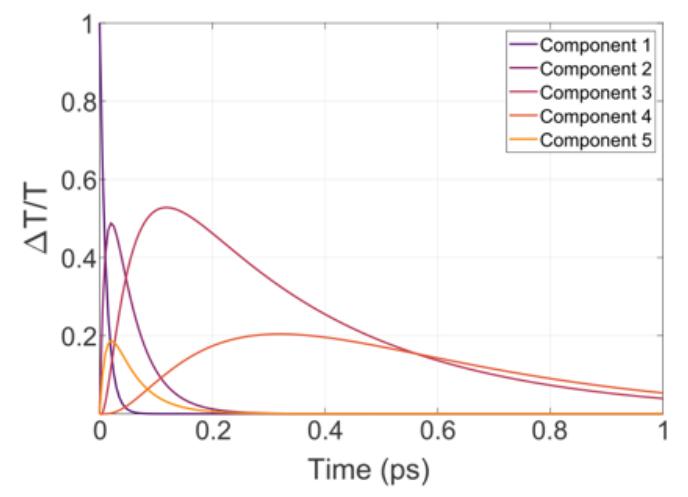

Figure 5.5: The result of the simulated behaviour of the multicomponent system. The presented surface (a) is consistent of the simulated spectra (b) and kinetics (c). 
observe the red shift at early times due to the energy transfer, which is not the case for eumelanin.

The presented simulation only shows the simplified case of the energy transfer between different components and does not take into account the complexity of eumelanin's spectral and kinetics behaviour. However, we note that in the case of the modelled energy transfer we observe the red shift not only for the GSB peak but also in the PIA peak. This result was not discussed in the earlier paper [92], however, this simulation demonstrates that it should not be neglected. The red shift for GSB and PIA peaks was not seen in actual eumelanin spectra (Figure 5.2). The two presented cases demonstrate that no matter how many chromophores we include in the simulation, the result will still include the red shift in both GSB and PIA. The energy transfer, however, could still be happening within $200 \mathrm{fs}$ after the excitation and not be resolved with the used laser setup, as this time range falls into the instrument response function which is the width of two pulses.

\section{Excitation of the chromophores' distribution}

The broad shoulder in the PIA region, which was unusual, compared to the spectra of DHICA monomer, can also be explained using an alternative hypothesis - that we do not excite only one chromophore but a certain distribution. In this example, we will focus on the spectral shape only.

In this example instead of exciting one chromophore that then transfers the energy down to the other chromophores, we assume that we excite a distribution of chromophores. We imply that eumelanin consists of many chromophores that absorb at UV range. This is consistent with its role as photoprotectant. Additionally, DHICA building block's main absorption band also lies in UV part of the spectra, and while creating the longer oligomers it was still capable to absorb UV photons. The redistribution of the chromophores can occur within a laser pulse which cannot be resolved by the currently used laser setup.

To investigate this phenomenon, we have performed a simple simulation to reproduce a long shoulder before the PIA peak. The spectrum for $712 \mathrm{~nm}$ excitation was chosen as a single chromophore spectral shape since it represents the shape that 


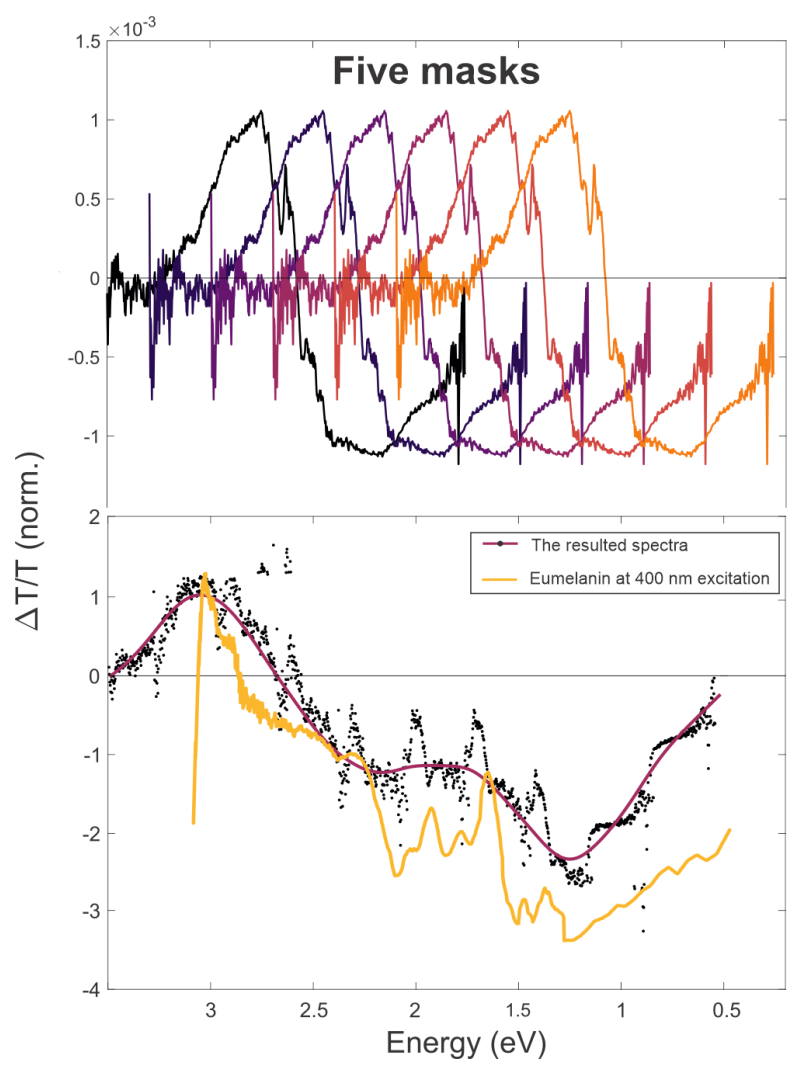

Figure 5.6: Spectral masks translated along the energy axis five times with the $0.2 \mathrm{eV}$ spacing as shown in the top panel. The result shows an agreement with the real data at $400 \mathrm{~nm}$ excitation. The form of the spectral mask was taken from the spectral shape at $712 \mathrm{~nm}$ excitation. 
was chosen to reflect the spectral properties of DHICA monomer - the PIA peak following the GSB peak. We assume that the lower energy excited chromophores can still absorb the incoming UV light within the chromophores distribution. To reproduce the spectral distribution the chosen spectrum was translated several times along the energy axis and then the superposition was taken to observe the new resulting form of the spectra. For better comparison, the result was fitted with a spline function. The observable PIA shape (Figure 5.6) shows a good agreement with the data for shorter wavelengths. The broad shoulder, observed for the modelled spectra (pink curve) appears after the summation of GSB and PIA peaks.

The plateau, that was observed in the real eumelanin's spectral shape, is simply reproduced with the summation of the few spectra of the constituent oligomers, or chromophores. The cancellation of the GSB and PIA peaks of different chromophores resulted in long PIA "shoulder" and PIA peak at $\sim 900 \mathrm{~nm}$ (or $\sim 1.4 \mathrm{eV}$ ). The GSB peak appears only once at the blue end of the spectrum. This is also consistent with the observable GSB shift together with the wavelength of excitation. Therefore, we see GSB peak at the wavelength of excitation because it would be cancelled out only if the higher energy excited chromophores were making a contribution. Since we assume that the spectral shape of the chromophore is the PIA peak following the GSB peak, the GSB peak of the chromophore that is excited by the highest energy has no PIA peak preceding it and hence it is not cancelled out by the summation of the two peaks.

Similar logic applies to explain the PIA peak appearance at the same wavelength for every excitation. To cancel out the PIA peak at $\sim 900 \mathrm{~nm}$, we should excite the chromophores that absorb at the wavelengths $>\sim 900 \mathrm{~nm}$. However, from the absorption spectrum of eumelanin, we know that there are not many chromophores that absorb in that spectral range, and hence it is unlikely to have another GSB peak at that part of the spectra to cancel out the $\sim 900 \mathrm{~nm}$ PIA peak.

The observed results serve as a plausible explanation for the experimentally obtained data. In this hypothesis, the $\sim 900 \mathrm{~nm}$ PIA peak arises from the multiple GSB and PIA peaks of the chromophores' distribution and not from the charge transfer states, as was suggested earlier [92], since this model did not take the CT states into 
(a)

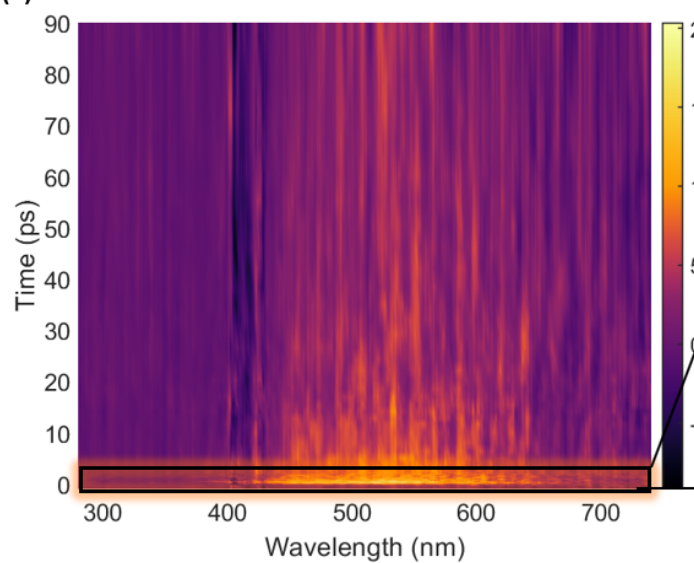

(b)

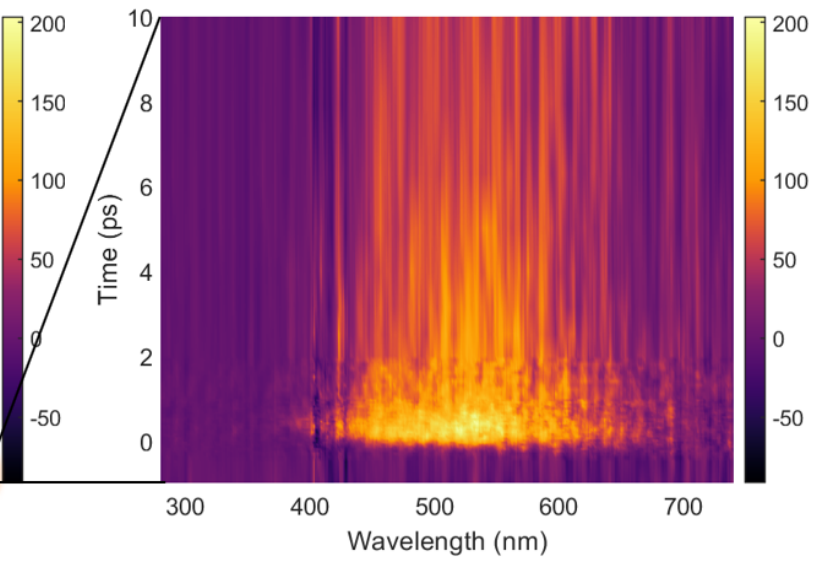

Figure 5.7: Photoluminescence surface for synthetic eumelanin thin film excited at $267 \mathrm{~nm}$ : full surface at 90 ps after excitation (a) and first 10 ps after excitation (b).

consideration. We conclude that this model is the right one to explain the observed eumelanin's behaviour.

\subsubsection{Transient absorption results reveal localized excitations}

Kohl et al. established that the dynamic energy transfer is not the case for eumelanin, based on their TA measurements. They showed the GSB peak that is not redshifting over time, which was indicating immobile excitations. However, as was demonstrated in the previous section, GSB peaks from the lower energy excited chromophores can appear as a result of an energy transfer from a higher energy excited chromophore and overlap with a PIA from another chromophore. Hence, another evidence is needed to rule out the excited energy transfer hypothesis and see the dynamic role of the disorder in excited state deactivation in eumelanin.

Ultrafast TGPL measurements, obtained in collaboration with Isabella Wagner of Victoria University of Wellington demonstrated possible evidence of excitations immobility. We excited the samples of synthetic eumelanin thin film at $267 \mathrm{~nm}$ which is the third harmonics of laser excitation. Figure 5.7 demonstrates the obtained surface. We observe the lack of spectral dynamics (Figure 5.7 (b)), consistent with the picture of localised excitations. 
To investigate whether the excitations are funneled energetically and spatially, or the energy is stored in the chromophore that is being excited, we have performed a series of fluence-dependent TA measurements (Figure 5.8 (a)). It was established that electromagnetic coupling between neighbouring chromophores creates delocalised excitons - electronic excited states whose size and energy depend on the geometrical configuration of the constituent chromophores [156]. The delocalized excited state might give rise to an ultrafast energy relaxation due to excitons interactions in eumelanin.

Exciton-exciton annihilation is established as a decay mechanism in several aggregates of organic molecules [51, 105]. The high exciton densities and the process of exciton diffusion increasing the possibility of excitons to collide within their lifetime. Annihilation occurs once the excitons meet and exchange energy.

Hence, once we excite the sample with the more powerful pump, the created excitons become more mobile and therefore can annihilate at the higher rate. This would result in fluence-dependent kinetics decay, like shown in the comparison for the fused ring electron acceptor IDIC [28], possessing the high exciton diffusion coefficient (Figure 5.8 (b)). However, eumelanin shows no such behaviour - for every fluence used, the decay stays essentially the same, as demonstrated in Figure 5.8 (a), for eumelanin thin film, excited at $400 \mathrm{~nm}$. Similar fluence independence is observed for the rest of the excitation wavelengths as well.

Fluence independence of the kinetics traces shows that the excitations do not interact even at the much higher fluences than IDIC. To get an idea about the distance between the excitations, we first calculate excitation density [70]:

$$
N=\frac{P}{V} \frac{\lambda}{h c}\left(1-10^{-A}\right)
$$

where $P$ is pulse energy, $V$ - volume, illuminated by excitation, $\lambda$ - excitation wavelength, and $A$ is the absorbance at the excitation wavelength. For this example, we chose the TA measurement at $267 \mathrm{~nm}$ excitation, with the highest pump power at $\sim 1 \mathrm{~mW}$. The repetition rate of the pump pulse that went through the chopper is $1.5 \mathrm{kHz}$. From that, we calculate pulse energy $P=$ power $/$ rep.rate $=6.7 \times 10^{-7} \mathrm{~J}$. The volume is calculated from the spot size area and the film thickness. For the 

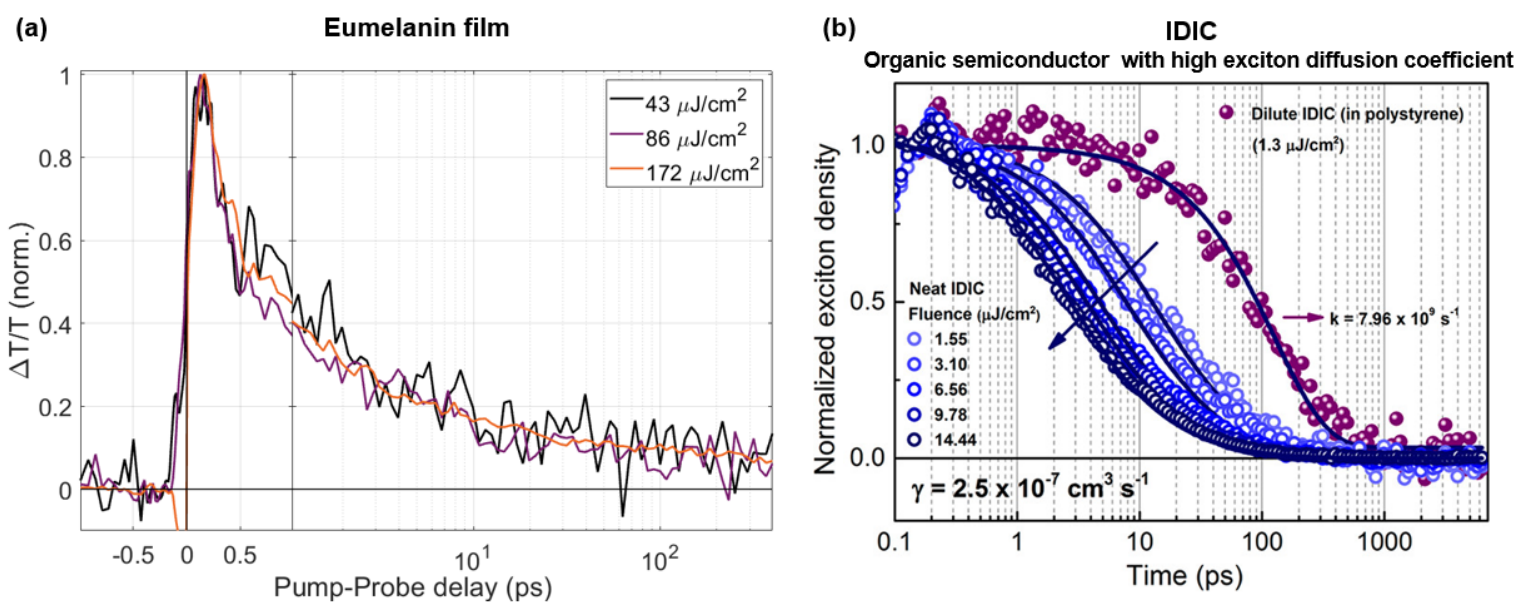

Figure 5.8: Fluence independence for the kinetics traces of eumelanin thin film at $400 \mathrm{~nm}$ excitation (a). Singlet-singlet exciton annihilation in neat IDIC film (b). The figure (b) is reprinted with permission from [28]. Copyright 2019 American Chemical Society.

chosen measurement the spot size of the pump beam at the sample position was $398 \times 271 \mu \mathrm{m}$. The film thickness was calculated from the absorption coefficient of eumelanin film that was taken at the value $10^{7} \mathrm{~m}^{-1}$ for the ultraviolet range [16]. The relationship [3] between absorption coefficient $\alpha$ and the film thickness $d$ is

$$
\alpha=2.303 \frac{A}{d}
$$

Therefore, by extracting $d$, we calculate the film thickness to be $\sim 115 \mathrm{~nm}$. This is in good agreement with the measurements of the inhomogeneous film thickness performed by Dr Karen Thorn of Victoria University of Wellington using Veeco Dektak 150 profilometer. Two different thicknesses were obtained for the same film at different positions: $70 \mathrm{~nm}$ at the first position and $160 \mathrm{~nm}$ at the second position. Using the estimated film thickness value we calculate excitation density to be approximately $7.2 \times 10^{19} \mathrm{~cm}^{-3}$ - it is a rather high value for the given high fluence and film thickness. For comparison, excitation density for conjugated polymers like Y6, in which the excitations are mobile and hence the materials would demonstrate the fluence dependence, would be $0.5-60 \times 10^{14} \mathrm{~cm}^{-3}$ [140]. Knowing the excitation density, we can estimate the mean distance between the excitations: 


$$
r=\sqrt[3]{\frac{1}{N}}
$$

The estimated distance between the excitations was found to be $2.4 \mathrm{~nm}$. This value is rather small - in $\pi-\pi$ conjugated systems, where the excitation density is lower and the distance between the excitations is larger, the excitations are still mobile, showing a facile exciton diffusion [28]. However, in eumelanin even at a short distance, when the excitation density is high, the excitations are not mobile enough to interact within such a small radius. It should be noted that this value is not a limit for the mean distance between the excitations. The excitations could be confined to a smaller radius, which can be calculated if a higher excitation fluence was used. In this example, we estimate that the excitations are confined to at least $2.4 \mathrm{~nm}$. For a better estimation, we compare that radius to the length of DHICA dimer, which was measured to be $\sim 1 \mathrm{~nm}$ in Maestro Molecular Modeling Interface. Knowing that eumelanin is comprised of the chromophores of different lengths that are greater than that of DHICA dimer, we can say that the excitations in eumelanin are rather immobile.

That leads to the conclusion that the excitations in eumelanin are localised and dissipated in the chromophore that is being excited. In this case, we propose that the elongation of the PIA part of the spectra as the excitation energy moves toward $\mathrm{UV}$ region is the result of exciting distribution of the chromophores.

\subsubsection{Charge transfer states recombination}

Polarization anisotropy $[79,96]$ is a powerful tool providing structural information about the material. When a material with randomly oriented chromophores interacts with the light of specific polarization, the amount of chromophores being excited depends on the direction of their transition dipole moments with respect to the polarization of incident light. Therefore, if the chromophores' transition moments are aligned with the direction of polarization of the incident light, the probability of excitation of these chromophores will be higher. If the excitations migrate there will be changes in the orientations of their dipoles and thus the changes in the material's 
anisotropy.

The anisotropy $r$ can be identified [96] as

$$
r=\frac{I_{z}-I_{y}}{I_{x}+I_{y}+I_{z}}=\frac{I_{z}-I_{y}}{I_{\text {total }}}
$$

When excitations are polarized along $z$-axis, the emission is symmetric around the $z$-axis. Hence we can replace $I_{x}=I_{y}=I_{\perp}$ and $I_{z}=I_{\|}$[96]. The anisotropy can then be calculated as

$$
r=\frac{I_{\|}-I_{\perp}}{I_{\|}+2 I_{\perp}},
$$

where $I_{\|}$and $I_{\perp}$ are parallel and perpendicular intensities. When the absorption and emission dipoles are colinear, there are no processes resulting in depolarization. In that case, initial anisotropy is at its maximum value of 0.4 . Under these conditions, the excited-state population is distributed along $z$-axis and $I_{\|}=3 I_{\perp}$ [96].

Transient hole burning measurements by Kohl et al. [92] were used to exclude the possibility of excited energy transfer between chromophores. Our excitation wavelength and fluence dependent TA measurements confirmed that hypothesis, showing that the excitations are confined to the $<2.4 \mathrm{~nm}$ radius. As an alternative excitation deactivation, it was proposed that eumelanin is capable of creating immobile CT states. This hypothesis was supported by the polarization anisotropy measurements. The GSB band was keeping its polarization unaffected, which indicated that the excitations did not move. On the other hand, the PIA band experienced fast depolarization (Figure 5.9 (a)). This was claimed to be a result of the CT states formation between neighbouring chromophores with different dipoles orientations. When the charge moves, the dipole reorients which results in the change in anisotropy. However, CT transitions have low oscillator strength [103], and therefore their PIA signature would be fairly weak. The observed PIA of eumelanin for both our and previously published data is rather strong and prevalent across the spectral range. Therefore, the formation of the CT complex does not sound like a plausible explanation of the photophysical processes in eumelanin.

There are two other possible explanations for the observed anisotropy. In Chap- 
(a)

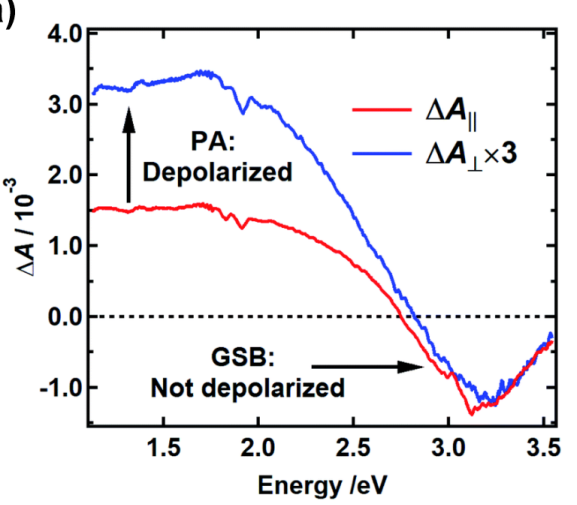

DHICA monomer

(b)

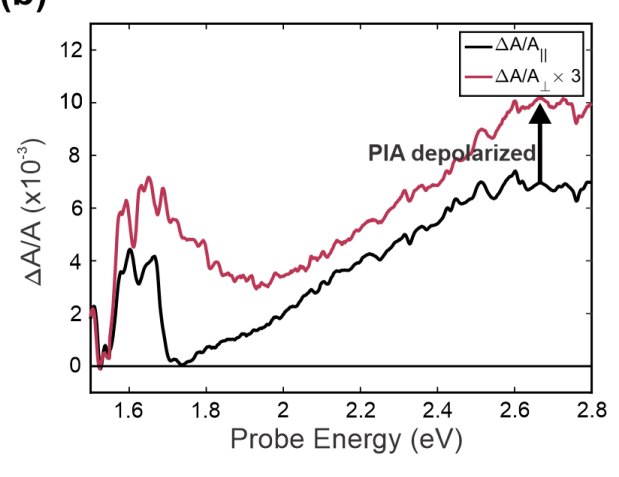

\section{Eumelanin film}

(c)
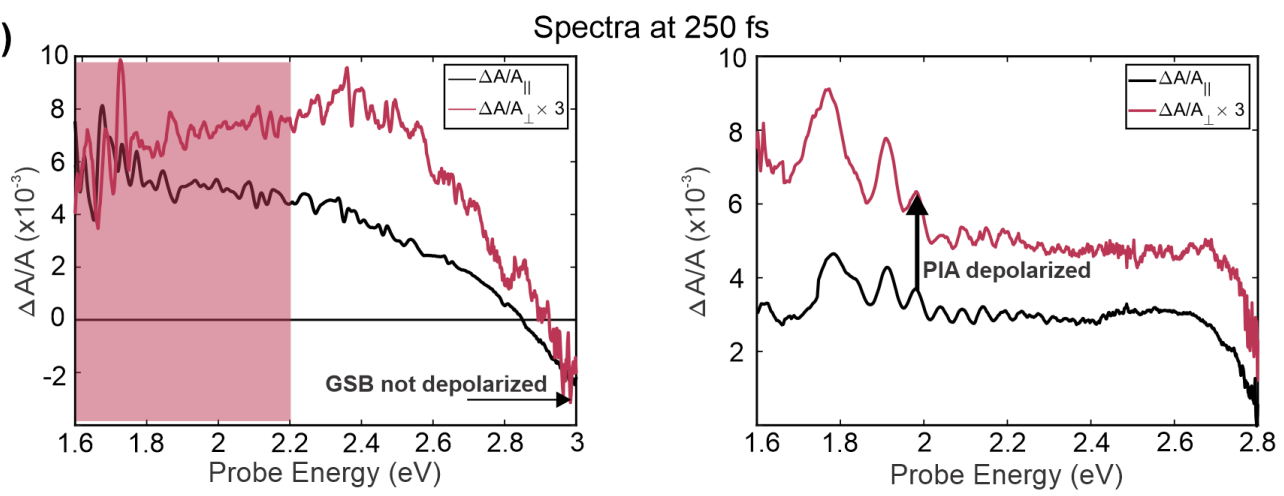

Figure 5.9: Previously published polarization-dependent spectra of the synthetic eumelanin. Reproduced from [92] with permission from the Royal Society of Chemistry (a). Compared with the results of polarization-dependent TA spectra at $250 \mathrm{fs}$ for DHICA monomer (b) and eumelanin film (c). 

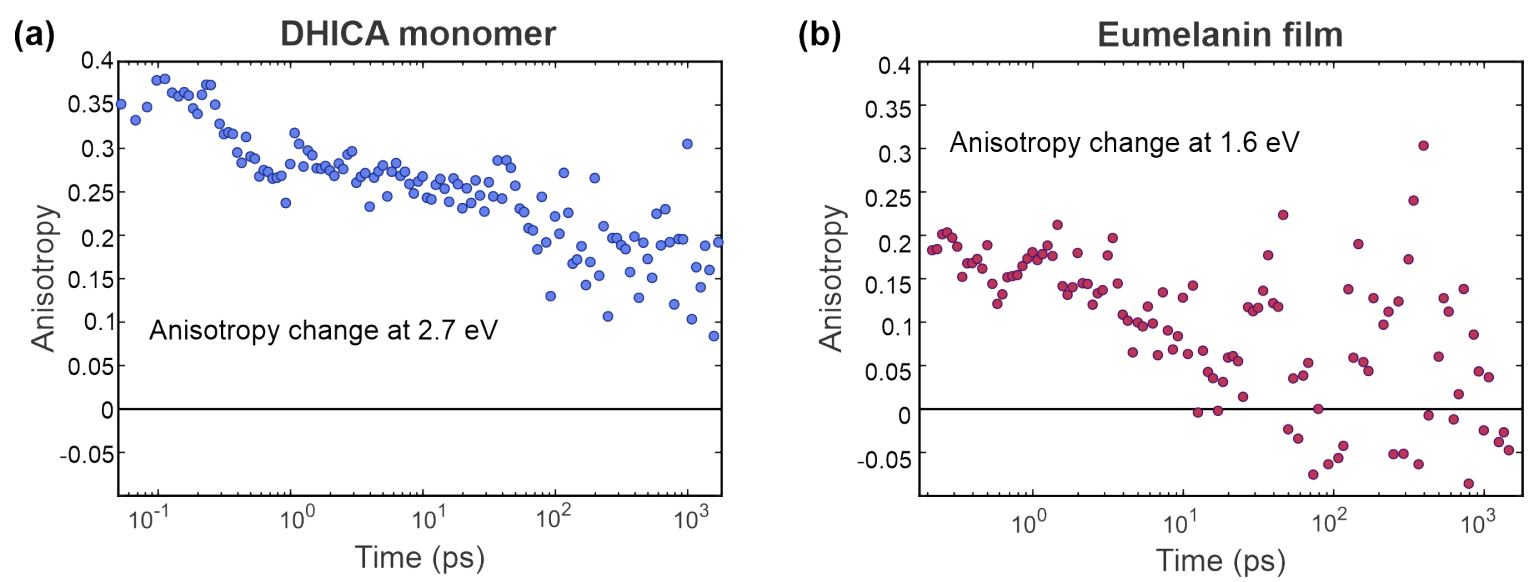

Figure 5.10: The change in anisotropy for PIA, calculated for DHICA monomer (a) and eumelanin (b).

ters 3 and 4 we demonstrated the importance of DHICA for eumelanin. One of the possible reasons for the fast depolarization of the PIA band might be an intrinsic depolarization of the DHICA dipole. This will be a contradiction to the hypothesis about CT states formation because DHICA monomer is unable to create an intramolecular CT state. Another explanation originates from the excitationdependent TA measurements and the spectral behaviour modelling that was described in section 5.2.1. This hypothesis suggests that we excite an ensemble of chromophores in distribution and the resulting spectral shape observed experimentally is coming from the overlapping contributions of different chromophores along the spectrum. Those chromophores at lower energy responsible for the net PIA can possess different dipoles orientation from the higher energy bleached chromophores and that would lead to the observed depolarization without assuming a CT formation.

The polarization-dependent TA spectroscopy of eumelanin film demonstrated the same PIA depolarization, as was found before [92]. Figure 5.9 demonstrates two spectra for the parallel and perpendicular polarizations, scaled by a factor of 3 at $250 \mathrm{fs}$ for DHICA monomer and eumelanin film. The change in anisotropy for the PIA band, calculated as per equation 5.13 is presented in Figure 5.10. For DHICA monomer we observe that the anisotropy starts at a little below 0.4 which 

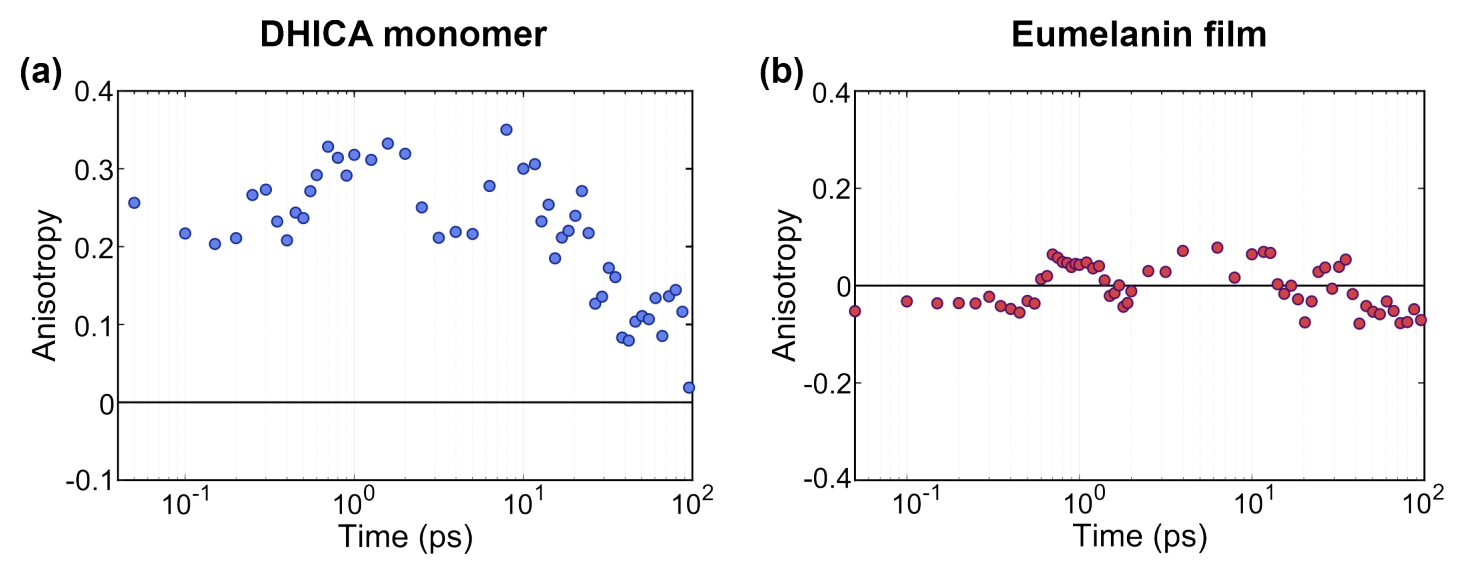

Figure 5.11: Photoluminescence polarization anisotropy of DHICA monomer (a) and synthetic eumelanin film (b).

indicates that the dipole moment of the DHICA monomer is rotated and hence it demonstrates an intrinsic depolarization. With time DHICA monomer underwent ultrafast PIA depolarization (Figure 5.10 (a)). The observed anisotropy decay within a few picoseconds is consistent with ESPT in the DHICA monomer. Hence, since the depolarization is unlikely to be a result of the CT states formation in eumelanin, the other explanation of the observed PIA depolarization is needed.

The anisotropy decay for eumelanin starts at 0.2 and decays within $10 \mathrm{ps}$, as presented in Figure 5.10 (b). The rest of the plot is dominated by noise, as per equation 5.13 for calculating the anisotropy, we divide the sum of the parallel and perpendicular contributions to the full signal, which becomes low at the late time points. We assume that the depolarization from 0.4 to 0.2 happened in the ultrafast timescale within IRF. We propose that this behaviour is likely associated with the rapid redistribution of excitations within a laser pulse and their localization in one chromophore.

The hypothesis about exciting the distribution of the chromophores, described in section 5.2.1 can explain the fact that PIA band undergoes fast depolarization while GSB band does not. We assume that the pump beam excites an ensemble of chromophores that contribute to the elongation of the PIA band. That way the observed GSB and PIA peaks originate from different chromophores' excitation in an ensemble, while the middle plateau is a result of cancelling out the GSB and PIA 
peaks from the other chromophores in the distribution. In the case of GSB band, the only chromophore that contributes is the initially excited one. The PIA band, however, results from the redistribution of the chromophores that happens within the first $200 \mathrm{fs}$ after excitation. This fast distribution will result in the transition dipole moment rotation and therefore the initial anisotropy will show depolarization.

In the case of TGPL (Figure 5.11), DHICA anisotropy starts at the lower position of 0.2 , which happens because of the rotation of emission dipole with respect to absorption dipole. Eumelanin film, however, demonstrated the initial loss of polarization anisotropy, that remained at the zero value during the whole timescale of the experiment. We associate this lack of anisotropy with fast delocalization of excitons on a femtosecond timescale, within IRF beyond the experimental resolution. This is consistent with the already observed TA data. We assume that the photoluminescence occurs from the chromophores that redistributed within the pulse.

The demonstrated polarization anisotropy of DHICA has ruled out the hypothesis of the charge transfer states formation. As DHICA is in its monomer state, it is unable to form the CT states, as the CT state is forming either intramolecularly, or as a result of the charge transfer between eumelanin's chromophores [92]. Therefore, we can confirm that the depolarization of the PIA band is an intrinsic property of the DHICA key building block, and not the signature of the forming CT state. Lack of depolarization of GSB peak and fast depolarization of PIA band originates in the ultrafast redistribution of the ensemble of chromophores. This redistribution occurs within the first 200 fs after the excitation and contributes to the prolonged PIA band.

\subsection{Proton transfer mechanism in eumelanin}

We have concluded that the excitations in eumelanin are localized and immobile. Therefore, it was proposed that the small molecules, such as DHICA monomers, are responsible for the excited state deactivation.

Excited state proton transfer was confirmed to be the main dissipation mechanism in key building block DHICA. In Chapter 3 we demonstrated that in physiological conditions of the human body the DHICA monomers undergo rapid excited 


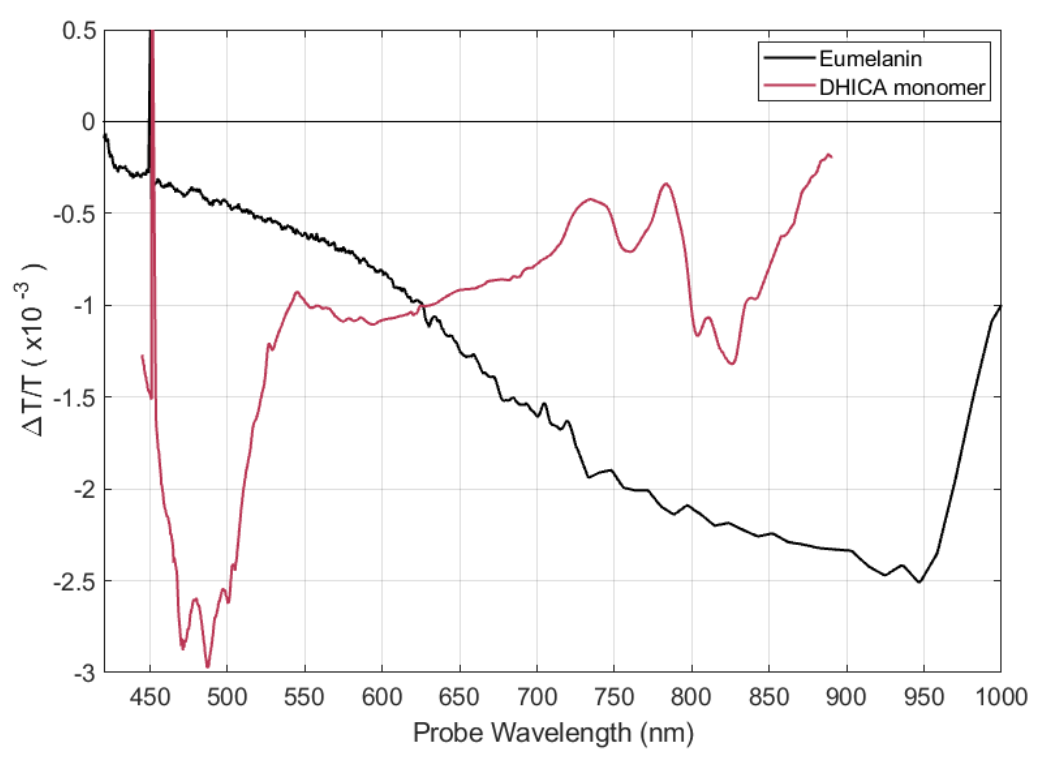

Figure 5.12: Comparison of the TA spectra of eumelanin and DHICA monomer in degassed water.

state deactivation via ESPT from OH groups to the solvent. In the neutral conditions when DHICA is in its monoanion form, the rapid relaxation occurs through the potential energy surface crossing along the reaction coordinate.

Furthermore, in Chapter 4 we have shown that the dimerization of DHICA does not affect the excited state deactivation. In fact, the two decays are identical, which leads to a question about the role of DHICA monomer in the eumelanin pigment's dissipation of absorbed energy. For that, we compared the spectral and kinetics features of DHICA and eumelanin in order to reveal the similarity or difference in the behaviour of these two systems.

First of all, by comparing the transient absorption spectra of eumelanin and DHICA monomer in degassed water (Figure 5.12), we see a major difference. While DHICA only exhibits one PIA peak at $\sim 490 \mathrm{~nm}$, eumelanin's spectral shape is more broad and reaches the NIR region. In Section 5.2 of this chapter we tried to explain such behaviour. We propose that by exciting eumelanin in the UV part of the spectra, we actually excite a distribution of chromophores. Those chromophores, comprised of DHI and DHICA and their various quinone forms, presumably have 

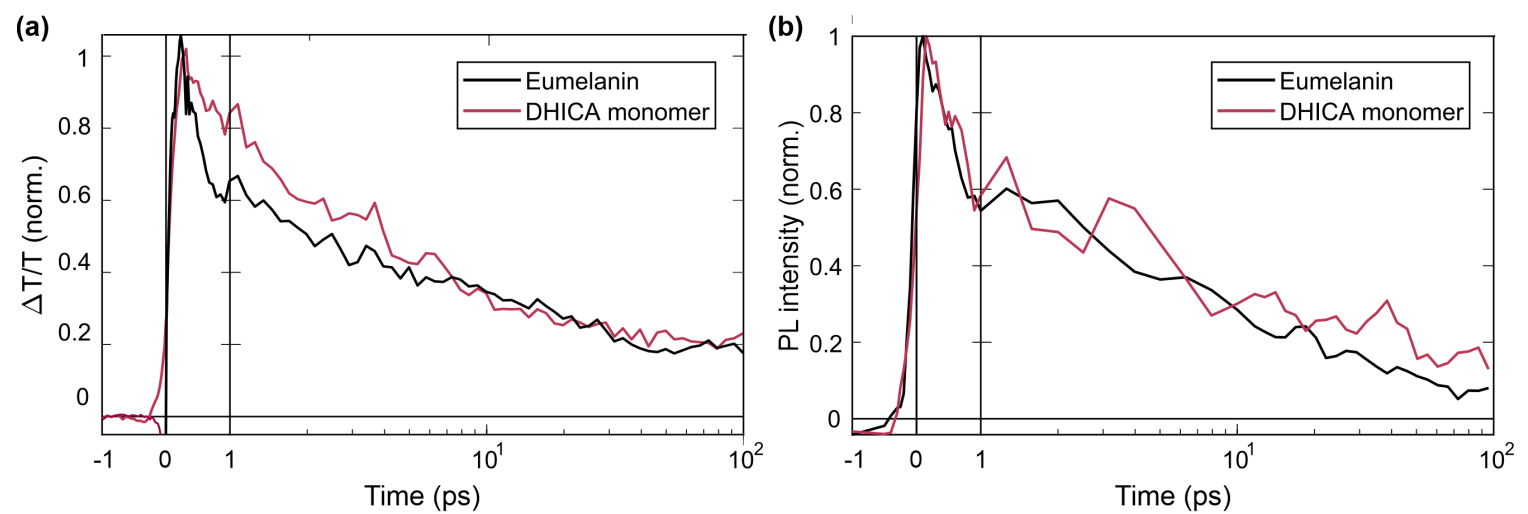

Figure 5.13: TA (a) and TRPL (b) excited state kinetics of DHICA monomer and eumelanin film.

different HOMO-LUMO gaps. This is consistent with the chemical disorder model, discussed in Chapter 1, Section 1.3.1. Therefore, these different chromophores' overlapping TA spectral features result in the long shoulder before an actual peak in NIR.

We are now applying the probes of the proton transfer dynamic to address the question of the role of the ESPT process and, therefore, the role of DHICA monomer in eumelanin excited state deactivation. Having performed both TA and TRPL measurements and analysis of the two systems, we are able to compare their excited state dynamics. The results are presented in Figure 5.13. We observe a very close similarity of both TA and TRPL decay kinetics of eumelanin and DHICA. The weighted average lifetime was found to be $4.27 \mathrm{ps}$, which is in very good agreement with the previously found lifetime of DHICA monomer of $4.25 \mathrm{ps}$, that was assigned to the ESPT from $\mathrm{OH}$ groups to water.

For all our experiments we use eumelanin in thin film state, and not in suspension, like in the case of DHICA. When the suspension is prepared, we need to use a high $\mathrm{pH}$ to dissolve eumelanin, leaving it mostly deprotonated and, therefore, not capable for proton transfer. It is related to that in high $\mathrm{pH}$ we deprotonate any acid/base moieties [16]. The basic ammonia $\mathrm{NH}_{3}$ scavenges the protons from eumelanin and forms an ion $\mathrm{NH}_{4}^{+}$, that deprotonates eumelanin. When the prepared suspension is spincoated, the ammonia solution is evaporated, and $\mathrm{NH}_{4}^{+}$ions send the protons back to eumelanin, leaving it in protonated form. The prepared film 


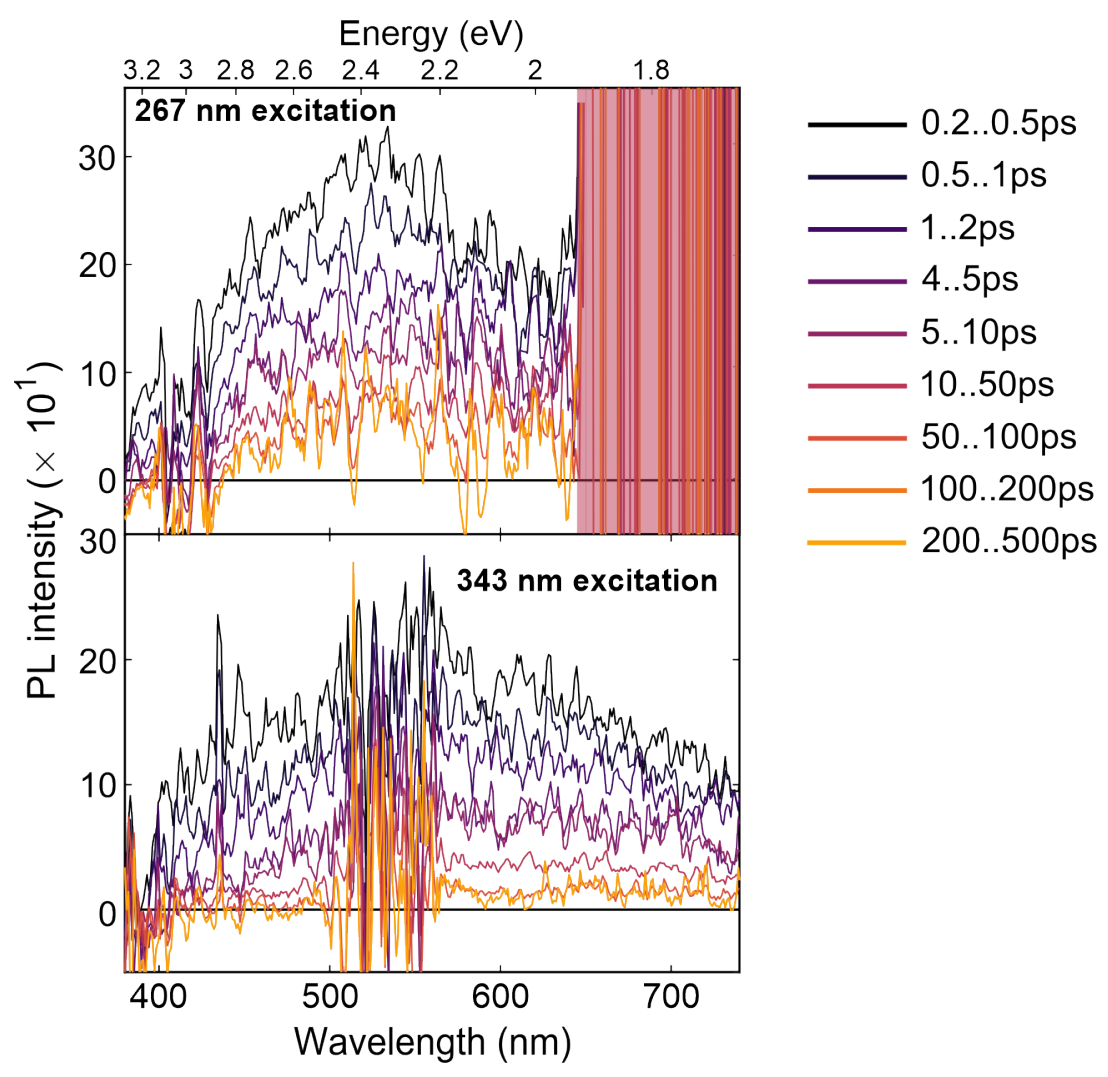

Figure 5.14: TRPL spectra of eumelanin film, excited at $267 \mathrm{~nm}$ (top panel) and $343 \mathrm{~nm}$ (bottom panel).

likely stays wet, i.e. water containing, which allows the protons from $\mathrm{OH}$ groups to be transferred to the surrounding water.

Therefore, we can confirm that eumelanin, which is capable of proton transfer in conducted measurements, exhibits the same ultrafast decay as its key building block DHICA. The close similarity of the two decays indicates the possibility of ESPT mechanism in eumelanin and accountability of DHICA monomers for the pigment's ultrafast decay.

Ultrafast TGPL spectroscopic data, obtained in collaboration with Isabella Wagner of Victoria University of Wellington, showed a wavelength-dependent broadband spectral response, presented in Figure 5.14. The fluorescence peak in both cases lies in visible region, while the samples were excited with two different UV wavelengths. That indicates a large energy loss from excitation ( $4.95 \mathrm{eV}$ and $3.61 \mathrm{eV})$ 
to emission (2.38 eV and $2.29 \mathrm{eV}$, respectively). The initial fluorescence peaks beyond $500 \mathrm{~nm}(2.48 \mathrm{eV})$ suggest that a substantial amount of the absorbed energy is lost within $200 \mathrm{fs}$ (which is the temporal resolution of the used setup) after UV excitation. Such a large Stokes shift is consistent with coupling to a large amplitude motion like ultrafast ESPT [35].

In Chapter 3, Section 3.2.2 we calculated the driving force of ESPT for DHICA monomer in neutral conditions. To do so, it is essential to ascertain the excited state energy difference between the conjugate acids and conjugate bases. However, while in DHICA we could obtain those forms by putting the molecule in different environments, it is unclear how to retrieve this information in eumelanin. We demonstrated that the excited state of eumelanin is not much lower in energy than DHICA, even though it contains longer chromophores. In fact, the difference between these two energies is $\sim 0.6 \mathrm{eV}$. This means that even being excited in the UV range, eumelanin loses a lot of energy in internal conversion, and leads to the assumption that, like protonated state, the deprotonated state is also lower in energy comparing to that of DHICA. Hence, as we observe the PL peak to be lower in energy, one would expect the excited state driving force to get lower for longer chromophores, present in eumelanin.

Additional evidence of the importance of the DHICA monomer in eumelanin photophysics is shown by the comparison of vibrational peaks positions for DHICA monomer and eumelanin [170], collected by Dr Karen Thorn of Victoria University of Wellington, using femtosecond stimulated Raman spectroscopy [95, 106, 107]. Both samples were excited at $267 \mathrm{~nm}$. Figure 5.15 (a) shows the individual spectral slices for eumelanin in thin film state and DHICA monomer in degassed water. We observe a shift in frequency of the initial excited state vibrational peak frequencies in eumelanin to ones similar to DHICA (by comparing the peaks black curves for both eumelanin and DHICA). From Figure 5.15 (b) it is clear that while at the beginning of the measurement the peak positions differ from one another, at later times the frequency peaks for each sample start to more closely match each other. This is believed to indicate the presence of DHICA monomer vibrational signature in eumelanin, meaning that once eumelanin gets excited the energy is funneled through 

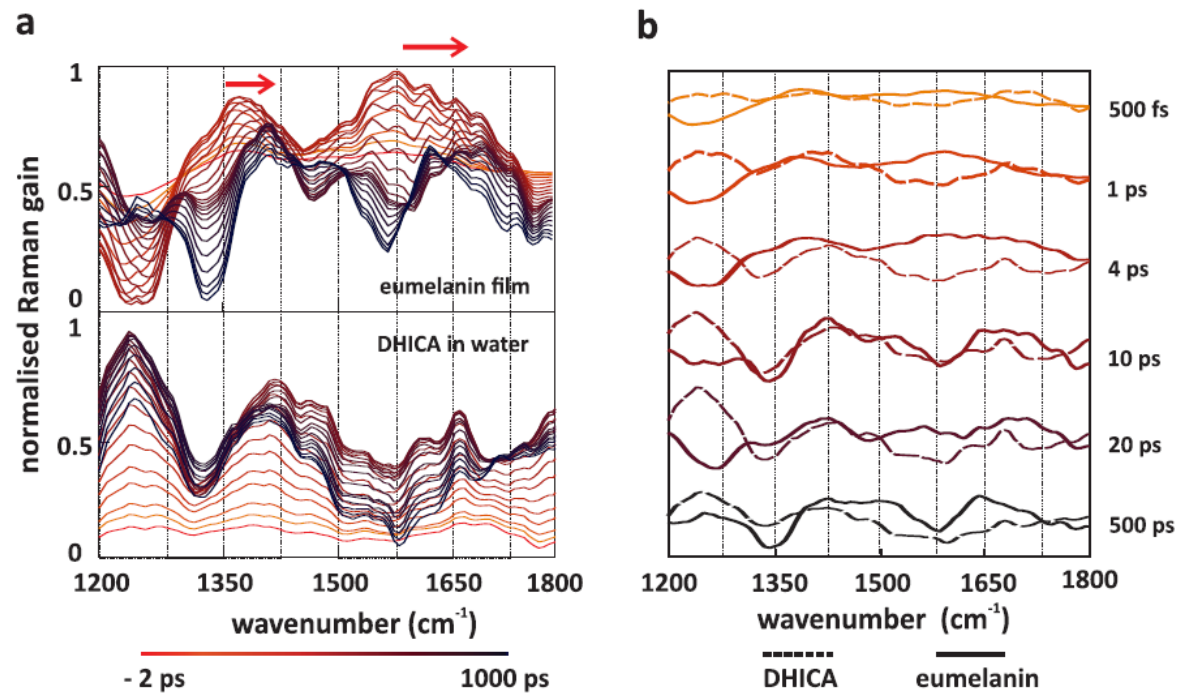

Figure 5.15: Vibrational spectra for eumelanin and DHICA monomer (a); Individual spectral slices (b). The data is collected and presented by Dr. Karen Thorn of Victoria University of Wellington and reprinted with permission from [170] with the provisions of the Copyright Act 1994 (New Zealand).

vibrational modes of DHICA monomer. One of the key vibrational modes shifts around $1600 \mathrm{~cm}^{-1}$ is represented in both eumelanin and DHICA. It represents the vibrations of the molecule due to the deformation of the pyrrole and benzene rings in the absence of wagging of the hydrogen in the phenol group upon deprotonation. This confirms the importance of the DHICA in its monomer state for excited state dissipation in eumelanin.

Taking these findings together, we can confirm that eumelanin dissipated the absorbed energy through the excited state proton transfer from $\mathrm{OH}$ groups and that the DHICA monomer, in which the proton transfer was proved to be the main dissipation mechanism, is reflecting the pigment's photophysics.

\subsection{Conclusion}

In this chapter, we reviewed the different hypotheses for the excited state deactivation in eumelanin. We demonstrated that the disorder of the system does not 
play a dynamic role in the energy dissipation of the pigment. This was supported by the absence of the spectral dynamics of the pigment, as well as the fluence independence of the kinetics decays. We confirm that the energy dissipation is localized in the chromophore that is being excited, meaning that all the excitations are immobile. Having calculated the mean distance between the excitations at the highest fluence, we confirm that they are confined to $<2.4 \mathrm{~nm}$. With the help of polarization-dependent TGPL spectroscopy, we demonstrated that the ultrafast delocalization of excitations happens within a lifetime of $<200 \mathrm{fs}$, which is within experimental instrument response function.

Polarization-dependent transient absorption spectroscopy demonstrated that the hypothesis about the formation of the immobile charge transfer states in eumelanin aggregates is not precise for not including DHICA role into consideration. We established that DHICA monomer, unable to form charge transfer states, are exhibiting the polarization anisotropy of PIA band like eumelanin, and therefore, we conclude that the depolarization of the PIA band in eumelanin, observed earlier [92] is actually the intrinsic property of DHICA key building block. We explain the observed depolarization of the PIA band and the lack of depolarization of the GSB band with the hypothesis of the excitation of the ensemble of chromophores, resulting in GSB and PIA signatures from different chromophores. The PIA band contains multiple GSB and PIA peaks from the ensemble of the excited chromophores that are cancelled out in the middle, creating a plateau, and the observed peak is the result of the redistribution in the vicinity within ultrafast timescale, which results in rapid depolarization.

The comparison of the ultrafast dynamics of eumelanin and DHICA monomer revealed a very close resemblance between the two. The excited state lifetime of eumelanin was calculated to be $4.27 \mathrm{ps}$, which is essentially the same as the DHICA monomer lifetime of $4.25 \mathrm{ps}$. Hence, we detect a close similarity of the photophysics of those two systems. Additionally, the convergence of femtosecond Raman spectra for DHICA and eumelanin confirms the suggestion that DHICA monomers are the ones responsible for the eumelanin's photophysics. Furthermore, the large Stokes shift, observed in eumelanin's photoluminescence peak and the broadness of the 
spectra are also consistent with the proton transfer mechanism. Therefore, we propose that the DHICA monomer is the minimal functional unit, responsible for the effective ultrafast energy dissipation in eumelanin. Knowing that DHICA has the confirmed excited state proton transfer from the $\mathrm{OH}$ group as the main excited-state decay mechanism, we propose the ESPT from phenol groups to the solvent to be the main energy dissipation mechanism in eumelanin pigment. 
- Chapter 6

\section{Conclusions and future outlook}

\section{Conclusions}

In this thesis, we have studied the possible UV energy dissipation mechanisms in eumelanin pigment. To this day, the studies of eumelanin were not able to reveal the primary mechanism of photodissipation, speculating different hypotheses. The complex nature of the pigment, its high degree of disorder and an ultrafast excited state deactivation significantly complicate the studies.

This thesis set out to answer the following questions:

- What are the roles of the key building blocks in energy dissipation?

- How does the oligomerization of the main units affect the energy dissipation rates?

- Does the disorder of the system play a functional role in the energy dissipation? Are excitations mobile or do they relax locally?

-What is the primary mechanism of the energy dissipation in eumelanin?

The application of the two different ultrafast spectroscopy methods on eumelanin and its key building block DHICA was able to answer these questions.

\section{The role of the of the key building blocks in energy dissipation}

The key building block DHICA was extensively studied in Chapter 3 of this thesis to perform a bottom-up approach for addressing eumelanin complex dynamics. It was revealed that the previous studies of this material did not take into account its high oxidizing abilities. The role of the oxygen in excited state deactivation of DHICA demonstrated that under aerated conditions this building block is turning 
into an aggregate, that is not capable of proton transfer, which is reflected in slow energy dissipation. The reason behind that slow decay is that when going through uncontrolled polymerization, DHICA in its monoanion form (which is prevalent in water) forms a high negative charge barrier that impedes proton transfer.

When prepared and measured in the right conditions, DHICA monomers in degassed water exhibit ultrafast excited state decay with the lifetime of 4.25 ps. This lifetime was assigned to the excited state proton transfer from $\mathrm{OH}$ groups to the solvent. The excited state intramolecular proton transfer was studied in DHICA in acidic conditions. It was discovered that its role in the excited state deactivation of DHICA is not important as upon deprotonation of the carboxylic acid group there was no change in the excited state decay rate. Förster analysis of DHICA monomer revealed that in acidic conditions the proton transfer occurs from the carboxylic acid group and not from the $\mathrm{OH}$ group. Hence, the rate of the excited state intramolecular proton transfer is roughly the same as that of excited state proton transfer to the solvent.

Excited state proton transfer from $\mathrm{OH}$ groups to the solvent was proven to be a primary energy dissipation pathway in DHICA monomers. We studied the samples of DHICA in different conditions to test the proton transfer hypothesis. We discovered that the deprotonated form of DHICA in basic water exhibits slow excited state decay in nanosecond timescale, compared with that in methanol, where proton transfer is suppressed. The calculations of the Förster cycle of DHICA proton transfer mechanism demonstrated that DHICA loses a lot of energy through internal conversion, and there is $0.3 \mathrm{eV}$ excess driving force to perform proton transfer. Since the excited state of DHICA in neutral water is on a picosecond timescale, we propose that this form of DHICA is able to access a surface crossing to rapidly get back to the ground state.

The ultrafast character of the UV energy dissipation in the DHICA monomer signifies its importance for the excited state deactivation in eumelanin. 


\section{The effect of oligomerization of the main units on the energy dissi- pation rates}

Chapter 4 of this thesis compares two ways of DHICA oligomerization and its influence on the excited state deactivation. Transient absorption spectroscopy of the synthesized DHICA dimer revealed that the excited state lifetime does not change through the oligomerization process from monomer to dimer. This disproves the previous claim that DHICA dimers possess a much faster excited state decay and hence represent the main functional unit of eumelanin. Our studies hint that the photophysics of the pigment is reflected by the DHICA monomer.

The uncontrolled oxidative polymerization process leads to the prolongation of the excited state decay in DHICA. In neutral conditions oxidatively polymerized mono-anion DHICA oligomers experience decay of $\sim 500$ ps. However, in acidic conditions, where DHICA is in its neutral form, DHICA oligomers and monomers experience similar decay. In acidic conditions, the carboxylic acid group is protonated and hence DHICA has no charge, unlike in neutral water. Therefore, we propose that in neutral water high negative charge density creates a barrier that impedes proton transfer, which results in slow excited state decay. The similarity between DHICA monomer and oligomer in acidic conditions serves as another evidence of the importance of DHICA monomer for eumelanin photophysics. This implies that DHICA monomer, and not dimer, like was proposed earlier, represents eumelanin's excited state behaviour.

\section{Functional role of the disorder of the system in the energy dissipa- tion of eumelanin}

The photophysics of eumelanin was a subject in Chapter 5. By studying the dynamic role of the disorder with the help of fluence and excitation dependent transient absorption spectroscopy we demonstrated that the excitations in eumelanin are localized and confined to $<2.4 \mathrm{~nm}$ radius. The energy dissipation is hence localized in the chromophore that is being excited.

The hypothesis about the formation of the immobile charge transfer states was 
disproved by the study of the DHICA monomer. We demonstrated that the DHICA monomer, which is unable to form a CT state, is exhibiting polarization anisotropy of the PIA band. This concluded that the depolarization of the PIA band did not result from the immobile CT state formation, but is an intrinsic property of the DHICA building block. Instead, the depolarization of the PIA band was claimed to result from the excitation of the ensemble of chromophores that are redistributed within the pulse, causing rapid depolarization. This hypothesis was supported by computational modelling of the eumelanin spectral shape. It was discovered that the chromophores that redistributed within the pulse all have the GSB and PIA peaks at different spectral regions and the GSB and PIA peaks are coming from the different chromophores. The middle plateau resulted from the GSB and PIA of various chromophores, overlapping and cancelling each other out.

\section{The primary mechanism of the energy dissipation in eumelanin}

Eumelanin pigment and DHICA monomer demonstrated a close similarity in their excited state behaviour. The excited state decay lifetime of eumelanin was calculated to be 4.27 ps which is in very good agreement with that of DHICA monomer. The resemblance of the two lifetimes reveals the similarity of the photophysics between these two systems. The proton transfer driving force is expected to be lower for eumelanin chromophores than for DHICA monomers since their excited state energies are lower, however without knowing eumelanin secondary structure it is impossible to perform Förster calculations.

DHICA monomer was proved to have excited state proton transfer as its primary excited state deactivation mechanism. The close similarity between the photophysics of DHICA and eumelanin makes us conclude that the main energy dissipation mechanism in eumelanin is the excited state proton transfer from phenol groups to the solvent. 


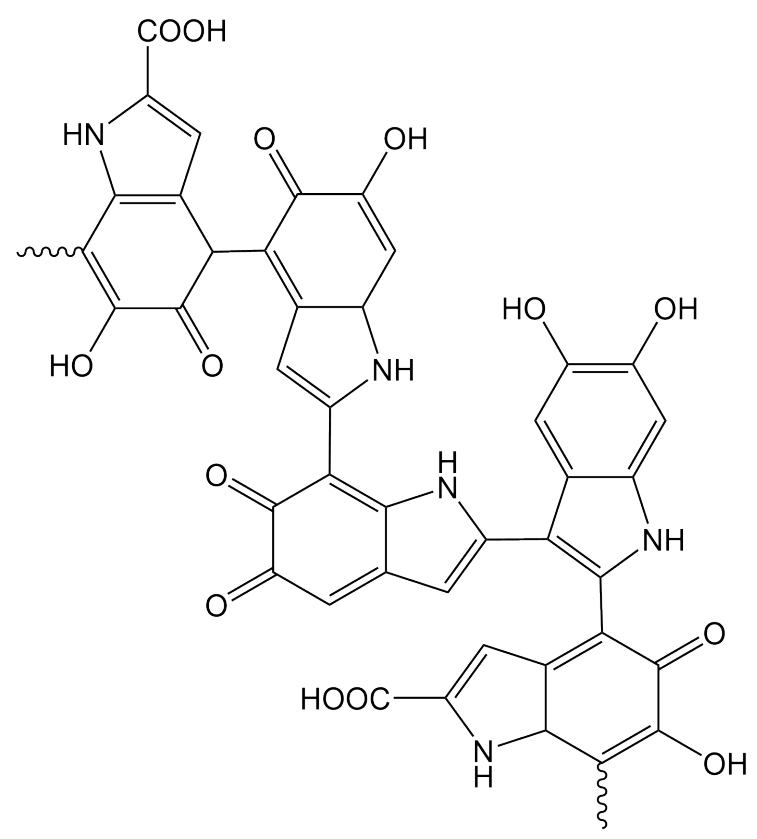

Figure 6.1: The proposed oligomeric structure, containing both DHI and DHICA in different redox forms.

\section{Future work}

The role of DHI monomer and oligomers was not studied in this thesis due to the sample fast degradation. However, eumelanin contains the aggregates comprised of DHICA and DHI in their different forms of oligomerization. The study of the role of DHI in the excited state deactivation of eumelanin would be the last piece to close the existing knowledge gap.

To study how DHI affects the excited state behaviour in different oligomers, we can synthesize oligomers with different DHICA/DHI ratios. This work will provide an idea of the importance of each building block in eumelanin photophysics. By synthesizing those oligomers we would be able to represent different eumelanin chromophores.

The role of quinone forms was not acknowledged in this work, since these oxidized forms of the main building blocks are hard to synthesize. However, eumelanin contains DHICA and DHI and their various quinone forms. The oligomers 


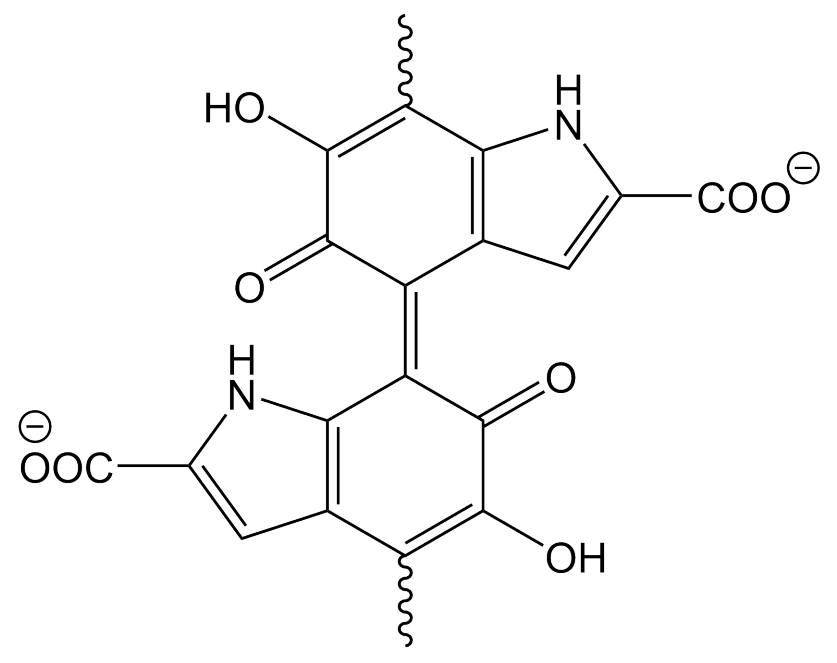

Figure 6.2: The proposed incorporation of DHICA quinones to study the oxidative polymerization.

of DHICA and DHI in different redox formations would produce chromophores of various lengths and HOMO-LUMO gaps, covering the entire range of the absorption of eumelanin. Obtaining those structures for Förster analysis would be an important step in understanding the role of proton transfer in eumelanin aggregates. The possible structure to study is proposed in Figure 6.1.

Additionally, the quinone forms might affect the excited state decay rate of the DHICA molecules in aerated conditions. The incorporation of quinones is assumed to modify the molecular conformation and alter its ability to perform proton transfer. Hence, the synthesis of the oligomers, containing all quinone forms might add up to the knowledge about the processes in DHICA in aerated conditions. The potential conformational locking of the structure due to the quinones incorporations is proposed in Figure 6.2.

In order to address the question about the main functional unit in eumelanin energy dissipation, the more thorough study of DHICA oligomers is needed. The synthesis of DHICA trimers, tetramers and DHICA-melanin will reveal whether the excited state of DHICA oligomers is still not affected through polymerization. The similar study of DHI could also be possible to reveal the true role of each of the building blocks and their "pure" oligomers. 


\section{Appendix A}

\section{Matlab code used for energy transfer simulations in multichromophore system}

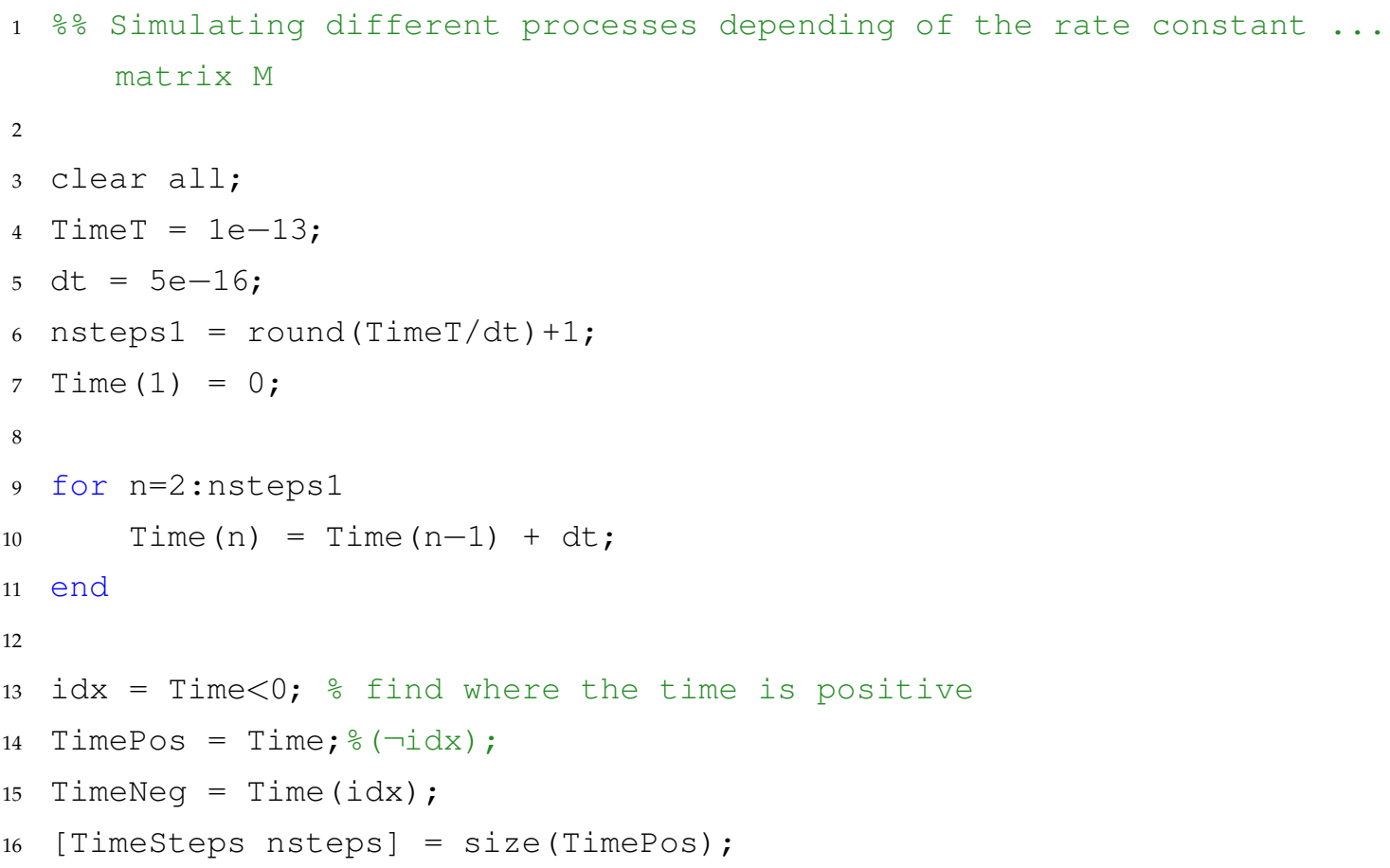




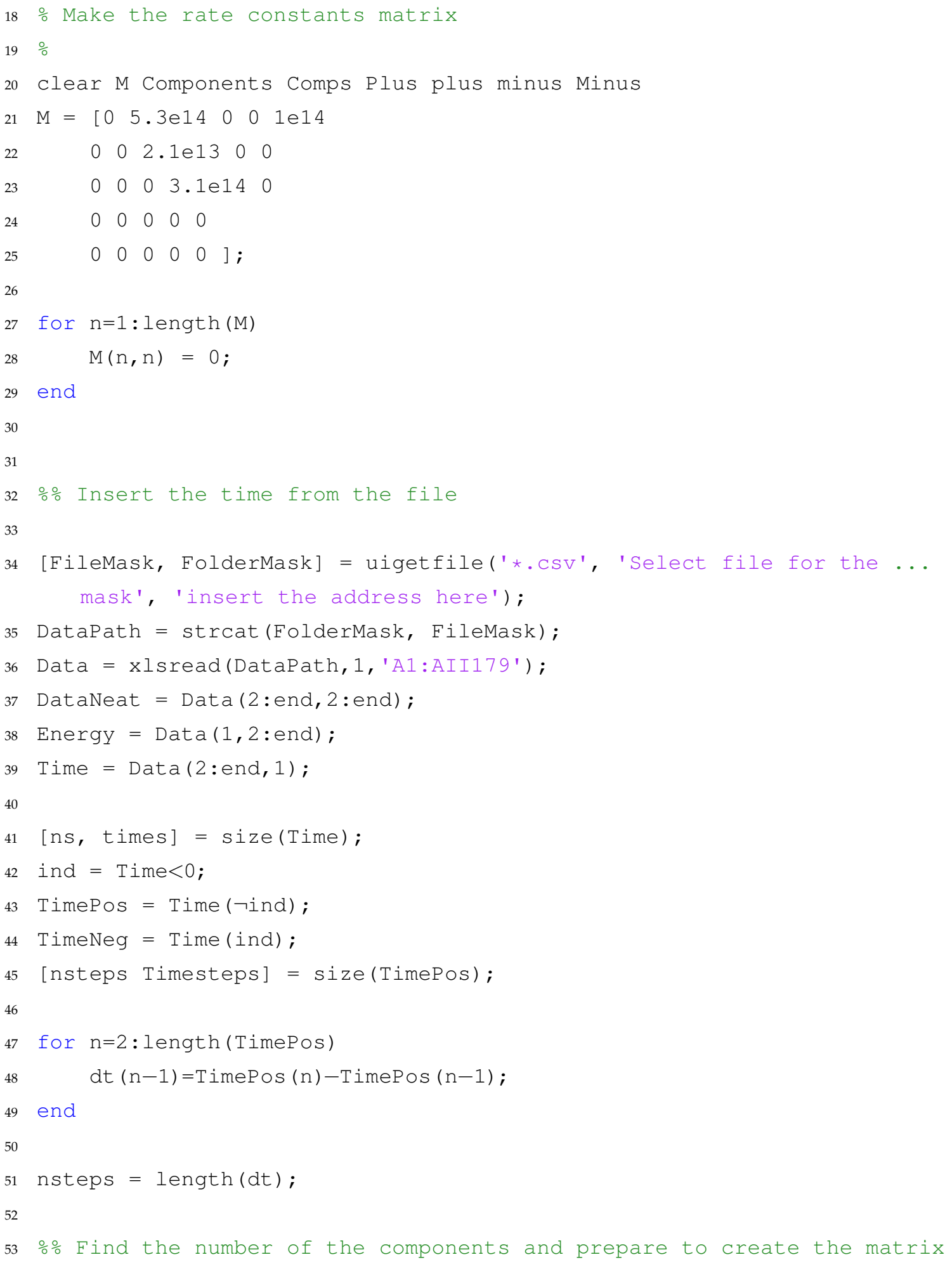


$[r, \mathrm{C}]=\operatorname{size}(\mathrm{M})$;

56 NumComponents $=\mathrm{C}$;

57 Components $=$ zeros (NumComponents, length(TimePos));

58 


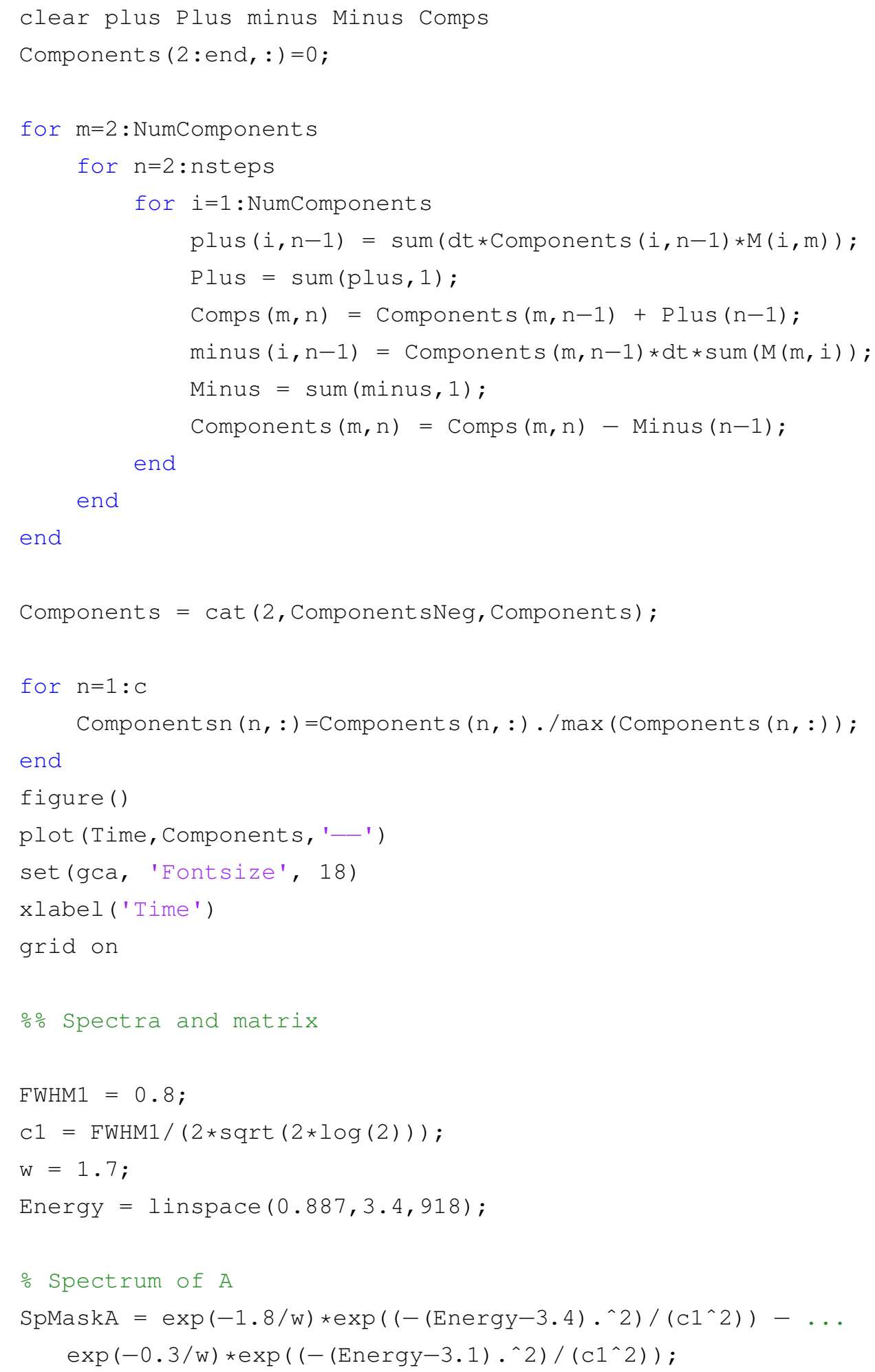




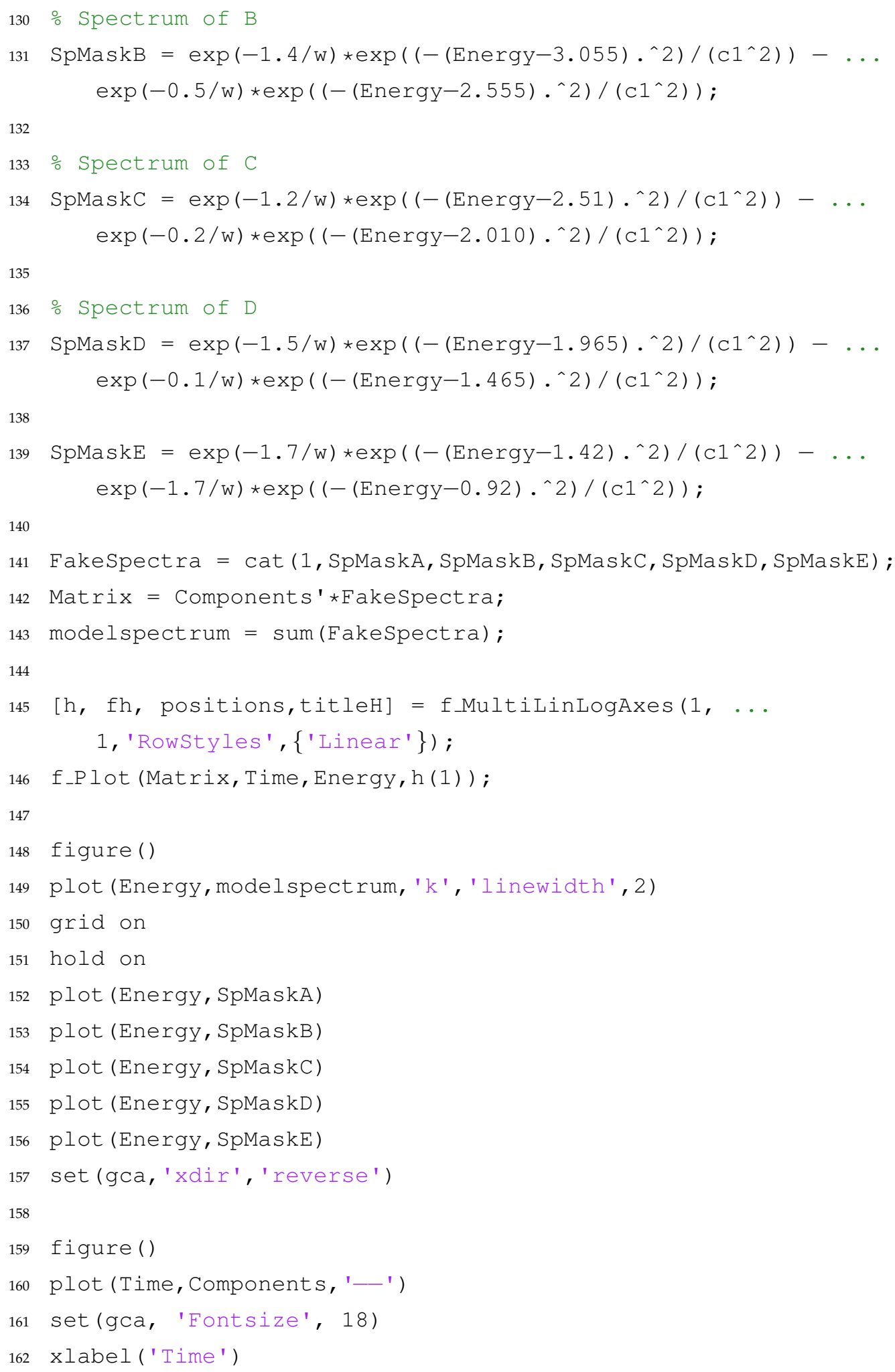


163 grid on

164

165 figure ()

166 plot (Energy, Fakespectra, 'Linewidth ', 2)

167 set (gca, 'Fontsize', 18)

168 set (gca, 'XDir', 'Reverse')

169 xlabel ('Energy, eV')

170 grid on 


\section{Bibliography}

[1] H. Abramczyk. Ultrafast Chemical and Physical Processes. 2005.

[2] Govind P Agrawal, PL Baldeck, and RR Alfano. Temporal and spectral effects of cross-phase modulation on copropagating ultrashort pulses in optical fibers. Physical Review A, 40(9):5063, 1989.

[3] Tagreed M Al-Saadi, Bushra H Hussein, Alaa B Hasan, and AA Shehab. Study the structural and optical properties of cr doped sno2 nanoparticles synthesized by sol-gel method. Energy Procedia, 157:457-465, 2019.

[4] Robert R. Alfano. The ultimate white light. Scientific American, 295(6):86-93, 2006.

[5] RR Alfano and SL Shapiro. Emission in the region 4000 to 7000 å via four-photon coupling in glass. Physical Review Letters, 24(11):584, 1970.

[6] RR Alfano and SL Shapiro. Observation of self-phase modulation and small-scale filaments in crystals and glasses. Physical Review Letters, 24(11):592, 1970.

[7] Antonio Aloi, Adalberto Brunetti, Giuseppe Perna, Maria Lasalvia, Vito Capozzi, and Raffaele Tommasi. The Physical Properties of Melanins. Biomedical Optics Express, 6:4000-4013, 2015.

[8] Andreas P Arndt, Marina Gerhard, Martin Koch, Uli Lemmer, and Ian A Howard. Identifying charge-transfer states in polymer: fullerene heterojunctions by their emission polarization anisotropy. The Journal of Physical Chemistry C, 121(11):6357-6364, 2017.

[9] S Arzhantsev and Mark Maroncelli. Design and characterization of a femtosecond fluorescence spectrometer based on optical kerr gating. Applied spectroscopy, 59(2):206-220, 2005.

[10] PL Baldeck, PP Ho, and Robert R Alfano. Cross-phase modulation: a new technique for controlling the spectral, temporal, and spatial properties of ultrashort pulses. In The Supercontinuum Laser Source, pages 117-183. Springer, 1989. 
[11] Prakriti Ranjan Bangal and Sankar Chakravorti. Excited state proton transfer in indole-2carboxylic acid and indole-5-carboxylic acid. The Journal of Physical Chemistry A, 103(43):85858594, 1999.

[12] Paul S Banks, Michael D Feit, and Michael D Perry. High-intensity third-harmonic generation. JOSA B, 19(1):102-118, 2002.

[13] ALEX J. BARKER, KAI CHEN, SHYAMAL K.K. PRASAD, and JUSTIN M. HODGKISS. HighSensitivity Ultrafast Transient Absorption Spectroscopy of Organic Photovoltaic Devices. Ultrafast Dynamics in Molecules, Nanostructures and Interfaces, pages 36-52, 2014.

[14] A. Bernardus Mostert, Ben J. Powell, Ian R. Gentle, and Paul Meredith. On the origin of electrical conductivity in the bio-electronic material melanin. Applied Physics Letters, 100(9):1-4, 2012.

[15] SJ Blundell. Spin-polarized muons in condensed matter physics. Contemporary Physics, 40(3):175-192, 1999.

[16] Jacques P. Bothma, Johannes De Boor, Ujjual Divakar, Paul E. Schwenn, and Paul Meredith. Device-quality electrically conducting melanin thin films. Advanced Materials, 20(18):3539$3542,2008$.

[17] Robert W Boyd. Nonlinear optics. Academic press, 2020.

[18] Maximilian Bradler, Peter Baum, and Eberhard Riedle. Femtosecond continuum generation in bulk laser host materials with sub- $\mu$ j pump pulses. Applied Physics B, 97(3):561-574, 2009.

[19] F. Bray, J. Ferlay, I. Soerjomataram, R. L. Siegel, L. A. Torre, and A. Jemal. Skin cancer statistics. melanoma of the skin is the 19th most common cancer worldwide. https://www.wcrf.org/ dietandcancer/cancer-trends/skin-cancer-statistics, 2018.

[20] Michaela Brenner and Vincent J. Hearing. The protective role of melanin against UV damage in human skin. Photochemistry and Photobiology, 84(3):539-549, 2008.

[21] Michela Brenner and Vincent J. Hearing. NIH Public Access. Cancer, 84(3):539-549, 2009.

[22] J. Brian Nofsinger and John D. Simon. Radiative Relaxation of Sepia Eumelanin is Affected by AggregationII. Photochemistry and Photobiology, 74(1):31-37, 2007.

[23] A Brodeur and SL Chin. Ultrafast white-light continuum generation and self-focusing in transparent condensed media. JOSA B, 16(4):637-650, 1999. 
[24] Carmella Calabrese, Ashley M Stingel, Lei Shen, and Poul B Petersen. Ultrafast continuum mid-infrared spectroscopy: probing the entire vibrational spectrum in a single laser shot with femtosecond time resolution. Optics letters, 37(12):2265-2267, 2012.

[25] E.F. Caldin and V. Gold. Proton-trasfer reactions. 1975.

[26] Mojca Cepic. Direction dependency of extraordinary refraction index. arXiv preprint physics/0108013, 2001.

[27] Giulio Cerullo and Sandro De Silvestri. Ultrafast optical parametric amplifiers. Review of scientific instruments, 74(1):1-18, 2003.

[28] Sreelakshmi Chandrabose, Kai Chen, Alex J Barker, Joshua J Sutton, Shyamal KK Prasad, Jingshuai Zhu, Jiadong Zhou, Keith C Gordon, Zengqi Xie, Xiaowei Zhan, et al. High exciton diffusion coefficients in fused ring electron acceptor films. Journal of the American Chemical Society, 141(17):6922-6929, 2019.

[29] Louise K Charkoudian and Katherine J Franz. Fe (iii)-coordination properties of neuromelanin components: 5, 6-dihydroxyindole and 5, 6-dihydroxyindole-2-carboxylic acid. Inorganic Chemistry, 45(9):3657-3664, 2006.

[30] Chun Teh Chen, Chern Chuang, Jianshu Cao, Vincent Ball, David Ruch, and Markus J. Buehler. Excitonic effects from geometric order and disorder explain broadband optical absorption in eumelanin. Nature Communications, 5(May):1-10, 2014.

[31] Kai Chen, Joseph K Gallaher, Alex J Barker, and Justin M Hodgkiss. Transient grating photoluminescence spectroscopy: an ultrafast method of gating broadband spectra. The journal of physical chemistry letters, 5(10):1732-1737, 2014.

[32] J Cheng, S C Moss, and M Eisner. X-Ray Characterization of Melanins .2. Pigment Cell Research, 7(4):263-273, 1994.

[33] J Cheng, S C Moss, M Eisner, and P Zschack. X-Ray Characterization of Melanins .1. Pigment Cell Research, 7(4):255-262, 1994.

[34] Orlando Chiarelli-Neto, Alan Silva Ferreira, Waleska Kerllen Martins, Christiane Pavani, Divinomar Severino, Fernanda Faião-Flores, Silvya Stuchi Maria-Engler, Eduardo Aliprandini, Glaucia R. Martinez, Paolo Di Mascio, Marisa H.G. Medeiros, and Maurício S. Baptista. Melanin photosensitization and the effect of visible light on epithelial cells. PLoS ONE, 9(11):19, 2014. 
[35] Pi-Tai Chou and Shannon Studer Martinez. Reinvestigation of solvent catalyzed ground-state reverse proton transfer in 7-hydroxyquinoline. Chemical physics letters, 235(5-6):463-470, 1995.

[36] Christine M. R. Clancy and John D. Simon. Ultrastructural Organization of Eumelanin from Sepia officinalis Measured by Atomic Force Microscopy . Biochemistry, 40:13353, 2001.

[37] Alice Corani, Annemarie Huijser, Thomas Gustavsson, Dimitra Markovitsi, Per Åke Malmqvist, Alessandro Pezzella, Marco D'Ischia, and Villy Sundström. Superior photoprotective motifs and mechanisms in eumelanins uncovered. Journal of the American Chemical Society, 136(33):11626-11635, 2014.

[38] Alice Corani, Alessandro Pezzella, Torbjörn Pascher, Thomas Gustavsson, Dimitra Markovitsi, Annemarie Huijser, Marco D'Ischia, and Villy Sundström. Excited-state proton-transfer processes of DHICA resolved: From sub-picoseconds to nanoseconds. Journal of Physical Chemistry Letters, 4(9):1383-1388, 2013.

[39] Arnaud Couairon and André Mysyrowicz. Femtosecond filamentation in transparent media. Physics reports, 441(2-4):47-189, 2007.

[40] P. R. Crippa, V. Cristofoletti, and N. Romeo. A band model for melanin deduced from optical absorption and photoconductivity measurements. Biochimica et Biophysica Acta, 583:164, 1978.

[41] CH Brito Cruz, JP Gordon, PC Becker, RL Fork, and Charles V Shank. Dynamics of spectral hole burning. IEEE journal of quantum electronics, 24(2):261-269, 1988.

[42] Jayashree A Dharmadhikari, Rucha A Deshpande, Arpita Nath, Krithika Dota, Deepak Mathur, and Aditya K Dharmadhikari. Effect of group velocity dispersion on supercontinuum generation and filamentation in transparent solids. Applied Physics B, 117(1):471-479, 2014.

[43] Marco d'Ischia, Alessandra Napolitano, Alessandro Pezzella, Paul Meredith, and Tadeusz Sarna. Chemical and structural diversity in eumelanins: unexplored bio-optoelectronic materials. Angewandte Chemie International Edition, 48(22):3914-3921, 2009.

[44] R Dorsinville, PP Ho, JT Manassah, and RR Alfano. Applications of supercontinuum: present and future. In The supercontinuum laser source, pages 377-398. Springer, 1989.

[45] Abderrazzak Douhal, Fracoise Lahmani, and Ahmed H. Zewail. Proton-transfer reaction dynamics. Chemical Physics, 207:477-498, 1996.

[46] Daniel R. Dreyer, Daniel J. Miller, Benny D. Freeman, Donald R. Pual, and Christopher W. Bielawski. Elucidating the Structure of Poly(dopamine). Langmuir, 28:6428-6435, 2012. 
[47] Audrius Dubietis and Arnaud Couairon. Ultrafast supercontinuum generation in transparent solidstate media. Springer, 2019.

[48] Audrius Dubietis, Gintaras Tamošauskas, Rosvaldas Šuminas, Vytautas Jukna, and Arnaud Couairon. Ultrafast supercontinuum generation in bulk condensed media. 2017.

[49] David Eimerl, L Davis, Stephan Velsko, EK Graham, and A Zalkin. Optical, mechanical, and thermal properties of barium borate. Journal of applied physics, 62(5):1968-1983, 1987.

[50] K Ekvall, P Van Der Meulen, C Dhollande, L-E Berg, S Pommeret, R Naskrecki, and J-C Mialocq. Cross phase modulation artifact in liquid phase transient absorption spectroscopy. Journal of applied physics, 87(5):2340-2352, 2000.

[51] E. Engel, K. Leo, and M. Hoffmann. Ultrafast relaxation and exciton-exciton annihilation in PTCDA thin films at high excitation densities. Chemical Physics, 325:170-177, 2006.

[52] W. S. Enochs, T. Sarna, L. Zecca, P. A. Riley, and H. M. Swartz. The roles of neuromelanin, binding of metal ions, and oxidative cytotoxicity in the pathogenesis of parkinson's disease: a hypothesis. Journal of neural transmission-Parkinson's disease and dementia section, 7(2):83-100, 1994.

[53] Henrik Enqvist. A setup for efficient frequency tripling of high-power femtosecond laser pulses. Lund reports, (October):45, 2004.

[54] H. Fedorow, F. Tribl, G. Halliday, M. Gerlach, P. Riederer, and K. L. Double. Neuromelanin in human dopamine neurons: comparison with peripheral melanins and relevance to parkinson's disease. Progress in neurobiology, 75(2):109-124, 2005.

[55] Susan E Forest and John D Simon. Wavelength-dependent photoacoustic calorimetry study of melanin. Photochemistry and photobiology, 68(3):296-298, 1998.

[56] Sebastião J Formosinho and Luís G Arnaut. Excited-state proton transfer reactions ii. intramolecular reactions. Journal of Photochemistry and Photobiology A: Chemistry, 75(1):21-48, 1993.

[57] Th Förster. Die ph-abhängigkeit der fluoreszenz von naphthalinderivaten. Zeitschrift für Elektrochemie und angewandte physikalische Chemie, 54(7):531-535, 1950.

[58] Patrick Frank and PPE General. Schlenk line design and safety, 2011.

[59] J M Gallas, M Eisner, and S C Moss. Bioch . 1199:271-278, 1994. 
[60] M. Gauden, A. Pezzella, L. Panzella, M. T. Neves-Petersen, E. Skovsen, S. B. Petersen, K. M. Mullen, A. Napolitano, M. D'Ischia, and V. Sundström. Role of solvent, $\mathrm{pH}$, and molecular size in excited-state deactivation of key eumelanin building blocks: Implications for melanin pigment photostability. Journal of the American Chemical Society, 130(50):17038-17043, 2008.

[61] Magdalena Gauden, Alessandro Pezzella, Lucia Panzella, Alessandra Napolitano, Marco Ischia, and Villy Sundstrom. Ultrafast Excited State Dynamics of 5 , 6-Dihydroxyindole, A Key Eumelanin Building Block: Nonradiative Decay Mechanism. page 12575, 2009.

[62] B. A. Gilchrest, M. S. Eller, A. C. Geller, and M. Yaar. The pathogenesis of melanoma induced by ultraviolet radiation. . N Engl J Med., 340:1341, 1998.

[63] EN Glezer, Y Siegal, L Huang, and E Mazur. Laser-induced band-gap collapse in gaas. Physical Review B, 51(11):6959, 1995.

[64] GLOBOCAN. Cancer today. https://gco.iarc.fr/today, 2021. [Online; accessed 11January-2021].

[65] V. P. Grishchuk, S. A. Davidenko, I. D. Zholner, A. B. Verbitskii, M. V. Kurik, and Piryatinskii Y. P. Optical absorption and luminescent properties of melanin films. Technical Physics Letters, 28:36, 2002.

[66] S Haacke, RA Taylor, I Bar-Joseph, MJSP Brasil, M Hartig, and B Deveaud. Improving the signal-to-noise ratio of femtosecond luminescence upconversion by multichannel detection. JOSA B, 15(4):1410-1417, 1998.

[67] Lawrence J Henderson. Concerning the relationship between the strength of acids and their capacity to preserve neutrality. American Journal of Physiology-Legacy Content, 21(2):173-179, 1908.

[68] H. Z. Hill and G. J. Hill. UVA, pheomelanin and the carcinogenesis of melanoma . Pigment Cell Research, 13:140, 2000.

[69] Helene Z Hill, Weixjiong Li, Patrick Xin, and David L Mitchell. Melanin: a two edged sword? Pigment cell research, 10(3):158-161, 1997.

[70] Justin M Hodgkiss, Sebastian Albert-Seifried, Akshay Rao, Alex J Barker, Andrew R Campbell, R Alex Marsh, and Richard H Friend. Exciton-charge annihilation in organic semiconductor films. Advanced Functional Materials, 22(8):1567-1577, 2012.

[71] J Michael Hollas. Modern spectroscopy. John Wiley \& Sons, 2004. 
[72] Erin Hopkins, Terrence Sanvictores, and Sandeep Sharma. Physiology, acid base balance. StatPearls [Internet], 2020.

[73] Annemarie Huijser, Alessandro Pezzella, Jonas K. Hannestad, Lucia Panzella, Alessandra Napolitano, Marco d'Ishia, and Villy Sundstrom. UV-Dissipation Mechanisms in the Eumelanin Building Block DHICA. ChemPhysChem, 11:2424-2431, 2010.

[74] Annemarie Huijser, Alessandro Pezzella, and Villy Sundström. Functionality of epidermal melanin pigments: Current knowledge on UV-dissipative mechanisms and research perspectives. Physical Chemistry Chemical Physics, 13(20):9119-9127, 2011.

[75] Annemarie Huijser, Michał F Rode, Alice Corani, Andrzej L Sobolewski, and Villy Sundström. Photophysics of indole-2-carboxylic acid in an aqueous environment studied by fluorescence spectroscopy in combination with ab initio calculations. Physical chemistry chemical physics, 14(6):2078-2086, 2012.

[76] Shosuke Ito and Kazumasa Wakamatsu. Quantitative analysis of eumelanin and pheomelanin in humans, mice, and other animals: a comparative review. Pigment cell research, 16(5):523-531, 2003.

[77] Shousuke Ito. A Chemist's View of Melanogenesis. Pigment Cell Res., 16, 2003.

[78] J. E. McGinness. Mobility Gaps: A Mechanism For Band Gaps in Melanins. Science, 177(4052):896-897, 1972.

[79] David M Jameson and Justin A Ross. Fluorescence polarization/anisotropy in diagnostics and imaging. Chemical reviews, 110(5):2685-2708, 2010.

[80] R Jankowiak, JM Hayes, and GJ Small. Spectral hole-burning spectroscopy in amorphous molecular solids and proteins. Chemical reviews, 93(4):1471-1502, 1993.

[81] R Jankowiak and GJ Small. Hole-burning spectroscopy and relaxation dynamics of amorphous solids at low temperatures. Science, 237(4815):618-625, 1987.

[82] Maria M. Jastrzebska, Heikki Isotalo, Jari Paloheimo, and Henrik Stubb. Electrical conductivity of synthetic DOPA-melanin polymer for different hydration states and temperatures. Journal of Biomaterials Science, Polymer Edition, 7(7):577-586, 1995.

[83] Joaquim Jaumot, Raimundo Gargallo, Anna de Juan, and Roma Tauler. A graphical userfriendly interface for mcr-als: a new tool for multivariate curve resolution in matlab. Chemometrics and intelligent laboratory systems, 76(1):101-110, 2005. 
[84] David M Jonas, Matthew J Lang, Yutaka Nagasawa, Taiha Joo, and Graham R Fleming. Pumpprobe polarization anisotropy study of femtosecond energy transfer within the photosynthetic reaction center of rhodobacter sphaeroides r26. The Journal of Physical Chemistry, 100(30):1266012673, 1996.

[85] Valeriy Petrovich Kandidov, Svyatoslav A Shlenov, and Ol'ga Grigor'evna Kosareva. Filamentation of high-power femtosecond laser radiation. Quantum Electronics, 39(3):205, 2009.

[86] Michael Kasha. Characterization of electronic transitions in complex molecules. Discussions of the Faraday society, 9:14-19, 1950.

[87] Efthimios Kaxiras, Argyrios Tsolakidis, George Zonios, and Sheng Meng. Stuctural Model of Eumelanin. Physical Review Letters, 97(218102), 2006.

[88] Z. Khazaei, F. Ghorat, A. M. Jarrahi, H. A. Adineh, M. Sohrabivafa, and E. Goodariz. Global incidence and mortality of skin cancer by histological subtyp anf its relationship with the human development index (HDI); and ecology study in 2018 . World cancer research journal, 6:1, 2018.

[89] In-Sik Kim, Byoung-uk Sohn, Sung In Hwang, Chang-Lyoul Lee, and Do-Kyeong Ko. Chirpindependent time-resolved spectroscopy using the self-reference method. Laser Physics, 24(4):045701, 2014.

[90] M. Klessinger and J. Michl. Excited States and Photochemistry of Organic Molecules. VCH: New York, 1995.

[91] N. Kobayashi, A. Nakagawa, T. Muramatsu, Y. Yamashina, T. Shirai, M. W. Hashimoto, Y. Ishigaki, T. Ohnishi, and T. Mori. Supranuclear melanin caps reduce ultraviolet induced DNA photoproducts in human epidermis . J. Invest. Dermatol., 110:806, 1998.

[92] Forrest R Kohl, Christopher Grieco, and Bern Kohler. Ultrafast spectral hole burning reveals the distinct chromophores in eumelanin and their common photoresponse. Chemical Science, 11(5):1248-1259, 2020.

[93] Nikiforos Kollias, Robert M Sayre, Lisa Zeise, and Miles R Chedekel. New trends in photobiology: Photoprotection by melanin. Journal of Photochemistry and Photobiology B: Biology, 9(2):135-160, 1991.

[94] Edward M Kosower and Dan Huppert. Excited state electron and proton transfers. Annual Review of Physical Chemistry, 37(1):127-156, 1986. 
[95] Philipp Kukura, David W McCamant, and Richard A Mathies. Femtosecond stimulated raman spectroscopy. Annu. Rev. Phys. Chem., 58:461-488, 2007.

[96] Joseph R Lakowicz. Principles of fluorescence spectroscopy. Springer science \& business media, 2013.

[97] JR Lakowicz. Principles of fluoresence spectroscopy. kluwer. New York, 1999.

[98] L. D. Landau and E.M. Lifshitz. Electrodynamics of Continuous Media. Vol. 8 [in Russian]. FISMALIT, 2005.

[99] John Lekner. Reflection and refraction by uniaxial crystals. Journal of Physics: Condensed Matter, 3(32):6121, 1991.

[100] Y Liu and J D Simon. Isolation and biophysical studies of natural eumelanins: Applications of imaging technologies and ultrafast spectroscopy [Review]. Pigment Cell Research, 16(6):606618, 2003.

[101] H. C. Longuet-Higgins. On the origin of the free radical property of melanins. Archives of Biochemistry and Biophysics, 86(2):231-232, 1960.

[102] M. Lorenc, M. Ziolek, R. Naskrecki, J. Karolczak, J. Kubicki, and A Maciejewski. Artifacts in femtosecond transient absorption spectroscopy. Applied Physics B, 74(1):19-27, 2002.

[103] Haibo Ma and Alessandro Troisi. Direct optical generation of long-range charge-transfer states in organic photovoltaics. Advanced Materials, 26(35):6163-6167, 2014.

[104] Ian H Malitson. Interspecimen comparison of the refractive index of fused silica. Josa, 55(10):1205-1209, 1965.

[105] V. May. Kinetic theory of exciton-exciton annihilation. J. Chem. Phys., 140:054103, 2014.

[106] David W McCamant, Philipp Kukura, and Richard A Mathies. Femtosecond broadband stimulated raman: a new approach for high-performance vibrational spectroscopy. Applied spectroscopy, 57(11):1317-1323, 2003.

[107] David W McCamant, Philipp Kukura, Sangwoon Yoon, and Richard A Mathies. Femtosecond broadband stimulated raman spectroscopy: Apparatus and methods. Review of scientific instruments, 75(11):4971-4980, 2004.

[108] John Mcginness, Peter Corry, and Peter Proctor. Amorphous semiconductor switching in melanins. Science, 183(4127):853-855, 1974. 
[109] U. Megerle, I. Pugliesi, C. Schriever, C.F. Sailer, and E. Riedle. Sub-50 fs broadband absorption spectroscopy with tunable excitation: putting the analysis of ultrafast molecular dynamics on solid ground. Appl.Phys.B, 96:215-231, 2009.

[110] Sheng Meng and Efthimios Kaxiras. Mechanisms for ultrafast nonradiative relaxation in electronically excited eumelanin constituents. Biophysical Journal, 95(9):4396-4402, 2008.

[111] Paul Meredith, Ben J. Powell, Jennifer Riesz, Stephen P. Nighswander-Rempel, Mark R. Pederson, and Evan G. Moore. Towards structure-property-function relationships for eumelanin. Soft Matter, 2(1):37-44, 2006.

[112] Paul Meredith and Jennifer Riesz. Radiative Relaxation Quantum Yields for Synthetic EumelaninI. Photochemistry and Photobiology, 79(2):211, 2004.

[113] Paul Meredith and Tadeusz Sarna. The physical and chemical properties of eumelanin. Pigment Cell Res., 19:572-594, 2006.

[114] Rubens S Miranda, Gloria R Jacobovitz, Carlos H Brito Cruz, and Marco AF Scarparo. Positive and negative chirping of laser pulses shorter than $100 \mathrm{fsec}$ in a saturable absorber. Optics letters, 11(4):224-226, 1986.

[115] Willard L Miranker. Numerical methods of boundary layer type for stiff systems of differential equations. Computing, 11(3):221-234, 1973.

[116] A. B. Mostert, J. L. Davy, Ruggles J. L., B. J. Powell, I. R. Gentle, and P. Meredith. Gaseous Adsorption in Melanins: Hydrophilic Biomacromolecules with High Electrical Conductivities. Langmuir, 26:412, 2010.

[117] A. B. Mostert, B. J. Powell, F. L. Pratt, G. R. Hanson, T. Sarna, I. R. Gentle, and P. Meredith. Role of semiconductivity and ion transport in the electrical conduction of melanin. Proceedings of the National Academy of Sciences, 109(23):8943-8947, 2012.

[118] R Nakamura and Y Kanematsu. Femtosecond spectral snapshots based on electronic optical kerr effect. Review of scientific instruments, 75(3):636-644, 2004.

[119] Ryszard Naskrecki, Marjorie Ménard, Peter van der Meulen, Georges Vigneron, and Stanislas Pommeret. Three-photon absorption cross-section of simple molecular liquids. Optics communications, 153(1-3):32-38, 1998.

[120] ETJ Nibbering, PF Curley, Georges Grillon, BS Prade, MA Franco, François Salin, and André Mysyrowicz. Conical emission from self-guided femtosecond pulses in air. Optics letters, 21(1):62-64, 1996. 
[121] Stephen P. Nighswander-Rempel, Indumathy B. Mahadevan, Halina Rubinsztein-Dunlop, and Paul Meredith. Time-resolved and steady-state fluorescence spectroscopy of eumelanin and indolic polymers. Photochemistry and Photobiology, 83(6):1449-1454, 2007.

[122] Stephen P. Nighswander-Rempel, Jennifer Riesz, Joel Gilmore, Jacques P. Bothma, and Paul Meredith. Quantitative Fluorescence Excitation Spectra of Synthetic Eumelanin. J. Phys. Chem. B, 109(43):20629-20635, 2005.

[123] Stephen P. Nighswander-Rempel, Jennifer Riesz, Joel Gilmore, and Paul Meredith. A quantum yield map for synthetic eumelanin. Journal of Chemical Physics, 123(19), 2005.

[124] J. Brian Nofsinger, Susan E. Forest, and John D. Simon. Explanation for the Disparity among Absorption and Action Spectra of Eumelanin. J. Phys. Chem. B, 103(51):146-151, 1999.

[125] J Brian Nofsinger and John D Simon. Radiative relaxation of sepia eumelanin is affected by aggregation. Photochemistry and Photobiology, 74(1):31-37, 2001.

[126] J. Brian Nofsinger, Tong Ye, and John D. Simon. Ultrafast Nonradiative Relaxation Dynamics of Eumelanin. J. Phys. Chem. B, 105:2864-2866, 2001.

[127] Juan J. Nogueira, Alice Corani, Amal El Nahhas, Alessandro Pezzella, Marco d'Ishia, Leticia Gonzalez, and Villy Sundstrom. Sequential Proton-Coupled Electron Transfer Mediates Excited-State Deactivation of a Eumelanin Building Block. J. Phys. Chem. Lett., 8:1004-1008, 2017.

[128] Seth Olsen, Jennifer Riesz, Indu Mahadevan, Aaron Coutts, Jacques P. Bothma, Benjamin J. Powell, Ross H. McKenzie, Sean C. Smith, and Paul Meredith. Convergent proton-transfer photocycles violate mirror-image symmetry in a key melanin monomer. Journal of the American Chemical Society, 129(21):6672-6673, 2007.

[129] J-P. Ortonne. Photoprotective properties of skin melanin. British Journal of Dermatology, 146:7$10,2002$.

[130] W. Osak, T. Katarzyna, H. Czternastek, and J. Slawinski. I-V characteristics and electrical conductivity of synthetic melanin. Biopolymers, 28:1885, 1989.

[131] S. S. Ostakhov, V. P. Kazakov, and I. O. Osina. Nature of extrema of the activation energy of temperature quenching of tryptophan fluorescence as a function of the excitation light wavelength [in russian]. volume 423, pages 208-211, 2008.

[132] F. L. Pedrotti and L. S. Pedtrotti. Introduction to Optics, second edition. Prentice Hall, 1996. 
[133] Jorge Peon, Gina C Hess, Jean-Marc L Pecourt, Tetsuro Yuzawa, and Bern Kohler. Ultrafast photoionization dynamics of indole in water. The Journal of Physical Chemistry A, 103(14):24602466, 1999.

[134] Alessandro Pezzella, Alessandra Napolitano, Marco d'Ischia, and Giuseppe Prota. Oxidative polymerisation of 5, 6-dihydroxyindole-2-carboxylic acid to melanin: A new insight. Tetrahedron, 52(23):7913-7920, 1996.

[135] Ehud Pines and Graham R Fleming. Proton transfer in mixed water-organic solvent solutions: Correlation between rate, equilibrium constant, and the proton free energy of transfer. The Journal of Physical Chemistry, 95(25):10448-10457, 1991.

[136] Henry N Po and NM Senozan. The henderson-hasselbalch equation: its history and limitations. Journal of Chemical Education, 78(11):1499, 2001.

[137] B. J. Powell. 5,6-dihydroxyindole-2-carboxylic acid: a first principles density functional study. Chemical Physics Letters, 204:111, 2005.

[138] B. J. Powell, T. Baruah, N. Bernstein, K. Brake, Ross H. McKenzie, P. Meredith, and M. R. Pederson. A first principles density-functional theory calculation of the electronic and vibrational structure of the key melanin monomers. Journal of Chemical Physics, 120:8608, 2004.

[139] Michael R. Powell and Barnett Rosenberg. The nature of the charge carriers in solvated biomacromolecules. Journal of Bioenergetics, 1(6):493-509, 1970.

[140] Michael Price, Paul Hume, Aleksandra Ilina, Isabella Wagner, Ronnie Tamming, Karen Thorn, Wanting Jiao, Alison Campbell, Patrick Conaghan, Girish Lakhwanid, Nathaniel Davis, Kai Chen, Yifan Wang, Peiyao Xue, Heng Lu, Xiaowei Zhan, and Justin Hodgkiss. Free charge photogeneration in a single component high photovoltaic efficiency organic semiconductor. 2021.

[141] G. Prota. Progress in the chemistry of melanins and related metabolites . Medical Research Reviews, 8:525, 1988.

[142] G. Prota. Melanins and Melanogenesis. Academic Press, San Diego, 1992.

[143] A. Pullman and B. Pullman. The band structure of melanins. Biochimica Biophysica Acta, 54:384, 1961.

[144] H. S. Raper. The tyrosinase-tyrosine reaction. VI. production from tyrosine of 5,6dihydroxyindole and 5,6-dihydroxyindole-2-carboxylic acid - the precursors of melanin. . Journal of Biochemistry, 21:89, 1927. 
[145] H. S. Raper. The aerobic oxidases . Physiology Reviews, 8:245, 1928.

[146] Achim Reuther, Alfred Laubereau, and David N Nikogosyan. A simple method for the in situ analysis of femtosecond uv pulses in the pump-probe spectroscopy of solutions. Optics communications, 141(3-4):180-184, 1997.

[147] Jennifer Riesz, Joel Gilmore, and Paul Meredith. Quantitative photoluminescence of broad band absorbing melanins: A procedure to correct for inner filter and re-absorption effects. Spectrochimica Acta - Part A: Molecular and Biomolecular Spectroscopy, 61(9):2153-2160, 2005.

[148] Jennifer Riesz, Joel Gilmore, and Paul Meredith. Quantitative scattering of melanin solutions. Biophysical Journal, 90(11):4137-4144, 2006.

[149] Roger E Rondeau. A technique for degassing liquid samples. Journal of Chemical Education, 44(9):530, 1967.

[150] B Rosenberg and E. Postow. Semiconduction in proteins and lipids - its possible biological importance. Ann. N.Y. Acad. Sci., 158:158, 1969.

[151] Sandra J Rosenthal, Ralph Jimenez, Graham R Fleming, PV Kumar, and Mark Maroncelli. Solvation dynamics in methanol: Experimental and molecular dynamics simulation studies. Journal of Molecular Liquids, 60(1-3):25-56, 1994.

[152] C Ruckebusch, M Sliwa, P d Pernot, A De Juan, and R Tauler. Comprehensive data analysis of femtosecond transient absorption spectra: A review. Journal of Photochemistry and Photobiology C: Photochemistry Reviews, 13(1):1-27, 2012.

[153] Tadeusz Sarna and Harold A. Swartz. The Physical Properties of Melanins. 2007.

[154] Florian Scheck. Muon physics. Physics Reports, 44(4):187-248, 1978.

[155] B Schmidt, S Laimgruber, W Zinth, and P Gilch. A broadband kerr shutter for femtosecond fluorescence spectroscopy. Applied Physics B, 76(8):809-814, 2003.

[156] G. D. Scholes and G. Rumbles. Excitons in nanoscale systems. J. Phys. Chem. B, 5:683-696, 2008.

[157] Haruo Shizuka. Excited-State Proton-Transfer Reactions and Proton-Induced Quenching of Aromatic Compounds. Accounts of Chemical Research, 18(5):141-147, 1985.

[158] Ron Simkovitch, Naama Karton-Lifshin, Shay Shomer, Doron Shabat, and Dan Huppert. Ultrafast Excited-State Proton Transfer to the Solvent Occurs on a Hundred-Femtosecond TimeScale. J. Phys. Chem. A, 117:3405-3413, 2013. 
[159] Ron Simkovitch, Shay Shomer, Rinat Gepshtein, and Dan Huppert. How fast can a protontransfer reaction be beyond the solvent-control limit? The Journal of Physical Chemistry B, 119(6):2253-2262, 2015.

[160] D. V. Sivukhin. A General Course in Physics. Optics, 4 [in Russian]. Nauka, 1980.

[161] DA Skoog, DM West, FJ Holler, and SR Crouch. Fundamentals of analytical chemistry. $7 i$ th ed. USA: Brooks/Cole, Thomson Learning Inc, 2004.

[162] Andrzej L. Sobolewski and Wolfgang Domcke. Photophysics of eumelanin: Ab initio studies on the electronic spectroscopy and photochemistry of 5,6-dihydroxyindole. ChemPhysChem, 8(5):756-762, 2007.

[163] Klaus B Stark, James M Gallas, Gerry W Zajac, Joseph T Golab, Shirley Gidanian, Theresa McIntire, and Patrick J Farmer. Effect of stacking and redox state on optical absorption spectra of melanins- comparison of theoretical and experimental results. The Journal of Physical Chemistry B, 109(5):1970-1977, 2005.

[164] HB Steen. Wavelength dependence of the quantum yield of fluorescence and photoionization of indoles. The Journal of Chemical Physics, 61(10):3997-4002, 1974.

[165] K Stratton and MA Pathak. Photoenhancement of the electron spin resonance signal from melanins. Archives of biochemistry and biophysics, 123(3):477-483, 1968.

[166] G.A. Swan. Structure, chemistry and biosynthesis of the melanins. Progress in the Chemistry of organic natural products, 1974.

[167] Seiji Takeuchi, Wengeng Zhang, Kazumasa Wakamatsu, Shosuke Ito, Vincent J Hearing, Kenneth H Kraemer, and Douglas E Brash. Melanin acts as a potent UVB photosensitizer to cause an atypical mode of cell death in murine skin. Proceedings of the National Academy of Sciences of the United States of America, 101(42):15076-81, 2004.

[168] W. C. Tan, K. Koughia, Jai Singh, and S. O. Kasap. Fundamental optical properties of materials i. Optical properties of condensed matter and applications, 6:1, 2006.

[169] J. Tauc, R. Grigorovici, and A. Vancu. Optical properties and electronic structure of amorphous germanium. Physica Status Solidi, 15:627, 1966.

[170] Karen Thorn. Ultrafast Vibrational Spectroscopy of the Eumelanin pigment. PhD thesis, Open Access Victoria University of Wellington- Te Herenga Waka, 2021. 
[171] Laren M. Tolbert and Kyril M. Solntsev. Excited-state proton transfer: From constrained systems to "super" photoacids to superfast proton transfer. Accounts of Chemical Research, 35(1):19$27,2002$.

[172] M. Linh Tran, Ben J. Powell, and Paul Meredith. Chemical and structural disorder in eumelanins: A possible explanation for broadband absorbance. Biophysical Journal, 90(3):743-752, 2006.

[173] Thanh-Nga T. Tran, Joshua Schulman, and David E. Fisher. Uv and pigmentation: molecular mechanisms and social controversies. Pigment cell E melanoma research, 21(5):509-516, 2008.

[174] Andrew A.R. Watt, Jacques P. Bothma, and Paul Meredith. The supramolecular structure of melanin. Soft Matter, 5(19):3754-3760, 2009.

[175] Albert Weller. Quantitative untersuchungen der fluoreszenzumwandlung bei naphtholen. Zeitschrift für Elektrochemie, Berichte der Bunsengesellschaft für physikalische Chemie, 56(7):662-668, 1952.

[176] A.R. Wielgus and T. Sarna. Melanin in human irides of different color and age of donors. Pigment cell research, 18:454, 2005.

[177] JD Williams, Elaine L Jacobson, H Kim, M Kim, and MK Jacobson. Folate in skin cancer prevention. Water Soluble Vitamins, pages 181-197, 2012.

[178] Kent R Wilson and Vladislav V Yakovlev. Ultrafast rainbow: tunable ultrashort pulses from a solid-state kilohertz system. JOSA B, 14(2):444-448, 1997.

[179] M L Wolbarsht, A W Walsh, and G George. Melanin, a unique biological absorber. Optical Society of America, 20(13):2184-2186, 1981.

[180] Sander Woutersen, Y Mu, Gerhard Stock, and Peter Hamm. Hydrogen-bond lifetime measured by time-resolved 2d-ir spectroscopy: N-methylacetamide in methanol. Chemical Physics, 266(23):137-147, 2001.

[181] Jianhua Xu and Jay R Knutson. Ultrafast fluorescence spectroscopy via upconversion: applications to biophysics. Methods in enzymology, 450:159-183, 2008.

[182] Jianjun Yang and Guoguang Mu. Multi-dimensional observation of white-light filaments generated by femtosecond laser pulses in condensed medium. Optics express, 15(8):4943-4952, 2007.

[183] Tong Ye and John D. Simon. Comparison of the Ultrafast Absorption Dynamics of Eumelanin and Pheomelanin. The Journal of Physical Chemistry B, 107(40):11240-11244, 2003. 
[184] G.W. Zajac, J.M. Gallas, J. Cheng, M. Eisner, S.C. Moss, and A.E. Alvarado-Swaisgood. The fundamental unit of synthetic melanin: a verification by tunneling microscopy of X-ray scattering results . Biochimica et Biophysica Acta, 119:271, 1994.

[185] M Ziolek, M Lorenc, and R Naskrecki. Determination of the temporal response function in femtosecond pump-probe systems. Applied Physics B, 72(7):843-847, 2001. 